\title{
Alek Pohl
}

\section{Untersuchungen zur Wortbildung}

\section{Das Problem der Nominalisierung in der polnischen Gegenwartssprache}

Verlag Otto Sagner München · Berlin · Washington D.C.

Digitalisiert im Rahmen der Kooperation mit dem DFG-Projekt „Digi20“

der Bayerischen Staatsbibliothek, München. OCR-Bearbeitung und Erstellung des eBooks durch den Verlag Otto Sagner:

http://verlag.kubon-sagner.de

( bei Verlag Otto Sagner. Eine Verwertung oder Weitergabe der Texte und Abbildungen, insbesondere durch Vervielfältigung, ist ohne vorherige schriftliche Genehmigung des Verlages unzulässig.

«Verlag Otto Sagner» ist ein Imprint der Kubon \& Sagner GmbH. 


\section{SPECIMINA PHILOLOGIAE SLAVICAE}

Herausgegeben von

Olexa Horbatsch und Gerd Freidhof

Supplementband 7

Alek PahI

HNEERSUCHUNGEN ZUR WOREBILDUNG

Das Prahlem der Naminalisierung

in der

paInischen Gegenwartssprache

VERLAG OTTO SAGNER - MÜNCHEN

1985 


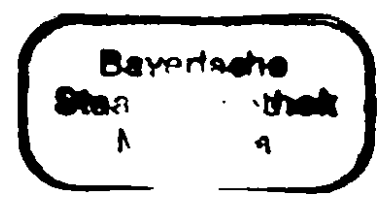

ISBN $3-87690-298-3$

Copyright by Verlag otto Sagner, München 1985 . Abteilung der Firma Kubon und Sagner, München. Druck: Gơrich \& Weiershăuser, 3550 Marburg/L. 
Das Polnische gehort in Hinblick auf die Morphologie zweifelsohne zu den interessanteren indoeuropaischen Sprachen. Unter diesem Gesichtspunkt kann es 2.B. neben das Deutsche, aber auch - mit Einschränkungen - neben das Italienische und das Spanische gesetzt werden, also neben Sprachen, die, verglichen $2 . B$. mit dem Englischen oder dem Französischen, morphologisch "ergiebiger" sind. Unter den slavischen Sprachen steht das Polnische, morphologisch gesehen, dem Tschechischen am nächsten, wenngleich es auch wie das Russische und das Serbokroatische nicht nur stark flexions-, sondern auch affigierungsorientiert ist.

Dem allgemeinen sprachtypologischen Gesichtspunkt eines flektierenden bzw. Glexivisch-analytischen Typs von Sprachen, zu dem die meisten indoeuropäischen sprachen - vgl. dazu u.a. Ineichen (1979, Kap. IV, VII) - zählen, entspricht im Polnischen ein abgeschlossenes Inventar von Flexionsendungen neben einem umfangreichen Inventar von Affixen, die bestimmte lexikalische Kategorien ausdrucken. Affixe, die lexikalische Kategorien ausdrücken, werden sowohl der Wortwurzel als auch dem, bereits affigierten Wortstamm hinzugefügt und sind von den Flexionsendungen getrennt zu sehen; denn die Flexionsendung wird erst dem endgultig affigierten Wortstamm hinzugefügt.

Das umfangreiche Inventar von Affixen gibt dem Polnischen seinen Reichtum an Derivationsmöglichkeiten, wie sie unter den nichtslavischen Sprachen 2.B. in Italienischen - wenngleich nicht in diesem AusmaB - vorzufinden sind. So entsprechen z.B. dem italienischen encomiabile 'lobenswert' die polnischen worter ahwalny und chwalebny (tschechisch chvalny, ohvalitebny; russisch pochval'nyj; serbokroatisch hvalan, pohvalnol, dem italienischen encomio 'Lob' entsprechen die polnischen worter pochwaza, chwalba (tsch. chvala, pochvala; russ. pochvala, chvala; skr. hvala, pohvala), den ital. encomiatore 'Lobender' die polnischen worter chwalca, chwalczy, dem ital. encomiastico 'Lob-' das pol. pochwalny. Obendrein verfügt das Polnische uber eine Reihe von Deverbativa, die diese Derivationsreihe fortsetzen IieBen. 
Der Affigierungsorientiertheit des Polnischen steht die Wortstammorientiertheit des Deutschen gegenüber, so z.B. in 20ben-s-wert vs. chwal-ebn-n-y/chwal-n-y. Und es kann behauptet werden, daß das Polnische grundsätzlich mit einem Wortstamm auskommt; dort, wo das Deutsche eines zweiten Wortstamms bedarf, gilt - in der Regel - im Polnischen ein entsprechendes Affix, z.B. in 2igaretten-etui vs. papieros-nic-a. Hier ist auch die genannte Einschränkung gegenüber dem Italienischen anzusetzen, denn da heibt es porta-sigarette.

Die Behauptung, daB das Polnische affixorientiert sei, schlieBt nicht aus, $d a \beta$ es im Polnischen auch Komposita oder Wortverbindungen gibt; doch sind diese zum größten Teil Lehnübersetzungen überwiegend aus dem Latein und aus dem Deutschen. Es herrscht im Polnischen die Tendenz vor, Wortverbindungen durch einstämmige Nomina zu ersetzen, was man im Deutschen nur vereinzelt vorfindet, so z.B. Dampfer statt Dampfschiff oder Ascher statt Aschenbecher. Diese Tendenz - vgl. pol. z.B. zaglowiec statt statek zaglowy 'Segelschiff' - hat das Polnische zum Teil mit anderen slavischen Sprachen gemeinsam, so z.B. mit russ. literaturka statt Literaturnaja Gazeta.

Doch im Gegensatz zum Inventar der Flektive, das im Polnischen als ein abgeschlossenes system - und dies bereits seit dem 16 . Jahrhundert - angesehen werden kann, ist das Inventar der Affixe im Polnischen - synchronisch wie diachronisch gesehen - offen. Es herrscht zwar - ebenfalls seit dem 16. Jahrhundert die Tendenz vor, u.a. bestimmte Nomina agentis auf ein einziges - im 15. Jahrhundert sind es noch drei, vier und mehr suffigierungsmöglichkeiten bei gleichbleibender Bedeutung - Suffix zu beschränken, aber dieser Prozeß ist nur in wenigen Fällen abgeschlossen.

Die Affigierungsorientiertheit des Polnischen steht - ebenso wie die Flektierungsorientiertheit - in einer engen Beziehung zu dem Teil der Phonologie, der die durch Derivation und Flexion motivierten Alternationen beschreibt. Deshalb wird zwischen solchen Alternationen, die artikulatorisch motiviert sind, und solchen Alternationen, die morphologisch, d.i. derivationell und flexionell motiviert sind, unterschieden. $\mathrm{zu}$ den artikulatorisch motivierten Alternationen zählt im Polnischen u.a. 
die Stimmlosigkeit des Obstruenten vor einem stimmlosen Obstruenten oder die Stimmlosigkeit des Obstruenten im Wortauslaut. Zu den morphologisch motivierten Alternationen zählt z.B. die Alternation der Vokale $a / e$ oder der Liquida $r / \dot{r}$ z.B. in wiar-al / wier-n-y / wierz-e 'Glaube/Glaubender, treu/(DAT, LOK SG) Glaube'.

Die morphologisch motivierten Alternationen des Polnischen betreffen also den sog. Vokal- und den sog. Konsonantenwechsel, die - im Sinne der Diachronie - bestimmten Gesetzmäßigkeiten innerhalb der Geschichte der polnischen Sprache entsprechen. Um jedoch diese Alternationen in die Affigierung - oder in die Flexion - einsetzen zu können, sind Regeln nötig, die das Funktionieren des sog. Vokal- und Konsonantenwechsels unter bestimmten morphosyntaktischen Voraussetzungen zeigen.

Zur morphologisch motivierten Phonologie des Polnischen wären hier hauptsächlich Laskowski (1975), Gussman (1978) und (1980) sowie Dunaj (1979) zu nennen.

Die Zahl der synchronischen Untersuchungen zur Wortbildung der polnischen Gegenwartssprache ist stattlich. Unter den neueren, umfassenden Arbeiten ragen besonders Fokker (1966), Brodowska-Honowska (1967), Miodek (1976), Kowalik (1977), Grzegorczykowa (1979), Grzegorczykowa \& Puzynina (1979) sowie Lubaszewski (1982) hervor.

Fokker (1966) beschränkt sich auf die deverbalen Nomina. Er listet sie nach suffixen auf und hebt bestimnte produktive Typen hervor. Das Morphem sowie der Vokal- und der Konsonantenwechsel werden am Beispiel veranschaulicht.

Brodowska-Honowska (1967) unternimmt den Versuch, die Derivation im Polnischen als ein System darzustellen, wobei neben der lexikalischen Kategorie, von der das entsprechende Nomen abgeleitet wird, Klassifizierungen der traditionellen Grammatik verwendet werden. Bemerkenswert sind die Bemühungen, eine Paraphrase des jeweiligen Derivats einzubeziehen, sowie die Verwertung der Untersuchungen zur Wortbildung im Polnischen von $w$. Doroszewski, z. Klemensiewicz u.v.a.

Miodek (1976) sammelt die zahlreichen sog. synthetischen lexikalischen Konstruktionen des Gegenwartspolnisch und legt ihnen 
jewellige Wortverbindungen bzw. Paraphrasen zugrunde, die ebenfalls 2.T. auf ältere Untersuchungen zurückgehen. Diese Arbeit stellt eine "Fundgrube" von Paraphrasen und Wortverbindungen dar, die besonders für die Untersuchung der sog. Univerbierung von Nutzen sind.

Kowalik (1977) beschränkt sich auf die Morphologie des Adjektivs und zeigt die zahlreichen Möglichkeiten von dessen Affigierung, wăhrend Grzegorczykowa (1979) die Wortbildung anhand der lexikalischen Kategorien darstellt, von denen die jeweiligen worter abgeleitet werden.

Lubaszewski (1982) analysiert das polnische Verb nach morphosyntaktischen Kriterien und versucht, die verbbildung in die Generativistik einzubeziehen.

AuBer diesen Arbeiten sei u.a. auf Honowska (1979) hingewiesen, wo allein die Untersuchungsmethoden der polnischen synchronischen wortbildung in den Jahren 1967-1977 Thema sind, sowie auf die 122 Positionen der Bibliographie in Grzegorczykowa \& Puzynina (1979), die überwiegend Einzeluntersuchungen zur Wortbildung im Polnischen enthalten.

AuBer Lubaszewski (1982) ist den genannten Arbeiten gemeinsam, das sie die Phonologie bzw. die Morphonologie entweder als gegeben voraussetzen oder als mehr oder weniger zufällige Randerscheinung der Derivation notieren oder aber auber acht lassen. So bleiben auch die genannten Untersuchungen zur Phonologie bzw. Morphonologie des Polnischen von diesen Untersuchungen zur Wortbildung des Polnischen isoliert.

Die vorliegende Untersuchung der Nominalisierung, d.1. der Bildung der Nomina in Gegenwartspolnisch stellt einen Versuch dar, diese beiden Gebiete, d.i. die Phonologie und die Wortbildung zusammenhängend darzustellen, indem u.a. der Wortbildung ein wortphonologisches Regelsystem vorausgeschickt wird, das das Funktionieren hauptsächlich der morphologisch motivierten Alternationen zeigt und somit auch in die Wortbildung einbezogen werden kann.

Den theoretischen Rahmen dieser Untersuchung bildet im weitesten Sinne die sog. Erweiterte Standard-Theorie der Generativistik, die ungeachtet der Tatsache angewandt wird, das sie - als 
Standard-Theorie - besonders zwischen Rhein und Elbe - sicherlich durch "Uberstrapazierung" besonders in den 70-er Jahren - an Beachtung verloren hat. Im Falle der Nominalisierung im Polnischen bietet sie in ihrer erweiterten Form jedoch die Möglichkeit, die Wortbildung des Polnischen in ihrer Eigenart beschreibungsadäquat darzustellen.

Innerhalb dieses theoretischen Rahmens wird u.a. auch eine Möglichkeit gezeigt, die Wortverbindung bzw. die Paraphrase eines Worts ohne die Notwendigkeit der Durchführung von syntaktischen Transformationen in die Wortbildung einzubeziehen.

Die vorliegende Untersuchung ist die überarbeitete Fassung meiner im Jahre 1982 von der Philosophischen Fakultät der Georg-August-Universität Göttingen angenommenen Habilitationsschrift. Für anregende Gespräche bin ich auBer Professor A. de Vincenz den Professoren Andrzej Bogusławski, Harald Fricke, Thomas Gardner, Olexa Horbatsch, Gustav Ineichen, Roman Laskowski, Reinhold Olesch, Olof Paulsson, Wolfgang P. Schmid, Wolf Thümmel sowie den Herren Günter Behrendt, OStR., Gerd Hentschel, M.A. und Eckhard Eggers dankbar.

Nicht zuletzt gilt meine dankbare Erinnerung meinem Lehrer Professor Dnitrij Tschižewskij sowie Professor Aleksandr V. Isačenko.

Für die finanzielle Hilfe danke ich Herrn Eugen Sonntag, Dipl. Ing.

Göttingen, im April 1984

Alek Pohl 
I-1'

if

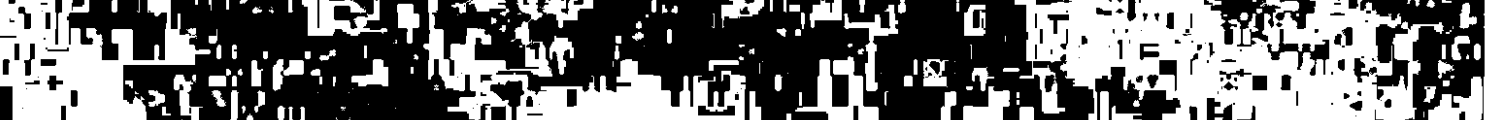

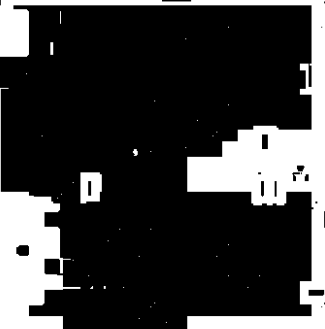

Plo

IT:

1)

T.

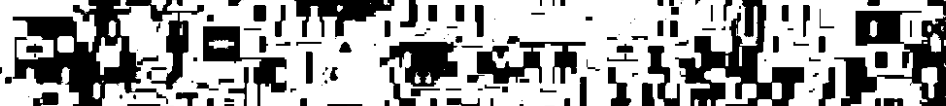

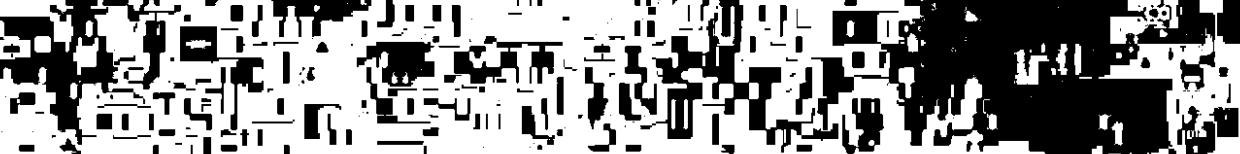

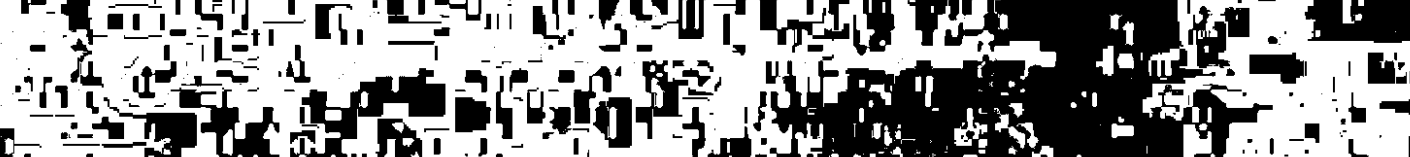
$x_{1}-x_{-1}$ Q

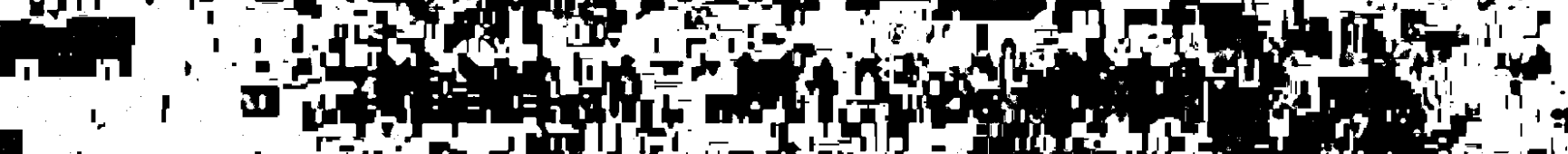

I I I I

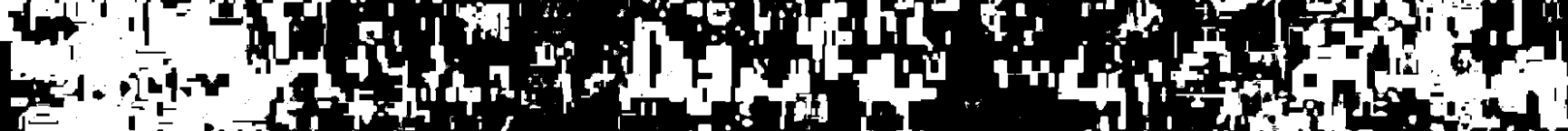

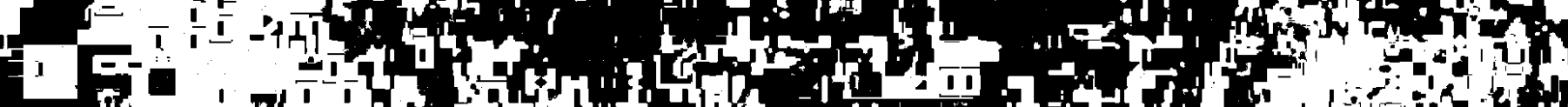

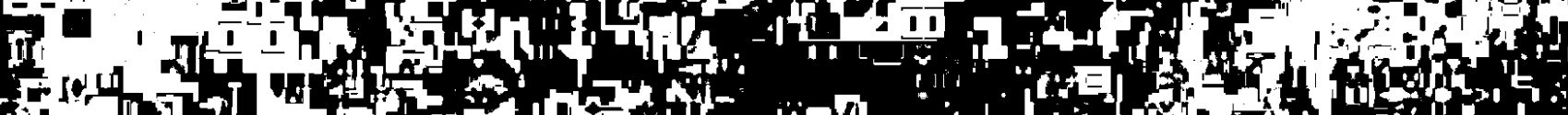

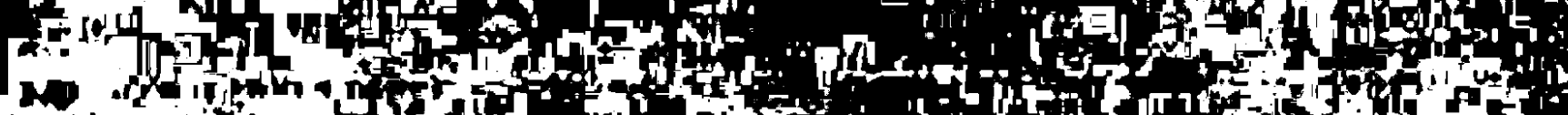

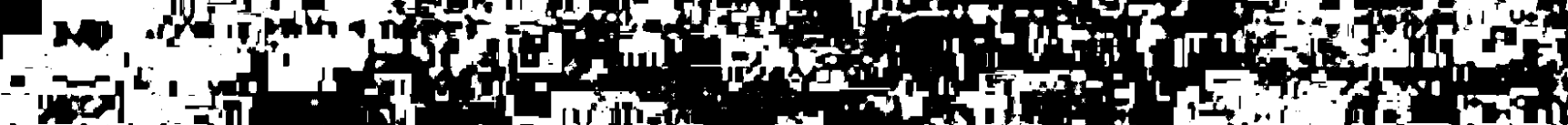
Ix),

1. $\because 5$. $T$,

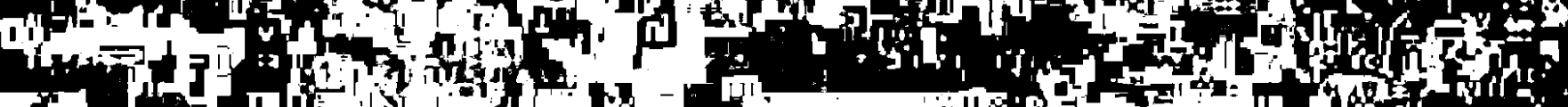

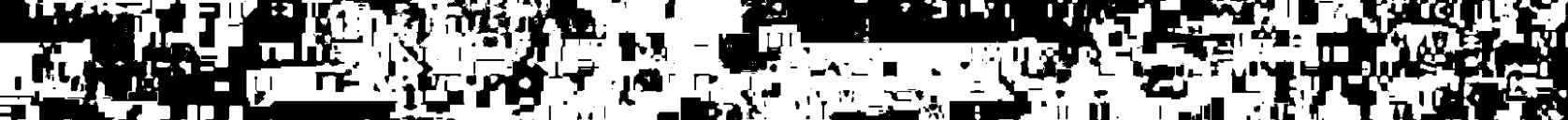
F, "

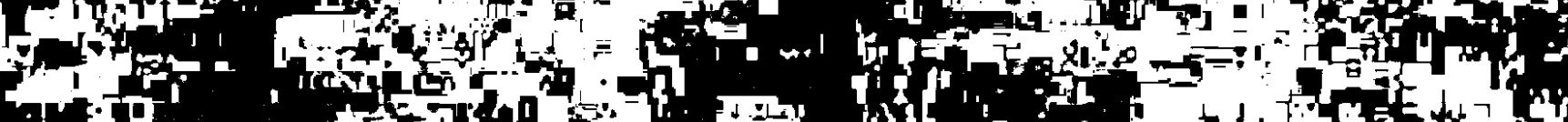

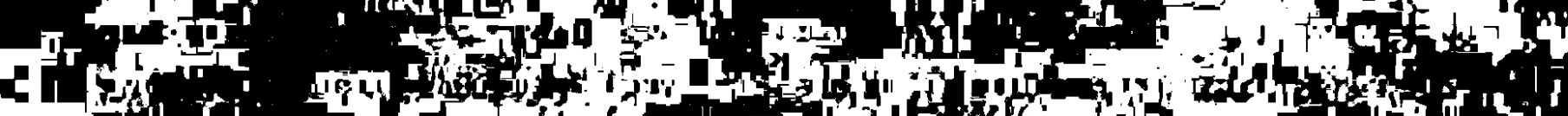

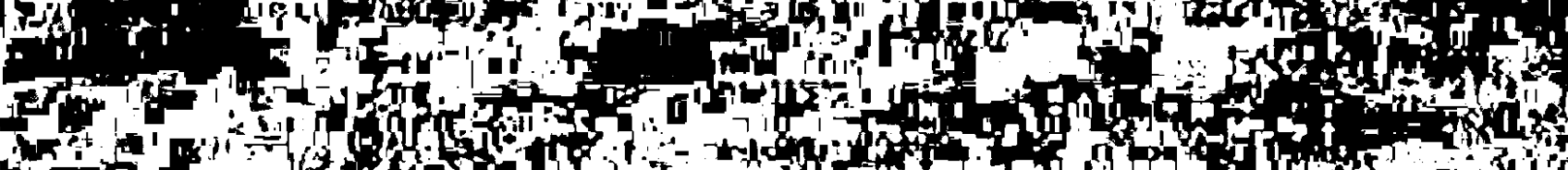

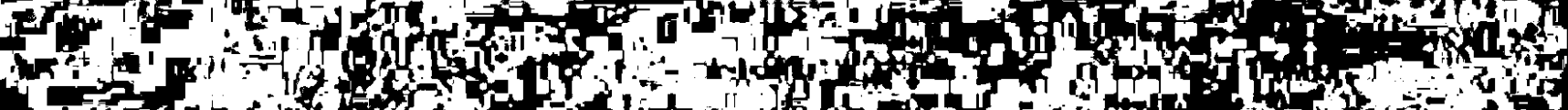

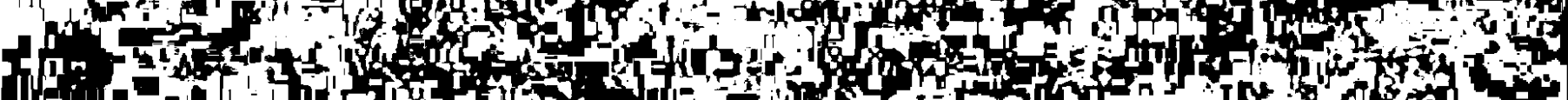

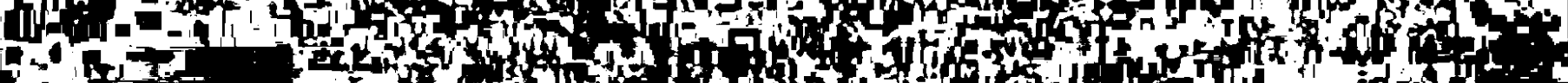

Jit

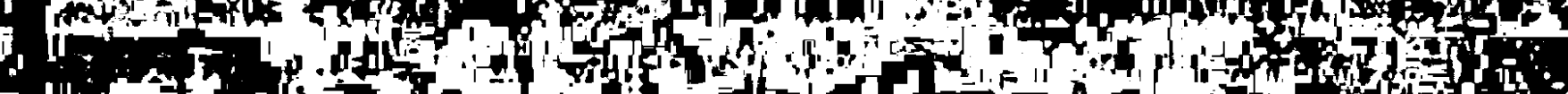

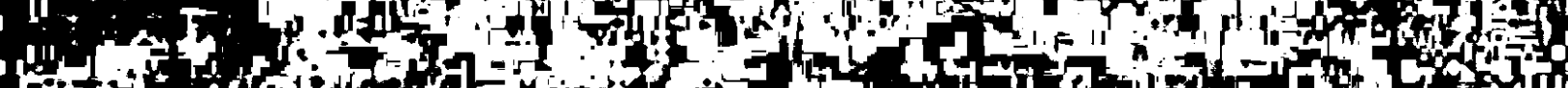

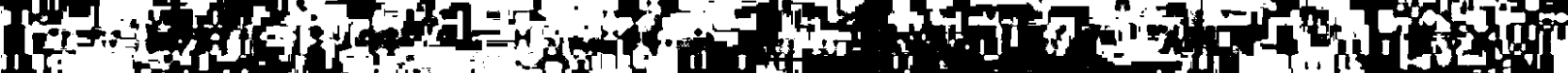

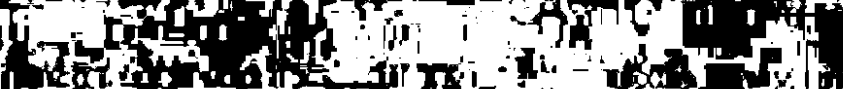

\section{.}

D.t.

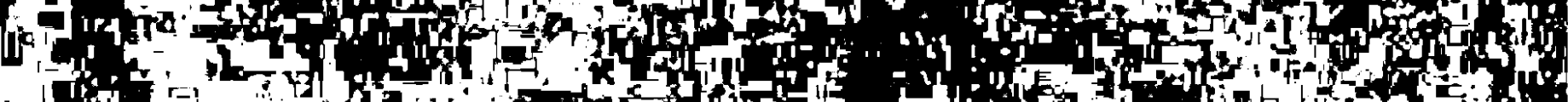

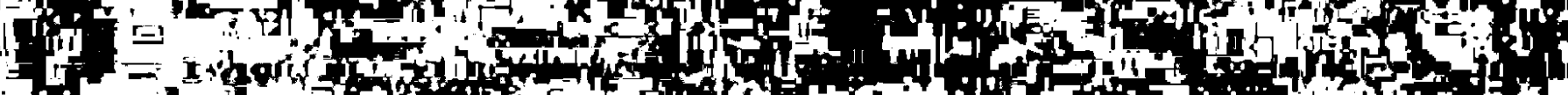

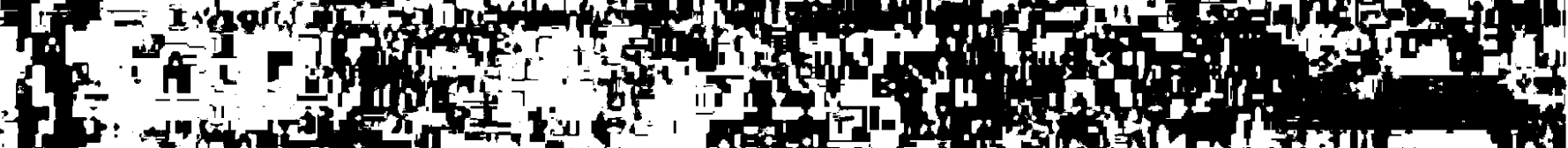

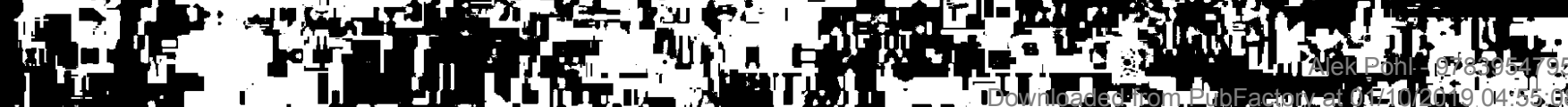
in 
INHALT

Vorwort . . . . . . . . . . . . . . 5

Symbole und Abkürzungen . . . . . . . . . . 13

1.0 Das Wort . . . . . . . . . . . . . . . 15

1.1 Behauptungen . . . . . . . . . . . . 19

2.0 Wortphonologie .............. 21

2.1 Markiertheit und Binarität . . . . . . . . 21

2.2 Merkmale . . . . . . . . . . . . . . 23

2.3 Alternationen . . . . . . . . . . . . . 24

2.4 Wortgrenzen ................... 27

3.0 Morphologie . . . . . . . . . . . . . 29

3.1 Syntaktische und morphologische Komponente . . 29

3.1.1 Generalisierter Wortmarker . . . . . . . . 31

3.1.2 Inhärente Merkmale . . . . . . . . . . . 32

4.0 Wortsemantik . . . . . . . . . . . . . 35

4.1 Semantische Komponente . . . . . . . . . . 35

4.2 Teilauswertung ............... 43

5.0 Folgen für die Wortbildung . . . . . . . . . 47

5.1 Chomsky 1970 . . . . . . . . . . . . . . 47

5.2 Halle, Jackendoff, Aronoff . . . . . . . . 51

5.3 Laskowski 1981 . . . . . . . . . . . . . 54

5.4 Teillauswertung . . . . . . . . . . . . 58

5.4.1 Partizip und deverbales Nomen . . . . . . . . 58

5.4 .2 Univerbierung . . . . . . . . . . . . . 65

6.0 Die morphosyntaktische struktur . . . . . . . 71

6.1 Der Wortbeugungsteil . . . . . . . . . . 76

6.2 Der Wortbildungsteil . . . . . . . . . . 76

6.2.1 Die Morphemmarker . . . . . . . . . . . . 77

6.2.1.1 Die Wurzel (R) . . . . . . . . . . . . . . 77

6.2.1.2 Das Stammformativ (TF) . . . . . . . . . . 78

6.2.1.3 Klassifikator (K) und Stamm-Marker (TM) . . . . 78

6.2.1.4 Das Prăfix (PF) . . . . . . . . . . . . . 79

6.2 .1 .5 Das Infix (IF) . . . . . . . . . . . . . 79

6.2 .1 .6 Der Evaluator (EV) . . . . . . . . . . . 80

6.3 Die morphosyntaktische struktur (61) . . . . . 80

7.0 Wortbildung ................. 81 
7.1 Morphonologie des Polnischen . . . . . . . . 81

7.1.1 Merkmalphonologisches System des Polnischen - 82

7.1.1.1 Die Oberklassenmerkmale . . . . . . . . . . 83

7.1.1.2 Die Liquida . . . . . . . . . . . . . . . 84

7.1.1.3 Das Morphoneminventar des Polnischen (68) . . 85

7.1.2 Die morphonologischen Regeln . . . . . . . . . 87

7.1.3 Die morphonologischen Tilgungsregeln . . . . . . 96

7.1.3.1 Der morphonologische operator Q . . . . . . . . 97

7.2 Merkmale . . . . . . . . . . . . 99

7.2.1 Lexikalische Kategorie . . . . . . . . . 100

7.2.2 Genus und Geschlecht . . . . . . . . . . . 102

7.2.3 Aspekt und Genus Verbi . . . . . . . . . . . 104

7.2.4 Die inhărenten Merkmale . . . . . . . . . 105

7.3 Die Wortbildungsregeln . . . . . . . . . 108

8.0 Nominalisierung im Polnischen . . . . . . . . 113

8.1 Wurzelnomina . . . . . . . . . . . . . . . 114

8.2 Denominale Nomina . . . . . . . . . . . 119

8.3 Deadjektivische Nomina . . . . . . . . . . . 142

8.4 Deverbale Nomina . . . . . . . ...... 151

8.4.1 Die sogenannten Verbalwörter . . . . . . . . 162

9.0 Bestandsaufnahme und Auswertung . . . . . . 178

9.1 Zur Einbeziehung der Wortverbindungen . . . . 178

9.2 zur morphosyntaktischen struktur . . . . . . . 183

9.3 Zur Affigierung . . . . . . . . . . . . 185

9.4 schlub . . . . . . . . . . . . . . 189

Anmerkungen . . . . . . . . . . . . . . 192

Literatur . . . . . . . . . . . . . . 204

Sumnary . . . . . . . . . . . . . . . 210

Streszczenie................. 213 
SYMBOLE UND ABKURZUNGEN

( ) Fakultativ-Klammer

/ / Phonem-Klammer

[ ] Merkmal, Symbol komplexer Merkmale, Phon (Laut)

[+ +] Morphem, Morphonem innerhalb der Wortgrenzen

\{ \} Morphem, Morphonem, Alternation

= vordere Wortgrenze

- vordere Wurzelgrenze, hintere Präfixgrenze

+ hintere Wurzelgrenze, TM-, TF-, IF-, K-Grenze

\# hintere Wortgrenze, hintere FL-Grenze

$\rightarrow$ "wird ersetzt"

$\rightarrow \quad$ "wird zugeordnet"

$\rightarrow$ / "wird ersetzt, wenn"

- / "wird zugeordnet, wenn"

C Konsonant, Gleitlaut

V Vokal

* Konsonantenwechsel (entspr. der 1. Palatalisierung)

* vor Wort: hypothetisch

- Konsonantenwechsel: alle anderen Palatalisierungen

*/, "entweder Konsonantenwechsel * oder Konsonantenwechsel ,"

* "sowohl Konsonantenwechsel * als auch ,"

$X$ _ $Y$ "in der Ungebung von $X$ und $Y$ "

8 Vokal-Null-Alternation

$\&_{1} \quad \tilde{e} / \tilde{o}$ - Alternation

\& 2 e/a - Alternation

\& $u / 0$ - Alternation

\& 4 u/e/a/o - Alternation

\& $\quad$ u/o/i - Alternation

(PR) phonologische Redundanzregel

(PT) phonetisch motivierte Regel

(PL) morphonologisch motivierte Regel

(DL) morphonologische Tilgungsregel

(DER) Wortbildungsregel

(AF) Affigierungsregel

$\mathrm{N} \quad$ Nomen

V Verb

A Adjektiv

P Präposition

ADV Adverb 
PRO PRONOMEN, PRO-Element

$t_{i} \quad$ Trace (Spur)

S Satz

W Wort

LFT lexikalischer Formationsteil

GFT grammatischer Formationsteil

R Wortwurzel

$T$ Wortstamm

TM Stamm-Marker

PF Präfix

IF Infix

TF Stamm-Formativ

K Klassifikator

EV Evaluator

FT Flexionsteil

FL Flektiv

WB Wortbildung

NOM Nominativ

GEN Genitiv

DAT Dativ

AKK Akkusativ

INS Instrumental

LOK Lokativ

SG Singular

PL Plural

DOR W. Doroszewski /Red./, Słownik języka polskiego. Warszzwa 1958 - 1969

IAT Indeks a tergo do Słownika jezyka polskiego pod redakcja Witolda Doroszewskiego. Warszawa 1973

KAR M. Karas /Red./. Słownik wymowy polskiej. Kraków 1977

LIN M.S.B. Linde, Słownik jezyka polskiego. Lwow 1954 - $18 j 0$

SkA F. Sławski, Słownik etymologiczny jezyka polskiego. Kraków 1952 -

SPV Słownik polszczyzny XVI wieku. Wrocław et al. 1966 -

STP Słownik staropolski. Warszawa 1953 -

SW Słownik języka polskiego ułożony pod redakcją J. Karłovicza, A. Kryńskiego i W. Niedzwieckiego /"Słownik Warszawski"/. Warszawa $1900-1927$ 


\subsection{DAS WORT}

Das Wort ist Element der Sprache. Als solches ist es auch neben anderen Elementen der Sprache Gegenstand der Sprachwissenschaft. Ein Definition des sprachwissenschaftlichen Begriffs wort muB innerhalb einer gültigen Theorie geschehen. Und so fassen auch verschiedene sprachwissenschaftliche Schulen das wort unterschiedlich auf.

Bei Ferdinand de Saussure entspricht das Wort dem sprachlichen Zeichen (signe linguistique); dieses setzt sich aus der materiellen Form, dem Ausdruck (signifié), und dem Inhalt (signifiant) als Einheit zusammen. 'Bei Nikolaj Trubeckoj entspricht die Relation zwischen Ausdruck und Inhalt der Relation zwischen der abstrakten langue ${ }^{2}$ Saussures, im Sinne einer Invarianten, und deren Materialisierung, der parole; dies im Minimalpaar, das sich aus zwei phonetisch repräsentierten wörtern zusammensetzt und die Ermittlung distinktiver Merkmale ermöglicht. Die distinktiven Merkmale bestimmen das Phonem in seiner Funktion als kleinste bedeutungsunterscheidende Einheit der sprache; das Phonem ist invariant und gehört einem system an, das u.a. eine Sprache von einer anderen Sprache unterscheidet. Damit ist jedoch die Möglichkeit einer Begrenzung der phonetischen oder phonemischen Einheit wort nicht gegeben. ${ }^{3}$

Louis Hjelmslev nimmt in seiner Sprachtheorie " eine Hierarchie von Systemen und Verläufen an. Die Hierarchie von Systemen ist u.a. der Formenlehre, die Hierarchie von Verläufen dagegen der Syntax zugeordnet. Diese beiden Hierarchien werden Klassen von Klassen mit implizierten Derivaten gleichgesetzt, und es wird der Grad bestimmt, durch welchen die Derivate von ihrer niedrigsten gemeinsamen Klasse abhängig sind. Das Wort wird als Element des Textes gesehen, wobei die Periode Derivat ersten Grades des Textes, der Satz Derivat zweiten Grades des Textes und das Wort Derivat dritten Grades des Textes sind.

Da Hjelmslevs Derivat der Relation zwischen Ausdruck lexpression) und Inhalt (content) sowie der jeweiligen Unterteilung in Form und substanz unterliegt, kann auch das wort innerhalb dieser zuordnungen gesehen werden, z.B. lautlich oder graphemisch unter dem Gesichtspunkt der Ausdruckssubstanz, bezüglich seines 
Stamms und der ihm zugefügten oder nichtzugefügten Affixe unter dem Gesichtspunkt der Ausdrucksform und bezüglich seiner Bedeutung unter dem Gesichtspunkt der Ausdrucksform und der Ausdruckssubstanz. Damit wären einige Relationen genannt, die es erlauben, das Wort aufgrund seiner struktur zu bestimmen, vorausgesetzt man berücksichtigt seine Abhängigkeit von der niedrigsten gemeinsamen Klasse von Derivaten bzw. Komponenten; in diesem Fall wäre es der Satz. Denn der Satz entspricht bei Hjelmslev der Komponente ersten Grades der Periode, das Wort der Komponente ersten Grades des Satzes und zweiten Grades der Periode. So gesehen entspricht das wort einer GröBe - vgl. Hjelmslev (1953, $\S 11)$-, die als Voraussetzung fúr andere Gróssen, wie u.a. Satz, Periode gesehen wird. s

So kann das wort einerseits bezüglich seiner syntaktischen Funktion, anderseits bezüglich seiner struktur bestimmt werden, die wiederum nicht nur der Relation zwischen Ausdruck und Inhalt, sondern zusätzlich der jeweiligen Unterscheidung zwischen Form und Substanz unterliegt.

Der Strukturalismus hat das Morphem als kleinste bedeutungstragende Einheit der Sprache verstanden; dies in Anlehnung an die Methode Trubeckojs. Demnach ist das Morphem die Invariante der Varianten Morph, ebenso wie das Phonem die Invariante der Varianten Phon ist. Wird das Phonem anhand von Phonen ermittelt, so kann das Morphem anhand von Morphen ermittelt werden, wobei jedoch das Phonem als Element des Morphems vorausgesetzt wird.6 Wird das Morphem in das Hjelmslevsche Sprachmodell eingesetzt, so kann festgestellt werden, daB die phonemischen Repräsentationen des Morphems einerseits als Korrelate, d.i. als Glieder. des Systems, anderseits als Relate, d.i. als Teile des Verlaufs gesehen werden können. Dabei ist hinzuzufügen, daß der Verlauf das System determiniert. So gesehen entspricht das wort, definiert als Derivat, syntagmatisch der logischen Konjunktion von Morphemen - unter Einbeziehung des sog. Null-Morphems ${ }^{7}$ - paradigmatisch dagegen der logischen Disjunktion von Morphemen.

Bei der Abgrenzung des Worts von anderen Elementen der Sprache fällt auch seine Zugehörigkeit zum Lexikon ins Gewicht; der Begriff Wort wird durch den Terminus Lexem ersetzt, das u.a. als ein autonomes Syntagma von Morphemen aufgefaßt und in das Lexi- 
kon eingereiht wird. ${ }^{8}$ Hier wird das Wort, wie es im Lexikon steht, von seiner flektierten Form im Text abgegrenzt. Der Begriff Wort wird also einerseits als Einhelt des Lexikons, anderseits als Element des Textes gesehen.

Bereits im Jahre 1888 ordnet Jan Baudouin de Courtenay die individuelle Sprache dem Individuum und dessen Sprachkompetenz (uzdolnienie jezykowel zu. Er unterteilt die individuelle sprache in psychisch-physische und in akustisch-physiologische bzw. phonetisch-akustische Elemente, zwischen denen Assoziation besteht.9 Das Wort gehört zu den psychisch-sprachlichen Elementen und wird durch Assoziation dem Lautunterschied Iróznica diwieków) zugeordnet. Das Morphem wird von Baudouin de Courtenay bereits 1895 dem Lautgewand (szata gzosowa), der phonemische 1 lternant der Alternation und das Phonem als psychisches Aquivalent der sprachlaute dem Laut zugeordnet. Die psychischen Sprachelemente, darunter auch das wort, entsprechen bel Baudouin de Courtenay der inneren Seite der Sprachkompetenz, die dem Sprecher, der des Kulturpolnischen (polszczyzna kulturalna) măchtig 1st, zugeordnet wird, wahrend die physiologisch-phonetischen Sprachelemente der äuBeren Seite der Sprachkompetenz entsprechen.

Baudouin de Courtenay stützt sich ebenfalls auf das Morphem, auf dessen Eigenschaft als einfachstes Element, als psychisch-sprachliches Element im Lautgewand sowie auf dessen Verknupfung mit anderen Morphemen. Die Verknüpfung der Morpheme untereinander geschieht mittels eines Exponenten (wykzadnik), der jedem Morphem eigen ist; solch ein Exponent gilt auch fur die Verknüpfung der wörter in Satz $(1888,38)$.

Die Festlegung des Morphems ist bei Baudouin de Courtenay mit der Annahme von phonetischen Alternationen verbunden, die als Element des Morphems gesehen werden und damit der Bedeutung assoziativ zugeordnet sind. Aufgrund der Klassifizierung des Phonems als psychisches ãquivalent der Sprachlaute $(1895,271)$ sowie der Alternationen, die historisch motiviert sind, als korrelierende Alternanten hat das Phonem nicht nur lautliche Differenzierungsfunktion; es dient ebenso als Mittel zur Morphologisierung sowohl der phonetischen als auch der semasiologischen vorstellungen $(1908,170)$. 
Die Generativistik unterteilt die Sprache, die dem idealisierten Sprecher-Hörer zugeordnet ist, in Meaning und Sound. Meaning wird durch die Intrinsic Semantic Interpretation und Sound durch die ldeal Phonetic Form (Chomsky \& Halle 1968,3f.) bestimmt. Zwischen Sound und Meaning besteht Assoziation. Die Kcnntnis der Grammatik bestimmt die Sprachkompetenz des Sprecher-Hörers, deren Aktualisierung die Performanz darstellt.

Die generative Phonologie benutzt den Sound, um u.a. das Phonem durch akustisch-artikulatorische Merkmale darzustellen. Da dies nicht losgelöst von der Umgebung geschehen kann, wird zwischen Segment und Grenze unterschieden. Morris Halle $(1959,19)$ schreibt dazu:

(1) In phonology, speech events are represented as sequences of entities of two kinds: segments, to which specific phonetic (articulatory as well as acoustical) properties are assigned, and boundarics, which are characterized solely by their effects on the former.

Das Morphem wird von Halle $(1959,57)$ aufgrund von occurence of distinctive feature complexes in the sequence ermittelt und anhand des Russischen in Morpheme Structure Rules gefabt. Damit wird das Morphem als ein Symbol von phonetisch bedingten distinktiven Merkmalen innerhalb seiner Grenzen dargestellt, die aufgrund der sprachspezifischen Umgebung festgesetzt werden.

Um das Wort mittels der phonologie des Morphems, also mittels der Morphonologie - sowohl vom Standpunkt der Analyse als auch vom Standpunkt der Erzeugung - zu beschreiben, unterscheidet Halle (1959,48ff.) zwischen folgenden Grenzen: Phonemic Phrase Boundaries, word Boundaries, Prefix and Preposition Boundaries und suffix Boundaries.

Die einander assoziativ zugeordneten Sound und Meaning, die die Sprachkompetenz des idealisierten Sprecher-Hörers voraussetzen, sind die Grundlegungen für Chomsky \& Halle (1968), die auf den Terminus Morphem verzichten und die Morphem Structure Rules von Halle (1959) durch lexikalische Redundanzregeln llexical redundancy rules) $(1968,171)$ ersetzen. Die Formativgrenze wird beibehalten. 
In diesem Rahmen wird das Wort von Chomsky \& Halle $(1968,367)$ folgendermaßen definiert:

(2) Let us next define the "terminus" of a word as being any configuration of boundaries and brackets having the form (117) (where $S$ is the category "sentence" and $x$ contains no segments):

$$
\begin{array}{ccc}
\text { s } & x & {[\#} \\
\#] & x & \#] s \\
\#] & x & {[\#}
\end{array}
$$

Suppose that we have a string ... $Y \ldots=\ldots z$ [W\#] $V$, where $Z$ [\#and\#] $V$ are termini as defined in (117), and $Y$ contains no other termini. Then [\# $W$ ] is a word.

Diese Definition des Worts berücksichtigt sowohl die Funktion des Worts im Satz (siehe: Chomsky \&alle 1968,368) als auch die äuBeren und die inneren Wortgrenzen (siehe: \$2.4). Die einzelsprachliche Aktualisierung des so definierten worts geschieht mittels Regeln, die die Einsetzung von entsprechenden distinktiven Merkmalen ermöglichen. Diese Merkmale stehen für Morphoneme, während der Terminus Morphem durch Formativ ersetzt wird.

Die Formative werden in lexikalische und grammatische unterteilt (Chomsky \& Halle 1968,9). Uber das lexikalische Formativ schreibt Chomsky $(1965,81)$ :

(3) Each lexical formative is represented as a sequence of segments, each segment being a set of features. In other words, each lexical formative is represented by a distinctive feature matrix in which the columns stand for successive segments, and the rows for particular features.

Und Chomsky \& Halle $(1968,9)$ beziehen die Merkmal-Matrix auch auf die grammatischen Formative.

\subsection{BEHAUPTUNGEN}

Die Generativistik sieht das wort von zwei seiten: einerseits von seiner stellung im Satz, anderseits von seiner morphonemischen Struktur her. Seine Stellung im Satz ist u.a. dadurch ge- 
kennzeichnet, daB es in die Basis-struktur (D-Structure) eingesetzt wird und daB es gegen andere worter austauschbar ist. Voraussetzung für die Einsetzung des Worts in den Satz ist sein Vorhandensein im Lexikon sowie seine morphonemische struktur. Anstelle einer Definition des Begriffs Wort werden hier im Hinblick auf den Gegenstand der folgenden Untersuchung Behauptungen aufgestellt, die im Rahmen der Generativistik bleiben. Innerhalb dieses Rahmens wird das wort ebenso als Einheit des Lexikons wie als Element des Satzes bzw. des Textes gesehen, wenngleich der Schwerpunkt auf seiner morphonemischen struktur liegt, die Grundlage für die Wortbildung ist.

Es gelten folgende Behauptungen:

(4) (a)das Wort ist Einheit des Lexikons, das alle existierenden wörter einer Sprache enthält;

(b) das Wort entspricht einer Gruppe - im Sinne von geordnete Menge - von Segmenten, die Morphemen bzw. Morphemgruppen entsprechen;

(c) das Morphem ist die kleinste Einheit der Sprache, der eine bestimmte, bedingt selbständige Bedeutung zugeordnet werden kann;

(d) das Morphem setzt sich aus Segmenten zusammen, die Morphonemen entsprechen, welche sich ihrerseits aus einzelsprachlich bedingten, phonetisch motivierten Merkmalen zusammensetzen;

(e)das Morphem setzt Grenzen voraus; deshalb wird zwischen inneren und äuBeren Grenzen des Worts unterschieden; dabei kann die vordere Grenze des Morphems zugleich die vordere Grenze des Worts und die hintere Grenze des Morphems zugleich die hintere Grenze des Worts sein;

(f)die äuBeren Grenzen des Worts bestimmen Wortanfang und Wortende, die inneren Grenzen des Worts bestimmen das Formativ;

(g)ein Formativ ist ein innerhalb der äuBeren Wortgrenzen in Funktion gesetztes Morphem;

(h) das Wort wird durch die Kategorien-Merkmale $N, V, P, A D V$, PRO,... gekennzeichnet; 


\subsection{WORTPHONOLOGIE}

Gegenstand der Wortphonologie ist das Phonem als Segment des Morphems, also das Morphonem. Das Morphonem ist ein abstraktes Konstrukt, wenngleich es anhand phonetisch bedingter Merkmale bestimmt wird. Die phonetisch bedingten Merkmale korrelieren und koexistieren miteinander. Korrelation und Koexistenz der phonetisch bedingten Merkmale beruhen auf dem prinzip der Markiertheit vs. Unmarkiertheit. Die phonetisch bedingten Merkmale werden durch Oberklassenmerkmale dominiert. Die Merkmale sind artikulatorisch motiviert und unterliegen der Binarität. Aufgabe der Wortphonologie ist:

(5) (a) Bedingungen für ein Morphoneminventar des Polnischen zu schaffen, das Systemcharakter hat und auf die Hypothese eines idealisierten Sprecher-Horers gestützt ist;

(b) Bedingungen für Regeln zu schaffen, die die Redundanzen des Morphoneminventars, die morphologisch motivierten Alternationen sowie die phonetisch motivierten Alternationen erfassen;

(c) den Rahmen für die Grenzen der Morpheme im wort zu bestimmen;

(d)die Möglichkeit zu schaffen, aufgrund der unter $(4(b)-(g))$ aufgestellten Behauptungen eine morphonemische Struktur annehmen zu können, von der aus sowohl die Wortbildung als auch die Wortbeugung ihren Ausgang nehmen und von der auch die phonetische - und die graphemische - Repräsentation des Worts ableitbar sind.

\subsection{MARKIERTHEIT UND BINARITÄT}

Elemente einer Sprache, die in einer privativen Opposition $z u$ einander stehen, d.h. Elemente eines bestimmten Systems, in dem das eine Element eine bestimmte Eigenschaft enthält, die dem anderen Element fehlt, werden von Jakobson (1932) auf der Ebene der grammatischen Kategorie anhand binärer Merkmale als merkmalhaft vs. merkmallos klassifiziert. Die Relation zwischen Merkmalhaftigkeit und Merkmallosigkeit entspricht der Relation zwischen Markiertheit und Unmarkiertheit. Demnach kann ein Element gegen- 
über einem anderen aufgrund seiner Merkmalhaftigkeit markiert, aber auch unmarkiert sein; ebenso kann ein Element gegenüber einem anderen aufgrund seiner Merkmallosigkeit markiert, aber auch unmarkiert sein. ${ }^{10}$ Als graphische Darstellung gilt:

\section{MERKMALHAFT \\ MERKMALLOS}

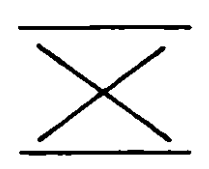

MARKIERT

\section{UNMARKIERT}

Auf der phonemischen Ebene - vgl. dazu und zum folgenden (68) - sind z.B. die Nasalvokale /ẽ/ und /õ/gegenüber /e/ und /o/ bezüglich [+ NASAL] markiert, während /e/ und /o/ bezüglich [- NASAL] unmarkiert sind. Wird z.B. die phonetisch motivierte Möglichkeit der Nasalierung des Vokals /a/ - z.B. in [šãsa] - szansa 'Gelegenheit' - berücksichtigt, so ist/a/ bezüglich [- NASAL] markiert; das gleiche gilt für die hohen vokale im polnischen, wenn sie vor Nasalkonsonanten stehen.

Die konsonantischen Sonoranten sind gegenüber den konsonantischen obstruenten bezüglich [- OBSTRUENTISCH] markiert, bezüglich [+ KONSONANTISCH] dagegen - ebenfalls gegenüber den obstruenten - unmarkiert. Markiert sind die konsonantischen Sonoranten bezüglich [+ KONSONANTISCH] gegenüber den Vokalen; desgleichen bezüglich [-SILBISCH]. Der Gleitlaut / $j /$ ist ebenfalls gegenüber den Vokalen bezüglich [- SILBISCH] markiert, gegenüber den konsonantischen Sonoranten ist er bezüglich [ - KONSONANTISCH] markiert, bezüglich [- OBSTRUENTISCH] dagegen unmarkiert. "

Werden Binarität und Markiertheit auf die phonetisch motivierten Alternationen bezogen, so läBt sich u.a. feststellen, daB bei der Alternation [ \pm STIMMHAFT] die stimmlosen Obstruenten unmarkiert, die stimmhaften dagegen markiert sind. Es wird nämlich allgemein davon ausgegangen, daB das unmarkierte Element haufiger verwendet wird als das markierte. Im Falle der opposition [ \pm STIMMHAFT] ist demnach die Stimmlosigkeit häufiger als die Stimmhaftigkeit; diese Feststellung gilt fürs Polnische nicht nur im Sinne der sog. regressiven Assimilation - 2.B. in [bap'ća] - babcia 'Oma' - sondern auch im sinne der sog. progressiven Assimilation, z.B. in [kf'at] - kwiat 'Blume' oder in [pśt] - przy 'bei'. Hinzu kommt die sog. Auslautverhärtung 
bezogen im Polnischen allein auf das Wortende. ${ }^{12}$

\subsection{MERKMALE}

Die wortphonologischen bzw. morphonologischen Merkmale entsprechen Eigenschaften, die für die Funktion des jeweiligen Segments sowohl innerhalb des Morphoneminventars als auch innerhalb des Morphems wesentlich sind. Sie sind die Voraussetzung für das Aufstellen von Strukturbedingungen, welche wiederum die Voraussetzung für das Aufstellen von Regeln sind, vgl. (5(a), (b)). Die morphonologischen Merkmale werden nach Chomsky \& Halle (1968) unterteilt - vgl. dazu (65) - in:

(7) Oberklassenmerkmale

Resonanzmerkmale

Artikulationsmerkmale

Quellenmerkmale

prosodische Merkmale

Die Oberklassenmerkmale betreffen sowohl die Funktion der Silbenbildung als auch die Eigenschaften als Geräuschlaut oder/und als Mitlaut. Die Resonanzmerkmale betreffen u.a. den Resonanzraum Mundhöhle nebst Artikulationsorgan und Artikulationsstelle. Die Artikulationsmerkmale betreffen die Artikulationsart, die Quellenmerkmale die Artikulationsquelle, während die prosodischen Merkmale u.a. den Wortakzent betreffen.

Der Wortakzent im Polnischen bewirkt eine Artikulation des Vokals, die sich von der Artikulation des nichtakzentuierten Vokals durch eine etwas längere Dauer und durch eine geringfügig stärkere Intensität unterscheidet. Diese beiden Eigenschaften führen jedoch weder zu einem distinktiven Quantitäts- noch Qualitätsunterschied; deshalb wird den prosodischen Merkmalen innerhalb der Wortphonologie keine weitere Beachtung geschenkt. Aufgrund der Binarität und der Markiertheit werden die in (7) klassifizierten Merkmale zu Bündeln zusammengefaßt; auf diese Weise bilden sie einerseits die Artikulationsbasis, anderseits dagegen die Bedingung für die Bedeutungsunterscheidung. In dieser doppelten Funktion dienen sie zur Bestimmung der Alternationen, sowohl der phonetisch motivierten (= Assimilationen) als auch der morphologisch motivierten (= Korrelationen). Denn 
sowohl auf der phonetischen als auch auf der morphonemischen Ebene alternieren niemals Konsonant mit Vokal, sondern ausschlieblich Konsonant mit Konsonant oder Vokal mit Vokal. Konsonant oder Vokal alternieren jeweils bezulglich einzelner markierter oder unmarkierter Merkmale. So alternieren auf der phonetischen Ebene z.B. [v] vs. [f] in [barva] vs. [barf] - barwa/ /barw '(NOM SG/GEN PL) Farbe' bezüglich [ \pm STIMMHAFT]. Auf der morphonemischen Ebene alternieren z.B. die Vokale \{a\} vs. \{e\} in \{m'ara\} vs. \{m'eřić\} - miara/mierzý 'MaB/messen' bezüglich u.a. [ \pm NIEDRIG], die Konsonanten $\{r\}$ vs. $\{\check{r}\}$ dagegen bezulglich [ \pm VIBRATOR]; vgl. dazu $\S 7.1 .1 .2$.

\subsection{ALTERNATIONEN}

Die Alternationen werden - wie bereits in $\S 2.1$ und $\S 2.2$ angedeutet - ihrer Motivation entsprechend unterteilt in:

(8) - phonetisch motivierte Alternationen;

- morphologisch motivierte Alternationen.

Die phonetisch motivierten Alternationen entsprechen einer $D i$ vergenz bezüglich einer bestimnten artikulatorischen Eigenschaft, wie z.B. bezüglich der stimmlosigkeit oder stimmhaftigkeit in einer bestimmten lautlichen Umgebung. Die morphologisch motivierten Alternationen entsprechen einer Korrelation bezüglich einer bestimmten artikulatorischen Eigenschaft, wie z.B. bezüglich der niedrigen Zungenlage oder der nichtniedrigen, $d$. i. der mittleren Zungenlage im Falle der Alternation der Vokale \{a\} vs. \{e\} in einer morphologisch veränderten Umgebung. Im Falle der Alternation der dentalen obstruenten \{s\} vs. \{́s\} be$z$ ulglich [ \pm PALATAL] handelt es sich dann um divergierende Alternanten, wenn die palatalität von ['s] durch die palatalität des dahinterstehenden Konsonanten bedingt ist, wie z.B. in [şfíc] - śnić 'träumen' aber [sen] - esn 'Traum, Schlaf'; ist die palatalität von \{'s\} morphologisch bedingt, wie z.B. in \{kvásni\} - kwaśny 'sauer' aber \{kvas\} - kwas 'Säure', so handelt es sich un zwei korrelierende Alternanten.

Die phonetisch motivierten Alternationen betreffen im Polnischen sowohl Vokale als auch Konsonanten. Die Vokale divergieren in der Umgebung von palatalen oder/und nasalen Konsonanten. 
In der palatalen konsonantischen Umgebung werden die Vokale weiter vorn artikuliert, jedoch in ihrer qualität nicht verändert, d.h., daB z.B. ein zwischen zwei palatalen Konsonanten artikuliertes [a] trotz seiner Verlagerung nach vorn ein niedriger, hinterer Vokal bleibt, z.B. in [ $\mathrm{K}^{\circ} \mathrm{a}^{\circ} \mathrm{ha}$ ] - niania 'Amme'. Vor nasalen Konsonanten sind die Vokale leicht nasaliert, so z.B. der hohe, hintere, labiale vokal [u] in [kũs̆t] - kunszt 'Kunstfertigkeit'. Die polnischen Nasalvokale werden nur vor dentalen Obstruenten voll nasal artikuliert, im Falle des Nasalvokals /õ/ auch im wortauslaut. Vor den anderen obstruenten werden sie nur leicht nasaliert zusammen mit einem der beiden Nasalkonsonanten artikuliert, z.B. [gwembok'i] - gzeboki 'tief' oder [bwont] - bzqd 'Fehler'. Bezüglich ihrer Nasalität unterscheiden sich die Nasalvokale vor nichtdentalen obstruenten also kaum von den anderen Vokalen, die vor Nasalkonsonanten stehen.

Die Konsonanten divergieren auf der phonetischen Ebene dann bezüglich [ \pm PALATAL], wenn sie vor palatalen bzw. nichtpalatalen Konsonanten stehen, bezüglich [ \pm STIMMHAFT] dann, wenn sie vor stimmhaften bzw, stimmlosen obstruenten oder im wortauslaut stehen; [v] und $[\bar{z}]$ bzw. $\{\dot{r}\}$ stehen nicht nur vor stimmhaften, sondern auch hinter stimmhaften Konsonanten, [f] und [s] dagegen nicht nur vor stimmlosen, sondern auch hinter stimmlosen Konsonanten. ${ }^{13}$

Sowohl die phonetisch als auch die morphologisch motivierten Alternationen werden in Regeln gefaBt. Die morphologisch motivierten Alternationen werden in Regeln gefaBt, die

(9) (a)die konsonantischen Alternationen betreffen;

(b) die vokalischen Alternationen betreffen.

Die Kennzeichnung des sog. Konsonantenwechsels in den slavischen Sprachen als eine die Methode erheblich vereinfachende Operation geht auf die Arbeiten von Isačenko (u.a. 1974, FuBn. S. 340) zurück. Sie vereint die sog. 1. und 2. - im Polnischen auch die sog. 3. - Palatalisierung mittels zweier Symbole, die auch in die synchronische Beschreibung eingesetzt werden können. ${ }^{14}$ So wird $2 . B$. der Wechsel $\{k\} z u\{\check{c}\}$ durch das Symbol $\left\{^{*}\right\}$ gekennzeichnet, der Wechsel $\{k\} z u$ \{\} dagegen durch das Sym- 
bol $\{$,$\} . Da der morphologisch motivierte Konsonantenwechsel$ auf der morphonemischen Ebene stattfindet, wird hier von einer

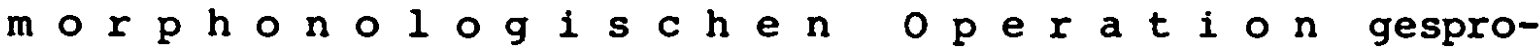
chen.

Als Folge dieser morphonologischen Operation Konsonantenwechsel ist in den meisten Fällen die morphologisch motivierte Alternation der Vokale zu sehen; daher wird auch in den Regeln, die den sog. Vokalwechsel betreffen, die Umgebung entsprechend berücksichtigt; vgl. (PL16) - (PL21).

Für die Annahme bzw. Nichtannahme der morphonologischen operation "Stern" ein Beispiel: In der Gruppe der berufsbezeichnenden Nomina stehen im Polnischen neben einander:

(10) (i) piekara, leḳarz, apteḱarz

'Becker, Arzt, Apotheker'

(ii) mleczarz, wilczarz, zapazczarz

'Milchhändler, Wolfsjäger, streichholzhersteller'

Wăhrend in $(10(i i))$ der Stammauslaut $\{k\} z u$ \{c\} wechselt - denn es heiBt miek-o, wilk, zapazk-a 'Milch, Wolf, Streichholz' bleibt der durch die gleichen Merkmale gekennzeichnete Stammauslaut in $(10(i))$ unverändert. Die Bedingungen für die Annahme bzw. Nichtannahme des Konsonantenwechsels sind zwar unterschiedlich, aber ausschlieblich auf der morphonemischen Ebene zu finden und festzulegen. Einerseits kann festgestellt werden, daB es sich in (1O(i)) - auBer Zekarz - um keine genuine polnische Wörter, sondern um Entlehnungen handelt, anderseits muB festgestellt werden, daB bestimnte polnische suffixe, wie z.B. -nik in dziennikgarz den Konsonantenwechsel auch nicht mitmachen. Die Wortbildungsregeln müssen also einerseits die Annahme, anderseits die Nichtannahme dieser morphonologischen Operation berucksichtigen, und dies aufgrund von Bedingungen, die auBerhalb der Phonologie zu finden sind.

Die Operationen "Stern" und/oder "Komma" werden nur an der Formativgrenze - vgl. (4(g)) - angenommen, d.h., daB der Konsonantenwechsel in der Wortbildung des Polnischen grenzmarkierende Funktion hat. Gleichzeitig ermöglicht die morphonologische operation die Assoziation zwischen lautlicher Repräsentation und Bedeutungsunterschied, indem sie den jeweiligen konsonantischen 
Alternanten mit der jeweiligen lexikalischen oder/und grammatischen Kategorle assoziativ verbindet.

Zu den morphologisch motivierten Alternationen gehören - wie bereits festgestellt - auch die Vokalalternationen des Polnischen. Wie beim morphologisch motivierten Konsonantenwechsel besteht auch zwischen den alternierenden Vokalen Korrelation. Doch im Gegensatz zu den alternierenden Konsonanten bestimmen die alternierenden Vokale keine Formativgrenze. Die Vokalalternationen stehen unmittelbar vor oder hinter der konsonantisch alternierenden oder nichtalternierenden Formativgrenze. Es läBt sich feststellen, daB die Vokalalternation z.B. a/e in miara / / mierny / mierzye / mierze 'MaB/mäBig/messen/(DAT, LOK SG) MaB' sowohl vor alternierendem als auch vor nichtalternierendem Konsonant durch den Alternanten $e$ repräsentiert wird. Daher erweist es sich als sinnvoll, nicht von alternierenden Konsonanten, sondern von einer konsonantischen Alternation auszugehen, und diese als Bedingung für die vokalische Alternation festzusetzen.

Für die morphologisch motivierten Alternationsregeln - vgl. (9) gilt also folgende Unterteilung in:

(11) (a)konsonantische Alternationsregeln, die - phonologisch gesehen - nicht umgebungsbedingt sind;

(b) vokalische Alternationsregeln, die ungebungsbedingt sind.

\subsection{WORTGRENZEN}

Voraussetzung für die morphonemische struktur eines Worts sind Formativgrenzen. Chomsky \& Halle (1968) gehen von Ketten (strings) aus, die mittels Grenzen (boundaries) und Klammern (brackets) - vgl. (1) und (2) - zu lexikalischen und grammatischen Formativen zusammengefügt werden. Paradigmatisch kennzeichnen die Grenzen und Klammern korrelierende, syntagmatisch koexistierende Morpheme, die als segmente und damit als Merkmalkomplexe verstanden werden und denen wiederum eine entsprechende phonologische Repräsentation zugeordnet wird.

Die Grenzen werden von Chomsky \& Halle $(1968,364)$ in Opposition zum segment notiert. Während das segment das Merkmal [+ SEGMENT] 
hat, wird die Grenze durch das Merkmal [- SEGMENT] gekennzeichnet. Da im Wort zwischen inneren und auBeren - vgl. (4(e), (f)) - Grenzen unterschieden wird, kann zwischen [ und [ \pm WORTGRENZE] unterschieden werden. Demnach gelten nach Chomsky \& Halle $(1968,371)$ folgende Grenzen:

$$
\begin{aligned}
& \text { FORMATIVGRENZE entspr } \cdot\left[\begin{array}{l}
- \text { SEGMENT } \\
+ \text { FORMATIVGRENZE } \\
- \text { WORTGRENZE }
\end{array}\right] \\
& \text { WORTGRENZE entspricht }\left[\begin{array}{l}
- \text { SEGMENT } \\
- \text { FORMATIVGRENZE } \\
+ \text { WORTGRENZE }
\end{array}\right]
\end{aligned}
$$

Der Nachteil der Definition der Grenzen in (12) ist u.a. der, daB der Zusammenfall von Wort- und Formativgrenze - vgl. (4(e))- nicht berücksichtigt ist.

Paulsson (1974,8f.) revidiert die Grenzen von Chomsky \& Halle (1968), indem er das Merkmal [ \pm ANFANGGRENzE] (initial boundany) einführt und die Wortgrenzen folgendermaBen definiert und kennzeichnet, wobei [ \pm AG] für [ \pm ANFANGGRENZE] und [ \pm WG] für [ \pm WORTGRENZE] stehen:

$$
\left[\begin{array}{c}
+A G \\
+W G
\end{array}\right]\left[\begin{array}{c}
- \\
-W G \\
-W G
\end{array}\right]\left[\begin{array}{c}
+ \\
-A G \\
-W G
\end{array}\right]\left[\begin{array}{c}
-A G \\
+W G
\end{array}\right]
$$

Demnach werden die Grenze zwischen Präfix und stamm durch '-', die Grenze zwischen stamm und suffix bzw. Infix durch 't', der Wortanfang durch '=' und das Wortende durch '\#' gekennzeichnet. 


\subsection{MORPHOLOGIE}

Gegenstand der Morphologie ist hier die morphemische Struktur des Worts im Hinblick auf die stellung der Wortsegmente zu einander sowie deren Funktion innerhalb der Wortbildung. Durch die Annahme der Wortgrenzen (13), die bezulglich ihrer stellung und in Opposition zum wortsegment bestimmt werden, ist die Möglichkeit gegeben, das wort - entsprechend der Behauptung (4(b)) - als Gruppe von Segmenten aufzufassen, die eine bestimmte Funktion erfüllen. Da - entsprechend (3) - jedes segment einem symbol komplexer Merkmale entspricht, ist es Aufgabe der Morphologie, Bedingungen zu schaffen, um bestimmte Merkmale für bestimmte Wortsegmente aufstellen $\mathrm{zu}$ können. Solch eine Morphologie erfüllt die Forderungen der morphologischen Komponente des Worts, die die Voraussetzungen für die zuordnung der morphonemischen Repräsentation schafft.

Die morphologische Komponente wird in die syntaktische Komponente einbezogen, da die zuordnung der morphonemischen Repräsentation und damit auch die Wortbildung innerhalb der Basis-struktur des satzes stattfinden.

\subsection{SYNTAKTISCHE UND MORPHOLOGISCHE KOMPONENTE}

In der Erweiterten Standard-Theorie - vgl. u.a. Radford (1981, 155,177) - setzt sich die Basis-struktur des Satzes zusammen u.a. aus: Phrasen-Struktur-Regeln, dem Lexikon, den lexikalischen Redundanzregeln, den Wortbildungsregeln und den lexikalischen Einsetzungsregeln. Der output der Satz-Basis bildet die Tiefenstruktur des Satzes, während der Transformationsteil sich aus verschiedenen Bewegungsregeln (movement rules) zusammensetzt und sein output die seichtstruktur (shallow structure) bildet, innerhalb welcher die Flektive zugeordnet werden.

Die Aufteilung der Regeln geht von folgenden Voraussetzungen aus: Der Satz ist hierarchisch als Gruppe von Konstituenten strukturiert. Jede Konstituente wird durch eine Kategorie bestimmt. Es wird zwischen lexikalischen Kategorien, die auf dem wort basieren, also zwischen $N, A, V, P, \ldots$, und Phrasen-Kategorien, also NP, AP, VP, PP,..., unterschieden.

Die Phrasen-Struktur-Regeln generieren abstrakte (wortleere) 
Phrasen-Marker. Anhand des Lexikons, das sich aus sämtlichen wortern einer Sprache zusammensetzt und nach lexikalischen Kategorien geordnet ist, werden die lexikalischen Einsetzungsregeln bestimnt; die lexikalischen Einsetzungsregeln bestimmen, welches Wort in welchen Phrasen-Marker einzusetzen ist.

Ein Phrasen-Marker entspricht einer bestimmten Menge (set) von Knoten (nodes), und es wird 2 wischen terminalen und nichtterminalen Knoten unterschieden. Jeder Knoten ist gekennzeichnet: Die nichtterminalen Knoten durch ein Kategorienmerkmal, die terminalen Knoten durch ein eingesetztes Wort.

Zwischen den Knoten eines Phrasen-Markers werden zweierlei Relationen unterschieden: a) ein knoten steht einem anderen knoten voran (precedence), d.h., das der eine Knoten links vom anderen Knoten steht; b) ein Knoten dominiert einen anderen (dominancel, d.h., daB ein knoten entlang den Verzweigungen

(branches) die nächsthöher gelegene stellung (Radford 1981,79

ff.) einnimmt. Wenn für den Satz die Basis (14) gilt:

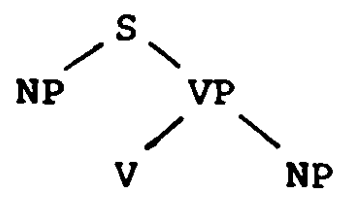

dann dominiert $S$ sowohl NP als auch VP, VP dominiert wiederum $V$ und NP; wăhrend NP der VP und V der NP voranstehen.

Dem Wort, das auf der Oberflächenstruktur 15 wohlgeformt - d.i. grammatikalisch richtig - entweder phonisch oder graphemisch repräsentiert erscheint, liegt bereits in der Basis-struktur des Satzes eine bestimmte struktur von Segmenten zugrunde, die als Formative fungieren; und es steht auBer $\mathrm{zweifel,} \mathrm{daB} \mathrm{min-}$ destens eins dieser Formative der lexikalischen Kategorie zugrunde gelegt werden muB, wenn die lexikalische Kategorie, neben der Phrasen-Kategorie, vorausgesetzt wird. Mit anderen Worten: Der lexikalischen Kategorie, durch die die Knoten der Satz-Basis gekennzeichnet sind, entspricht ein bestimmtes Formativ innerhalb der morphemischen struktur des Worts, das seine Einsetzung in diese Satz-Basis ermöglicht. Dieses Formativ ist Teil der Wortbildungsregel.

Es muB davon ausgegangen werden, daB eine lexikalische Kategorie einen terminalen Knoten dominiert, der durch eine, zunächst 
morphem- bzw. morphonemleere Gruppe von Segmenten w gekennzeichnet ist. Innerhalb $w$ befindet sich ein segment, das zunăchst allein durch Grenzen und entsprechend gekennzeichnete Klammern definiert, der lexikalischen Kategorie zugeordnet wird. 16 Damit erhält dieses segment den Status eines Formativs, dem eine entsprechende morphonemische Repräsentation zugeordnet werden kann.

Da diesem Formativ, das von der lexikalischen Kategorie dominiert wird, noch andere Segmente - ebenfalls mit der Funktion von Formativen - voranstehen, kann für $W$ ein $l$ e $x i k$ a $l i-$ $s \mathrm{c} h \mathrm{e} \quad \mathrm{F}$ or $\mathrm{m}$ a $t$ i $\mathrm{n}$ s $\mathrm{e} i \mathrm{l}$ angenommen werden. Der lexikalische Formationsteil betrifft die Wortbildung; er wird im Gegensatz zum $g$ r a $m$ m $t$ i $s c h$ e $n$ Formationsteil angenommen, der die Wortbeugung betrifft. Da diese beiden Teile für alle lexikalische Hauptkategorien, also für $N, A$ und $V$ gelten, wird für $W$ ein generalisierter Wort-(Formativ-)-Marker angenommen. 17

\subsubsection{GENERALISIERTER WORT-MARKER}

Der generalisierte W(ort)-Marker setzt sich aus einem lexikalischen Formationsteil (LFT) und einem grammatischen Formationsteil (GFT) zusammen. Dabei vereint LFT sämtliche lexikalische Formative, die als segmente in (3) definiert sind, während GFT den Teil des Worts bildet, der in der seichtstruktur - vgl. $\S 3.1$ - infolge der Kasuszuordnung durch das entsprechende Flektiv repräsentiert wird.

In Anlehnung an die Definition (2) gilt:

$\begin{aligned} W & \rightarrow \mathrm{Z} / \mathrm{X} \overline{\mathrm{GFT}}_{\mathrm{Z}}^{\mathrm{Y}} \\ \text { LFT } & \rightarrow \mathrm{LFT} \mathrm{K} \\ \mathrm{GFT} & \rightarrow \text { FL }\end{aligned}$

wenn $C$ für ein Symbol komplexer Merkmale, $K$ für lexikalische Kategorie, FL für Flektiv und $X \_Y$ für Umgebung stehen.

In Worten: $W$ wird in (15) durch einen generalisierten W-Marker $z$ in der Umgebung $X \ldots y$ ersetzt, wăhrend $z$ durch den lexikalischen Formationsteil und den grammatischen Formationsteil, der lexikalische Formationsteil durch ein Symbol komplexer Merkmale 
$C$ und die lexikalische Kategorie $K$, der grammatische Formationsteil dagegen durch ein Flektiv FL ersetzt werden.

$C$ vereint sämtliche Voraussetzungen für die zuordnung von $K$; $C$ und $K$ vereinen sämtliche Vorauseetzungen für die zuordnung der morphonemischen Repräsentation sowie für die Einsetzung in die Basis-struktur des Satzes. Die zuordnungen innerhalb des LFT geschehen mittels Regeln, die den ProzeB der Wortbildung beschreiben; die zuordnungen innerhalb des GFT geschehen mittels Flexionsregeln. ${ }^{18}$

Für das Polnische kann behauptet werden, daB LFT stets GFT voransteht; diese Behauptung gilt selbst für Komposita, wie z.B. wysok-o-czuz-y 'hochempfindlich', wo LFT aus zwei Wortstämmen besteht; denn der Vokal -o-, der diese beiden Wortstämme miteinander verbindet bzw. von einander trennt, entspricht keiner FL-Repräsentation, sondern stellt die Reprásentation eines noch $\mathrm{zu}$ bestimmenden Formativs dar.

Ebenso kann für das Polnische behauptet werden, daB C stets $\mathrm{K}$ voransteht; denn das Formativ, das die lexikalische Kategorie bestimnt, schliebt stets LFT ab; dies gilt auch dann, wenn $K$ durch ein Null-Morphem repräsentiert ist.

\subsubsection{INHÄRENTE MERKMALE}

Ausgehend von der Behauptung, daB der generalisierte W-Marker (15) eine morphonemleere Gruppe von segmenten erzeugt und dabei sämtliche Voraussetzungen für die zuordnung der entsprechenden morphonemischen Repräsentation enthält - vgl. $\$ 3.1$ und $\S 3.1 .1-$ - werden im Hinblick auf die zuordnung der lexikalischen Kategorie $\mathrm{N}$ der Wortbildung Merkmale zugrundegelegt, die den Status von inhärenten Merkmalen einnehmen. ${ }^{19}$

$$
\begin{array}{ll}
\text { N } & \rightarrow[+ \text { N, } \pm \text { APPELLATIVUM }] \\
{[+ \text { APPELLATIVUM }]} & \rightarrow[ \pm \text { INDIVIDUATIVUM }] \\
{[+ \text { INDIVIDUATIVUM }]} & \rightarrow[ \pm \text { BELEBT }] \\
{[- \text { APPELLATIVUM }]} & \rightarrow[ \pm \text { BELEBT }] \\
{[+ \text { BELEBT }]} & \rightarrow[ \pm \text { MENSCHLICH }] \\
{[- \text { INDIVIDUATIVUM }]} & \rightarrow[ \pm \text { ABSTRAKTUM }]
\end{array}
$$

Die inhărenten Merkmale (16) haben in Polnischen nicht nur syntaktische Funktion, indem sie z.B. bei der Bestimmung der Va- 
lenz bzw. in der Subkategorisierung eine Rolle spielen; sie haben auch morphologische Funktion, indem sie sowohl die zuordnung einer bestimmten Repräsentation von $K$ als auch die zuordnung einer bestimmten Repräsentation von FL bestimmen bzw. mitbestimmen. So ist $2 . B$. die zuordnung des Suffixes -arz in Polnischen ausschlieblich mit den inhärenten Merkmalen [+ BELEBT], [+ MENSCHLICH] sowie mit dem Genusmerkmal [+ MASKULINUM] verbunden. Innerhalb der Flexion entscheidet z.B. das Merkmal [+ BELEBT] bei den durch [+ MASKULINUM] gekennzelchneten Nomina über die Zuordnung des Flektivs - $a$ im GEN SG, während [- BELEBT] in der Regel die zuordnung des Flektivs - $u$ im GEN SG der Maskulina zur Folge hat. 20

Es wird also im Polnischen davon ausgegangen, daB die inhärenten Merkmale eng mit den Affixen verbunden sind. Da die Affixe morphonemisch repräsentierten segmenten entsprechen, die mittels phonologischer Merkmale dargestellt werden können, und da diese Segmente mit bestimmten inhärenten Merkmalen assozilert werden, gilt die Feststellung, daß bestimmte inhärente Merkmalkombinationen $\mathrm{zu}$ bestimmten phonologischen Merkmalbündeln im Verhältnis einer eindeutigen zuordnung stehen. Die inhärenten Merkmale sind also nicht auBersprachlich motiviert, sondern spiegeln die Affigierung im Polnischen wider.

Die Inhärenten Merkmale werden von den strukturellen Merkmalen getrennt. zu den strukturellen Merkmalen zählen z.B. die Merkmale der Kasus, der Personen, des Numerus u.s.w., die bei der Affigierung keine Rolle spielen, jedoch die Affigierung mittels inhärenter Merkmale voraussetzen; denn ein Nomen, wie z.B. owczarz 'Schafezüchter', kann erst dann mit dem GEN-SG-Flektiv -a versehen werden, nachdem das Suffix -arz den entsprechenden inhärenten Merkmalen gemäß dem Wortstamm zugefügt worden ist. Die inhärenten Merkmale haben demnach eine syntaktische Funktion, indem sie in der Subkategorisierung des Nomen z.B. bestimmte Adjektiva oder bestimmte Verben ausschlieben oder zulassen; so schliebt z.B. das Merkmal [+ MENSCHLICH] in owczarz das Verb szczekac 'bellen' sowie die qualitativen Adjektiva, wie z.B. gzeboki, pzytki 'tief, seicht' aus, wăhrend Verben, wie z. B. hodowat 'zuchten' nebst qualitativen Adjektiva, wie z.B. mqdry, gzupi, bogaty, biedny 'klug, dumm, reich, arm' von diesen 
Merkmal zugejassen werden. Neben der syntaktischen Funktion haben also die inhärenten Merkmale eine morphologische Funktion, die zum einen die Affigierung, zum anderen die zuordnung der entsprechenden Repräsentation des Flektivs betrifft.

Was die morphologische Funktion der inhärenten Merkmale innerhalb der Nominalisierung im Polnischen betrifft, so muBdie Feststellung gemacht werden, daß die inhärenten Merkmale (16) nicht ausreichen, um die Bedingungen für die zuordnung der unterschiedlichen K-Repräsentationen ausreichend differenziert darstellen $z u$ können; daher sind einige Uberlegungen zur wortsemantik notwendig. 
Gegenstand der Wortsemantik ist die Bedeutung des Worts im Hinblick auf die Wortbildung. Neben den phonologischen und morphologischen Merkmalen nehmen auch semantische Merkmale an der Wortbildung teil. Um diese $z u$ bestimmen, bedarf es einer Erläuterung der semantischen Komponente.

Der Versuch, die Semantik in die Standard-Theorie bzw. die Standard-Theorie in die Semantik einzubeziehen, wird von Katz \& \& Fodor (1963), Katz \& Postal (1964),.Chomsky (1965) und Weinreich (1966) gestartet, von Lakoff (bes. 1971), McCawley (u.a. 1968, 1969, 1972), Katz (1972) u.a. fortgesetzt, von Chomsky (1972) begleitet und von Chomsky (1975) in einigen Revisionsversuchen ausgewertet. Innerhalb der Erweiterten Standard-Theorie bietet Jackendoff (1975) für die wortbildung einige überzeugende Lösungen, Jackendoff (1977) besonders für die Syntax, wenngeleich seine $\bar{x}$-Konvention angesichts der Schwierigkeiten, die Kasus einzusetzen, für die slavischen Sprachen kaum geeignet $z u$ sein scheint. ${ }^{21}$

Die unterschiedliche Sicht der semantischen Komponente trennt die Uberlegenden in zwei Lager. Das eine Lager bilden die sog. "Transformationalisten", die bei der Satzerzeugung semantischen Strukturen den Vorrang einräumen; dabei erzeugt die Basis-Komponente mittels bestimmter Bedingungen abstrakte semantische Repräsentationen, von denen die Transformationskomponente ihren Ausgang nimmt, um die oberflächenstruktur zu erzeugen. Die Transformationsregeln werden mit den semantischen Regeln $z u$ einem Regeltyp vereint. Das andere Lager bilden die sog. "Lexikalisten", die an der kategorialen Basis-struktur mit Lexikon sowie syntaktischer und semantischer Komponente festhalten.

Im folgenden werden die beiden Lösungsversuche hinsichtlich der mir wesentlich erscheinenden Gedanken kurz erläutert.

\section{1 SEMANTISCHE KOMPONENTE}

Die Semantik-Theorie muB - nach Katz \& Fodor (1963) - formal und nicht intuitiv sein, um die Erfordernisse als Theorie zu erfüllen, während ihr Gegenstand nicht die ethnische, sondern die natürliche Sprache, nicht die Performanz, sondern die 
Sprachkompetenz sein sollten. In diesem Sinne wird auch zwischen Sprachtheorie und sprachbeschreibung unterschieden. Während die Sprachbeschreibung u.a. die Aufgabe erfüllt, Sprachtheorien $\mathrm{zu}$ bestätigen oder $\mathrm{zu}$ verwerfen, verallgemeinert die Sprachtheorie die Beschreibung verschiedener Sprachen. Ziel der Sprachtheorie ist das Aufstellen sprachlicher Universalien oder das verschiedenen Sprachen Gemeinsame und damit das Aufstellen eines allgemeinen Musters für die Sprachbeschreibung. Die Sprachtheorie setzt sich aus einer Syntaxtheorie - im Sinne der generativen Transformationsgrammatik - einer Phonemtheorie sowie einer Semantiktheorie zusammen.

Ziel der Semantiktheorie ist das Aufstellen von Regeln, deren Sinn es ist, in einer endichen Anzahl von Schritten die Bedeutung von zusammengesetzten Ausdrücken festzulegen, wenn die Bedeutung einfacher Ausdrücke gegeben ist. Die Bedingung dafür ist der idealisierte Sprecher, dessen semantische Kompetenz sich zeigt in:

(17)(a) der Sprachverwendung durch Bestimmung von 2 ahl und Inhalt der Lesarten eines Satzes;

(b) der Festlegung semantischer Anomalien;

(c) der Entscheidung iber Paraphrasen-Beziehungen;

(d) der Bezeichnung jeder anderen semantischen Eigenschaft oder Beziehung, die bei der Fähigkeit des Sprechers eine Rolle spielen.

Die semantischen Regeln haben Rekurrenz-Charakter und enthalten folgende Bedingungen:

(18)(a) Bildung oder Aufzählung einfacher Elemente (Eingangsbedingung I:

(b) Bildung weiterer Elemente aus einfachen Elementen mit bestimmten gleichen Eigenschaften (Induktionsbedingung).

Die semantischen Regeln enthalten Bedeutungen, die auf psychische Seinsverhalte bzw. Entitäten hinweisen, deren Identität Bedingung für das verstehen ist.

Die Eingangsbedingungen stellen die Bedeutung der einzelnen Morpheme bzw. Wörter fest. Jede Eingangsbedingung hat die Gestalt einer Lexikonposition. In jeder Lexikonposition sind an- 
gegeben :

(19)(a) ein entsprechendes Lexem;

(b) die grammatische (=lexikalische) Kategorie; bei Mehrdeutigkeit alle Kategorien;

(c) die Information über die Bedeutung, die in Gruppen eingeteilt hinter der Kategorie steht.

Als Beispiel aus dem Polnischen kann z.B. folgendes Verb genannt werden:

(20)

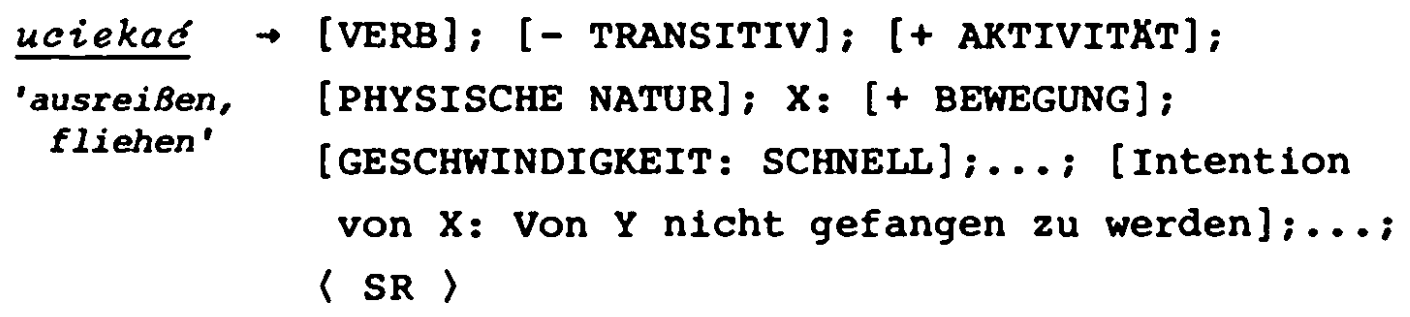

wenn X für subjekt, Y für objekt und SR fur Selection Restriction stehen. SR stellt fest, mit welcher Art von Ausdruck und mit welcher seiner Bedeutung ein Ausdruck zu einem Ganzen verbunden werden kann.

Jede Lexikoneinheit setzt sich - (20) entsprechend - zusammen aus:

(21)(a) syntaktischen Markern;

(b) semnatischen Markern;

(c) Selektionsbeschrănkungen (=SR).

Neben den semantischen Merkmalen werden Distinktoren ldistinguishers I angenommen, deren Aufgabe es ist, anzugeben, was in der der jeweiligen Bedeutung einmalig ist.

Am Beispiel des Polnischen werden in (22) - in Entsprechung $z u$ Katz \& Fodor $(1963,186)$ - anhand des Nomen kawaler die Lexikoneintragungen und die Distinktoren, die in diesem Fall die Lesarten bestimmen, veranschaulicht. Dabel wird bereits der Kritik Weinreichs (1966) bezüglich der Notationsweise Rechung getragen, indem diese insofern okonomischer gehandhabt wird, als 2.B. Wiederholungen von Merkmalen in Baumgraph vermieden werden. Die zuordnung der Distinktoren geschieht anhand DOR. 
(22)

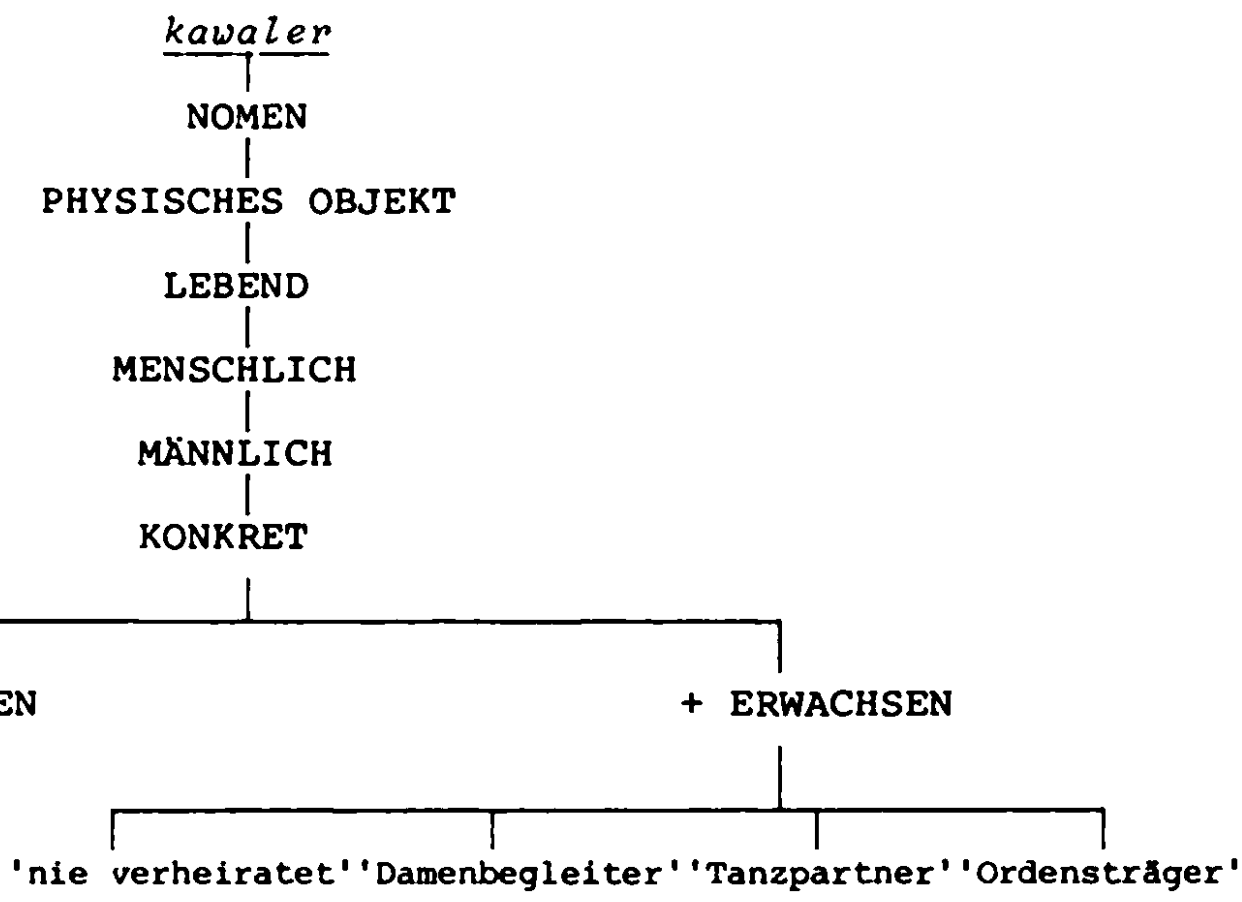

Katz \& Fodor entsprechend hat ein Ausdruck eine zusamengesetzte Begriffsstruktur, deren Darstellung Aufgabe des semantischen Merkmals ist. AuBerdem enthält jeder Ausdruck Selektionsbeschränkungen, die seine Einbeziehung in den Kontext bedeutungsmäßig bestimmen. ${ }^{22}$

Lexikon und Regeln dienen einzig der Interpretation von generierten Satzstrukturen; daher u.a. die Einbeziehung der grammatischen Kategorie. Dabei wird - entsprechend Chomsky (1965) - zwischen Oberflächen- und Tiefenstruktur unterschieden; die Bedeutung wird bereits auf der vortransformationellen Ebene, also innerhalb der Basis-Komponente des Satzes angesetzt und darf von den Transformationen nicht verändert werden.

Weinreichs (1966) Kritik wendet sich hauptsächlich gegen Katz \& \& Fodor (1963). Ich versuche diese kurz zusammenzufassen, weil Weinreich die Einbeziehung seiner Semantik-Theorie in die standard-Theorie weitgehend auf dieser kritik aufbaut. Weinreich schafft die Grundlegungen für eine auf der Semantik und nicht auf der syntax basierende Grammatik.

Der theoretische Status der Semantik-Theorie von Katz \& Fodor ist für weinreich unklar; dies bezogen auf die semantische vs. syntaktische Ebene. Er wirft Katz \& Fodor vor, sie hätten die Beziehungen zwischen den Paraphrasen nur flüchtig behandelt und ihre Theorie könne den Inhalt der Lesarten gar nicht rich- 
tig erfassen. In Wirklichkeit - so Weinreich - befasse sich die Semantik-Theorie von Katz \& Fodor mit einem beschränkten Teil der semantischen Kompetenz, d.h. mit der Auffindung von Anomalien und mit der Bestimmung einer gewissen Anzahl von Lesarten eines Satzes. Als Fehler sieht weinreich an, daB die Theorie von Katz \& Fodor nur die Bildung eines eindeutigen (bzw. weniger mehrdeutigen) Ganzen aus mehrdeutigen Teilen erklärt, wobei die Gefahr unbeachtet bleibt, daB Differenzierungen von Unterbedeutungen im Lexikon unbegrenzt fortgesetzt werden können. Als ziel einer Semantik-Theorie sieht Weinreich die Garantie formulierter Festlegungen eindeutiger Tiefenstrukturen einschlieblich der lexikalischen Komponente, um von ihnen aus einen komplexen Ausdruck aus bekannten Bedeutungen seiner Komponente $\mathrm{zu}$ erklären.

Die semantischen Merkmale sind für Weinreich unteilbare Einheiten. Die Mehrdeutigkeit kann ökonomischer notiert werden, wenn die Distinktoren nach dem letzten semantischen Merkmal verzweigt - vgl. (22) - werden. Nebenbei zweifelt Weinreich die Notwendigkeit von Distinktoren an. Das Hinzufügen eines semantischen Merkmals bedeutet Subkategorisierung. ${ }^{23}$

Bezogen auf den Satz sieht weinreich das ziel einer Semantik-Theorie darin, daB die Bedeutung eines satzes mit einer bestimmten struktur aus den vollständig bestimmten Bedeutungen seiner Teile abgeleitet wird. Den Ausgang dazu bildet die Standard-Theorie; ihr gemäB generiert die Basis Tiefenstrukturen von Sätzen; der Transformationsteil besteht aus obligatorischen Transformationen, die auf die Tiefenstruktur einwirken; die kategoriale Komponente generiert präterminale Ketten; diese setzen sich zusammen aus: $P$ : phonologischen Merkmalen, $Q$ : syntaktischen Merkmalen sowie $\mu$ : semantischen Merkmalen. Die Tiefenstruktur ist frei von Mehrdeutigkeiten durch Lexikoneinträge.

Die Merkmale sieht Weinreich als Mengen; wobei er zwischen geordneten und nichtgeordneten Mengen unterscheidet. Geordnete Mengen von Merkmalen bilden eine Konfiguration, die nichtgeordneten Mengen von Merkmalen bilden eine Verkettung (clusten). So kann 2.B. die Abbildung von a auf b im sinne einer Konfiguration im Polnischen für krzesto 'Stuhl' einer Abbildung des 
Merkmals [MOBEL] auf [SITZEN] entsprechen, während für syn 'Sohn' die Merkmalverkettung [MANNLICH; NACHKOMME] gilt. Die Konfiguration entspricht einer eindeutigen Zuordnung.

Konfiguration und Verkettung sind unter bestimmten Voraussetzungen mit einander kombinierbar; so können $2 . B$. Verkettungen in Konfigurationen ebenso wie Konfigurationen in Verkettungen vorkommen; desgleichen sind Konstruktionen möglich, die nur aus einer Verkettung oder nur aus einer Konfiguration bestehen. Mittels Konfiguration und Verkettung versucht Weinreich sämtliche semantische Erscheinungen, die für die Bildung eines generialisierten $P$-Markers infrage kommen, zu formalisieren. ${ }^{24}$

Chomskys Unterscheidung zwischen inhärenten und kontextuellen syntaktischen Merkmalen sowie deren Beziehung zu einander führen Weinreich zu folgenden Behauptungen:

(23) (a) Selektionsmerkmale eines worts $w$ spiegeln inhärente syntaktische Merkmale von wortern im Kontext wider;

(b) Merkmale der strikten Subkategorisierung spiegeln die grammatischen Kategorien von wörtern im Kontext wider.

Dabei werden die Selektionsmerkmale den übertragungsmerkmalen (transfer markers) gleichgesetzt, die bel Katz \& Postal (1964) den Selektionsbeschränkungen entsprechen; so kann z.B. das Merkmal [+ BELEBT] von engl. educate auf ein entsprechendes $N$ oder 2.B. das Merkmal [+ ZEIT] von engl. during auf the day ubertragen werden. Die Voraussetzung für solche Ubertragungen ist u.a. die Annahme eines Merkmals $k$, das als semantisches Merkmal der Verursachung angenommen wird. Bezogen auf das polnische gilt z.B.:

(24) WENN : (a,b) auf Art und Weise

$(f, g)$ Subjekt; verursacht, führt herbei zustand,...

$(m, n)$ objekt, in dem $(m, n)$ und $(u, v)$ verkettungen bilden

$(u, v)$

UND 2.B.:

$$
\begin{aligned}
& \text { (a, b) chetnie 'gern' } \\
& (\mathrm{f}, \mathrm{g}) \text { Piotr 'Peter' } \\
& \text { (m, n) wbdke 'schnaps' }
\end{aligned}
$$


DANN GILT:

$$
\text { (u,v) pije 'trinkt' }
$$

SUBJEKT + V + OBJEKT - Konstruktion =

= Konfiguration $(a, b, f, g k \rightarrow m, n, u, v)$

IM SATZ :

Piotr chetnie pije wodke. 'Peter trinkt gern schnaps.'

Das Merkmal der Verursachung $k$ wird in (24) dem Subjekt und der Art und Weise zugeordnet, um den Agens als Verursacher der Tätigkeit zu kennzeichnen, die mit dem objekt eine Konfiguration bildet. Als Ubertragungsmerkmal von $(u, v)$ auf $(m, n)$ kann das Merkmal [+ FLUSSIG] angenommen werden.

Ferner führt Weinreich einen Kalkulator ein. Dieser Kalkulator

(25)(a) verteilt gewisse semantische Merkmale entlang den Verzweigungen des Baumgraphs;

(b) kennzeichnet widersprüche zwischen bestimmten semantischen Merkmalen im Satz;

(c) verschmilzt redundante Merkmale;

(d) uberträgt manche Merkmale von einem Morphem auf ein anderes:

(e) tilgt bestimmte Teile des zugrundeliegenden Markers.

Schließlich wird jeder Lexikoneintrag als ein Morphem gesehen, wobei die Morpheme in eine zentrale Morphemklasse von $\mathrm{N}, \mathrm{V}, \mathrm{A}$, ADV,... mit nur einem unterscheidenden Merkmal und in eine randliche Morphemklasse von Artikel, Präposition, Konjunktion, ... ohne ein unterscheidendes Merkmal, jedoch mit der Aufzählung von semantischen Merkmalen eingetellt werden. Demnach wäre z.B. der Lexikoneintrag für engl, with entsprechend $P, Q, \mu$ : : /wiס/, [ [ P ] ], [+ INSTRUMENTALITÄT].

Die auf der Semantik und nicht auf der syntax basierende Grammatik von McCawley und Lakoff geht von Basis-Regeln aus, die semantische Repräsentationen erzeugen. Mittels eines Systems von prä- und postlexikalischen Transformationen sowie lexikalischen Einsetzungen werden diese semantischen Repräsentationen in Oberflächenstrukturen überführt. Da in der Auffassung von McCawley und Lakoff die Strukturen, die der standard-Theorie zugrunde liegen, auf die symbolische Logik gestutzt sind, entsprechen die Kategorlensymbole $S, N P, V$ der Proposition, dem 
Argument und der Prädikat. $25 \mathrm{~S}$ ist Anfangssymbol, wăhrend NP und $v$ die Endsymbole der semantischen Repräsentation darstellen.

Chomskys (1965) Formations- bzw. Ersetzungsregeln werden durch Bedingungen fur die zulässigkeit von verzweigungsknoten Inode admissibility conditions) - McCawley (1968,244-253) - ersetzt. Diese Bedingungen werden auf fertige strukturbăume angewandt, um deren wohlgeformtheit festzustellen. Die NP wird durch Variable oder durch Konstanten repräsentiert. Variable mússen durch quantoren gebunden werden (McCawley 1972). Quantoren entsprechen a) Prädikaten, die uber Sătze prädiziert werden, und b) intransitiven Verben, die über eine NP, welche eine bestimmte Menge von Propositionen bezeichnet, prădiziert werden. Die Oberflächenstruktur entspricht einer linearen Anordnung von Bedeutungselementen. Die syntaktischen und die semantischen Beziehungen werden aus ihrer verkettung herausgelesen und auf die elementaren Beziehungen, wie Subjekt-Von, objekt-Von, Prădikation,.... die zwischen den Bedeutungselementen bestehen, zurückgefuhrt. Da die semantischen Repräsentationen als Endsymbole keine lexikalischen, sondern semantische Einheiten enthalten - die an lexikalischen Einheiten orientiert sind -, werden lexikalische Einsetzungsregeln aufgestellt.

Lyons $(1977, \$ 10.5)$ stellt die standard-Theorie mit der integrierten Semantik-Theorie von Katz et al. der generativen Semantik von Lakoff (1971) gegenüber. Er stellt u.a. fest, daB die syntaktisch orientierte Methode Chomskys die Unterscheidung zwischen der Basis und den semantischen, den Transformations-, sowie den phonologischen Regeln voraussetzt, wobei die semantischen Regeln zur semantischen Interpretation fuhren. Die generative Semantik bedarf keiner Unterscheidung zwischen der Tiefenstruktur und der semantischen Interpretation. Was das Lexikon betrifft, so enthalt bei Chomsky (1965) die Tiefenstruktur alle lexikalischen Einheiten, die auf der oberflachenstruktur erscheinen. Für die generativen Semantiker ist die Lexikalisierung ein Teil des transformationalen Prozesses. Und Lyons komnt u.a. zum SchluB, daB sich die Transformationalisten von den Nicht-Transformationalisten - sprich: Lexikalisten - durch eine unterschiedliche Auffassung von Meaning unterscheiden. 
Chomsky (1969) geht davon aus, das es kelne Theorie der semantischen Repräsentation gebe, die wohldefiniert sei und auf die man sich in der standard-Theorie stützen könne. Er bezieht die semantische Komponente explizite in seine standard-Theorie anhand eines Systems von Regeln semantischer Interpretation ein; dies neben der kontextfreien kategorialen Komponente und dem Lexikon, die die Basis der Grammatik bilden. Das Lexikon beschreibt er als eine Klasse lexikalischer Eintragungen. Jede dieser Eintragungen spezifiziert die grammatischen (d.i. phonologischen, semantischen und syntaktischen) Eigenschaften eines lexikalischen Elements. Jede lexikalische Eintragung enthät eine bestimnte Menge von Transformationen, die das jeweilige Element des Lexikons (d.i. den Merkmalkomplex, der dieses Element konstituiert) in P-Markern einfuhrt. Wird die Bedingung der Unterscheidung zwischen lexikalischer und nichtlexikalischer Transformation erfült, so kann eine Klasse postlexikalischer strukturen definiert werden. Diese werden mittels seman$t$ ischer Regeln in einer semantischen Repräsentation dargestellt und bilden die Tiefenstruktur. Die Tiefenstrukturen enthalten samtliche lexikalischen Elemente samt einem Komplement grammatikalischer Merkmale. Die semantische Interpretation eines Satzes wird durch intrinsic semantic content seiner lexikalischen Elemente, sowie durch die Art, wie sie innerhalb der Tiefenstruktur in Beziehung gebracht werden, bestimnt. In diesen Sinne bestimmen laut Chomsky - nach Katz \& Postal (1964) - Tiefenstrukturen die semantische Repräsentation mittels Regeln der semantischen Interpretation.

\subsection{TEILAUSWERTUNG}

Weinreichs Unterscheidung zwischen Konfiguration und Verkettung stellt einen Vorschlag dar, die bei Katz et al. ungeordneten Merkmale zu ordnen. Sein Kalkulator - vgl. (25) - trăgt u.a. der Annahme von Katz et al. Rechnung, daB der Ausdruck einer zusammengesetzten Begriffsstruktur entspricht, deren Darstellung Aufgabe der semantischen Merkmale und Distinktoren ist. Der Kalkulator trägt aber auch Rechnung den selektionsbeschränkungen, die feststellen, mit welcher Art von Ausdruck und mit welcher seiner Bedeutung ein Ausdruck $z u$ einem Ganzen verbunden 
werden kann. AuBerdem übernimmt auch der Kalkulator die Funktion der Katzschen Projektionsregeln, die u.a. semantische sinnlosigkeit bei grammatikalischer Korrektheit erklären sollen. 26

Zweifel an der Notwendigkeit der von Katz et al. angenommenen Distinktoren werden von Weinreich zwar geăuBert, jedoch nicht belegt. Wollte man eine Bedeutungsstruktur ohne einen "semantischen Rest" bestimmen, so hiebe dies, ein in sich.abgeschlossenes System von binären Merkmalen zu schaffen, in dem die Distinktoren ausnahmslos durch binäre Merkmale ersetzt wirden. ${ }^{2}$ Lo gisch gesehen wäre vielleicht - und davon lassen sich die sog. Transformationalisten leiten - die Schaffung eines abgeschlossenen Systems von Bedeutungen, die sämtlichen potentiellen Aussagen zugrunde liegen, mittels Quantoren und Konjunktionen möglich. 28 Die Bedeutung des Worts wäre dann von Prädikat-Argument-Strukturen getragen, die die Bedeutungserklärungen, wie sie bereits $2 . T$. in den wörterbüchern $z u$ finden sind, ablösen würden; dies jedoch mit dem Unterschied, daB die Prädikat-Argument-Strukturen Elemente eines systems wăren, was von den ublichen Erklärungen in den einschlägigen wörterbüchern nicht behauptet werden kann. ${ }^{29}$ Angenommen solch ein system stünde zur verfügung, so würde sich u.a. die Frage erheben, wie dieses Bedeutungssystem in die Wortbildung einzusetzen wäre; entweder mübten die Prädikat-Argument-Strukturen durch ein kompliziertes system von Symbolen ersetzt werden, das sicherlich dem Prinzip der Einfachheit widerspräche, oder jedes wort mulbte als Endprodukt einer entsprechend transformierten Prädikat-Argument-struktur verstanden werden.

Was das Katzsche System von Merkmalen und Distinktoren betrifft, so läbt sich zu seiner Rechtfertigung aus lexikologischer sicht der Standpunkt Coserius $(1973,105)$ anführen, nämlich:

(26) Ausgangspunkt für die Wahl der Methodik ist die Tatsache, daB der Wortschatz nicht in Ganzen, sondern nur partiell strukturiert ist.

Wenngleich Coseriu den strukturalistischen standpunkt vertritt, der auf der Auffassung des Zeichens als Einheit von Bezeichnendem und Bezeichnetem beruht, wird diese Behauptung meinen Vorschlägen zur Wortbildung im Polnischen zugrunde gelegt. Dabei 
wird das Merkmal als definiertes Element eines Merkmal-Inventars mit Systemcharakter verstanden, das im Hinblick auf die Nominalisierung im Polnischen aufgestellt wird; der Distinktor wird als Lesart verstanden, wobei nicht ausgeschlossen wird, daß er ebenfalls als Merkmal eingesetzt werden kann, sobald ein Merkmalkomplex für eine bestimmte Wortgruppe aufgestellt wird, in dem das Vorhandensein des von ihm bezeichneten Teils der Bedeutung binär markiert oder unmarkiert ist.

Was die Prädikat-Argument-Struktur betrifft, so kann festgesetllt werden, daß sie den meisten Nomina im Polnischen als Paraphrase zugrunde gelegt werden kann; dies jedoch nicht als prätransformationelle semantische Komponente, deren Endprodukt das entsprechende Nomen ist, sondern als bloBe Veranschaulichung des markierten Teils der Wort-Basis, der in Gestalt von binären Merkmalen über die Zuordnung eines bestimmten Affixes entscheidet. Als solch eine Paraphrase kann z.B.

Ten, co gra 'Derjenige, der spielt'

im voraus genannt werden, die in Gestalt der Merkmale $[+\mathrm{N}$;

+ BELEBT; + MENSCHLICH; + TÄTIGKEIT] u.a. die zuordnung der K-Repräsentation -acz zur Folge hat und das Nomen gracz 'Spieler'bilden läBt. Ob es sich dabei um einen 'professionellen' oder um einen 'Amateurspieler' handelt spielt bei dieser Affigierung keine Rolle. Die Unterscheidung zwischen dem 'Beruf' und 'Nicht-Beruf' wäre die Aufgabe eines Distinktors, der bei der Bildung der Nomina agentis im Polnischen - und dies kann man hier vorwegnehmen - kaum eine Funktion hat.

Das gleiche gilt dann, wenn die Paraphrase erweitert wird, nämlich:

$$
\text { Ten, co gra na gitarze 'Derjenige, der (auf der) Gitarre }
$$

Diese Paraphrase entspricht den Merkmalen u.a. [+ BELEBT; + MENSCHLICH; + TATIGKEIT], und es wird die K-Repräsentation von -ist-a in gitarzysta 'Gitarrist' hinzugefügt. Der Unterschied zwischen beiden Nomina ist u.a. auf der morphosyntaktischen Ebene festzustellen, nämlich auf der Unterscheidung zwischen DEVERBAL und DENOMINAL, während die Funktion der Merkmale ebenfalls eine ausschlieblich morphosyntaktische ist, die 
nicht nur auf die Wortbildung beschränkt bleibt, sondern auch -

- vgl. §3.1.2 - für die Wortbeugung wichtig ist. 
5.0 FOLGEN FUR DIE WORTBILDUNG

Der Streit zwischen den beiden "Schulen" - vgl. $\$ 4.0-\S 4.2$ - setzt sich auch in der Auffassung der Wortbildung fort und führt letztlich - durch Chomsky (1970) - zu einer Revision der Standard-Theorie, die sich auf die sog. $\bar{x}$-Ronvention - siehe u.a. Jackendoff (1977) - stützt. ${ }^{30}$

Da die Phrasenstruktur-Syntax (=Standard-Theorie) nur eine Phrasenprojektion für jede gegebene lexikalische Kategorie zuläBt und damit die Einsetzung der Wortbildung in die Satz-Basis erschwert, werden die Phrasenstruktur-Băume durch Dependenz-Bäume ersetzt, die eine Einsetzung der Wortbildung in die Satz-Basis - vgl. $\S 3.1$ - erleichtern. ${ }^{31}$ Die Dependenz-Bäume erfülen auBerdem die Forderung nach Nichtüberschneidung der Verzweigungen, dies nach den wohlgeformtheitsbedingungen (node well formedness conditions), gemäB welcher die Theorie den Fakten der natürlichen Sprache Rechnung tragen muB. Die Dependenz-Bäume bilden auch den Rahmen für die Aufstellung einer auf Merkmalen basierenden Syntax (feature-based-syntax): vgl. Radford $(1981,101 \mathrm{f}$.$) .$

Die Grundlegungen für die Wortbildung schafft ebenfalls Chomsky (1970), wobei er anhand des Englischen die sog. Lexikalisten-Hypothese gegen die sog. Transformationalisten-Hypothese überzeugend verteidigt, wenngleich er den Transformationalisten einige begründete zugeständnisse macht. Es schlieben sich u.v.a. an: Halle (1973), Jackendoff (1975), Aronoff (1976) sowie Laskowski (bes. 1981).

\subsection{CHOMSKY (1970)}

Für das Problem der Nominalisierung ist die Syntax der angemessene Untersuchungsbereich, da die Nominalisierung mit problemen des Kontextes verbunden ist. In der Standard-Theorie (1965) wird die Nominalisierung mittels einer Transformation durchgeführt, d.h. es werden z.B. nicht ein $V$ und nicht ein $A$, sondern ganze Phrasen nominalisiert. ${ }^{32}$ In dieser Theorie treten nach Chomsky zwel schwierigkeiten auf, erstens müBten marginal- bzw. quasiproduktive Wortbildungsvorgänge berücksichtigt werden, die bestimmten Beschränkungen unterliegen, zweitens müBte die No- 
minalisierung wie jede andere Transformation in der Tiefenstruktur angekündigt werden, so daB Probleme der Basis auftreten, die den Versuch sinnvoll erscheinen lassen, die Nominalisierung grundsätzlich in die Basis zu verlegen.

Chomskys Ausgangsfrage lautet: Werden nominale Ausdrücke aus den dazugehörenden wörtern transformationell abgeleitet, oder werden sie als selbständige Einheiten (items) in den lexikalischen Bereich der Basis einbezogen (S.188).

Bei der Bildung des Gerundium im Englischen akzeptiert Chomsky die Transformationalisten-Hypothese, der gemäB das Gerundium von einem darunterliegenden Satz abgeleitet wird (S.187); denn hier wird der V-Charakter beibehalten, wie wenn es sich um ein Partizip handelte, das ebenfalls nicht die innere struktur einer NP haben kann. Die Kombination mit einem DET(erminator) ist ausgeschlossen - nicht aber mit der Subjekt-NP des zugrundeliegenden Satzes mit Possessivus; dies in den Beispielen ${ }^{33}$ :

(27) (i) John is eager to please

(ii) John's being eager to please...

(iii) John's eagerness to please...

(27(ii)) enthält das Gerundive-Nominal, (27(iii)) das abgeleitete Nominal.

Bei dem abgeleiteten Nominal sieht es anders aus. Hier schafft die Transformationalisten-Hypothese Veränderungen auf der semantischen Ebene. Dazu das englische Beispiel, dem ich das entsprechende polnische Beispiel hinzufüge:

(28) (i) John is easy to please...

(ii) John's being easy to please...

(iii) *John's easiness to please...

(29) (i) Jana Zatwo (mozina) ucieszye

'Hans kann man leicht erfreuen'

(ii) (X uciesza Jana Zatwo)

' $x$ erfreut Hans leicht'

(iii) ( $X$ moze Jana Zatwo ucieszyr!)

' $X$ kann Hans leicht erfreuen'

(iv) $x$-a Zatwosc ucieszenia Jana

' $x$-ens Leichtigkeit Jan zu erfreuen'

(v) * Jana Zatwose ucieszenia

'Hansens Leichtiqkeit zu erfreuen' 
Das Passiv wird in $(29(1))$ durch die unpersönliche Form ersetzt, wobei die Relaislerung von mozina 'man kann' fakultativ ist. $(29(i i))$ und $(29(i i i))$ sind die Realisierungen von $(29(1))$ im Aktiv, wăhrend $(29(i v))$ und $(29(v))$ in etwa den englischen Beispielen in (28) entsprechen. Dabei zeigt (29(v)), das es ebenso wie das englische Beispiel (28(11i)) von den davorstehenden Beispielen nicht ableitbar ist.

Chomsky schlägt vor, abgeleitete Nominale als selbständige Lexikoneinträge $\mathrm{zu}$ behandeln.

Da abgeleitete Nominale ein weiteres Spektrum von Ergänzungen beinhalten können, werden von Chomsky folgende Regeln für die kategoriale Komponente der Basis (S.195) aufgestellt:

$$
\begin{aligned}
\mathrm{NP} & \rightarrow \mathrm{N} \text { COMP } \\
\mathrm{VP} & \rightarrow \mathrm{V} \text { COMP } \\
\mathrm{AP} & \rightarrow \mathrm{A} \text { COMP } \\
\mathrm{COMP} & \rightarrow \mathrm{NP}, \mathrm{S}, \mathrm{NP} \text { S, NP PP, PP PP,... }
\end{aligned}
$$

Aus (30) geht hervor, daB jede durch eine lexikalische Kategorie gekennzeichnete Phrase durch eine entsprechende Kategorie, die sie kennzeichnet, und ein komplement comp ersetzt werden kann. Dementsprechend gilt für abgeleitete Nominale die Basis-struktur:

(31) $\left.\complement_{\mathrm{NP}} \mathrm{DET}-\mathrm{N}-\mathrm{COMP}\right]_{\mathrm{NP}}$ be $\left[_{\mathrm{PRAD}} \Delta\right.$ ] $\mathrm{PRAD}$ Chomsky zieht den SchluB, daB Nomina im Prinzip dieselben distributionalen Eigenschaften (distributional properties) haben können wie Verben und Adjektive, Eigenschaften also, die folglich den lexikalischen Kategorien zuzuordnen (s.198) sind; $\mathrm{m}$. a. W.: Bestimmte Erscheinungen können immer mit mehr als einer oberklassenkategorie auftreten, es müssen nur die jeweiligen, dazu gehörenden kontextuellen Gegebenheiten formuliert werden. Deshalb sind auch die Verbal-Substantive bei Chomsky keine Produkte von Transformationen, sondern Produkte der Nachstellung des Agens (Agent-Postposing) folgender Form (S.202f.):

$$
\mathrm{DET}-\mathrm{N}-\mathrm{NP}-\text { by } \Delta
$$

Wenn (32) entsprechend notiert wird:

$$
\text { the enemy's - [ destroy, + N] - the city - by } \Delta \text {, }
$$


dann ergibt (33) das Verbal-substantiv distruction; also wird the destruction of the city by the enemy von the enemy's destruction of the city abgeleitet. Daraus geht hervor, dab nicht die Nominalisierung Gegenstand der Transformation ist, sondern die Movement-Regel der Nachstellung des Agens (s.203f.).

Die Entsprechung zu (33) im Polnischen lautet:

$$
\text { wrogow - [ zburz_, + N] - miasta - przez } \Delta
$$

In (34) ist also das $N$ wrogow '(GEN PL) Feinde' mit dem Symbol $\Delta$ austauschbar.

Aufgrund der Unterscheidung $z$ wischen verăuBerlichem und nichtveräuBerlichem Besitzverhältnis lalienable and unalienable possession) - (S.200) - wird für das nichtveräuBerliche Besitzverhältnis folgende Ersetzung von DET im Englischen bestimmt ${ }^{34}$ :

$$
\begin{aligned}
& \text { (35) (i) DET } \rightarrow \text { (PREARTICLE OF) ART (ICLE) (POSTARTICLE) } \\
& \text { (ii) ART } \rightarrow \text { [ } \pm \text { DEF, (NP) ] }
\end{aligned}
$$

Die Ersetzung $(35(i 1))$ ist notwendig, um anstelle einer possessiven Nominalphrase, die nach der Regel der Umstellung des Agens (32) aus der DET-Position entfernt worden ist, das Merkmal [ \pm DEF] erscheinen $z u$ lassen (S.207); dies ist gleichbedeutend mit einer Erweiterung des Merkmalbegriffs. Danach werden die lexikalischen Kategorien als Merkmale im Lexikon, aber auch als Kategorien der Basis aufgefaBt. ${ }^{35}$

Schlieblich wird der Unterschied zwischen Kategorie und Merknal aufgehoben, indem die Kategorie selbst als Merkmal formuliert wird. Und hier liegt der Ausgangspunkt für die $\bar{x}$-Konvention (S.210), für die allgemein gilt:

(36) (i) $\overline{\mathrm{x}} \rightarrow \mathrm{X} \ldots$

(ii) $\overline{\bar{x}} \rightarrow$ [SPEC, $\bar{x}] \bar{x}$

(36(i)) entspricht den Ersetzungen (30). Das Merkmal [SPEC] steht sowohl $\overline{\mathrm{N}}$ als auch $\overline{\mathrm{V}}$ voran, indem es z.B. als quantifier, als Degree - vgl. Jackendoff (1977) - dem $\bar{N}$ voransteht, oder z.B. das Tempus von $\overline{\mathrm{v}}$ spezifiziert. ${ }^{36}$

Die Kategorien sind nun nicht mehr unterhalb des Lexikoneintrags z.B. als Subkategorisierungsmerkmale - vgl. Standard-Theorie (1965) - angesiedelt, sondern sie werden durch die Revision des Verhältnisses zwischen Merkmal und Kategorie zu einer 
dominierenden Einheit erhoben; d.h. das Kategorienmerkmal hat denselben Status wie jedes andere Merkmal innerhalb des Merkmalkomplexes, der als Bedingung einer Regel beigegeben wird.

\subsection{HALLE, JACKENDOFF, ARONOFF}

Halle (1973) greift einige Vorschläge Chomskys auf und entwirft folgende Wortbildungstheorie: Die Morphologie setzt sich zusammen aus:

(37) (a) einem Lexikon von wurzeln (noots) und Affixen verschiedener Art;

(b) morphologischen Wortbildungsregeln für die Konkatenation von Morphemen, die die worter einer Sprache bilden und - im Gegensatz zu den syntaktischen und phonologischen Regeln global - im Sinne der Standard-Theorie sind;

(c) einem Filter für Ausnahmen (exception bilter).

2u (37(a)): Die Morpheme können nicht nur durch Sequenzen phonetischer segmente repräsentiert werden; sie müssen auch mit grammatischen Informationen versehen werden. $2 u(37(b))$ : Diese Informationen sind dann auch an den lexikalischen Einsetzungsregeln beteiligt, die prätransformational innerhalb der syntaktischen Tiefenstruktur angewandt werden. $\mathrm{Zu}(37(\mathrm{c}))$ : Der Ausnahme-Filter versieht das mittels morphologischer Regeln gebildete Wort mit idiosynkratischen semantischen, syntaktischen und phonologischen Merkmalen und eliminiert die durch morphologische Regeln gebildeten möglichen wörter (potential words). die keine realen, aktuellen wörter der jewelligen sprache sind. Jackendoff (1975) geht von der Behauptung Chomskys (1970) aus, daB Bill's decision to go nicht von Bill decides to go transformational abgeleitet ist, sondern daB decision anhand einer Basis-Regel als eine NP gebildet wird. Das verb decide und das Verbalsubstantiv decision stellen eine Relation dar, die der deskriptiven Adäquatheit der Aktiv-Passiv-Relation Chomskys (bereits 1957) entsprechen. Das Verb decide ist nach Jackendoff mehr zu Basis gehörend als decision; diese Behauptung stutzt er auf die explanatorische Adäquatheit, als Ebene, auf der diese Relation dem AbschätzungsmaB (evaluation measure) unterliegt. 
Mit der Zuordnung der Lexikoneinheit zum AbschätzunsmaB geht Jackendoff von der Definition (9) in Chomsky \& Halle (1968) aus, welche besagt, daB der wert (valuel einer Sequenz von Regeln dem Kehrwert der $\mathrm{zahl}$ von Symbolen in einer minimalen Repräsentation entspricht: $d . h$. je hoher die zahl der symbole in einer Regel ist, um so niedriger ist der Grad der in dieser Regel ausgefuhrten linguistisch signifikanten Generalisierung. ${ }^{37}$ Diese Feststellung veranlabt Jackendoff $(1975,644)$ zu der Behauptung:

of two lexicons describing the same data, that with a lower information content is more highly valued.

Diese Behauptung nennt Jackendoff Full-entry theory evaluation measure.

Die Full-entry Theory wird der Impoverished-entry Theory vorgezogen, obgleich beide der Lexikalisten-Hypothese entsprechen, da die Impoverished-entry Theory 2.B. nur für decide und nicht für decision eine vollspezifizierte Einsetzung (5.642) vorsieht. Die Full-entry Theory răumt decide und decision voll spezifizierte lexikalische Einsetzungen ein, wobei die Redundanzregeln in der Satzbildung - im Gegensatz zu der transformationalistischen und zu der Impoverished-entry Theory - keine Rolle spielen. Die lexikalischen Redundanzregeln dienen auch nicht der Derivation der lexikalischen Einsetzungen, sondern deren Abschatzung (evaluation). Daher werden die lexikalischen Regeln in morphologische Redundanzregeln und in semantische Redundanzregeln (S.650f.) unterteilt.

Die lexikalischen Redundanzregeln spielen auch beim InformationsmaB (information measure) eine Rolle. Das Informationsma besteht darin, daB eine vollspezifizierte lexikalische Einsetzung $w$ gegeben ist, die in das Lexikon eingeführt wird. Dieser lexikalischen Einsetzung wird eine unabhängige Information hinzugefügt, nämlich die Information darüber, das das $w$ im Lexikon existiert. AuBerdem wird der lexikalischen Einsetzung die erlaubte Form (permissive form) hinzugefügt, welche besagt, das jegliche Information beigegeben werden muB, die aufgrund des Vorhandenseins einer morphologischen Regel nicht vorausgesetzt werden kann und durch die das erlaubte $w$ bereits im Lexikon be- 
schrieben ist, einschlieblich anderer lexikalischer Einheiten und semantischer Regeln (S.652).

In der Full-entry Theory haben folglich decide und decision folgende lexikalische Einsetzungen (S.642f.):

(39)

$$
\left[\begin{array}{l}
/ \text { decId/ } \\
+\mathrm{V} \\
+\mathrm{NP}_{1}-\text { on } \mathrm{NP}_{2} \\
\mathrm{NP}_{1} \text { DECIDE ON } \mathrm{NP}_{2}
\end{array}\right]
$$

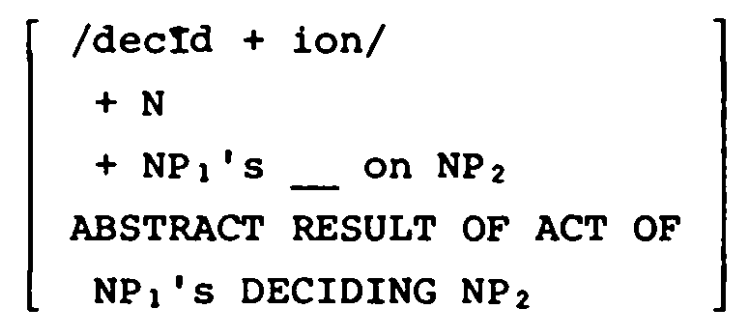

Die lexikalische Einsetzung wird (39) zur Folge durch die phonologische Repräsentation, die syntaktischen Merkmale sowie durch die semantische Repräsentation bestimmt.

Aronoff (1976) geht von einem erweiterten Begriff des Morphems aus. Das Morphem hat für ihn drei Gesichtspunkte: die konstante Form, die willkürliche Verbindung sowie die konstante Bedeutung. Die willkürliche Verbindung bezieht sich auf Sound und Meaning; das englische Morphem mit stellt eine konstante phonetische Kette dar, die willkürlich z.B. mit -ion, -ory oder -or verbunden werden kann. Um mit in die Klasse von Morphemen aufnehmen zu können, muB die konstante Bedeutung erweitert werden, um eine phonologische Operation einzubeziehen. Entscheidend ist nicht so sehr, daB das Morphem etwas bedeutet, sondern vielmehr die Tatsache, daB wir imstande sind, es wiederzuerkennen: es ist also eine phonetische Kette, die an eine andere linguistische Einheit, auBerhalb dieser Kette, angegliedert werden kann. Unter den Worttypen unterscheidet Aronoff zwischen solchen wortern, die aus einzelnen Buchstaben oder Silben zusammengesetzt werden, wie z.B. NATO, Laser, und Wörtern, die aus Morphemen zusammengesetzt werden. Für die erstegenannten worter ist typisch, daB ihre Bedeutung nicht regelmäBig abgeleitet werden kann.

Die Wortbildung stützt Aronoff auf die Hypothese, daB alle regelmäBigen Wortbildungsprozesse auf wortern basieren. Ein neues Wort (S.21f.) wird gebildet, indem man eine reguläre Regel auf ein bereits existierendes Wort anwendet. Beide worter, d.i. das neue und das bereits existierende, sind Mitglieder einer lexikalischen Hauptkategorie. 
Aronoff unterscheidet zwischen wortbildungsregeln und Adjustment-Regeln. Die Adjustment-Regeln werden im output der Wortbildungsregeln angewandt und bilden den Input für die phonologische Komponente. Die Wortbildungsregeln spezifizieren eine Reihe von Wörtern, auf die sie angewandt werden. Diese Reihe oder ein Mitglied dieser Reihe wird zur Basis dieser Regel. Jede Wortbildungsregel spezifiziert eine einheitliche phonologische Operation, die auf die Basis angewandt wird; z.B. die Hinzufügung des Affixes. Jede Wortbildungsregel spezifiziert ausserdem eine syntaktische Ebene nebst Subkategorisierung für das resultierende Wort, sowie die semantische Lesart, die ihrerseits eine Funktion der Lesart der Basis ist. Da nicht alle Morpheme bedeutungstragend sind, werden auch solche Morpheme, die nicht bedeutungstragend sind, in die Wortbildung aufgenommen. Wort und Morphem haben die Eigenschaft als minimale 2 eichen gemeinsam.

Wortbildungsregeln beziehen sich auf die Bildung von wörtern, die im Lexikon vorhanden sind. Jedes Wort im Lexikon sei ein eigener, unabhängiger Eintrag, d.h., daß Wörterbucheintrăge von einander und von den Wortbildungsregeln nicht abhängig sind. Jeder Eintrag entspricht einem vollständigen zeichen. Die Wortbildungsregeln sind von den syntaktischen Regeln getrennt.

\subsection{LASKOWSKI (1981)}

In seiner ausführlichen Diskussion der generativen Wortbildungstheorien, von Chomsky (1970) bis Jackendoff (1975), kommt Laskowski (1977) zum SchluB, daB eine streng lexikalistische Theorie der Wortbildung im Polnischen nicht gerecht werden könnte. Für das Polnische schlägt Laskowski eine transformativ-syntaktische (transformacyjno-syntaktyczna) Wortbildungstheorie vor, die er (1981) folgendermaBen skizziert: Die wortbildung wird allgemein als eine Beschreibung von Regeln verstanden, die neue Lexeme erzeugen und den richtigen Aufbau bereits bestehender Lexeme zeigen. Die Erzeugung neuer Lexeme und die Beschreibung des richtigen Aufbaus bereits bestehender Lexeme sind von einander nicht zu trennen. Jede kreative Wortbildungsregel dient nicht nur der Erweiterung des Lexikons der jeweiligen Sprache, sondern beschreibt gleichzeitig die innere struktur der Lexeme, 
die durch diese Regel erzeugt werden; darin liegt auch das Verhältnis zwischen dem Lexem als einem produkt der derivativen Operation (der fundierten Einheit) und dem Lexem als dem Ausgangspunkt dieser Operation (der fundierenden Einheit) begründet. Ebenso kann von keiner unproduktiven Regel behauptet werden, sie sei völlig unkreativ (S.109f.); letztere Behauptung stützt Laskowski auf die Tatsache, daB bestimmte passive Wortbildungsregeln unter bestimmten Umständen - in diachronischer Sicht - produktiv würden. Wird eine passive, unproduktive Regel produktiv, so handelt es sich nicht um die Erweiterung der Grammatik durch eine neue Regel, sondern um die Aktualisierung des Analogie-Mechanismus, der auf einer in Lexikon vorhandenen Regel beruht, welche die Relationen zwischen den Lexikoneinheiten bezeichnet. 38

Die Wortbildungsregeln - sowohl die produktiven als auch die unproduktiven - haben stets lexikalischen Charakter. Sie bilden Verallgemeinerungen in sinne funktionaler und formalen Relationen, die zwischen realen Elementen des jeweiligen Sprachsystems bestehen. Dabei ist der kreative Charakter der Wortbildungsregel gegenüber ihrem passiven, relationalen charakter sekundär.

Geht man davon aus, daß die lexikalischen Einsetzungsregeln (reguzy doboru jednostek szownikowych) im Sprachgefühl des idealisierten Sprecher-Horrers durch die Beziehung zwischen dem semantischen Komplex und den phonologischen Repräsentationen bestimmt sind, dann herrscht zwischen dem bestimnten Typus der semantischen struktur und der bestimmten formalen (morphologischen) struktur Assoziation.

Die Annahme des lexikalischen Charakters der Wortbildungsregeln entscheidet für Laskowski keineswegs die Kontroverse zwischen der lexikalistischen und der transformationalistischen Wortbildungstheorie. Das Wesentliche dieser Kontroverse ist seiner Meinung nach nicht der lexikalistische oder der syntaktische Status der Wortbildungsregeln, sondern die Metasprache, die die Relationen in der Wortbildung (S.112f.) beschreibt. In diesem Zusammenhang wendet sich Laskowski gegen die Ad-hoc-Behauptung von Aronoff - vgl. $\S 5.2$-, das die Wortbildungsregeln von sämtlichen anderen grammatischen Regeln getrennt seien und daB 
sich die Wortbildungsregel auf die syntaktischen, semantischen und phonologischen Regeln beziehen solle. Weder anhand der Theorie von Halle (1973), noch der von Jackendoff (1975) und von Aronoff (1976) können Derivate erklärt werden, die z.B. Ergebnisse von univerbierungen sind, wie z.B. dwurzedowka - marynar$k a$ dwurzedowa 'Zweireiher (Sakko)' oder kostiumbwka - proba kostiumowa 'Kostümprobe'. Die Tatsache, daB die genannten Wortbildungstheorien mit dem Problem der idiosynkratischen Eigenschaften der Lexeme fertiggeworden sind, geschah auf Kosten der Möglichkeit, die Beziehungen, die zweifelsohne zwischen den Wortbildungs- und den syntaktischen Relationen bestehen, aufzeigen zu können.

Um u.a. die Univerbierung im Polnischen zu beschreiben (S.114f.), bedarf es einer transformationalen Regel, die die Beziehungen zwischen einer syntaktisch erzeugten nominalen Gruppe und dem morphologisch erzeugten univerbierten Lexem zeigt. Die Eingangsregel ist:

(40) $\left[\left[\left[\begin{array}{c}Y \\ \alpha \text { GENUS } \\ (\beta \text { NUMERUS })\end{array}\right]\right]_{N}[X]_{A}\right]_{N G} \rightarrow\left[\begin{array}{c}{[X]_{A}+[+ \text { SUFFIX }]} \\ \alpha \text { GENUS } \\ (\beta \text { NUMERUS })\end{array}\right]_{N}$

In (40) wird die nominale Gruppe (NG), bestehend aus $N$ und $A$, durch $N$ ersetzt, wobei von den Merkmalen, die $N$ kennzeichnen, allein $Y$, also die phonologische Repräsentation getilgt wird; Genus und Numerus bleiben erhalten. Die Tilgung von $Y$ hat die Hinzufügung des Merkmals [+ SUFFIX] und die gleichzeitige Ersetzung der NG durch $\mathrm{N}$ zur Folge. Während die Univerbierungstransformation von der syntaktischen struktur der NG abhängig ist, wird die wahl der morphonemischen Repräsentation des Suffixes $u . a$. durch Genus und Numerus des $N$ innerhalb der NG sowie durch die morphonemische struktur des suffigierten Teils der univerbierten $\mathrm{N}$ bedingt. Für die morphonemische Repräsentation des suffixes nimmt Laskowski folgende Regel, die als Ergănzung von (40) gesehen werden muß, an ${ }^{39}$ :

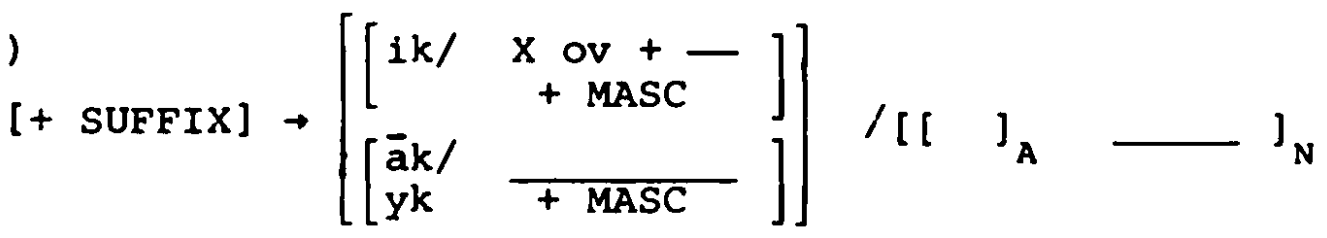


Unter den drei Fypen der Wortbildungsregeln, nämlich den Derivationsregeln, den Affigierungsregeln und den Adaptionsregeln - vgl. Aronoffs Adjustment-Rules - nehmen die Derivationsregeln eine Schlüsselposition ein; die Derivationsregeln bestimmen die für die jeweilige sprache charakteristischen funktionalen Relationen zwischen den Elementen des Lexikons. Die Affigierungsregeln werden von den Derivationsregeln obligatorisch impliziert. Die Adaptionsregeln sind fakultativ.

Die Derivationsregeln bestimmen nach Laskowski den Status der wortbildungstheorie, deren transformationalen oder nichttransformationalen Charakter. Ohne ins Detail zu gehen, behauptet Laskowski (S.116), daB zumindest einige unter den Derivationsregeln transformationalen Charakter haben. Was die Affigierungsregein betrifft, so entscheidet weder eine transformationalistische noch eine lexikalistische Konzeption über deren Gestalt und deren Funktionieren. Im Input der Affigierungsregeln stehen Symbolketten, die mittels Derivationsregeln erzeugt sind; dabei ist es für die Anwendung der Affigierungsregeln gleich, ob diese symbolketten transformational oder lexikalisch erzeugt sind. Wesentlich ist, daB für eine adäquate zuordnung von Affigierungsregeln den jeweiligen Derivationsregeln sowohl die Inputbedingungen als auch die outputbeschränkungen berücksichtigt werden müssen. Die Inputbedingungen entsprechen den kontextuellen Bedingungen für die Verbindung der jeweiligen Wortbildungsaffixe und der lexikalischen Morpheme. Die outputbeschränkungen entsprechen der Notwendigkeit phonotaktischer Beschränkungen und insbesondere der Notwendigkeit der Beachtung des Grundsatzes der wiedenerkennbarkeit der wortbildungsgrundlage.

Die Inputbedingungen der Affigierungsregeln können die semantischen, syntaktischen, morphologischen und phonologischen Merkmale der Formative betreffen, mit denen die Derivationsregeln operieren; sie können die syntaktische struktur der Reihen betreffen, auf die Regeln der Univerbierung oder der Derivation, die von pronominalen Gruppen ausgeht, angewandt werden. Nach Laskowskis Meinung widerspricht die depronominale Derivation der Behauptung Aronoffs, daB alle regulären Wortbildungsprozesse auf dem Wort - vgl. $§ 5.2$ - basieren. Als völlig "falsch" beurteilt Laskowski die Behauptung Aronoffs $(1976,21)$, daB das 
neue (=erzeugte) Wort und das bereits existierende den lexikaIischen Hauptkategorien angehören. Uberhaupt wendet sich Laskowski gegen die Behauptung, daB die Grundlage der Derivation nur Lexeme sein können, die zu den lexikalischen Hauptkategorien gehoren; selbst dann, wenn Derivate wie z.B. syczed 'z1schen' oder miauczed 'miauen' unbeachtet blieben, sollten Derivate wie z.B. potakiwac 'bejahen' brücksichtigt werden.

\subsection{TEILAUSWERTUNG}

Die Auswahl der in $\S 5.1-\S 5.3$ referierten Wortbildungstheorien geschah bereits im Hinblick darauf, das die sog. lexikalistische Methode als Orientierungsstutze für die Untersuchung der Nominalisierung im Polnischen aufgefabt wird. Dies soll unter Einbezlehung der Bedenken von Laskowski - in $\S 5.3$ - geschehen, die, wie es die Untersuchung zeigen wird, nur teilweise berechtigt sind, jedoch keineswegs den Rahmen der syntaktisch - im Gegensatz zu semantisch (vgl. \$4.0 - \$4.2) - orientierten Grammatik in Frage stellen. Dabei soll der standort der Wortbildung innerhalb der S-Basis - wie er in $\S 3.1$ festgelegt worden ist - gewahrt bleiben.

Die folgenden zwel Paragraphen, nămlich $\S 5.4 .1$ sowie $\S 5.4 .2$, sollen im Rahmen einer Teilauswertung der hier referierten Methoden einige Grundprobleme aufzeigen, die der Nominalisierung in Polnischen voranzuschicken sind.

\subsubsection{PARTIZIP UND DEVERBALES NOMEN}

Das zugestăndnis, das Chomsky (1970) an die sog. Transformationalisten - vgl. $\S 5.1$ - macht, mus auch bel der Bildung des Partizips im Polnischen gemacht werden. Das polnische adjektivische participium praesentis activi wird in der Regel durch einen Relativsatz ersetzt, namlich:

$$
\mathbf{N}-\mathbf{P R O}-\mathbf{V} \rightarrow \mathbf{N}-\text { PART }
$$

so z.B. in:

(43)(1) czlowiek, co pracuje

'(der/ein) Mensch, der arbeitet'

(11) czlowiek pracujqcy

'(der/ein) arbeitende(r) Mensch' 
In (43(i)) und (43(ii)) kann czzowiek durch das Demonstrativpronomen ten 'dieser' oder durch das Personalpronomen ktob 'jemand', das Relativpronomen co dagegen auch durch ktory 'welcher' ersetzt werden, ohne daB sich die Beziehung zwischen Relativsatz und Partizip verändert. Die Ausgangsbasis in (43) ist (30) entsprechend:

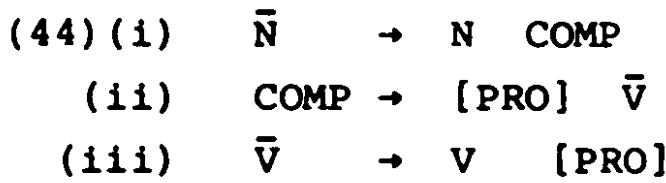

In (44) werden zwei PRO-Elemente eingesetzt; das eine steht links von $\bar{v}$ und wird entweder durch das Relativpronomen oder durch e (= empty) ersetzt, das zweite PRO-Element steht rechts von V. Wird das erste PRO-Element in (44(11)) durch e gekennzeichnet, so kann das zweite PRO-Element in $(44(111))$ durch das partizipiale -qc-ersetzt werden. Ist das zweite PRO-Element durch e gekennzeichnet, so kann das erste wiederum durch das Relativpronomen ersetzt werden. Das $N$, links von COMP (= Complementiser), bestimmt das Genus, hier Maskulinum, das zwischen $\mathrm{N}$ und COMP eingesetzt wird.

Die Ersetzungen in (44) entsprechen im Polnischen folgender Struktur, die mittels indizierter klammern dargestellt wird:

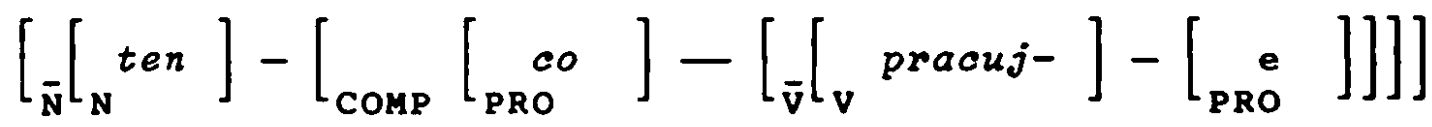

Die Struktur (45) entspricht also $(43(1))$. Soll nun das partizip abgeleitet werden, so wird das erste PRO-Element durch $e$, das zweite PRO-Element dagegen durch -qc- ersetzt.

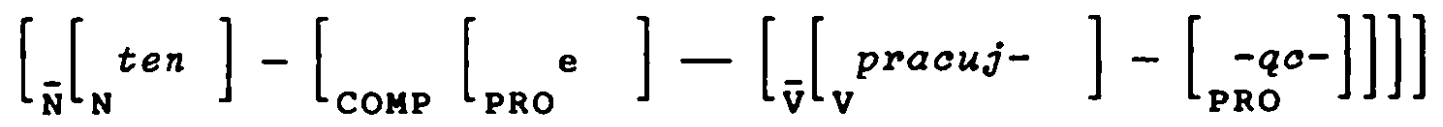

Als V-Repräsentation kann sowohl in (45) als auch in (46) nur von der flektierten Form ausgegangen werden; vgl. Infinitiv pracowac: sonst hiebe es nämlich *pracowqc-y. Das bedeutet aber, daß die Partizipialisierung im Polnischen ebenfalls nicht in der Satz-Basis, sondern in der selchtstruktur stattfindet; es kann also erst partizipialisiert werden, wenn die Flexionsendung, hier reprăsentiert durch die morphonemische Entsprechung von $-u j-$, realisiert worden ist. 
Für die Partizipialisierung im Polnischen gilt generell folgende Basis-struktur:

$(43.1)$

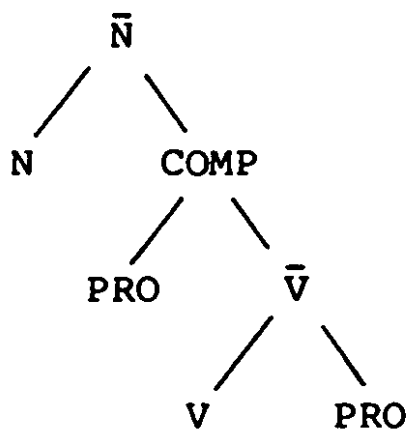

(43.1) entspricht den Ersetzungen (44) sowie den Einsetzungen in (45) und $(46)$.

Der Relativsatz in (43(i)) gilt samt Nomen bzw. Pronomen des Hauptsatzes auch als Paraphrase - vgl. DOR - für das Nomen agentis pracownik 'Arbeiter'. Hinzu kommt, daB (43(ii)), also czlowiek pracujqcy 'der/ein arbeitende( $r$ ) Mensch' ebenfalls durch pracownik ersetzt werden kann; wobei als Denotatum das ADV zawodowo 'beruflich' hinzugefügt werden kann. Demnach müBte (43.1) auch für die Bildung des Nomen agentis gelten.

Der Annahme, daB die Wortbildung in der Satz-Basis - und nicht in der Seichtstruktur - stattfindet und daB diese Annahme keine bloße Behauptung ist, trägt das Polnische insofern Rechnung, als es - im Gegensatz zur Partizipalisierung (vgl. S.59) - nicht die finite bzw. flektierte Form des worts einsetzt, sondern die infinite bzw. personunmarkierte. So gilt auch für den $\mathrm{V}$-stamm in prac-ow-nik das infinite pracowa-d und nicht - wie beim Partizip - pracuj-e '(1.PERS. SG. PRÄS.)'.

Der Relation zwischen Dominanz und Präzedenz - vgl. $\S 3.1$ - entsprechend, sowie der Behauptung, daB das Wort, bzw. der lexikalisch kategorisierte wortstamm in den terminalen Knoten eingesetzt wird, kann im Falle von pracownik kein comp rechts von $N$ - vgl. (43.1) - angenommen werden. AuBerdem wäre es auch nicht begründbar, solch ein Merkmal, dem ja der verbale Wortstamm zugeordnet wird, um ihn mit dem nominalen suffix - nik 2 u verbinden, von $\overline{\mathbf{N}}$ und nicht von $\mathbf{N}$ dominieren $z$ u lassen; also muB das Nomen agentis pracownik bereits in der satz-Basis in den terminalen Knoten $\mathrm{N}$ eingesetzt werden.

Im Gegensatz zur Partizipialisierung gilt - entsprechend (15) - 
- für die Nominalisierung im Polnischen generell die Ersetzung des terminalen Knotens $\mathrm{N}$ :

$(47.0)$

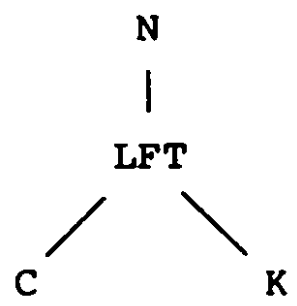

Demnach markieren $C$ und $K$, beide von LFT dominiert, in der Anordnung $C$ präzediert $K$ das in den terminalen Knoten $N$ einzusetzende Wort. Im Falle von pracownik wird der V-Stamm pracois.r- in $C$, das suffix -nik dagegen in $\mathrm{K}$ eingesetzt.

Die Struktur (47.0) gibt folgende Möglichkeit, die Paraphrasc dieses Nomen agentis einzubeziehen:

$(47.1)$

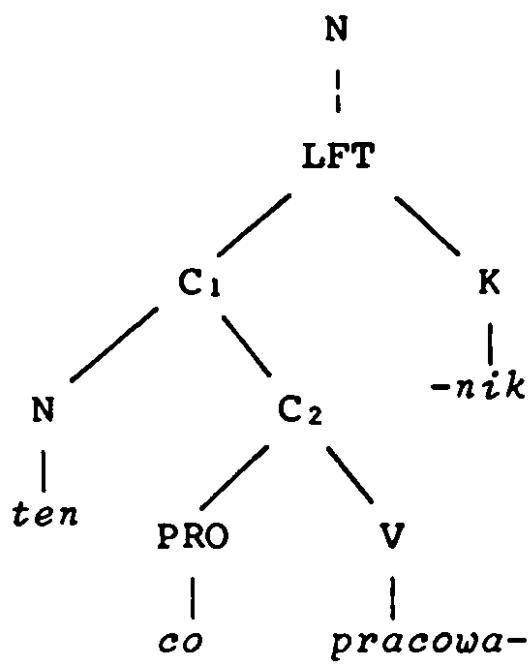

Die Voraussetzung für (47.1) ist die Annahme - entsprechend Chomsky $(1981,55 \mathrm{ff.})$ - der Kategorie $t$ (trace), die die Möglichkeit gibt, in der Paraphrase eine Spun hinter das Verb zu setzen, die die Merkmale von ten bzw. czzowiek vereint, nämlich:

(47.2) czlowiek i co pracowa- $t_{i}$

Da weder czZowiek noch ten die Kategorie $t_{i}$ als Wort, d.h. morphonemisch repräsentiert ersetzen können, dafür aber als Merkmalkomplex aus [+ INDIVIDUATIVUM; + BELEBT; + MENSCHLICH], und da dieser Merkmalkomplex der zuordnung des suffixes - $n i k$ entspricht, das von $\mathbf{N}$ dominiert wird, tritt anstelle der spur $t_{i}$ das Suffix, bzw. die K-Repräsentation von -nik; dies selbstverständlich dann, wenn PRO in (47.1) durch e ersetzt wird. 
Die Kategorie $t$ wird in (47.2) also nicht als satzsyntaktische, sondern als morphosyntaktische Kategorie eingesetzt. Als morphosyntaktische Kategorie wird sie ebensowenig morphonemisch repräsentiert wie als satzsyntaktische Kategorle. In ihrer Funktion als morphosyntaktische Kategorie vereint sie sämtliche Merkmale, die für die zuordnung der jeweiligen K-Repräsentation zum Wortstamm entscheidend sind, $d . h$. in (47.1) vereint t sämtliche Merkmale, die den markierten Teil, d.1. die von $C$ daminierten und $V$ präzedierenden Knoten widerspiegeln. Die ebenfalls morphosyntaktische Kategorie PRO ordnet in (47.1) die Merkmale des markierten Teils dem Merkmal des unmarkierten Teils, nămlich dem Merkmal des v-Stamms zu.

C präzediert in (47.1) keine lexikalische, sondern ebenfalls eine morphosyntaktische Kategorie, nämlich $K_{\text {; }}$ wăhrend beide, d.1. $C$ und $K$ von der lexikalischen Kategorie $N$ dominiert werden. Der Merkmalkomplex von czZowiek kann also deshalb in $t$ eingesetzt werden, weil $K$ ebenfalls von $N$ dominiert wird; nur wird $\mathrm{K}$ im Gegensatz zu dem Knoten, in den czlowiek eingesetzt wird, vom terminalen Knoten $N$ regiert; vgl. dazu FuBn. 42. Aufgrund der Dominanz, die das Regieren - in (47.1) rechte Se1te - einerseits, und die Prăzedenz - linke Seite - zur Folge hat, kann festgestellt werden, daB in der Wortbildung nur den regierten terminalen Knoten des LFT eine morphonemische Repräsentation zugeordnet wird, nämlich - in (47.1) - dem unmarkierten knoten $\mathbf{V}$ sowie dem knoten $k$. Der knoten $V$ wird von $C$ regiert, der knoten $K$ wird von $N$ bzw. LFT reglert.

Die regierten terminalen Knoten bestimmen auch die Indizierung der innerhalb der Satz-Basis einzusetzenden wortbildungsregel. Nomina agentis, wie z.B. pracownik, werden gemă folgender Wortbildungsregel gebildet:

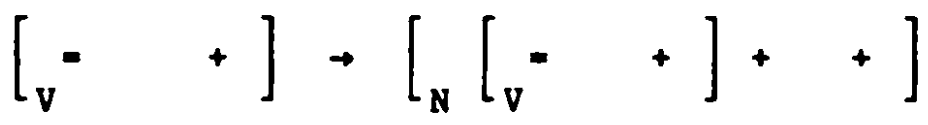

$$
\{++\}_{N} \rightarrow\{+h i k+\}_{N}
$$

Regel (47.3(1)), d.1. die Derivationsregel, stellt die Bildung sămtlicher deverbaler Nomina in Polnischen dar, wăhrend 147.3 (11)), d.1. die Affigierungsregel, die morphonemische Repräsen- 
tation von $\mathrm{K}$ bestimnt. Beide Regeln werden in der Satz-Basis eingesetzt. Die Derivationsregel (47.3(i)) wird also auf die regierten knoten in (47.1) beschränkt, $d . i$. auf $V$ und $N$; dabei entspricht $V$ dem unmarkierten Teil des LFT, $N$ dagegen, da die von ihm regierte $\mathrm{K}$-Repräsentation die Merkmale von $t$ vereint, dem markierten Teil des LFT. Demnach gilt die Affigierungsregel (47.3(ii)) der morphonemischen Repräsentation des markierten Teils des LFT.

Doch wie sieht es mit anders strukturierten Paraphrasen aus, so z.B. mit:

(47.4) (i) ten, co handluje kwiatami - kwiaciarz 'der, welcher mit Blumen handelt - Blumenhändler'

(ii) ten, co pracuje naukowo - naukowiec 'der, welcher wissenschaftlich arbeitet - wissenschaftler'

(iii) fabryka, gdzie produkuje sie papier - papiernia 'Pabrik, in der Papier hergestellt wird - Papierfabrik'

Entsprechend (47.0) können die Paraphrasen folgendermaBen eingesetzt werden:

$(47.4 .1)$

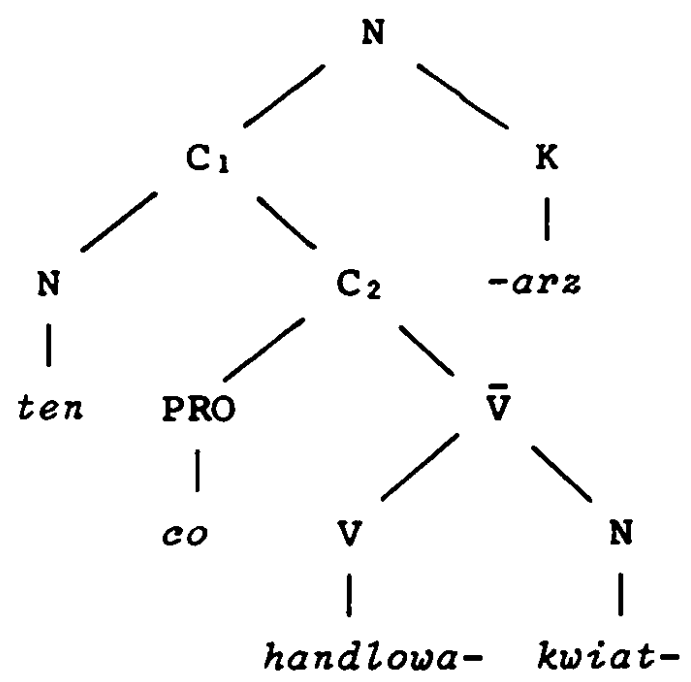

Die Struktur (47.4.1) zeigt, daB auch bei der Bildung des Nomen agentis kwiaciarz nur die regierten terminalen Knoten des LFT morphonemisch zum Ausdruck - im wortwörtlichen sinne - konmen, während das präzedierende $N$ durch die Merkmale 【+ BELEBT; + MENSCHLICH], das präzedierende $V$ durch [+ TÄTIGREIT] und PRO als verbindendes Element der Präzedenzen morphosyntaktisch durch e realisiert werden.

Für die Bildung des Nomen agentis in (47.4(ii)) kann folgende Einsetzung vorgenommen werden: 
(47.4.2)

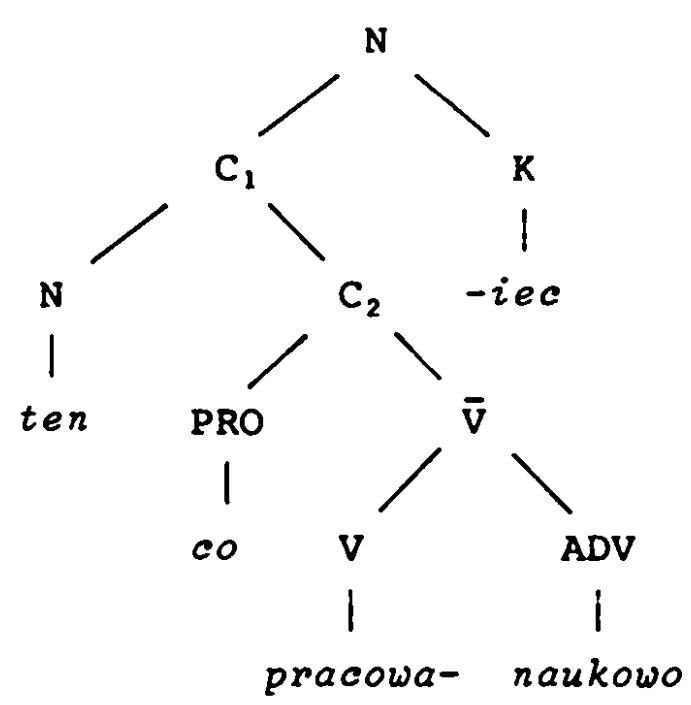

Im (47.4.2) gelten die gleichen Merkmale wie bei der Bildung von kwiaciarz in (47.4.1); den Unterschied stellt allein das regierte $A D V$ dar, das den adjektivischen wortstamm repräsentiert. Während das von $\mathrm{N}$ regierte $\mathrm{K}$ durch $-i e c$ graphemisch repräsentiert wird; dies in naukowiec.

In den terminalen Knoten ADV wird deshalb der A-Stamm eingesetzt, weil auch im Polnischen das ADV erst vom A gebildet werden muß, und dies ebenfalls durch eine gesonderte Regel; zum englischen ADV siehe: Radford $(1981,133)$.

Für (47.4(ii1)) gilt folgende Einsetzung:

$(47.4 .3)$

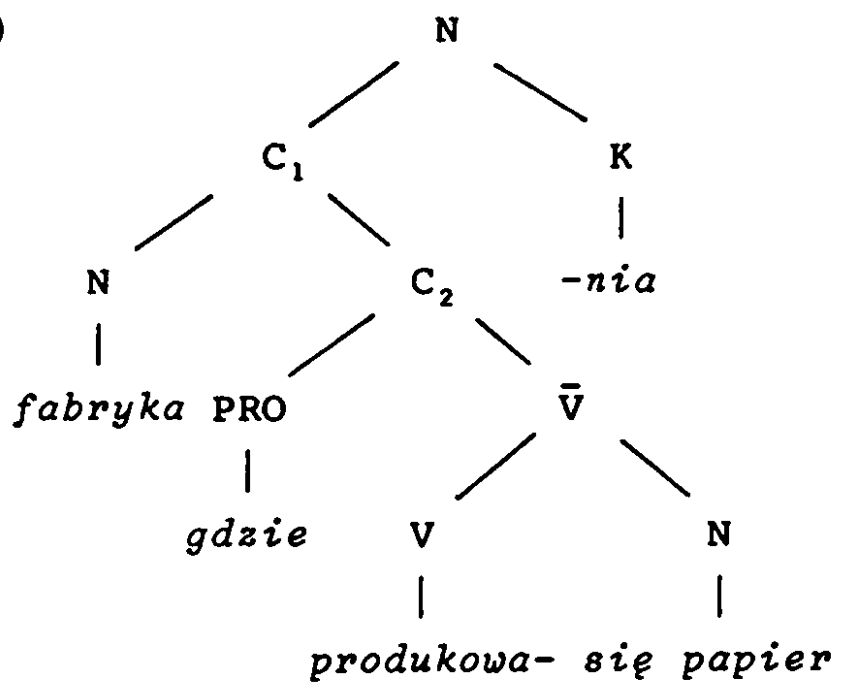

Im Gegensatz zu den anderen Beispielen gelten hier die Merkmale [+ INDIVIDUATIVUM; - BELEBT; - ABSTRAKT]. Hinzu kommen die Merkmale [+ LOKAL], bedingt durch PRO, sowie [+ TÄTIGKEIT] entsprechend V. Das Nomen papiernia wird ebenfalls von den regierten terminalen Knoten gebildet. 
Die hier eingesetzten Paraphrasen unterscheiden sich von denen, die dem Partizip zugrundegelegt werden, durch ihre stellung in der Satz-Basis. Wăhrend die Paraphrase $(43(1))$ im Hinblick auf die Partizipialisierung von $\overline{\mathrm{N}}$ dominiert wird - vgl. (46) und (47) - , so wird sie bei der Nominalisierung von $\mathrm{N}$ bzw. C dominiert; m.a.W.: Soll ein Partizip gebildet werden, so wird die ihm zugrunde liegende Paraphrase bzw. Wortgruppe in die Phrasen-struktur der Satz-Basis eingesetzt, um nach der Realisierung der finiten bzw. flektierten Form innerhalb der seichtstruktur die Partizipendung hinzuzufügen, soll dagegen ein Nomen gebildet werden, so wird die paraphrase bzw. die Wortgruppe, welche ihm zugrundegelegt werden kann, in den vom terminalen Knoten dominierten lexikalischen Formationsteil LFT eingesetzt, wobei der markierte Teil der Paraphrase bzw. Wortgruppe den Merkmalen entspricht, die für die zuordnung der entsprechenden nominalen $\mathrm{K}$-Repräsentation an den unmarkierten, morphonemisch repräsentierten Wortstamm von Bedeutung sind.

\subsubsection{UNIVERBIERUNG}

Die von Laskowski (1981) aufgezeigte Univerbierung - vgl. \$5.3- setzt die Berücksichtigung der stellung des Adjektivs bezüglich des Nomen im Polnischen voraus.

Im Englischen - vgl. Radford $(1981, \S 3)$ - wird die AP durchweg links von $\overline{\mathrm{N}}$ eingesetzt, nämlich:

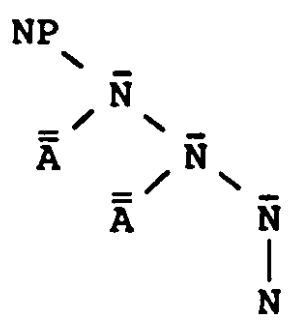

Grundsätzlich gilt auch diese stellung des Adjektivs für das polnische, so z.B. in: mqdry czzowiek 'kluger Mensch', biaze sanie 'weiBer Schlitten', okragzy stoz 'runder Tisch'. Es handelt sich also um die stellung des Adjektivs $v$ or dem Nomen, mit dem es das Genus gemeinsam hat.

Neben dem vorangestellten Adjektiv gibt es Adjektive, die hinter dem Nomen stehen, so z.B. In zodz zaglowa 'segelboot' , otatek zaglowy 'Segelschiff', kolej wqokotorowa 'Schmalspurbahn' 
u.v.a. Diese Wortverbindungen bzw. Wortgruppen - Laskowski nennt sie nominale Gruppen - werden univerbiert zu zaglowka, zaglowiec, wqskotorowka mit derselben Bedeutung wie die ihnen zugrunde liegenden Wortgruppen. Syntaktisch gesehen müBte angesichts der stellung des Adjektivs hinter dem Nomen fürs Polnische eine Ausnahme gemacht werden. ${ }^{41}$ Diese Ausnahme bestünde darin, dab für das Polnische nicht nur die stellung (48), sondern zusätzlich eine zweite, rechts von $\mathrm{N}$ angenommen werden müBte; denn es kann ebenso heiBen biaza zodź zaglowa 'weiBes Segelboot' mit der wortstellung $A$ vor $\mathbf{N}$ vor $A$.

Vergleicht man biaza zodź mit zodź zaglowa, also 'weiBes Boot' mit 'Segelboot' mit der Wortstellung $A$ vor $N$ vs. $N$ vor $A$, so kann man feststellen, daB die A-Repräsentation in ersten Fall, also in $A$ vor $N$, austauschbar ist: denn es kann ebenso heiBen piekkna Zodź 'schönes Boot' oder wqska Z6dz' 'schmales Boot'. Im zweiten Fall, also in $\mathrm{N}$ vor $\mathrm{A}$, dagegen ist nicht die A-, sondern die $N$-Repräsentation gegen statek 'Schiff' austauschbar, dies in dem bereits zitierten statek zaglowy 'Segelschiff'. In beiden Fällen wird also das Nomen von $\mathbf{N}$ regiert, während im ersten Fall A die N-Repräsentation präzediert; dies innerhalb der Phrasenstruktur (48). 42

Für die Wortstellung $\mathrm{N}$ vor $\mathrm{A}$ bedarf es einer anderen Einsetzung. Diese andere Einsetzung wird dadurch erforderlich, daB die $\mathrm{N}-\mathrm{A}-\mathrm{Verbindungen}$ eine einzige Bedeutungseinheit bilden; diese in dem Sinne, daB 'Boot' oder 'Schiff' bezüglich eines für sie charakteristischen Teils, nämlich des zur Fortbewegung dienenden Teils benannt sind. Da also zodz bzw. statek und zaglow-a/-y jeweils als eine Bedeutungseinheit zusammengehören, ist auch deren Univerbierung möglich; dies im Gegensatz zu den $\mathrm{A}-\mathrm{N}$-Verbindungen. ${ }^{43}$

Syntaktisch bzw. morphosyntaktisch gesehen enstpricht diese Bedeutungseinheit, die sowohl als N-A-Verbindung als auch als deadjektivisches Nomen ausgedrückt werden kann, der Einsetzung in n u $r$ einen terminalen Knoten; im Falle solcher wörter wie zaglowka, zaglowiec und wqskotorowka ist es $\mathrm{N}$. Doch welcher Knoten ist es im Falle der Entsprechungen Zodź zaglowa, statek zaglowy, kolej wqskotorowa? 
Aufgrund der Einsetzungen (47.1) sowie (47.4.1)-(47.4.3) kann festgestellt werden, daß der terminale knoten $\mathrm{N}$ in allen Fällen das nominale suffix regiert, d.h., daß das einzusetzende Wort die lexikalische Kategorie $\mathrm{N}$ haben muB. Wird nun das univerbierte Nomen iaglowka eingesetzt, so entspricht - $k$ - der von $\mathrm{N}$ regierten nominalen $\mathrm{K}$-Repräsentation. In den genannten Einsetsetzungen kann aber auch festgestellt werden, daß die den unmarkierten Wortstamm präzedierenden Wörter die inhärenten Merkmale widerspiegeln, die für die zuordnung des entsprechenden nominalen Suffixes von Bedeutung sind. Es kann also davon ausgegangen werden, daß bei der Bildung des Nomen agentis raukowiec 'Wissenschaftler' auf die morphonemische Repräsentation des markierten, da den adjektivischen wortstamm präzedierenden Teils des LFT ebenso verzichtet werden kann, wie dies bei der Ersetzung von Zodz zaglowa durch zaglowka der Fall ist: denn an der Bedeutung ändert sich dabei nichts.

Da sowohl für Zodí iaglowa als auch für $\dot{z} a g l o w k a$ die gemeinsamen Merkmale u.a. [+ INDIVIDUATIVUM; - BELEBT; + FORTBEWEGUNGSMITTEL] gelten und da sich beide Ausdrücke nur darin unterscheiden, daB in der $\mathrm{N}-\mathrm{A}$-Verbindung der markierte Teil obendrein noch morphonemisch zum Ausdruck gebracht wird, kann für beide Ausdrücke folgende Einsetzung angenommen werden:

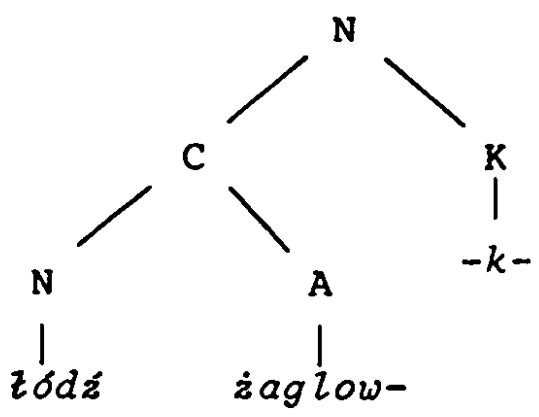

Wird hier auch die Kategorie $t$ in ihrer morphosyntaktischen - vgl. $\$ 5.4 .1$ - Funktion eingesetzt, so gilt:

(49.1) zodz $z_{i}$ zaglow- $t_{i}$

Wird also das markierte Nomen $z \delta d z$ in (49.1) nach $t$ bewegt, so wird infolge dieser Bewegung die nominale $\mathrm{K}$-Repräsentation - $k-$ in (49) realisiert, wăhrend gleichzeitig zodź seine morphonemische Repräsentation verliert. Bleibt zodz in seiner Position stehen, so wird $\mathrm{K}$ morphonemisch nicht reprăsentiert. 
Die Tatsache, das bei dieser Bewegung, die der Univerbierung zugrunde gelegt wird, allein die Merkmale bewegt werden - die Kategorie $t$ kann, wie bereits in $\S 5.4 .1$ festgestellt wird, durch keine morphonemische Repräsentation ersetzt werden - kann besonders durch solche wörter verdeutlicht werden, die zwar Ergebnis solch einer Univerbierung sind, jedoch mit der $\mathrm{N}-\mathrm{A}-\mathrm{Ver}-$ bindung, die ihnen innerhalb der aktuellen sprache zugrundeliegt, nicht mehr übereinstimmen, so z.B. ciziarowka (FEM) - samochod cifzarowy (MAS) 'Lastkraftwagen' oder ogolniak (MAS) - Liceum ogolnoksatazcqce (NEU) 'Allgemeinbildendes Lyzeum'; dabei ist die Nichtubereinstimmung im Genus hier das ausschlaggebende Argument; denn zur Reversibilität gehört nach Laskowski - vgl. Algorithmus (40) - die Ubereinstimmung im Genus. Andere Univerbierungen lassen sich nur noch allein auf ein abstraktes, u.a. bezüglich Genus markiertes $\mathbf{N}$ zurückführen, so 2.B. stalowka - N (FEM) stalowa 'Stahl-(Schreib)feder' oder placowka - N (FEM) placowa 'Feldwache, Niederlassung'. In diesen Univerbierungen konzentrieren sich die inhärenten Merkmale, die sonst durch ein präzedierendes Nomen morphonemisch repräsentiert werden können, assoziativ allein auf die K-Repräsentation der Univerbierung.

Die Univerbierungen - im Indeks a tergo $\mathrm{zu}$ DOR sind es rund 800 allein auf - $6 w k-a$ - lassen darauf schließen, daß im Polnischen die Tendenz vorherrscht, nicht die $\mathrm{N}-\mathrm{A}$-Verbindungen, sondern die Univerbierungen einzusetzen; wer sagt schon kiezbaska parowa statt parowka 'Brühwurst'. Damit trägt diese Tendenz der Feststellung Rechnung, daB nur das unmarkierte Element an der morphonemischen Repräsentation des Wortstamms teilhat. Die Tatsache, daB auf die markierte $\mathrm{N}$-Repräsentation verzichtet werden kann, ist auf die Selbständigkeit der K-Repräsentation im Zusammenhang mit der unmarkierten A-Repräsentation zurückzuführen; dies im Hinblick auf die Assoziation mit den entsprechenden Merkmalen und damit mit dem bezeichneten objekt, das - ebensowenig wie in pracownik (vgl. (47.1)) - morphonemisch nicht repräsentlert $z u$ sein braucht. Es genügt, wenn statt dessen seine Merkmale der Affiglerungsregel als Bedingungen beigegeben werden.

Was die Wortbildungsregeln betrifft, so könnte man von zweier- 
lei Behauptungen ausgehen: a) zaglowka wird von zodź zaglowa gebildet; b) zaglowka und zodź zaglowa haben zwar die gleiche Bedeutung, jedoch zwei getrennt von einander zu bildende morphosyntaktische strukturen.

Die Behauptung (a) setzt das Vorwegschicken eines Algorithmus voraus, der auf die Reversibilität von zodź zaglowa vs. zaglow$k a$ hinweisen würde. Solch einem Algorithmus entspricht bereits (40) von Laskowski. Nähme man solch einen Algorithmus an, so mübte konsequenterweise auch für Nominalisierungen vom Typ der genannten Nomina agentis, die ebenfalls durch Wortgruppen $z u$ ersetzen sind und die ebenfalls in der Bedeutung übereinstimmen, entsprechende Algorithmen angenommen werden. Diese Algorithmen müBten den jeweiligen wortbildungsregeln und jeweiligen Affigierungsregeln vorweggeschickt werden. Solch eine Vorgehensweise würde jedoch den WortbildungsprozeB unnötig komplizieren. Algorithmen dieser Art wären allzu sehr an der Bildung von Paraphrasen innerhalb der Satzsyntax orientiert, die, gemessen an der hier dargestellten Wortbildung einen vorllig getrennten ProzeB darstellt. Ferner wäre solch ein Algorithmus, der die Reversibilität $\mathrm{zwischen} \mathrm{N}-\mathrm{A}$-Verbindung und Univerbierung zeigt, spätestens dann nutzlos, wenn die genannten Nichtubereinstimmungen im Genus oder das Fehlen der morphonemischen Repräsentation des markierten Nomen eintreten.

Die Behauptung (b) hingegen gibt die Möglichkeit, völilig unabhängig von vorweganzuwendenden Algorithmen, für beide morphosyntaktische strukturen jeweils eine wortbildungsregel aufzustellen; dies entsprechend der Feststellung, daB Zodź zaglowa und zaglowka zwar die gleiche Bedeutung, jedoch unterschiedliche morphosyntaktische strukturen haben.

Für die Bildung des sog. univerbierten Nomen, wie z.B. zaglow$k a, g i l t$ also folgende Wortbildungsregel:

(50.1) $\left.\left[{ }_{A}\right] \rightarrow\left[{ }_{N}\right]++\right]$

Anhand entsprechender Affigierungsregeln, in welchen entsprechende Merkmale als Bedingungen für die morphonemische Realisierung der $\mathrm{K}$-Repräsentation stehen, wird das Suffix $\{+\ldots+\}$ entsprechend morphonemisch realisiert. 
00047501

70

Für die Bildung der N-A-Verbindung, wie z.B. Zodź żaglowa, gilt folgende wortbildungsregel:

(50.2) $\left[_{\mathrm{N}}\right]-[\mathrm{A}] \rightarrow\left[\left[_{\mathrm{N}}\right]-\left[\begin{array}{l}\mathrm{A} \\ \end{array}\right]\right.$

Für die Einsetzung sowohl der Univerbierung als auch der $\mathrm{N}-\mathrm{A}-$ -Verbindung gilt (49).

Alek Pohl - 9783954795123

Downloaded from PubFactory at 01/10/2019 04:55:08AM

via free access 
6.0

DIE MORPHOSYNTAKTISCHE STRUKTUR

Aronoffs $(1976,21)$ Behauptung, daB jedem $z u$ bildenden wort ein bereits existierendes zugrunde liege, und daB das bereits existierende sowie das $2 u$ bildende wort den Oberklassenmerkmalen angehörten, rechtfertigt die von Laskowski $(1981,117)$ gestellte Frage, wie denn aufgrund dieser Behauptung z.B. ein Pronomen gebildet werden sollte. Wird dem $z u$ bildenden pronomen ein Nomen zugrunde gelegt, so gehört nur das Ausgangswort mit dem Merkmal $\mathrm{N}$ den Oberklassenmerkmalen an, die auf $\mathrm{N}, \mathrm{V}, \mathrm{A}$ und $\mathrm{P}$ beschränkt sind. Da Aronoff seine Behauptung auf reguläre Wortbildungsprozesse beschränkt, müBte demnach die Bildung der pronomina als irregulärer Proze $\beta$ aus dem Regelapparat ausgeschlossen werden.

Aber Aronoff zählt auch das Adverb - im Gegensatz zu Chomsky (u.a. 1970) und Jackendoff (1977) - zu den Oberklassenmerkmalen. Die Tatsache, daB das ADV als lexikalisches Kategorienmerkmal in der Wortbildung eine Rolle spielt, steht auBer Zweifel selbst dann, und gerade dann, wenn es im Polnischen dazu dient, ein Wort mit dem Merkmal ADV von einem Wort mit dem Merkmal A zu bilden; so z.B. mittels der adverbialen K-Repräsentation $\{+, e+\}$ in pieknie von piqkn-y '(ADV, A) schön'. Wenn also ADV zu den Wortbildungsmerkmalen gezählt werden muB, weshalb sollte dann das Pronomen ausgeschlossen werden? Zumal es im Polnischen eine ganze Reihe von Pronomina gibt. die im Grunde genommen vom pronomen abgleitete Adjektive sind, wie z.B. das sog. Reflexivpronomen swoj' 'sein' oder die sog. Possessivpronomina $m \delta j$, twoj, nasz, wasz 'mein, dein, unser, euer' 44 Diese Behauptung kann dann belegt werden, wenn dem Pronomen im Polnischen generell die struktur (51) zugrunde gelegt wird.

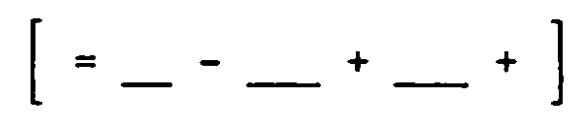

Anhand der aus Klammern und Morphengrenzen bestehenden struktur (51) läßt sich eine Basis aufstellen, die für die Bildung säntlicher Pronomina im Polnischen gilt, d.h. eine Basis, die der Bildung sämtlicher Pronomina im Polnischen zugrunde gelegt werden kann. Wenn $\underline{C}$ für Konsonant und $\underline{V}$ für Vokal stehen, die 
Klammern ( ) für fakultativ und die Grenzen - entsprechend (13) - [- _+] für Wurzel, [+ _+ für Suffix, dann gilt für die Pronomina im Polnischen die Basis (52).

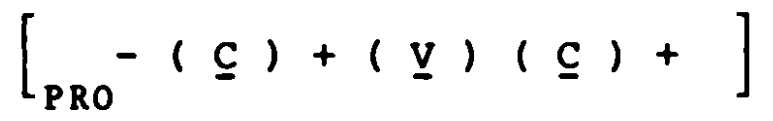

In (52) entsprechen also $\{-(\underline{C})+\}$ der wurzel und $\{+(\underline{V})$ $(\mathrm{C})+\}$ dem pronominalen Suffix. Die Fakultativklammern weisen darauf hin, daB der Konsonant oder der Vokal auch durch Null ersetzt werden können.

wird z.B. die wurzel in (52) durch $\{-s+\}$ ersetzt und das pronominale Suffix - dem inhärenten Merkmal [+ possESSIV] entsprechend - durch $\{+v+\}$, so kann durch Hinzufügung des adjektivischen Suffixes $\{+\mathrm{u} / 0 j+\}$ das possessivische $A$ swoj 'sein' gebildet werden. Wurzel und pronominales Suffix bilden also den Wortstamm, dem das adjektivische suffix hinzugefügt wird. Wird aufgrund des Merkmals [- REFLEXIV] die Wurzel durch $\{-t+\}$ ersetzt, so laBt sich twoj 'dein' bilden. Das Merkmal [- possessiv] hat zur Folge, daB die wurzel durch $\{-m+\}$ und das pronominale suffix durch $\{+\emptyset+\}$ ersetzt werden kơnnen, um moj 'mein' zu bilden."

Die Struktur (51) liegt jedoch nicht nur (52) zugrunde, sondern jedem $z u$ bildenden Wort der polnischen Sprache. Die Struktur (51) setzt sich aus segmenten zusammen, die durch ihre stellung eine bestimmte Funktion in der Wortbildung erfullen. Ich nenne diese struktur die morphosyntaktische struktur; und es gilt folgende Behauptung:

(53) Wenn W-Marker (15) einen lexikalischen Formationsteil erzeugt und wenn sich dieser aus den segmenten = $L^{+}{ }_{-}^{+}{ }_{+}^{+} \ldots$ zusammensetzt, wobel das letzte Segment dem Klassifikator $K$ entspricht, dessen hintere Grenze zugleich die vordere Grenze des grammatischen Formationsteils + _ ist, dann liegt jedem existierenden und jedem $z u$ erzeugenden Wort $w$ die morphosyntaktische Struktur $z$ zugrunde.

Die morphosyntaktische struktur setzt sich also zusammen aus einer Wurzel, einem Klassifikator nebst anderen zu klassifizie- 
renden Segmenten, sowie einem grammatischen Formationsteil. So gilt statt (51):

$$
\mathrm{z} \rightarrow[=\ldots-\ldots+\ldots+\ldots+\ldots+\ldots] / \mathrm{X}-\mathrm{Y}
$$

Der lexikalische Formationste1l des $\mathrm{W}$-Markers (15) bestimmt in (54) den wortbildungsteil, der grammatische Formationsteil hingegen den wortbeugungsteil der morphosyntaktischen struktur $\mathrm{Z}$. Dies gilt unabhängig davon, ob es sich um die Bildung einer lexikalischen Hauptkategorie handelt oder z.B. um die Bildung des pronomen. 46 Der Wortbildungsteil ist Voraussetzung für die Basis-Struktur des Worts. Der Wortbildungsteil wird durch Regeln bestimmt, die die Basis-struktur des Worts erzeugen; diese Regeln sind stets an der morphosyntaktischen Struktur $z$ in (54) orientiert. Die Basis-struktur des Worts wird in folgenden $w$ -Basis genannt.

Die lexikalische Kategorie kennzeichnet die Klammern sowohl des existierenden als auch des $z u$ bildenden worts und bestimmt - neben anderen Merkmalen - die zuordnung der jeweiligen K-Repräsentation. Die lexikalische Kategorie kennzeichnet also den wortbildungsteil der W-Basis.

Der Wortbildungsteil des perfektiven Verbs wypracowac 'ausarbeiten' z.B. hat folgende W-Basis:

$$
\left[\begin{array}{l}
\mathrm{v} \\
\mathrm{v} i
\end{array}\right.
$$

Das von (55) gebildete Nomen wypracowanie 'Ausarbeitung, Schulaufsatz' hat folgende W-Basis:

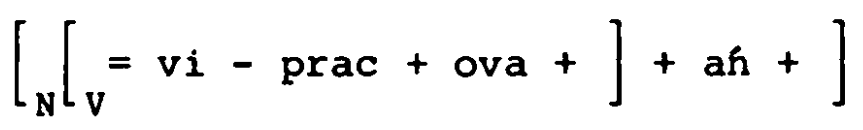

Das Merkmal der lexikalischen Kategorie bestimmt jeweils die morphonemische K-Repräsentation - im folgenden entsprechend § 6.2.1.3 Klassifikator genannt - in den Grenzen $\{+\ldots+\}$; $V$ bestimmt in (55) den V-Klassifikator \{t ova +$\}, N$ bestimmt in (56) den $\mathrm{N}-\mathrm{Klassifikator}\{+a \hat{f}+\}$, während $V$ in (56) den verbalen stamm bestimmt.

Vergleicht man (52) mit (55) und (56), so lassen sich drei unterschiedliche Repräsentationen der W-Basis feststellen; dies 
bezüglich der lexikalischen Kategorienmerkmale. Was die Notation der Morphoneme betrifft, so ist (52) durch morphonemische Alternationsstellen gekennzeichnet, die je nach $\mathrm{W}-\mathrm{Basis}$ des $\mathrm{z}$ bildenden Pronomen durch Morphoneme ersetzt werden können. Diese Alternationsstellen entsprechen phonologischen Merkmalen, die mit der jeweiligen Bedeutung des zu bildenden Pronomen assozilert werden. Durch phonologische Merkmale lassen sich auch (55) und (56) darstellen. Demnach wird die W-Basis durch die morphonemische struktur des existierenden wie des $z u$ bildenden Worts bestimmt. Allen drei genannten W-Basis-Repräsentationen liegt die morphosyntaktische struktur 2 zugrunde.

Die Wortbildung im Polnischen ist also keineswegs an die lexikalischen Hauptkategorien gebunden; sie betrifft alle lexikalischen Kategorien. Anhand der morphosyntaktischen Struktur (54) kann nämlich ebenfalls die w-Basis einer Präposition oder eines deiktischen Ausdrucks gebildet werden, ohne die Notwendigkeit, zwischen einem regulären und einem irregulären wortbildungsprozeB unterscheiden zu müssen. Im Falle der Deixis z.B. ist nicht die Wortbildung, sondern die Wortbeugung irregulär, wenn man z.B. das deiktische pronomen $j a$ ' $1 \mathrm{ch}$ ' berücksichtigt, dessen Flexionsformen mnie und $m n q$ in den Kasus obliqui lauten. In diesen Fällen muB darauf verzichtet werden, das Morphem als selbständige bedeutungstragende Einheit zu sehen. Statt dessen muB von der Assoziation ausgegangen werden, die zwischen bestimmten Einsetzungen - im Sinne von morphonemischen Realisationen der morphosyntaktischen struktur (52) - und der Bedeutung bestehen. will man dennoch im Falle des deiktischen $j a$ vom Flexionsparadigma ausgehen, um den Wortstamm morphemisch $2 u$ bestimmen, so kann die morphonemische Repräsentation des Wortstammes $\{-j+\}$ als Ersetzung des Wortstammes in den Kasus obliqui $\{-m+\}$ oder umgekehrt gesehen werden; siehe dazu Pohl (1984,201ff.).

Obgleich in (55) und (56) zwischen dem existierenden und dem zu bildenden Wort unterschieden wird, bleibt Aronoffs Behauptung, das worter von wortern gebildet werden, dahingestellt. Diese Behauptung hätte dann ihre Gültigkeit, wenn die Wortbildung auf die Derivation eingeschrănkt würde, derzufolge das Lexikon úber eine Reihe von "Ausgangswörtern" verfügt, von denen andere wörter (= Derivate) abgeleitet werden. Die Wortbildung brauchte 
sich dann nur auf die zu derivierenden worter zu beschränken, während der Mechanismus der Bildung von "Ausgangswörtern" als gegeben vorausgesetzt wỉrde. So ist anzunehmen, daB im Polnischen z.B. das Adjektiv konski 'Pferd-' von kon 'Pferd' abgeleitet ist. Doch wie wird kon gebildet, von welchem Wort? Nach Jackendoffs (1975) Full-entry-Theory - auf die sich auch Aronoff stützt - entspräche kon der phonemfolge/koh/ mit dem lexikalischen Merkmal $\mathrm{N}$ sowie den entsprechenden syntaktischen und semantischen Merkmalen. Auf diese Weise wird Halles (1973) Ausnahme-Filter (vgl. (37)) überflüssig; denn kon wird dem Lexikon als vollspezifizierte Einheit entnommen, wăhrend die Redundanzregeln, getrennt, nicht die Erzeugung, sondern die Evaluation bestimmen.

Bei aller Willkürlichkeit, die den ProzeB des Bezeichnens innerhalb einer Sprache ausmacht, mussen die Relationen der morphosyntaktischen und der morphonemischen struktur Gegenstand der Beschreibung der Wortbildung sein; dies selbst auf die Gefahr hin, daB Bedenken laut werden, ob der Sprachkompetente diese strukturen wortbildend realisiert oder nicht. Die Wortbildungsregeln zeigen die Bildung jedes Worts, das im Lexikon als aktuelles oder nicht mehr gebräuchliches oder mögliches enthalten ist.

Bezogen auf das Polnische liegt der Bildung eines jeden Worts die morphosyntaktische struktur 2 in (54) zugrunde; diese setzt sich aus Segmenten zusammen, die bezüglich stellung und Funktion im Hinblick auf die jeweils zu erzeugende $W$-Basis $2 u$ klassifizieren sind; diese Segmente werden im folgenden Morphem- Marker genannt.

Zusammenfassend läbt sich feststellen, daB die morphologische Komponente - vgl. $\S 3.0$ - aus dem W-Marker (15) besteht, der die morphosyntaktische struktur (54) erzeugt; die morphosyntaktische struktur setzt sich aus Morphem-Markern zusammen, die als Voraussetzung für die Bildung der morphonemisch repräsentierten $W$-Basis dienen. Die $W$-Basis entspricht also der morphonemischen Repräsentation des zu bildenden Worts. Der W-Marker erzeugt den Wortbildungsteil und den Wortbeugungsteil der morphosyntaktischen struktur 2 . 


\section{1 DER WORTBEUGUNGSTEIL}

Voraussetzung für den Wortbeugungsteil ist der Wortbildungsteil; denn - so jedenfalls in den meisten slavischen Sprachen und so auch im Polnischen - uber die jeweilige morphonemische Repräsentation des Flektivs entscheiden die inhärenten Merkmale des Wortbildungsteils. So entscheiden z.B. bei den Maskulina die Merkmale [+ BELEBT; + MENSCHLICH] im NOM PL über den Konsonantenwechsel $\{$,$\} - vgl. \$ 2.3$ - in pracownicy 'Arbeiter', NOM SG pracownik, oder in Murzyni $i$ 'Neger', NOM SG Murzyng, oderaber in zydzi 'Juden', NOM SG $z y \underline{d}$; dies im Gegensatz zu [+ BELEBT; - MENSCHLICH] in szczury 'Ratten', NOM SG szczur. "7

Der Wortbeugungsteil ist Gegenstand der Flexion, die aus Kasusregeln ${ }^{48}$ und aus Flexionsregeln besteht. Die Kasusregeln bestimmen die Merkmale, die in den jeweiligen knoten der Satz-Basis, bei Tiefenkasus, und der seichtstruktur, bei strukturalen Kasus, eingesetzt werden, um den entsprechenden Kasus zuzuordnen. Die Flexionsregeln bestimmen anhand der eingesetzten Kasusmerkmale und anhand der inhärenten Merkmale des Wortbildungsteils die morphonemische Repräsentation des Flektivs.

Der Wortbeugungsteil wird hier als gegeben vorausgesetzt. Für die morphonemische Repräsentation des nominalen und des adjektivischen Flektivs im Polnischen gelten folgende Repräsentationsmöglichkeiten:

(57) (a) Vokal: $\{+\underline{V} \#\}$

(b) Vokal und Konsonant: $\{+\underline{v} \underline{C} \#\}$

(c) Vokal, Konsonant und Vokal: $\{+\underline{v} \underline{\mathrm{V}} \#\}$

(d) morphonemisch nicht repräsentiert: $\{+\emptyset \#\}$

Gegenstand der Nominalisierung im Polnischen ist der - gegenüber den Kasus obliqui - unmarkierte NOM SG, beim Pluraletantum der NOM PL. ${ }^{49}$ Der NOM SG gilt auch für das Adjektiv. Für das verb gilt der unmarkierte Infinitivus.

\subsection{DER WORTBILDUNGSTEIL}

Der Wortbildungsteil der morphosyntaktischen struktur 2 - vgl. (54) - ist Gegenstand der Wortbildung. Der Wortbildungsteil enthält die Grundvoraussetzungen für die aufzustellenden wortbildungsregeln. Um diesen Grundvoraussetzungen gerecht werden 
zu können, müssen zunächst die Morphem-Marker, die als Segmente des wortbildungsteils fungieren, klassifiziert werden.

\subsubsection{DIE MORPHEM-MARKER}

Die Morphem-Marker sind durch indizierte Klammern und durch die in (13) definierten Grenzen gekennzeichnet. Die Morphem-Marker zeigen an, welches Morphem an welcher Stelle der W-Basis einzusetzen ist. Die Klassifizierung der Morphem-Marker geschieht innerhalb der Grenzen der morphosyntaktischen Struktur $z$ in (54).

Es wird zwischen folgenden Morphem-Markern unterschieden:

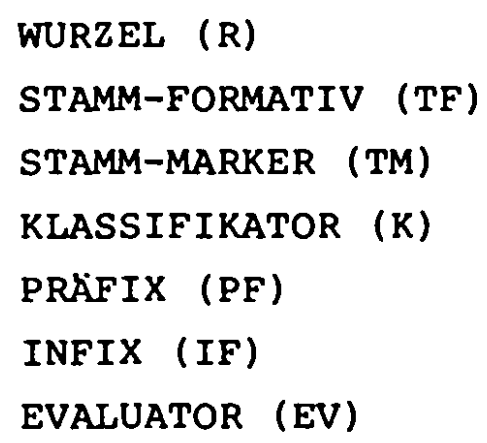

\subsubsection{DIE WURZEL ${ }^{\circ}(\mathrm{R})$}

Der Morphem-Marker $R$ bestimmt die Wurzel der $W$-Basis. Innerhalb des Lexikons repräsentiert $R$ das segment, das einer wortfamilie gemeinsam ist; z.B. in mur - murowas - zamurowas - murarz - murarski 'Mauer, mauern, zumauern, Maurer, Maurer-'. R wird durch seine Grenzen $\left[R^{-}++\right]$definiert.

Wird 2.B. die Morphonemfolge $\{-$ mur +$\}$ mit einer 'Mauer' innerhalb der auBersprachlichen Wirklichkeit assoziiert, so geschieht dies erst nachdem der nominale Klassifikator $\{+\emptyset+\}_{N}$ hinzugefügt worden ist. Das gleiche gilt z.B. für das Adjektiv koci 'Katzen-'; hier wird der Morphonemfolge $\{-\operatorname{kot}+\}$ zunächst der nominale Klassifikator $\{+\emptyset+\}_{N}$ und anschließend der adjektivische Klassifikator $\{+,+\}$ hinzugefügt, der nebst Auslautwechsel die lexikalische Kategorie A bestimnt.

$R$ gilt gegenüber den anderen Morphem-Markern als unmarkiert, da er nichts uber die lexikalische Kategorie aussagt. Die Behauptung, daB $R$ unmarkiert ist, stützt sich auf die allgemein geltende Hypothese, daB die Syntax voraussetzung fur die semantik ist. ${ }^{50}$ 


\subsubsection{DAS STAMM-FORMATIV (TF)}

Der Morphem-Marker TF ist ausschlieblich phonologisch motiviert, d.h., daB er in der Wortbildung allein morphemergänzende Funktion hat. TF wird dann dem Wortstamm zugefugt, wenn dieser vokalisch auslautet. Als Beispiele können hier die Wörter sypialnia 'Schlafzimer' und myjnia 'Autowaschstelle' genannt werden. In sypialnia wird dem vokalischen Verbalstamm ein $\left\{+1^{\prime}+\right\}$, in myjnia wird dem vokalischen Verbalstamm ein $\{+j+\}$ hinzugefügt. Diese Hinzufügungen stellen phonologisch motivierte "Bindeglieder" dar, die ganz offensichtlich damit zusammenhängen, daB der Stammauslaut im Polnischen - und auch in anderen slavischen Sprachen ${ }^{51}$ - bei Hinzufügung eines suffixes nichtvokalisch sein muB.

TF wird durch die Grenzen $\left[_{\mathrm{TF}}{ }^{+}+{ }_{-}\right]$gekennzeichnet.

\subsubsection{DER KLASSIFIKATOR (K) UND DER STAMM-MARKER (TM)}

Der Morphem-Marker $\mathrm{K}$ bestimnt die zuordnung der $\mathrm{W}$-Basis $\mathrm{zu}$ der jeweiligen lexikalischen Kategorie. Seine hintere Grenze entspricht der vorderen Grenze des Wortbeugungsteils - vgl. $\$ 6.1$; diese stellung gilt dann, wenn kein Diminutiv oder Augmentativ vorliegen - vgl. $\$ 6.2 .1 .6$.

K wird durch die Grenzen $\left[\mathrm{K}^{+}{ }_{-}^{+}\right]$und die lexikalische Kategorie des $z u$ bildenden Worts gekennzeichnet.

$\mathrm{K}$ wird realisiert durch:

(59) (a) eine bestimnte morphonemische Repräsentation;

(b) eine morphonemische Nicht-Repräsentation, d.h. durch das sog. Null-Morphem.

Beispiele:

(60) (i) $\left[_{N}=-\operatorname{malp}+\varnothing+\right] \quad$ mazp-a 'Affe'
(ii) $\left[_{N}\left[_{N}=-\operatorname{malp}+\emptyset+\right]+\right.$, ar +$]$ mazpiarz 'Nachäffer'
(1ii) $\left[_{A}\left[{ }_{N}=-\operatorname{malp}+\varnothing+\right]+*+\right]$ mazp-i 'Affen-'

Allen drei $w$-Basen liegt der nominale stam von mazpa zugrunde, der durch die $\mathrm{K}$-Repräsentation $\{+\emptyset+\} \mathrm{N}$ als nominaler stamm klassifiziert ist. In $(60(i i))$ ist es die $k$-Repräsentation $\{+, a \dot{r}+\}_{N}$ die den nominalen stamm nominalisiert, in (60(1ii)) 
ist es die $\mathrm{K}$-Repräsentation $\{+*,+\}_{A}$, die dasselbe Nomen adjektiviert. Das segment [ = -] ist in (60) morphonemisch nicht repräsentiert; es entspricht dem Morphem-Marker PF - vgl. $\S 6.2 .1 .4$.

Uber die Klassifizierung eines worts durch eine lexikalische Kategorie entscheidet allein $\mathrm{K}$. Alle anderen, im Wortstam repräsentierten Klassifikatoren, die vor $K$ stehen - in $(60(i i))$ und (60(iii)) ist es jeweils das sog. Null-Morphem - haben die Funktion von Stamm-Markern TM. So zeigt TM in $(60(i 1))$ an, daB das Nomen von einem Nomen gebildet ist, in $(60(i i i))$ zeigt TM an, daB das Adjektiv von einem Nomen gebildet ist.

Während $K$ darüber entscheidet, in welchen terminalen Knoten das Wort einzusetzen ist, entscheidet TM daruber, welche Wortbildungsregel anzuwenden und welche $\mathrm{K}$-Repräsentation hinzuzufügen ist, dies zusammen mit den inhärenten Merkmalen.

\subsubsection{DAS PRAFFIX (PF)}

Der Morphem-Marker PF wird durch die Grenzen $\zeta_{\mathrm{PF}}=\ldots-$-] gekennzeichnet. Wird PF durch zwei Morpheme repräsentiert, so gelten die Grenzen $\left[_{\mathrm{PF}}=\ldots-\ldots-\right.$ ], so z.B. in po-prze-rywać '(an einigen Stellen) unterbrechen'. Die morphonemische Repräsentation des PF hat - nach Isačenko (1975,359) - eine einengende qualifizierung der Bedeutung zur Folge. Es wäre daher zu uberlegen, PF durch Modifikator zu ersetzen; diese Ersetzung wirde jedoch erst bei der Diskussion des Verbs aktuell, da PF eine wichtige Rolle beim sog. Aspekt sowie bei den Aktionsarten im Polnischen spielt.

\subsubsection{DAS INFIX (IF)}

Der Morphem-Marker IF wird durch die Grenzen [ $\mathrm{IF}^{+}{ }_{-}^{+}$] gekennzeichnet. Seine stellung bezieht er entweder unmittelbar vor der verbalen $\mathrm{K}$-Repräsentation, z.B. in odskak-iw-ad' (etwa: wiederholt) abspringen', wypi-j-ac '(wiederholt) austrinken', oder innerhalb der $\mathrm{K}$-Repräsentation, z.B. in opracow-yw-ac' '(wiederholt) bearbeiten'. Laut Isačenko $(1975,359)$ liegt in diesen Verben eine sekundäre Imperfektivierung vor; diese ist - in der Regel - aufverben beschränkt, die bereits ein imperfektives Gegen- 
stück zu dem - in der Regel - präfigierten perfektiven Aspekt haben: vgl. z.B. pracowac - wypracowac '(ipf.) arbeiten - (pf.) ausarbeiten'. IF hat also eine iterativierende Funktion, indem durch seine Einsetzung die Möglichkeit gegeben ist, die vom Verb bezeichnete Tätigkeit quantitativ zu bestimmen.

\subsubsection{DER EVALUATOR (EV)}

Der Morphem-Marker EV ist ausschlieBlich semantisch motiviert. EV wird dann dem Wortbildungsteil zugefügt, wenn die K-Repräsentationen von $\mathbf{N}$ oder $A$ realisiert werden und ein Diminutiv oder ein Augmentativ gebildet werden soll. EV bezieht seine Stellung hinter dem $k, d . h$. seine vordere Grenze entspricht der hinteren Grenze des $K$, während seine hintere Grenze der vorderen Grenze des Wortbeugungsteils entspricht. EV verändert weder die lexikalische Kategorie noch die inhärenten Merkmale. Die Annahme des EV ist insofern sinnvoll, als es in der polnischen Gegenwartssprache - besonders in Krakau - eine unüberhör- und -sehbare Neigung zum Diminutiv gibt: masezko 'Butter', jajeczko 'Ei', szyneczka' 'schinken' u.v.a.m. ${ }^{\text {s2 }}$

\subsection{DIE MORPHOSYNTAKTISCHE STRUKTUR (61)}

Anhand der in $\S 6.1$ - $\S 6.2 .1 .6$ klassifizierten Morphem-Marker kann eine morphemmarkierte morphosyntaktische struktur $z$ angenommen werden, die - entsprechend (54) - der W-Basis jedes zu bildenden worts im Polnischen zugrunde liegt.

Wenn WT für Wortbildungsteil, FT für Wortbeugungsteil und $\underline{X}$ _ $\underline{Y}$ furr Umgebung stehen, dann gilt (61):

(61)

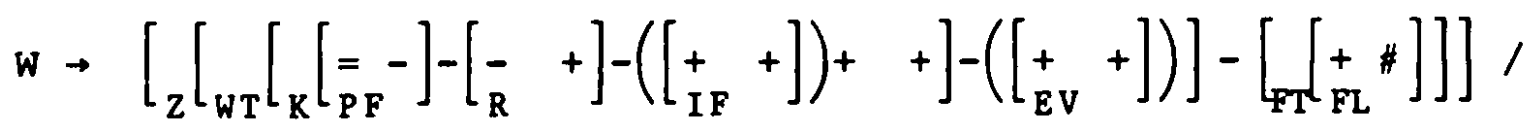

$$
\begin{aligned}
& \text { / } \underline{X}
\end{aligned}
$$

Der Nominalisierung im Polnischen wird folgende Feststellung zugrunde gelegt; vgl. $\$ 6.0$ :

(62) Das Wort wird aus Morphemen gebildet, denen die als Morphem-Marker klassifizierten Segmente innerhalb WT der morphemmarkierten struktur (61) zugrunde liegen. 


\subsection{WORTBILDUNG}

Die Wortbildung beschreibt die Bildung von existierenden - darunter auch die sog. alten und veralteten, d.i. in der Gegenwartssprache nicht mehr aktuellen - und von möglichen wörtern einer Sprache. All diese wörter sind jedoch Einheiten des Lexikons dieser sprache. Die Wortbildung wird in Regeln dargestellt, die hier zunächst WB-Regeln genannt werden. Für die Wortbildung gilt allgemein, daß sie eine begrenzte Anzahl von WB-Regeln für eine unbegrenzte Anzahl von Wörtern bereit hält. Diese Behauptung schließt aus, daß für jedes einzelne wort im Lexikon eine gesonderte Regel aufgestellt werden muß. Die wörter bilden bezüglich der aufzustellenden bzw. anzuwendenden WB-Regeln Gruppen, im Sinne von geordneten Mengen. Grundsätzlich ist festzustellen:

Die WB-Regeln geben die morphonemische Repräsentation der jeweiligen Morphem-Marker für die Bildung der W-Basis an.

Dies geschieht aufgrund der Behauptung (62), wobei die W-Basis der morphonemischen struktur des zu bildenden worts entspricht.

Die Ersetzung des jeweiligen Morphem-Markers durch die jeweilige morphonemische Repräsentation geschieht mittels Merkmalen. Diese Merkmale erfordern die Einbeziehung der Wortphonologie - vgl. $\S 2$-, die in einem gesonderten, an der Wortbildung orientierten Regelsystem in $\S 7.1 \mathrm{ff}$. dargestellt wird, der Morphologie - vgl. $\S 3$ und $\S 6$ - sowie der Wortsemantik - vgl. $\S 4$-, die in $\S 7.2 .4$ in die inhärenten Merkmale einbezogen wird.

\subsection{MORPHONOLOGIE DES POLNISCHEN}

Die Morphonologie des Polnischen wird hier im Hinblick auf die Wortbildung auf das wesentliche zusammengefaßt. ${ }^{53}$ Die Morphoneme des Polnischen werden zunächst mit Hilfe binärer phonologischer Merkmale klassifiziert. Diese Merkmale sind an der Artikulation des Polnischen orientiert und dienen als Bedingungen für die phonetisch und für die morphologisch motivierten Alternationen, die als Regeln dargestellt werden.

Die Morphonologie des Polnischen setzt sich also zusammen aus: 
(64) (a) der Bestimmung eines merkmalphonologischen Inventars der Morphoneme, die in der $W-B a s i s$ als Segmente fungieren und Bündeln binärer, phonetisch motivierter Merkmale entsprechen;

(b) phonetisch motivierten Alternationsregeln;

(c) morphologisch motivierten Alternationsregeln.

\subsubsection{MERKMALPHONOLOGISCHES SYSTEM DES POLNISCHEN}

In der generativistischen Unterscheidung zwischen dem System Sprache und deren Aktualisierung, also in der Unterscheidung zwischen Kompetenz und Performanz, wird der Idealfall eines kompetenten Sprecher-Hörers angenommen. ${ }^{54}$ Dieser Idealfall wird hier dem der Polszczyzna kulturalna - wie sie u.a. von Benni (1959) aufgefaßt wird - Kundigen gleichgesetzt; eine Unterscheidung $z$ wischen bestimmten Standards des Polnischen und deren $\mathrm{Zu}-$ ordnung $\mathrm{zu}$ bestimmten sozialen schichten steht hier also nicht zur Debatte. Gegenstand ist hier lediglich die Kultursprache, die den jeweiligen varianten als Korrektiv dient. 55

In Anlehnung an Chomsky-Halle (1968), Paulsson (1974) und Laskowski (1975) werden für das Morphoneminventar des Polnischen folgende 15 phonetisch motivierte Merkmale unterschieden:

(65) Oberklassenmerkmale: [ \pm OBSTRUENT]

[ \pm SILBISCH]

[ \pm KONSONANTISCH ]

$\begin{array}{ll}\text { Resonanzmerkmale: } & {[ \pm \text { ANTERIOR }]} \\ \text { (Artikulationsstel- } & {[ \pm \text { KORONAL }]} \\ \text { le/ Artikulations- } & {[ \pm \text { LATERAL }]} \\ \text { organ) } & {[ \pm \text { PALATAL }]} \\ & {[ \pm \text { NASAL }]} \\ & {[ \pm \text { HOCH }]} \\ & {[ \pm \text { NIEDRIG }]} \\ & {[ \pm \text { VORN }]}\end{array}$

Artikulationsart: [ \pm DAUERND]

[ \pm FRIKATIV]

[ \pm VIBRATOR]

Artikulationsquelle: [ \pm STIMMHAFT] 


\section{1 .1 .1 DIE OBERKLASSENMERKMALE}

Bei den Oberklassenmerkmalen in (65) wird zwischen Obstruenten und Sonoranten unterschieden, d.h. [- OBSTRUENT] entspricht [+ SONORANT]. Diese Unterscheidung ist im Polnischen u.a. dadurch gerechtfertigt, daß zu den Sonoranten nicht nur Vokale, sondern auch solche Konsonanten gehören, die im Gegensatz zu den ebenfalls konsonantischen obstruenten keine Geräuschlaute sind. Das Merkmal [+ KONSONANTISCH] impliziert im Polnischen [- SILBISCH] u.a., weil es im Polnischen keine silbenbildenden Konsonanten gibt; im Polnischen wird der silbennukleus ausschließlich durch einen Vokal repräsentiert, daher bleibt das Merkmal [+ SILBISCH] den Vokalen vorbehalten. Demzufolge lassen sich auch die Nasalkonsonanten und die Liquida bezüglich der Oberklassenmerkmale nicht differenzieren; beide bleiben als Konsonanten positiv, als silbenbildende segmente und Geräuschlaute negativ markiert; ihre Differenzierung wird u.a. durch das Artikulationsmerkmal der Nasalität bestimmt. Zur Veranschaulichung (66):

\begin{tabular}{|l|c|c|c|c|}
\cline { 2 - 4 } & \multicolumn{2}{|c|}{ S O N O R A N T E N } & $\begin{array}{c}\text { OBSTRU- } \\
\text { ENTEN }\end{array}$ \\
\cline { 2 - 5 } & GLEITL. & $\begin{array}{l}\text { NASALKONS . } \\
\text { /LIQUIDA }\end{array}$ & VOKALE & + \\
\hline OBSTRUENT & - & - & - & - \\
\hline SILBISCH & - & - & + & + \\
\hline KONSONANTISCH & - & + & - & + \\
\hline
\end{tabular}

Der weder obstruentische noch silbische noch konsonantische Gleitlaut in (66) entspricht dem Morphonem $\{j\}$, das dem weder vokalischen noch konsonantischen Laut [i] entspricht. Seine Stellung in der silbe läßt sowohl eine klassifikation als Halbvokal, nämlich als zweites Glied eines Diphthongs zu, z.B. in [olícec] 'Vater', als auch als Konsonant, nämlich als silbenkoda, z.B. in [ $\check{c} \pm i$ ] 'wessen'. Aufgrund dieser "Zwitterstellung" wird dieser Laut bezüglich aller drei Oberklassenmerkmale negativ markiert, und da er nicht silbenbildend ist, wird er wie alle Konsonanten durch $\subseteq$ symbolisiert. 


\subsubsection{DIE LIQUIDA}

Die Liquida $r$ und $z$ können im Polnischen jeweils zwei fakultative Varianten haben: $r$ kann entweder als sog. "Zungen- $R$ " - und dies ist die Norm - oder aber als uvulares [R] artikuliert werden; $z$ als bilabiales [u] - und dies ist die Norm - aber auch - vereinzelt auf der Bühne sowie bei Sprechern der ehemals polnischen Ostgebiete - als apikal-dentales, nichtpalatales $\{1\} .{ }^{56}$ Bezulglich der Artikulationsstelle - vgl. (65) - kann für die Artikulation des Lauts, der $z$ zugrunde liegt und dem das Morphonem $\{1\}$ entspricht, folgendes angenommen werden:

(67.1) (a) WENN [+ ANTERIOR], DANN [ - KORONAL]

(b) WENN [ - ANTERIOR], DANN [ + KORONAL]

Fưr beide Möglichkeiten in (67.1) gilt [- PALATAL]; dies im Gegensatz zu $\left\{1^{\prime}\right\}$.

Für die Artikulation des Lauts, der $r$ zugrunde liegt, gelten die Möglichkeiten:

(67.2) (a) WENN [+ VORN], DANN [+ ANTERIOR, + KORONAL]

(b) WENN [ - VORN], DANN [- ANTERIOR, - KORONAL]

Fưr beide Möglichkeiten in (67.2) gilt [+ VIBRATORj.

Da es sich - wie bereits hervorgehoben - um jeweils zwei fakultative varianten handelt und da jeweils die variante (a) der Norm entspricht, gilt jeweils Variante (a).

Zu den Liquida zählt auch die morphonemische Entsprechung des Graphems $\mathrm{rz}$. Die lautliche Repräsentation, die diesem Graphem zugrunde liegt, entspricht dem alveolaren [i்] nebst seinem stimmlosen Alternanten [亡̈]; so werden die Wörter wie z.B. brzeg 'Ufer', krzak 'Strauch', mierzyc 'messen', zwierz 'Tier' in der

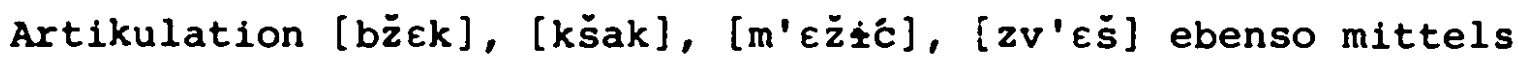

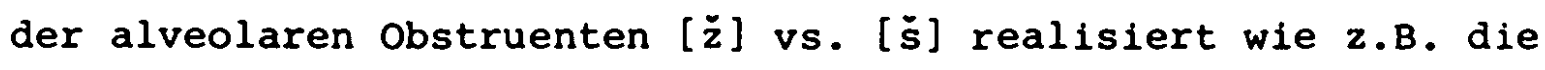
Worter $\dot{z} q d a c$ 'fordern', wainy 'wichtig, gültig', wzyć siq 'sich einleben', pomó '(IMPERATIV SG) helfen', nämlich [żondać],

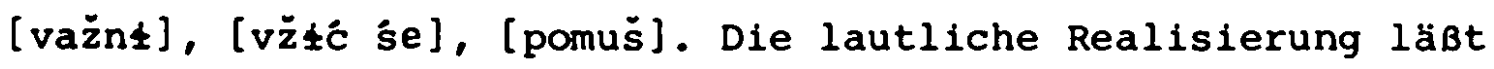
also keine Differenzierung zwischen den Phonemen bzw. Morphonemen, die den Graphemen $r z$ und $\dot{z}$ zugrunde liegen, zu.

Werden die morphologisch motivierten Alternationen berücksich- 


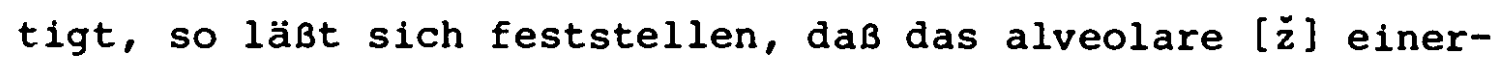
seits mit dem velaren [g], anderseits aber mit dem Liquid [r] alterniert. In beiden Fällen handelt es sich um Alternationen, die sich gegenseitig ausschließen, d.h., daB die Alternation [ż] vs. [g] in anderen Umgebungen durchgeführt wird als die Alternation [ż] vs. [r]. Um diese sich gegenseitig ausschließenden Umgebungen auf der lautlichen Ebene klassifizieren $z u$ wollen, bedürfte es einer stattlichen Anzahl von Regeln, da hier nicht nur die stellung bezüglich der Grenzen bzw. eines anderen Lauts berücksichtigt werden müBte, sondern die stellung bezüglich ganzer Lautketten. Im Hinblick auf die Wortbildung werden daher diese beiden Alternationen durch die Morphoneme $\{\dot{z}\}$ vs. $\{g\}$ einerseits, anderseits durch die Morphoneme $\{r\}$ vs. $\{\check{r}\}$ notiert, wobei eine entsprechende phonetische zusatzregel die Artikulation [ $\check{z}$ ] dem Morphonem $\{\check{r}\}$ zuordnen - siehe dazu auch Pohl (1980a,358ff.) - kann.

\subsubsection{DAS MORPHONEM-INVENTAR DES POLNISCHEN}

In (68) wird das Morphonem-Inventar des Polnischen anhand der Merkmale (65) dargestellt. Die Sonoranten sind darin - wie bereits in (66) - in: Gleitlaut, Nasalkonsonant, Liquid und Vokal unterteilt. Die Obstruenten werden bezüglich ihrer Artikulationsstelle in: Labiale, Dentale bzw. Palatale, Alveolare sowie Velare unterteilt.

Der Labialität der obstruenten entsprechen die Merkmale [+ ANTERIOR, - KORONAL], der Dentalität entsprechen die Merkmale [+ ANTERIOR, + KORONAL], während bei den Palatalen unter den Dentalen noch das Merkmal [+ LATERAL] hinzukommt. Der Alveolarität entspricht die Merkmalkombination [- ANTERIOR, + KORONAL], der velarität dagegen [- ANTERIOR, - KORONAL].

Die Palatalität wird grundsätzlich durch drei Merkmale gekennzeichnet, nämlich durch [+ PALATAL, + LATERAL, + HOCH]. Bezüglich aller drei Merkmale sind die palatalen Dentalen sowie der palatale Liquid positiv markiert. Alle nichtdentalen Palatalen sind sowohl bezüglich der Palatalität als auch der hohen zungenlage positiv, bezüglich der Lateralität dagegen negativ markiert, während die velare, außer $\{x\}$, nur bezüglich der hohen zungenlage positiv, bezüglich Palatalität und Lateralität dage- 


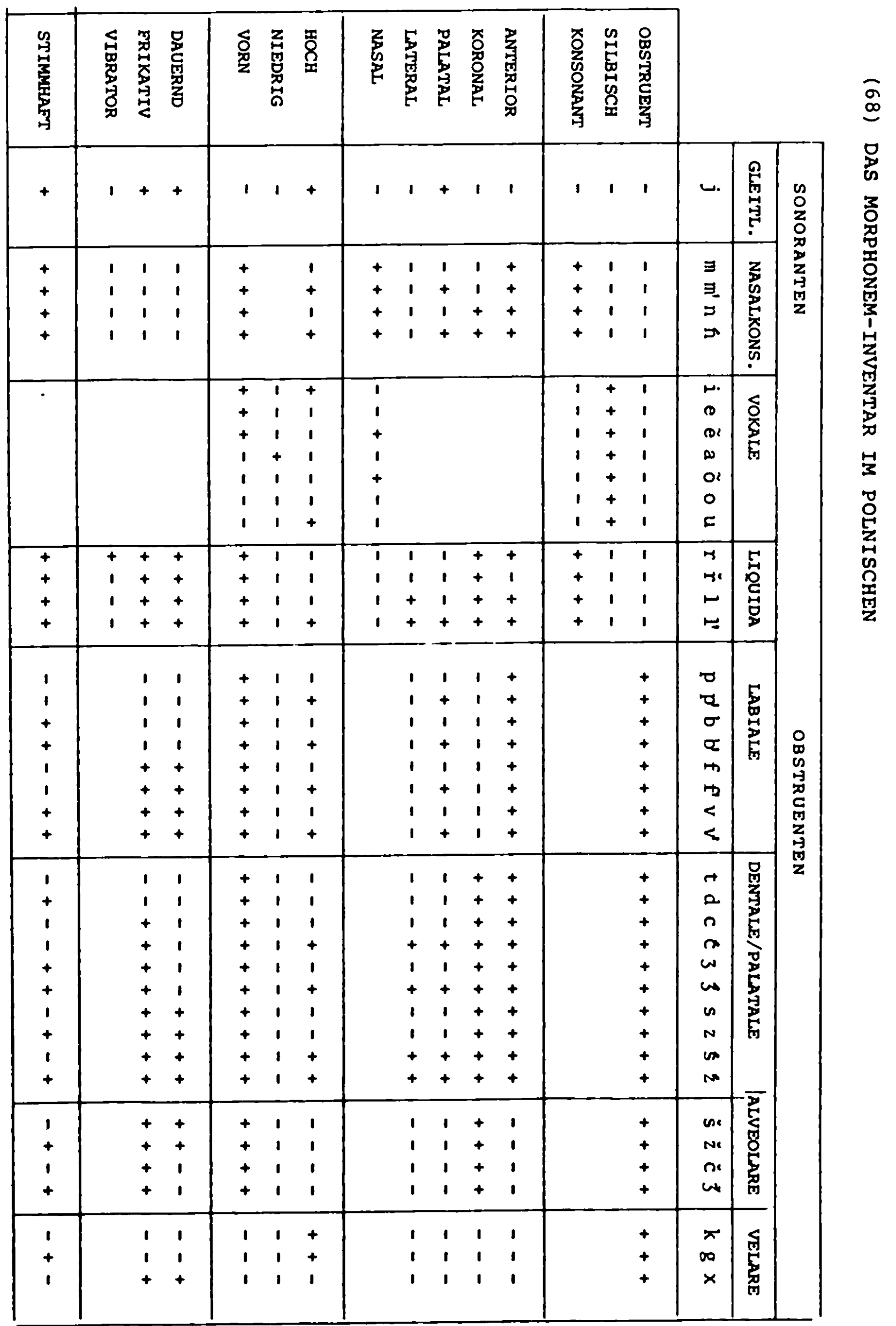


gen negativ. Die hohe Zungenlage bei der Artikulation der Velare [k] und [g] bewirkt nämlich im Polnischen, daB beide Laute in der Regel nur vor [1] und nie vor [ 1 ] stehen; die Lautverbindungen $*[k \pm]$ oder $*[g t]$ sind nicht polnisch.

\subsubsection{DIE MORPHONOLOGISCHEN REGELN}

Die morphonologischen Regeln - vgl. (64) - werden unterteilt in:

(69) (a) PR-Regeln, d.i. phonologische Redundanzregeln;

(b) PT-Regeln, d.i. phonetisch motivierte Alternationsregeln;

(c) PL-Regeln, d.i. morphologisch motivierte Alternationsregeln.

Die PR-Regeln betreffen die zuordnung bestimmter Merkmale innerhalb des Morphonem-Inventars. Dazu gehören z.B. die Kennzeichnung der Divergenz zwischen Obstruenten und Sonoranten. Die PT-Regeln betreffen die Alternationen, die innerhalb der lautlichen Realisierung stattfinden, wie z.B. die progressive Assimilation in [kšak] bei der morphonemischen Repräsentation \{krak\} - krzak 'strauch'. Die PL-Regeln betreffen die durch die Wortbildung (und die Wortbeugung) motivierten Alternationen, wie z.B. in \{'sp'evak\}\} vs. \{śp'evaçk+a\} - spiewak - spiewaczka 'sănger - Sängerin'.

Entsprechend der bereits in (66) zugeordneten Merkmale gilt für die Obstruenten:

$$
\begin{array}{llll}
\text { (PR1.0) } & \text { [+ OBSTRUENT] } & \text { impliziert } & {[- \text { SONORANT ] }} \\
\text { (PR1.1) } & \text { [+ OBSTRUENT] } & \text { schlieBt aus } & {[+ \text { SONORANT ] }} \\
\text { (PR1.2) } & \text { [+ OBSTRUENT] } & \text { impliziert } & {\left[\begin{array}{l}
- \text { SILBISCH } \\
\end{array}\right.}
\end{array}
$$

Unter den Obstruenten ist $\{x\}$ das einzige Morphonem, dem kein stimnaftes Pendant zugewiesen wird; daher gilt:

$$
\text { (PR2) } \quad\left[\begin{array}{l}
- \text { ANTERIOR } \\
- \text { KORONAL } \\
- \text { HOCH }
\end{array}\right] \text { impliziert [- STIMMHAFT] }
$$

Bei den Vokalen kann man davon ausgehen, daß $\{\tilde{e}\}$ und $\{\tilde{\}}\}$ die nasalen Pendants von $\{e\}$ und $\{0\}$ sind. Es sind die einzigen Vokale, deren Nasalitat in bestimnter Ungebung distinktive Funktion 
hat, nämlich vor $[s, z, \dot{s}, \xi, \dot{s}, \dot{z}]$, sonst werden die Nasalvokale entweder leicht nasaliert vor Nasalkonsonanten, ebenso wie andere Vokale in dieser stellung, oder aber völlig entnasaliert, wie [ $\varepsilon$ ] und [0], nämlich vor den Liquida [1] und [1'] artiku-

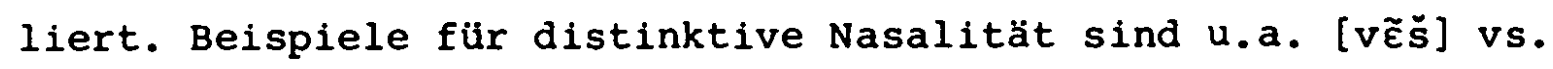

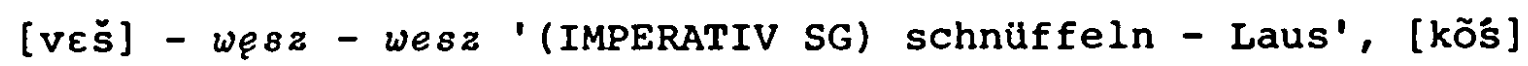
vs. [koś] - kqs - kos '(IMPERATIV SG) beiBen, mähen'. Beispiele für Entnasalierung von Nasalvokalen sind u.a. [l'Ẽnk] - $Z_{e k}$ 'Furcht', [koñt] - kqt 'Ecke, Winkel' sowie [vzol] - wziqz '(3. PERS SG PRÄT MASK) nehmen', [vizla] - wzigza' (3. PERS SG PRÄT FEM) nehmen'.

In den Beispielen $l_{\ell k}$ und $k q q t$ ist die Entnasalierung nicht so stark wie in wzigza und wziqz, wo die Nasalität völlig aufgehoben ist. Die leichte Nasalität in $l_{q k}$ und $k q t$ ist durch den Nasalkonsonanten bedingt, der auch die anderen vokale in dieser stellung entsprechend nasaliert, so z.B. in [ĩndik] - indyk 'Truthahn', [kũnšt] - kunszt 'Kunstfertigkeit', [trãnvaj] - tramwaj 'straßenbahn', [brĩnza] - bryndza 'poln. Schafskäse'. In allen genannten Fällen der Nasalierung nichtnasaler sowie der Entnasalierung nasaler Vokale gelten phonetisch motivierte, d.i. PT - Regeln. Da jedoch auf der morphonemischen Ebene der wortbildung diese Nasalierung keine Funktion hat, wird den hohen und dem niedrigen Vokal das Merkmal [- NASAL] zugeordnet. Dafür gelten folgende PR-Regeln:

$\begin{array}{llll}(\text { PR3 }) & {\left[\begin{array}{l}+ \text { SILBISCH } \\ + \text { HOCH }\end{array}\right]} & \text { impliziert } & \text { [- NASAL] }\end{array}$

Aus den Implikationen (PR3) und (PR4) geht also hervor, daB die beiden hohen Vokal-Morphoneme und das niedrige keine nasalen Pendants haben.

Für die hohe zungelage der velare $\{k\}$ und $\{g\}-v g l$. $\{7.1 .1 .3-$ - gilt folgende PR-Regel:

$(P R 5) \quad\left[\begin{array}{l}- \text { ANTERIOR } \\ - \text { KORONAL } \\ - \text { DAUERND }\end{array}\right]$ impliziert [+ HOCH ] 
Für die Bestimmung des Vibrators - vgl. $\S 7.1 .1 .2$ - beim Liquid \{r \} gilt folgende PR-Regel:

(PR6) $\left[\begin{array}{l}- \text { OBSTRUENT } \\ + \text { ANTERIOR } \\ + \text { KORONAL } \\ - \text { LATERAL } \\ - \text { NASAL }\end{array}\right]$ impliziert [ + VIBRATOR ]

Für die Klassifizierung des Morphonems $\{j\}$ als Sonorant - vgl. $\S 7.1 .1 .1$ - gilt folgende PR-Regel:

(PR7) $\quad\left[\begin{array}{l}- \text { ANTERIOR } \\ - \text { KORONAL } \\ + \text { FRIKATIV }\end{array}\right]$ impliziert $\left[\begin{array}{l}- \text { OBSTRUENT } \\ - \text { SILBISCH } \\ - \text { KONSONANTISCH }\end{array}\right]$

Die Alternation der Velare - vgl. $\S 2.3$ - betrifft die Alternationen k/cz, g/z, ch/sz; so z.B. in: rok / rocznik 'Jahr/Jahrgang', noga / podnózek 'FuB, Bein/FuBbank', ucho / nauszniki 'Ohr/Ohrenschützer'. Die morphonemischen Entsprechungen dieser Alternationen sind: $\{k\}$ vs. $\{\check{c}\},\{g\}$ vs. $\{\check{z}\}$ sowie $\{x\}$ vs. $\{\check{s}\}$, d.h., daß die Alveolare mit den Velaren alternieren. Diese Alternation wird auf der morphonemischen Ebene durch das Symbol $\{*\}$, also durch "sternchen" notiert. Da es sich um eine morphologisch motivierte Alternation handelt, gilt folgende PL-Regel: (PL8)

$\begin{array}{lllll}\{k\} & + & \{*\} & \rightarrow & \{k *\} \rightarrow \\ \{g\} & + & \{*\} \rightarrow & \rightarrow \\ \{x\} & + & \{*\} \rightarrow & \rightarrow & \left\{x^{*}\right\} \rightarrow\end{array}$

Regel (PL8) muß im Hinblick auf die Alternation $c / c z, z . B$. in owca / owczarz 'Schaf/Schäfer' durch (PL9) ergänzt werden:
(PL9)
$\{c\}$
$\{*\}$
$\{c *\} \rightarrow$
$\{\check{c}\}$

Für die ebenfalls morphologisch motivierte Alternation der Liquida $r / r z$ - vgl. $\S 7.1 .1 .2$ - z.B. in para / parzysty 'Paar/paarig' wird das symbol $\{$,$\} , also "Komma" angenommen, und es gilt$ die PL-Regel:

$(\mathrm{PL10})\{\mathrm{r}\}+\{,\} \rightarrow\{r,\} \rightarrow\{\check{r}\}$

Für die Alternation zwischen nichtpalatalem und palatalem Liquid $\{1\}$ vs. $\left\{1^{\prime}\right\}$ wird ebenfalls das symbol $\{$,$\} angenommen:$
(PL11)
(1)

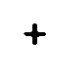
$\{,\} \rightarrow$
$\{1,\} \rightarrow$
$\left\{1^{\prime}\right\}$ 
Abgesehen von einigen neueren Lehnwörtern, wie z.B. plastik 'Plastik (Stoff)', kennt das Polnische weder ein palatales ' $t$ ' ] noch ein palatales 'd']; daher auch die verbreitete Artikulation [pl'ast $4 k]$. Die nichtpalatalen Dentale $\{t\}$ und $\{d\}$ alternieren mit $\{\varepsilon\}$ und $\{\xi\}$, so z.B. skrot / skrocic 'Abkürzung/abkürzen' und in chzod/chzodzic 'Kühle/kühlen'. Für diese Alternationen gilt:

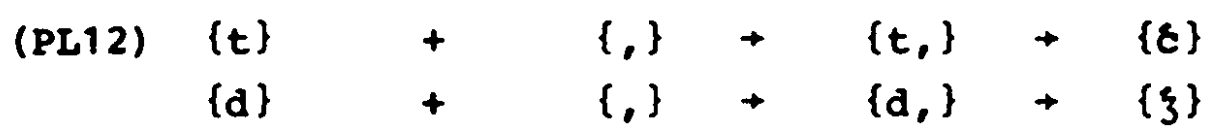

Die velaren Obstruenten alternieren nicht nur - entsprechend (PL8) - mit den Alveolaren; sie alternieren auch - diachronisch vgl. 2. und 3. Palatalisierung - mit Dentalen bzw. Dentalpalatalen. Diese Alternationen lassen sich folgendermaBen veranschaulichen:
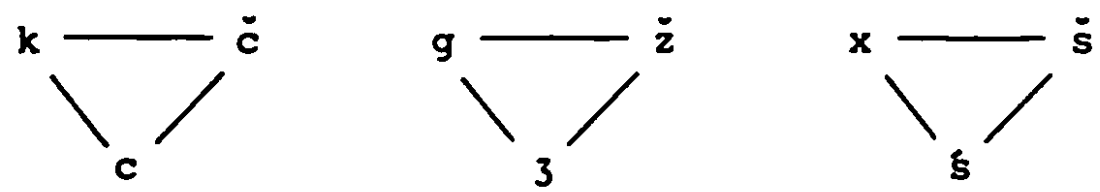

Die waagerecht dargestellte Alternation entspricht - diachronisch vgl. 1. Palatalisierung - der Regel (PL8), wăhrend die Diagonale $c-\dot{c}$ der Regel (PL9) entspricht.

Für die Diagonalen $k$ - $c$ sowie $g-3$ wird ebenfalls das Symbol "Komma" notlert, und es gelten die Regeln:

\begin{tabular}{|c|c|c|c|c|c|c|}
\hline$(P L 13)$ & $\{k\}$ & + & $\{\}$, & $\rightarrow$ & $\{k\}$, & $\rightarrow$ \\
\hline & $\{g\}$ & + & $\{\}$, & $\rightarrow$ & $\{g\}$, & $\rightarrow$ \\
\hline & $\{\check{s}\}$ & + & $\{\}$, & $\rightarrow$ & $\{\check{s}\}$, & $\rightarrow$ \\
\hline
\end{tabular}

Die an'letzter Stelle stehende Alternation entspricht z.B. pastuszy / pastusi 'Hirten-/Hirten'; vgl. (PL8) x- ́s.

Der Vollstăndigkeit halber kann noch für die Diagonale 3 - i, 2.B. In pieniqdz / pieniginy 'Geld/Geld-' folgende Regel angenommen werden:
(PI14)
$\{3\}+$
$\{*\} \quad \rightarrow\{3 *\}$
$\{\check{z}\}$

Für die anderen Alternationen zwischen nichtpalatalen und palatalen Sonoranten und Obstruenten gilt folgende Regel: 


\begin{tabular}{|c|c|c|c|c|c|c|c|}
\hline (PL15) & (v) & + & $\{\}$, & $\rightarrow$ & $\{v\}$, & $\rightarrow$ & $\left\{v^{\prime}\right\}$ \\
\hline & $\{\mathrm{m}\}$ & + & $\{\}$, & $\rightarrow$ & $\{m\}$, & $\rightarrow$ & $\{\mathbf{n}\}$ \\
\hline & $\{n\}$ & + & $\{\}$, & $\rightarrow$ & $\{n\}$, & $\rightarrow$ & $\{$ h $\}$ \\
\hline & $\{p\}$ & + & $\{\}$, & $\rightarrow$ & $\{p\}$, & $\rightarrow$ & $\left\{p^{\prime}\right\}$ \\
\hline & $\{b\}$ & + & $\{\}$, & $\rightarrow$ & $\{b\}$, & + & $\left\{b^{\prime}\right\}$ \\
\hline & $\{f\}$ & + & $\{\}$, & $\rightarrow$ & $\{f\}$, & + & $\left(f^{\prime}\right)$ \\
\hline & $\{c\}$ & + & $\{\}$, & $\rightarrow$ & $\{c\}$, & + & $\{\varepsilon\}$ \\
\hline & $\{3\}$ & + & $\{\}$, & $\rightarrow$ & $\{3\}$, & + & $\{\xi\}$ \\
\hline & $\{\boldsymbol{b}\}$ & + & $\{\}$, & $\rightarrow$ & $\{s\}$, & + & $\{\xi\}$ \\
\hline & $\{x\}$ & 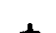 & $\{\}$, & $\rightarrow$ & $\{z\}$, & + & \\
\hline
\end{tabular}

Für den sog. flüchtigen Vokal, der im Polnischen - mit Ausnahme von osioz, kozioz, kocioz 'Esel, ziegenbock, Kessel', die neben den älteren osiez, koziez, kociez stehen - als $\{e\}$ oder als $\{1\}$ realisiert wird, gelten folgende Regeln:

$(P L 16)(1.0)$ Tritt $\{8\}$ sowohl in PF als auch in anderen Morphem-Markern von $\mathrm{z}$ - vgl. (61) - oder in $\mathrm{P}$ und $\mathrm{z} \mathrm{zu}-$ gleich auf, so ist $\{8\}$ nach den Regeln $(1.1)-(1.4)$ $g$ e $n$ a e $1 \mathrm{~nm}$ a $l$ zu realisieren.

\begin{tabular}{|c|c|c|c|c|}
\hline (1.1) & $\{8\}$ & $+\{\varnothing\} /$ & & \\
\hline$(1.2)$ & $\{8\}$ & $\rightarrow\{\underline{v}\} /$ & & \\
\hline$(1.3)$ & $\{8\}$ & $\{\underline{v}\} /$ & & \\
\hline$(1.4)$ & $\{8\}$ & $\{\underline{v}\} /$ & & \\
\hline$(1.5)$ & $\{y\}$ & $\rightarrow\{1\} /$ & & \\
\hline$(1.6)$ & $\{y\}$ & $\{\mathbf{e}\}$ & & \\
\hline
\end{tabular}

Für die anderen Vokalalternationen gelten folgende Symbole:

(70) $\left\{\varepsilon_{1}\right\}$ für die Alternation der Nasalvokale $\{\tilde{e}\}$ vs. $\{\tilde{\}}\}, z . B$. in: pajqk / pajkczyna 'Spinne/Spinnwebe', bzqd / bzq$d y$ 'Fehler (NOM SG/PL)';

$\left\{\varepsilon_{2}\right\}$ für die Alternation $\{a\}$ vs. \{e\}, z.B. In: wiara / / wierzyl / wierze 'Glaube/glauben/Glaube (DAT, LOK SG)';

$\left\{\&_{3}\right\}$ für die Alternation $\{0\}$ vs. $\{u\}, z \cdot B . g z \sigma d / g z o d u /$ / gzodowac 'Hunger (NOM/GEN SG)/ hungern';

$\left\{\varepsilon_{4}\right\}$ für die überlappende Alternation $\{u\}$ vs. $\{a\}$ vs. \{e\} vs. \{0\}, z.B. In chod / chadzac / chodzic 'Gang/gehen (ITERATIV/IMPERFEKTIV)', wes $6 z$ / wesele / wesozy 'fröhlich (Kurzform)/Hochzeit/fröhlich; 
$\left\{\varepsilon_{5}\right\}$ für die überlappende Alternation $\{u\}$ vs. \{0\} vs. \{i\}, z.B. in boj / boju / bojowy / bic 'Kampf (NOM/GEN SG)/kämpferisch/schlagen' .

Für die Alternation $\left\{\&_{1}\right\}$ gilt Regel (PL17):

$$
\begin{aligned}
& (P L 17)(1)\{\& 1\} \rightarrow\{\tilde{e}\} /-\left\{\underline{C}_{1} \underline{V}_{1}\right\} \\
& \text { (2) }\left\{\&_{1}\right\} \rightarrow\{\tilde{e}\} /-\left\{\underline{C}_{1}, 3, \underline{V}\right\} \\
& \text { (3) }\left\{\varepsilon_{1}\right\} \rightarrow\{\tilde{e}\} /-\left\{\underline{C}_{1}, 3, \emptyset\right\} \\
& \text { (4) }\left\{\varepsilon_{1}\right\} \rightarrow\left\{\tilde{e}_{\}} /-\{\subseteq i\}\right.
\end{aligned}
$$

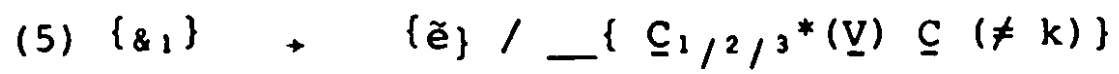

$$
\begin{aligned}
& \text { (6) }\left\{\varepsilon_{1}\right\} \rightarrow\left\{\tilde{e}_{\}} /-\left\{\underline{C}_{3} \emptyset_{\mathrm{FL}}\right\}\right. \\
& \text { (7) }\left\{\varepsilon_{1}\right\} \rightarrow\left\{\tilde{e}_{\}} /-\left\{\underline{C}_{3} \underline{V}_{F L}\right\}\right. \\
& \text { (8) }\left\{\varepsilon_{1}\right\} \rightarrow\{\tilde{o}\} /- \text { SONST }
\end{aligned}
$$

WENN : $\underline{c}_{1}:\left\{b ; b^{\prime} ; d ; 3 ; k ; g\right\}$

$$
\begin{aligned}
& \underline{C}_{2}:\{c\} \\
& \underline{C}_{3}:\left\{\mathrm{p} ; t_{i} \mathrm{~s} ; \mathrm{x}\right\} \\
& \underline{\mathrm{V}}_{1} \neq \text { FL }[+ \text { MASKULINUM }]
\end{aligned}
$$

Für die Alternation $\left\{\&_{2}\right\}$ gilt Regel (PL18):

$$
\begin{aligned}
(P L 18)(1)\left\{\&_{2}\right\} & \rightarrow\{e\} /\left(\left\{\underline{c}^{*},\right\}\right)-\{\underline{c}, \underline{V}\} \\
(2)\left\{\&_{2}\right\} & \rightarrow\{e\} /-\{\underline{c}, \emptyset\} \\
(3)\left\{\&_{2}\right\} & \rightarrow\{e\} /-\left\{\underline{c}_{1} *, s k\right\} \\
(4)\left\{\&_{2}\right\} & \rightarrow\{e\} /-\left\{\underline{c}_{1} *, n\right\} \\
(5)\left\{\&_{2}\right\} & \rightarrow\{e\} /-\left\{\underline{c}_{1} *, \dot{s}\right\} \\
(6)\left\{\&_{2}\right\} & \rightarrow\{a\} /- \text { SONST }
\end{aligned}
$$

WENN: $\quad \subseteq \quad \neq \quad \underline{C}_{1}:\{1 ; s ; t\}$

Für die Alternation $\left\{\&_{3}\right\}$ gilt Regel (PL19):

$$
\begin{aligned}
(P L 19)(1)\left\{\&_{3}\right\} & \rightarrow\{u\} /-\{\underline{c} \emptyset\} \\
(2)\left\{\&_{3}\right\} & \rightarrow\{u\} /-\{\underline{c}(8) \subseteq(\neq n)\} \\
(3)\left\{\&_{3}\right\} & \rightarrow\{u\} /-\left\{\underline{c}^{*} \emptyset\right\} \\
(4)\left\{\&_{3}\right\} & \rightarrow\{u\} /-\{j \emptyset\} \\
(5)\left\{\&_{3}\right\} & \rightarrow\{u\} /-\left\{v, \underline{v}_{1}\right\} \\
(6)\left\{\&_{3}\right\} & \rightarrow\{u\} /-\left\{v^{*}, n\right\} \\
(7)\left\{\&_{3}\right\} & \rightarrow\{0\} /- \text { SONST }
\end{aligned}
$$

WENN : $\subseteq$ : [+ STIMMHAFT]

$\underline{\mathrm{V}}_{1} \neq \mathrm{FL}$ 
Für die Alternation $\left\{\&_{4}\right\}$ gilt Regel (PL2O):

\begin{tabular}{|c|c|c|c|}
\hline$(P L 20)(1)$ & $\left\{\&_{4}\right\}$ & $\rightarrow$ & 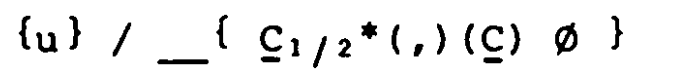 \\
\hline (2) & $\left\{\&_{4}\right\}$ & $\rightarrow$ & $\{u\} / \ldots\left\{\underline{c}_{1}, \underline{v}(\neq a)\right\}$ \\
\hline (3) & $\{\& 4\}$ & $\rightarrow$ & $\left.\{e\} /\left\{\underline{c}_{1}\right\}-\underline{c}_{2}(*)\right\}$ \\
\hline (4) & $\left\{\varepsilon_{4}\right\}$ & $\rightarrow$ & $\{e\} /\left\{c_{1}\right\}$ \\
\hline (5) & $\{\& 4\}$ & $\rightarrow$ & $\left.\underline{C}_{1},(\underline{C},) \emptyset\right)$ \\
\hline (6) & $\left\{8_{4}\right\}$ & + & $\{e\} /\{\underline{c}\}$, \\
\hline (7) & $\{\& 4\}$ & $\rightarrow$ & $\{a\} /-\left\{\subseteq_{1} \subseteq(),\right\}$ \\
\hline (8) & $\{\& 4\}$ & $\rightarrow$ & $\{a\} /-\left\{c_{1}, a\right\}$ \\
\hline (9) & $\left\{\&_{4}\right\}$ & $\rightarrow$ & $\{0\} /$ SONST \\
\hline
\end{tabular}

WENN : $\underline{c}_{1}:\{l ; r ; t ; d ; s ; z\}$

$\underline{C}_{2}:\{g\}$

Fưr die Alternation $\left\{\&_{s}\right\}$ gilt Regel (PL21):
$(P L 21)(1) \quad\{\& 5\} \rightarrow$
$\{u\} /-\{j(T M) K\}$
(2) $\left\{\&_{5}\right\} \rightarrow$
\{o\} $/\{j \underline{v}\}$
(3) $\left\{8_{5}\right\} \rightarrow$
\{i\}/
SONST

Der Obstruent $\left\{\mathrm{v}\left({ }^{\prime}\right)\right\}$ alterniert auf der phonetischen Ebene mit dem obstruenten [ $f(')]$ sowohl vor als auch hinter stimmlosen Obstruenten sowie im Auslaut. Für diese Alternation gilt:

(PT22)

$$
\left\{\mathrm{v}\left({ }^{\prime}\right)\right\} \rightarrow\left[f\left({ }^{\prime}\right)\right] /\left\{\begin{array}{l}
{[- \text { STIMMHAFT }]} \\
{[- \text { STIMMHAFT }]} \\
-[\#]
\end{array}\right\}
$$

Der Liquid $\{\check{r}\}$ alterniert auf der phonetischen Ebene mit dem Obstruenten [ $\dot{s}$ ] sowohl vor als auch hinter stimmlosen Obstruenten sowie in Wortauslaut. Für diese Alternation gilt:

(PT23)

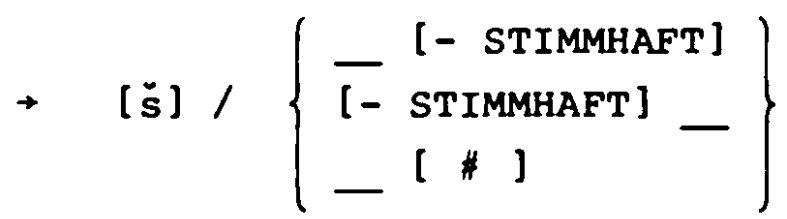

Beispiele für (PT22) und (PT23) siehe in $\S 2.1$ und $\S 2.3$.

Die Palatalisierung - ebenfalls auf der phonetischen Ebene - betrifft die Dentale $\{s\}$ und $\{z\}$, die vor palatalen Obstruenten und vor palatalen Nasalkonsonanten mit [s] bzw. [ $\xi$ ] alternieren, so z.B. in jazda / jezdzic 'Fahrt/fahren', oczywisty / oczywizcie 'selbstverständlich (A/ADV)', бsmy / osmiu 'der Achte/acht 
(GEN PL MASK)', jasny / jabnie 'hell/(in der Anrede) Hochwohlgeboren', spad / spioch 'schlafen/Schlafmütze'.

Fur diese Alternation gelten folgende PT-Regeln:

(PT24)

$$
[s] /=\left\{\begin{array}{c}
\varepsilon \\
p^{\prime} \\
s \\
f^{\prime} \\
f \\
h
\end{array}\right\}
$$

(PT25)

$$
\{z\}+[z] /-\left\{\begin{array}{l}
\xi \\
z
\end{array}\right\}
$$

In (PT24) und (PT25) handelt es sich um die Angleichung bezüglich Palatalitat, die zwar phonetisch motiviert ist, jedoch auf der morphonemischen Ebene ebenfalls ihre Funktion hat, so z.B. im Falle der Vokalalternation $a / e$, die entsprechend (PL18(1)) durch den Alternanten \{e\} dann realisiert wird, wenn sie vor einem palatalen Konsonanten steht. Wollte man für die morphonemische Ebene z.B. die Repräsentation $\left\{j \&_{2} z \xi i c\right\}$ und nicht $\left\{j \&_{2} z-\right.$ 3ic\} - für jezdzic 'fahren' - annehmen, so müBte diese Alternation durch $\{a\}$ realisiert werden, was *jazdzic ergabe.

Es konnte noch eine ganze Reihe von PT-Regeln aufgestellt werden, die die Angleichung bezüglich Palatalität zeigen würden: doch beschränken sich diese Angleichungen auf die phonetische Ebene, d.h., dab sie auf der morphonemischen Ebene keine entscheidende Funktion erfüllen: ${ }^{57}$ diese Angleichungen betreffen hauptsăchlich die Vokale, die durch die palatale Ungebung entweder nach vorn verlagert oder angehoben werden.

Es muB jedoch die Angleichung bezüglich [- PALATAL] berücksichtigt werden, nämlich für die Fälle, in denen bestimmte Auslautrepräsentationen von $R$, $T M$ und $K$ durch das Hinzufügen bestimmter $K$ - bzw. TM-Repräsentationen entpalatalisiert werden; es gibt nămlich einerseits: anioz - anielski 'Engel/Engels-', pan - parski 'Herr/Herren-', anderseits: chlop - chzopski 'Bauer/ Bauern-', brud - brudny 'Schmutz/schmutzig', wo das jeweils zweite Wort durch die Hinzufügung der $\mathrm{K}$-Repräsentationen $\{+*, \text { sk }+\}_{A}$ oder $\{+*,(8) n+\}_{A}$ gebildet wird. ${ }^{\text {so }}$ Im ersten Fall 
wird der Auslaut des Stames palatalisiert, in zweiten Fall dagegen nicht.

Für diese Nichtangleichung bezüglich Palatalitut bzw. für die Entpalatalisierung gilt folgende PL-Regel:

$(P L 26)\left\{C_{1}(),\right\}+\{(* /),(8) \subseteq\} \rightarrow\left\{C_{1} \subseteq\right.$ a $\}$

WENN: $\underline{c}_{1}:\left\{p_{i} b ; f ; v ; m ; t ; d ; r ; x\right\}$

UND: $0 \quad:$ Permutation von $n$ zeichen

Phonetisch motiviert ist schlieblich die Angleichung bezüglich der stimmhaftigkeit unter den Obstruenten; wie bel der Angleichung bezüglich der stimmlosigkeit müssen auch hier die Sonoranten ausgeschlossen werden, well sie sich bezüglich dieser Merkmale gegenüber den Obstruenten neutral verhalten; $d . h$. vor oder hinter einem Sonoranten bleibt ein stimmloser Obstruent stimmlos und ein stimmhafter stimmaft. Was die obstruenten vor bzw. hinter anderen Obstruenten betrifft, so gelten stimmaftigkeit vor stimmhaftigkeit und stimmlosigkeit vor stimmlosigkeit; im Wortauslaut gilt generell stimmlosigkeit.

Für die Obstruenten gilt also folgende PT-Regel: (PT27)

$$
\begin{aligned}
& {[ \pm \text { STIMMHAFT }] \rightarrow[- \text { STIMMHAFT }] /\left\{\begin{array}{l}
-[- \text { STIMMHAFT }] \\
{[\neq}
\end{array}\right\}} \\
& {[ \pm \text { STIMMHAFT }] \rightarrow[+ \text { STIMMHAFT }] /-[+ \text { STIMMHAFT }]}
\end{aligned}
$$

Beispiele für die stimmlosigkeit sind: [vutka] - wodka 'schnaps', [druk] - $d r \delta g$ ' (GEN PL) Weg', Beispiele für die Stimmhaftigkeit sind: [podbuj] - podboj 'Exoberung', [oddat] - oddac 'abgeben', [pod domem] - pod domem 'unter dem Haus'. Das zuletzt genannte Beispiel zeigt, das die Angleichung bezüglich der stimmaftigkeit auch uber die Grenzen des Worts hinausgeht; dies bezleht sich jedoch nur auf die prăposition, die mit dem ihr zugeordneten Wort im Polnischen eine phonetische Einheit bildet. Diese Feststellung labt sich deutlich anhand von Verbindungen von $P$ mit einem einsilbigen Wort zeigen, wo der Penultima-Akzent des Polnischen auf die $P$ und nicht auf das wort gesetzt wird, so z.B. in [u nas] - $u$ nas 'bel uns' oder [zà fim] - $z a$ nim 'hinter ihn'. 


\subsubsection{MORPHONOLOGISCHE TILGUNGSREGELN}

Das Hinzufügen bestimmter $K$-Repräsentationen hat nicht nur den Morphonemwechsel, sondern auch Tilgungen zur Folge. Diese Tilgungen werden ebenfalls in Regeln gefaBt, die DL-Regeln genannt und fortlaufend im AnschluB an die PR-, PT- und PL-Regeln - vgl. $\S 7.1 .2$ - numeriert werden.

Für die Bildung der Adjektive vom Typ praski 'Prager', francus$k i$ 'französisch', wZoski 'italienisch', grecki 'griechisch', die von den Nomina Praga 'Prag', Francuz 'Franzose', WZoch 'Italiener', Grek 'Grieche' gebildet werden, gelten folgende DL-Regeln:

\begin{tabular}{|c|c|c|c|c|c|c|c|}
\hline \multirow[t]{8}{*}{ (DL28) } & $\{\dot{c}\}$ & + & $\{\mathbf{s}\}$ & $\rightarrow$ & $\{\check{c} s\}$ & + & $\{c\}$ \\
\hline & $\{\dot{s}\}$ & + & $\{\mathbf{s}\}$ & $\rightarrow$ & $\{\check{s} s\}$ & $\rightarrow$ & $\{s\}$ \\
\hline & $\{\bar{z}\}$ & + & $\{s\}$ & $\rightarrow$ & $\{\check{z} \mathbf{s}\}$ & $\rightarrow$ & $\{s\}$ \\
\hline & $\{\dot{\mathbf{r}}\}$ & + & $\{s\}$ & $\rightarrow$ & $\{\check{r} s\}$ & $\rightarrow$ & $\{r s\}$ \\
\hline & $\{\dot{3}\}$ & + & $\{s\}$ & $\rightarrow$ & $\{\dot{3 s}\}$ & $\rightarrow$ & $\{3\}$ \\
\hline & $\{c \hat{c}\}$ & + & $\{s\}$ & $\rightarrow$ & $\{\dot{c} s\}$ & $\rightarrow$ & $\{c\}$ \\
\hline & $\{z\}$ & + & $\{s\}$ & + & $\{\varepsilon s\}$ & + & $\{s\}$ \\
\hline & $\{\dot{s}\}$ & + & $\{s\}$ & $\rightarrow$ & $\{$ śs $\}$ & $\rightarrow$ & $\{s\}$ \\
\hline
\end{tabular}

Die Anwendung der Regel (DL28) setzt die Anwendung der Regeln (PL8) und (PL15) voraus; dies entsprechend "Stern" und "Komma", die an der vorderen Grenze der K-Repräsentation notiert sind. Für die Anwendung von (DL28) zwei Bejspiele:

$$
\text { (71) } \begin{aligned}
& \{- \text { prag }+\emptyset+\}_{\mathrm{N}}+\left\{+^{*}, \mathrm{sk}_{\mathrm{A}} \rightarrow(\}_{\mathrm{A}} \rightarrow \rightarrow\right. \\
\rightarrow & \{- \text { pražsk }+\}_{\mathrm{A}} \rightarrow(\mathrm{DL} 28) \rightarrow \\
\rightarrow & \{- \text { prask }+\}_{\mathrm{A}}+\{+\mathrm{i} \#\}_{\mathrm{FL}} \text { praski } \\
& \{- \text { vlox }+\emptyset\}_{\mathrm{N}}+\left\{+^{*}, \mathrm{sk}+\right\}_{\mathrm{A}} \rightarrow(\mathrm{PL} 8) \rightarrow \\
\rightarrow & \{- \text { vlos }, \mathrm{sk}+\}_{\mathrm{A}} \rightarrow(\mathrm{PL} 13) \rightarrow \\
\rightarrow & \{- \text { vlossk }+\}_{\mathrm{A}} \rightarrow(\mathrm{DL} 28) \rightarrow \\
\rightarrow & \{- \text { vlosk }+\}_{\mathrm{A}}+\{+i \#\}_{\mathrm{FL}} \text { wzoski }
\end{aligned}
$$

Im zweiten Beispiel in (71) kommt also noch Regel (PL13) als Voraussetzung hinzu.

Bei der Realisierung des Alternanten muB also eine bestimmte Reihenfolge beachtet werden, nämlich "Stern" vor "Komma"; denn zunächst muß der Alternant $\{x\} \operatorname{durch}\{\check{s}\}$, anschließend $\{\dot{s}\}$ durch $\{\dot{s}\}$ ersetzt werden, d.h. (PL8) vor (PL13). 
7.1.3.1 DER MORPHONOLOGISCHE OPERATOR $Q$

In Anlehnung an Jakobson (1944) nimmt Isačenko $(1969,63)$ einen nicht spezifizierten Konsonanten 2 an. Jakobson führt $Q$ u.a. deshalb ein, um $z u$ vermeiden, daB ein vokalisch auslautender Wortstamm, dem ein vokalisch anlautendes Infix oder Suffix hinzugefügt wird, eine doppelte Vokalrepräsentation zur Folge hat. Dafür ein Beispiel aus dem Polnischen, wenn davon ausgegangen wird, daß das Nomen caytanie 'Lesen' von caytac 'lesen' gebildet wird (vgl. auch (56)):

$$
\begin{aligned}
& \{-\ddot{c i t a}+a+\}_{V}+\{+a \hat{f}+\}_{N} \rightarrow \\
& \rightarrow\{-\check{c i t a}+a+Q+a f+\}_{N} \rightarrow \\
& \rightarrow\{-\check{c i t a q a h}+\}_{\mathrm{N}} \\
& \rightarrow\{-\check{c i t a h}+\}_{N}+\{+e \#\}_{F L} \\
& \text { caytanie }
\end{aligned}
$$

Die Einsetzung von $Q$ in (72) entspricht der von Jakobson aufgestellten Regel:

$$
\underline{v}_{1}+\underline{v}_{2}+\underline{v}_{2}
$$

Regel (73) entspricht - ebenso wie TF, vgl. $\$ 6.2 .1 .2$ - der Feststellung, daB der Auslaut des zu suffigierenden Wortstames konsonantisch sein muB; deshalb wird $Q$ auch in einigen Fällen als $\{j\}$ realisiert, so bei Isačenko (1974) in den russischen Nominalstämmen. Im Polnischen trägt dieser Feststellung selbst die graphemische Repräsentation Rechnung, so z.B. in religijny 'religiös' von religia 'Religion'.

Isačenko (1969) stellt eine zweite Regel auf, nämlich:

$$
\underline{\mathrm{V}}_{1}+\underline{\mathrm{V}}_{2}+\underline{\mathrm{V}}_{1}
$$

Bezogen auf den nicht spezifizierten Konsonanten $Q$ entspricht Regel (74) folgenden Voraussetzungen:

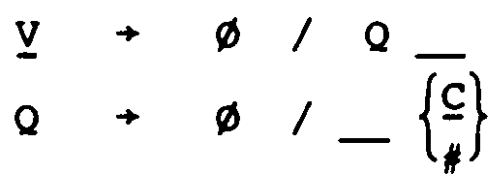

Die Aufhebung von $Q$ sowohl in der stellung vor einem Konsonanten als auch am Wortende liegt u.a. darin begründet, daB Isačenko $Q$ auch für die nicht flektierenden wörter im Russischen, die auf einen Vokal auslauten, annimmt. 
Als Folgerung aus (73), (74) und (75) kann folgende DL-Regel aufgestellt werden:

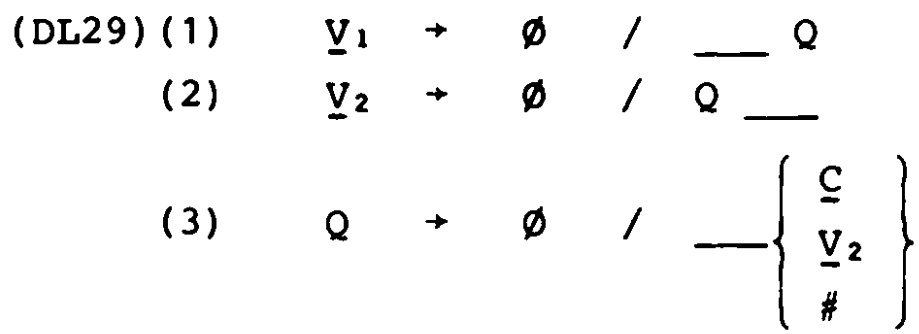

Regel (DL29) läBt den ersten Vokal tilgen, wenn dieser vor $Q$ steht; steht der Vokal hinter $Q$, so wird er der zweiten Regel entsprechend getilgt. Wollte man also beide Regeln, also (DL29 (1)) und (DL29(2)) anwenden, so müBten beide Vokale getilgt werden. Doch hier entscheiden die Morphemgrenzen darüber, wo $Q$ einzusetzen ist und welcher Vokal entsprechend dieser Einsetzung getilgt wird. In (76) z.B. wird (DL29(1)) angewandt, um die morphonemische Repräsentation des verbalen $T M z u$ tilgen; $Q$ wird also dem TM zugefügt und nicht $K$, nämlich:

$$
l_{\mathrm{N}}[\mathrm{v}=\mathrm{vi}-\text { kon }+\mathrm{a}+\mathrm{Q}]+\mathrm{a}+\mathrm{]} \text { wykonanie 'Ausführung' }
$$

Die Feststellung, daB bei der Nominalisierung von Verbalstämmen nicht der Vokal des $K$, sondern der Vokal des verbalen TM, also - der Jakobsonschen Regel (73) entsprechend - $\underline{V}_{1}$ getilgt wird, läßt sich durch die Nominalisierung anderer Verbklassen deutlicher machen, so z.B. im Falle der Bildung von mowienie von mowic oder von postanowienie von postanowic 'Reden-reden, BeschluB-beschließen' :

$$
\begin{aligned}
& \left.l_{N}\left[l_{V}=-8_{3} v^{\prime}+i+Q\right]+, e h+\right] \\
& {\left[{ }_{N}\left[l_{v}=\text { po-stanov } v^{\prime}+i+Q\right]+, e h+\right]}
\end{aligned}
$$

Würde in (77) nicht die morphonemische Repräsentation von TM, sondern von $\mathrm{K}$ getilgt, also würde hier die Regel (74) von Isačenko angewandt, dann müBte es heiBen *mowinie und *postanowinie.

Mit Hilfe von $Q$ wird also verhindert, daB $\underline{V}$ - Verbindungen in der $W$-Basis bei Hinzufügung einer entsprechenden $\mathrm{K}$-Repräsentation entstehen; daher nenne ich $Q$ einen morphonologischen operator.

Als morphonologischer Operator kann $Q$ eine zusätzliche Funktion 
übernehmen, nämlich die generelle Kennzeichnung der hinteren Grenze des Wortstammes, dem ein K zugefügt wird. Obgleich die Hinzufügung von $Q$ in (76) und (77) die Tilgung des vokalischen Auslauts zur Folge hat, bleibt die Assoziation mit den inhärenten Merkmalen des Verbalstammes erhalten. Demnach tritt an die Stelle eines $K$, der entsprechend (61) nur an letzter stelle des Wortbildungsteils stehen kann, der verbale TM. Dies gilt auch für nominale Wortstämme, in denen kein vokalischer Auslaut getilgt wird und die trotz Nominalisierung mit ihren inhärenten Merkmalen assoziiert werden, so z.B. im Falle von kioskarz 'Kioskhändler', dem der Nominalstamm kiosk mit dem nominalen "Null"-Klassifikator zugrunde liegt sowie die K-Repräsentation $\{+a \bar{r}+\}_{N}$. Der Funktionswechsel zwischen $K$ und $T M-v g l$. $\S$ 6.2.1.3 - wird also von $Q$ angezeigt, und es gilt die DL-Regel: (DL30) $K+Q$ TM / K

Regel (DL30) besagt, daß K die Funktion eines TM dann übernimmt, wenn ein $K$ hinzugefügt wird. (DL 30) gilt auch dann, wenn mehrere TM-Repräsentationen in der $W$-Basis vor $K$ stehen.

Der morphonologische Operator $Q$ hat also einerseits eine phonologische Funktion, indem er verhindert, daß vokalische Dubletten entstehen, anderseits aber eine morphosyntaktische Funktion, die darin beruht, daß er einem Klassifikator, der vor einem anderen Klassifikator stellung bezieht, die Funktion eines stamm-Markers zuweist; damit bleibt die Assoziation mit den inhärenten Merkmalen des Wortstammes erhalten, so im Falle der Beispiele (76) und (77) mit den Merkmalen, zu denen die vom Verbalstamm bezeichnete 'Tätigkeit' gehört.

\subsection{MERKMALE}

Der Basis jedes zu bildenden Worts liegen Merkmale zugrunde. So werden die Segmente des Morphems, d.i. die Morphoneme, durch phonologische Merkmale gekennzeichnet, die dem Morphonem-Inventar (68) entnommen werden. Die Morpheme sind sowohl durch die Grenzen-Merkmale (13) als auch durch die Morphem-Marker (61) gekennzeichnet. Da die wortbildung in der Satz-Basis - vgl. \$3.1stattfindet, sind die Morphem-Marker u.a. mit der lexikalischen Kategorie verbunden; dies bezieht sich auf $K$ und TM. Die Mor- 
phem-Marker $K$ und $T M$ sind also durch jeweils ein lexikalisches Kategorienmerkmal gekennzeichnet.

Die Kategorienmerkmale implizieren im Polnischen eine ganze Reihe von welteren Kategorienmerkmalen sowie von inhärenten Merkmalen, die allesant zum Merkmalkomplex gehören, der jeder W-Basis zugrunde liegt. Dazu zăhlen sowohl die Merkmale (16), (20) und teilweise (22) als auch die Merkmale der morphonemischen Realisierung der Morphem-Marker PF und IF. Schlieblich impliziert das Kategorienmerkmal die Kategorie des Genus.

Die für die Nominalisierung im Polnischen notwendigen Merkmale, die nicht $z$ den phonologischen sowie $z u$ den morphosyntaktischen Merkmalen zählen, werden in Kategorienmerkmale und inhärente Merkmale unterteilt.

Zu den Kategorienmerkmalen im Polnischen gehören:

(78) (a) die lexikalischen Kategorien N, V, A, ADV, PRO,...;

(b) die Kategorie des Genus;

(c) die Kategorie des Aspekts und des Genus Verbi.

Zu den inhärenten Merkmalen im Polnischen gehören die Merkmale (16), die um einige weitere Merkmale in (92) und (94) ergănzt werden müssen.

\subsubsection{LEXIKALISCHE KATEGORIE}

Für die lexikalische Kategorie gilt die Behauptung, daß sie Element eines systems ist, das auf dem prinzip der Binarität beruht. Schmid $(1970,10 \mathrm{f}$.$) stellt ein solches wortarten-System$ auf, das von der zeichendefinition Saussures sowie von der zeichentheorie Morris' ausgeht und sich auf folgende Forderung stutzt:

(79) Jedes sprachliche zeichen muB in seinen Relationen a) zu anderen Zeichen, b) zu den Objekten, die es bezeichnet, und c) zum sprecher bzw. zur Sprechsituation bestimut werden.

Demzufolge wird die lexikalische Kategorie bezüglich der semantischen Komponente [ \pm SEM], der syntaktischen [ $\pm S Y N]$, der pragmatischen [ \pm PRAG] sowie der Komponente der Autonomie [ \pm AUT] einerseits, anderseits bezüglich ihrer stellung gegen- 
uber einer anderen lexikalischen Kategorie sowie der wort- und Satzpause definiert; dabei gehört die pragmatische Komponente, z.B. die Sprechsituation, zur Sprachkompetenz.

Zur Veranschaulichung dieser Relationen entnehme ich diesem System N, V, A, ADV sowie die Wortpause (WP):

$\begin{array}{rcccc} & \text { SEM } & \text { SYN } & \text { PRAG } & \text { AUT } \\ \text { N } & + & + & - & + \\ \mathrm{V} & + & + & + & + \\ \mathrm{A} & + & + & + & - \\ \mathrm{ADV} & + & - & + & - \\ \mathrm{WP} & - & - & - & -\end{array}$

Der Schmidschen Klassifizierung (80) entsprechend gelten folgende Definitionen:

$$
\begin{aligned}
& N \rightarrow\left[\begin{array}{l}
+ \text { SEM } \\
+ \text { SYN } \\
- \text { PRAG } \\
+ \text { AUT }
\end{array}\right] \quad V \rightarrow\left[\begin{array}{l}
+ \text { SEM } \\
+ \text { SYN } \\
+ \text { PRAG } \\
+ \text { AUT }
\end{array}\right] \quad A \rightarrow\left[\begin{array}{l}
+ \text { SEM } \\
+ \text { SYN } \\
+ \text { PRAG } \\
- \text { AUT }
\end{array}\right] \\
& \text { ADV } \rightarrow\left[\begin{array}{l}
+ \text { SEM } \\
- \text { SYN } \\
+ \text { PRAG } \\
- \text { AUT }
\end{array}\right] \quad \text { WP } \rightarrow\left[\begin{array}{l}
- \text { SEM } \\
- \text { SYN } \\
- \text { PRAG } \\
- \text { AUT }
\end{array}\right]
\end{aligned}
$$

Ausgehend von den Grundlagen, die Chomsky (1970) geschaffen hat, geht man in der Erweiterten Standard-Theorie von einer wesentlich einfacheren Definition, allerdings nur der drei bzw. vier lexikalischen Hauptkategorien aus. Den Ausgang bilden die Relationen:

\begin{tabular}{c|c|c} 
& $+\mathrm{N}$ & $-\mathrm{N}$ \\
\hline$+\mathrm{V}$ & $\mathrm{A}$ & $\mathrm{V}$ \\
\hline$-\mathrm{V}$ & $\mathrm{N}$ &
\end{tabular}

In (82) werden die lexikalischen Hauptkategorien also mittels der Merkmale $[ \pm N]$ sowie $[ \pm V]$ definiert. Die leere Stelle wird den Erfordernissen der Syntax entsprechend durch P - vgl. u.a. Jackendoff (1977,31) - ersetzt; und es gelten - vgl. Radford $(1981,110)$ - folgende Definitionen für die lexikalischen Hauptkategorien $N, V, A$ und $P$ : 


$$
N \rightarrow\left[\begin{array}{l}
-V \\
+N
\end{array}\right] \quad V \rightarrow\left[\begin{array}{l}
+V \\
-N
\end{array}\right] \quad A \rightarrow\left[\begin{array}{l}
+V \\
+N
\end{array}\right] \quad P \rightarrow\left[\begin{array}{l}
-V \\
-N
\end{array}\right]
$$

Das Pronomen wird als pronominaler Ausdruck in Relation zum nichtpronominalen Ausdruck gesetzt; das gleiche gilt für das Adverb. Für PRO und ADV gelten folglich die Definitionen:

$$
\mathrm{PRO} \rightarrow\left[\begin{array}{l}
-\mathrm{V} \\
+\mathrm{N} \\
+\mathrm{PRO}
\end{array}\right] \quad \mathrm{ADV} \rightarrow\left[\begin{array}{l}
+\mathrm{V} \\
+\mathrm{N} \\
+\mathrm{ADV}
\end{array}\right]
$$

Aus den Definitionen (84) läBt sich schlieben, daB das Merkmal [- PRO] von der Definition des $N$ in (83) impliziert wird, wăhrend die Definition des $A$ in (83) das Merkmal [- ADV] impliziert. 59

\subsubsection{GENUS UND GESCHLECHT}

Im Polnischen wird zwischen den drei Genera, zwischen MASKULINUM, FEMININUM und NEUTRUM unterschieden. Den drei Genera, bzw. den drei grammatischen Geschlechtern entsprechen auch drei Deklinationen. Deshalb u.a. ist es in der Wortbildung von Bedeutung, dem zu bildenden wort auch das entsprechende grammatische Geschlecht zuzuordnen.

Nach Jakobson $\left(1932,10 f_{.}\right)$- vgl. auch $\S 2.1$ - ist das NEUTRUM gegenüber dem FEMININUM und dem MASKULINUM merkmalhaft bzw. markiert, während das FEMININUM gegenüber dem MASKULINUM markiert ist. Dies gilt bei Jakobson für das Russische; doch ist es im Polnischen nicht anders.

Für die Markiertheit vs. Unmarkiertheit der drei Genera im Polnischen können folgende Relationen aufgestellt werden:

\begin{tabular}{r|c|c|c|} 
& NEUTRUM & FEMININUM & MASKULINUM \\
\hline NEUTRUM & $u$ & $u$ & $u$ \\
FEMININUM & $m$ & $u$ & $u$ \\
MASKULINUM & $m$ & $m$ & $u$
\end{tabular}

Demnach ist NEUTRUM das am stärksten, denn gegenüber FEMININUM und MASKULINUM markierte Genus, während FEMININUM nur gegenüber MASKULINUM markiert ist, MASKULINUM dagegen bezüglich NEUTRUM und FEMININUM unmarkiert ist. 
Die Unmarkiertheit des Genus MASKULINUM wird auch in der wortbildung deutlich, so u.a. in der Bildung demaskuliner Feminina, wie z.B. Lekarz - Zekarka 'Arzt-Äztin' oder gornik-gorniozka 'Bergmann-Bergmännin', während die Markiertheit des Genus NEUTRUM gegenüber dem Genus MASKULINUM sich in den wörtern lekarstwo und gornictwo 'Arznei, Bergbau(wesen)' zeigt; denn in diesen wörtern bleiben die maskulinen TM-Repräsentationen, nämlich die morphonemischen Entsprechungen von -arz- und - $n i k$ - erhalten.

Die Unterscheidung zwischen dem grammatischen und dem natürlichen Geschlecht ist für solche Feminina im Polnischen von Bedeutung, die zwar in der Subkategorisierung wie Maskulina behandelt werden, jedoch in der Flexion - auBer im NOM PL - zu den Feminina gezählt werden müssen. Dazu gehören wörter vom Typ meźczyzna 'Mann', poeta 'Dichter'. Diese wörter unterscheiden sich in der Flexion von den Feminina allein durch den Stammauslautwechsel im NOM PL; hier heiBt es nämlich mezczyźni, poeci aber kobiety 'Frauen', d.h., daß, im Gegensatz zu kobiety, der konsonantische stammauslaut mit dem entsprechend durch "Stern" und/oder "Komma" gekennzeichneten Alternanten korreliert. 60

Für diese wörter können folgende Merkmale angenommen werden:

$$
\left[\begin{array}{l}
+ \text { FEMININUM } \\
+ \text { MENSCHLICH } \\
+ \text { MANNLICH }
\end{array}\right]
$$

Demnach ist das Genus FEMININUM nicht nur gegenüber MASKULINUM, sondern auch gegenüber [+ MÄNNLICH], bei Implikation des Merkmals [+ MENSCHLICH], markiert.

Das Merkmal des natürlichen Geschlechts [+ MÄNLICH] hat nichts mit der auBersprachlichen wirklichkeit $z u$ tun; es betrifft einzig und allein die valenz, die das $z u$ bildende Nomen bezüglich u.a. der Adjektivendung oder der Verbendung hat. Dieses Merkmal verhindert z.B., daB verbindungen wie z.B. *wysoka mezczyzna powiedziaza 'eine große Mann sagte' oder *wybitna poeta czytaza 'eine geniale Dichter las' entstehen, statt wysoki meiczyzna powiedziaz 'ein großer Mann sagte' sowie wybitny poeta czytaz 'ein genialer Dichter las'. AuBer der Valenz betrifft das Merkmal [+ MANNLICH] den Konsonantenwechsel im NOM PL. 
Die drei Genusmerkmale im Polnischen lassen sich binăr folgendermaken definieren:

$$
\begin{array}{lll}
{[+ \text { NEUTRUM }]} & \rightarrow & {\left[\begin{array}{l}
- \text { FEMININUM } \\
- \text { MASKULINUM }
\end{array}\right]} \\
{[+ \text { FEMININUM }]} & \rightarrow & {\left[\begin{array}{l}
+ \text { FEMININUM } \\
- \text { MASKULINUM }
\end{array}\right]} \\
{[+ \text { MASKULINUM }]} & \rightarrow & {\left[\begin{array}{l}
- \text { FEMININUM } \\
+ \text { MASKULINUM }
\end{array}\right]}
\end{array}
$$

Im Falle der "Maskulina" mit femininer Deklination wird I+ FEMININUM] durch [+ MÄNNLICH] ergänzt.

\subsubsection{ASPEKT UND GENUS VERBI}

Aspekt und Genus Verbi gehören $z u$ den verbinhärenten Merkmalen. Da das Verb nominalisiert wird, werden diese Merkmale den nomeninhärenten Merkmalen vorweggeschickt.

In Anlehnung an Jakobson $\left(1932,6 f_{.}\right)$sowie an die Diskussion der Aktionsarten im Russischen von Isačenko $(1975, \S 202-\$ 220)$ werden für die Aspekt- und Genus-Verbi-Korrelation im Polnischen folgende Merkmale angenommen:

$$
\begin{aligned}
& \text { V } \\
& \text { [ - GENUS VERBI] } \\
& \text { [+ ASPEKT ] } \\
& \text { [ + PERFEKTIV] } \\
& \text { [ - IMPERFEKTIV] } \\
& \text { [ - DELIMITATIV] } \\
& \text { [ - PERFEKTIV] } \\
& \text { [+ IMPERFEKTIV] } \\
& \text { [ - DURATIV] } \\
& \text { [ - AUGMENTATIV] } \\
& \text { [+ GENUS VERBI] } \\
& \text { [+ INTRANSITIV] } \\
& \text { [ - INTRANSITIV] } \\
& \text { [ - PASSIV] } \\
& \begin{array}{ll}
\rightarrow & {[ \pm \text { ASPEKT; } \pm \text { GENUS VERBI }]} \\
\rightarrow & {[ \pm \text { ASPEKT }]} \\
\rightarrow & {[ \pm \text { PERFEKTIV }]} \\
\rightarrow & {[- \text { IMPERFEKTIV }]} \\
\rightarrow & {[ \pm \text { DELIMITATIV }]} \\
\rightarrow & {[ \pm \text { SEMELFAKTIV }]} \\
\rightarrow & {[ \pm \text { IMPERFEKTIV }]} \\
\rightarrow & {[ \pm \text { DURATIV }]} \\
\rightarrow & {[ \pm \text { AUGMENTATIV }]} \\
\rightarrow & {[ \pm \text { KOMPLETIV }]} \\
\rightarrow & {[ \pm \text { INTRANSITIV }]} \\
\rightarrow & {[ \pm \text { PASSIV }]} \\
\rightarrow & {[ \pm \text { AKTIV }]} \\
\rightarrow & {[ \pm \text { REFLEXIV }]}
\end{array}
\end{aligned}
$$

In (88) wird also $V$ durch den Aspekt und das Genus Verbi ersetzt, um anschlieBend die Aspektmerkmale in Relation zu den Merkmalen der Aktionsarten und zu den Merkmalen des Genus Verbi zu setzen. 


\subsubsection{DIE INHÄRENTEN MERKMALE}

Die inhärenten Merkmale, die in diesem Abschnitt aufgestellt werden sollen, liegen sowohl dem $2 u$ bildenden wort als auch dessen Ursprung und damit den lexikalischen Kategorien N, V, A zugrunde.

Die natürliche, d.h. die nichtkonstruierte Paraphrase bzw. Wortverbindung, die der Sprecher des Polnischen z.B. mit dem Nomen agentis spawacz 'SchweiBer' als bedeutungsgleich assozilert, lautet:

$$
\begin{aligned}
& \text { czZowiek, co spawa /zawodowo/ } \\
& \text { 'ein Mensch, der (beruflich) schweiBt' }
\end{aligned}
$$

Es gehört zu den Eigenarten auch der Wortbildung im Polnischen, daB der Sprecher keiner solchen Paraphrase im RedefluB bedarf, um das objekt 'SchweiBer' zu bezeichnen; es reicht, wenn er [spavač] sagt. Mit diesem wort assoziiert er sowohl die vom Verbalstamm bezeichnete 'Tätigkeit' des 'SchweiBens' als auch den vom Klassifikator bezeichneten 'Träger' dieser Tätigkeit. Damit wird diesem Wort eine $F$ u $k t$ i o $n$ zuteil, die der von ihm bezeichneten 'Tätigkeit' entspricht und die dem 'Träger' zugeordnet wird. DaB es sich bei dieser 'Tätigkeit' um eine 'berufliche' handelt, wird als Denotatum gesehen und kann in einer entsprechenden Regel als Merkmal notiert werden.

Das bestimmte, belebte, menschliche objekt also ist Träger der durch die Tätigkeit bestimmten Funktion. Während der Stamm-Marker durch $\mathrm{V}$ gekennzeichnet ist, das die Tätigkeit bezeichnet, wird der Klassifikator durch $\mathbf{N}$ gekennzeichnet, dem - entsprechend (16) - u.a. die Merkmale [+ BELEBT; + MENSCHLICH] zugeordnet werden. Dem zu bildenden Nomen spawacz liegt also bezüglich der durch die Tätigkeit bestimmten Funktion das Merkmal [+ TRÄGER] zugrunde: und es gilt folgende Behauptung:

(90) Das Merkmal [+ TRAGER] kennzeichnet das Appellativum - vgl. (16) - als ein objekt, dem eine bestimmte Tätigkeit oder eine bestimmte Eigenschaft oder aber ein bestimmter Zustand - alle drei im Sinne der Definitionen (95) - zugeordnet werden.

Um das Merkmal [ \pm TRAGER] - wie es in (90) klassifiziert ist - 
- werden die Merkmale (16) in (92) erweitert.

Im Nomen spawalnia 'SchweiBerei' liegt ebenfalls die Funktion bezüglich des 'SchweiBens' vor, die jedoch keinem belebten, sondern einem nicht belebten $N$ zugeordnet wird. Damit liegt auch dem nicht belebten Nomen das Merkmal [+ TRÄGER] zugrunde, in diesem Fall im Sinne von [ \pm RAUMLICH]; und es gilt folgende Behauptung:

(91) Das Merkmal [ \pm RÄUMLICH] entspricht der Dimensionalität, die dem Appellativum sowohl im Sinne von [+ TRAGER] als auch im Sinne von [- TRAGER] zugeordnet werden kann. Das Merkmal [+ RAUMLICH] kann durch [ \pm DREIDIMENSIONAL] ersetzt werden. ${ }^{61}$

Durch das Merkmal [+ TRAGER] wird also hier der häufig benutzte Agens ersetzt; dies u.a. aus zwei Gründen: Zum einen ist der Agens - im Gegensatz zum Patiens - besser in der syntax aufgehoben, wo es um Subjekt-objekt-Beziehungen geht, zum anderen wäre es nicht ganz schlüssig, von einem nicht belebten Agens auszugehen.

Entsprechend den Behauptungen (90) und (91) werden die nomeninhärenten Merkmale (16) durch die nomeninhärenten Merkmale (92) ersetzt.

\begin{tabular}{|c|c|c|}
\hline $\mathbf{N}$ & $\rightarrow$ & [ \pm APPELLATIVUM] \\
\hline [ + APPELLATIVUM] & $\rightarrow$ & [ \pm TRAIGER $]$ \\
\hline \pm TRAGER & $\rightarrow$ & [ \pm INDIVIDUATIVUM] \\
\hline - INDIVIDUATIVUM] & $\rightarrow$ & [ \pm ABSTRAKTUM ] \\
\hline + INDIVIDUATIVUM] & $\rightarrow$ & {$[ \pm$ BELEBT $]$} \\
\hline + BELEBT ] & $\rightarrow$ & [ \pm MENSCHLICH ] \\
\hline - BELEBT ] & $\rightarrow$ & [ \pm RÄUMLICH] \\
\hline RAUMLICH] & $\rightarrow$ & [ \pm DREIDIMENSIONAL] \\
\hline
\end{tabular}

Uber das Merkmal $N$ sowie [ \pm TRAGER] wird für das Polnische ein zusătzliches binäres system inhärenter Merkmale in (92) einbezogen, wenn folgende Behauptung gilt:

(93) Wenn das Appellativum durch einen Träger ersetzt werden kann, dem - entsprechend (90) - eine bestimmte Tätigkeit oder ein bestimmter Zustand oder aber eine bestimmte Eigenschaft zugeordnet werden können, dies im sinne einer 
Verursachung oder einer Veranlassung, dann setzt diese Ersetzung voraus, daB diesem Appellativum eine Funktion zugrunde liegt.

Die Funktion des Nomen spawacz ist also das Tragen einer Tátigkelt im Sinne einer Festlegung bezüglich dieser Funktion, wăhrend das Nomen spawalnia ebenfalls die Funktion des Tragens der vom verbalen stamm bezeichneten Tatigkeit beinhaltet, diese jedoch im sinne eines festgelegtseins bezüglich dieser Funktion. Im Hinblick auf das Merkmal [ \pm TRAGER] in (92) und infolge der Behauptung (93) werden folgende Merkmale 62 unterschieden:

\begin{tabular}{|c|c|c|}
\hline $\mathbf{N}$ & $\rightarrow$ & [ \pm FUNRTION $]$ \\
\hline$[+$ FUNKTION $]$ & $\rightarrow$ & [ \pm BESTIMMTSEIN] \\
\hline [+ BESTIMMTSEIN] & $\rightarrow$ & [ \pm EIGENSCHAFT ] \\
\hline [ - EIGENSCHAFT ] & $\rightarrow$ & [ \pm ZUGEHठRIGKEIT $]$ \\
\hline [ - ZUGEHठRIGKEIT ] & $\rightarrow$ & [ \pm INSTRUMENTALITÄT] \\
\hline [+ INSTRUMENTALITAT] & $\rightarrow$ & {$[ \pm$ MASCHINE] } \\
\hline [ - MASCHINE $]$ & $\rightarrow$ & [ \pm MITTEL $]$ \\
\hline [- BESTIMMTSEIN] & $\rightarrow$ & [ \pm BESTIMMUNG ] \\
\hline [ + BESTIMMUNG ] & $\rightarrow$ & [ \pm ZUSTAND] \\
\hline [ - ZUSTAND ] & $\rightarrow$ & [ \pm TÄTIGKEIT] \\
\hline
\end{tabular}

Die Merkmale in (94) werden folgendermaBen definiert:

(95) [+ FUNKTION] entspricht einer Verursachung (kausal) oder einer Veranlassung (final) für

[+ BESTIMMTSEIN] entspricht einem Festgelegtsein - mit abgeschlossenem charakter - eines physischen oder nichtphysischen Objekts bezüglich einer Eigenschaft oder einer Zugehörigkeit oder einer Instrumentalităt. Bestimmtsein steht im Gegensatz zu

[+ BESTIMMUNG] entspricht einer Festlegung - mit offenem Charakter - eines physischen oder eines nichtphysischen objekts bezüglich eines anderen physischen oder nichtphysischen objekts oder/und bezulglich zustand oder Tätigkeit.

[+ zUSTAND] entspricht einer Fixierung - im Sinne von Festlegung - welcher auch immer Art bezüglich Raum oder/ /und zeit sowie bezüglich qualităt oder/und quantitat. 
[+ TATIGKEIT] entspricht einer Veränderung welcher auch immer Art bezüglich Raum oder/und zeit sowie bezüglich Qualität oder/und Quantität.

[+ EIGENSCHAFT] entspricht einem Festgelegtsein bezüglich Raum oder/und zeit sowie bezüglich Qualität oder/ /und Quantität.

[+ zUGEHORIGKEIT] entspricht einem Festgelegtsein bezüglich eines physischen oder nichtphysischen objekts.

[+ INSTRUMENTALITÄT] entspricht einem Festgelegtsein bezüglich eines Attributs im Sinne eines Teils von Funktion.

Demnach entspricht das Nomen spawacz einer Festlegung des Trägers, der belebt ist, bezulglich der vom Verbalstamm bezeichneten Tätigkeit, das Nomen spawalnia dagegen entspricht einem Festgelegtsein des unbelebten, räumlichen Trägers bezüglich der vom Verbalstamm bezeichneten Tätigkeit, also entspricht dieses Nomen einem Festgelegtsein bezüglich Instrumentalität.

\subsection{DIE WORTBILDUNGSREGELN}

Die Wortbildungsregeln sind Bestandteil des Lexikons. Die Wortbildungsregeln zeigen die Bildung des Worts anhand der kategorialen und der inhärenten Merkmale sowie anhand der morphosyntaktischen struktur (61).

Die allgemeine Form der Wortbildungsregel ist (96):

$$
\left.[\mathrm{x}] \rightarrow\left[\mathrm{K}_{\mathrm{K}}[\mathrm{x}]++^{+}\right] \rightarrow\left[\mathrm{K}_{\mathrm{TM}}[\mathrm{x}]+\ldots^{+}\right]++^{+}\right] \rightarrow
$$

In (96) steht $X$ für die morphonemische Repräsentation von $K$ oder R. Die lexikalische Kategorie wird jewells von $k$ bestimnt. $K$ indiziert deshalb stets die äuBerste klammer des Wortbildungsteils, während bereits von $K$ indizierte Klammern im Inneren - gemäB (DL30) die Funktion von TM übernehmen.

Soll anhand (96) das Nomen pracownik 'Arbeiter' gebildet werden, so wird das erste $\mathrm{K}$ durch $\mathrm{V}$ ersetzt, da der stamm der morphonemischen Repräsentation von pracowac 'arbeiten' entspricht, und folgender Merkmalkomplex hinzugefügt: 
(97)

$$
\left[\begin{array}{l}
+ \text { IMPERFEKTIV } \\
+ \text { INTRANSITIV } \\
- \text { PASSIV } \\
+ \text { TATIGKEIT } \\
+\mathrm{K}:\{+ \text { ova }+\}_{V}
\end{array}\right]
$$

Das zweite $K$ in (96) wird durch $N$ ersetzt und durch folgende Merkmale ergänzt:

(98)

$$
\left[\begin{array}{l}
+ \text { TRAGER } \\
+ \text { BELEBT } \\
+ \text { MENSCHLICH } \\
+ \text { BESTIMMUNG } \\
+K:\{+ \text { hik }+\}_{N}
\end{array}\right]
$$

Hinzu kommt das Genus-Merkmal:

$$
[+ \text { MASKULINUM ] }
$$

Soll die Wortbildungsregel den Forderungen (62) und (63) Rechnung tragen, so muß auch die morphonemische Repräsentation des Morphem-Markers $R$ berücksichtigt werden; also gilt für die Bildung des Nomen pracownik folgende Wortbildungsregel:

$$
\text { (100) } \mathrm{WT} \rightarrow\left[\begin{array}{r}
+ \text { IMPERFEKTIV } \\
+ \text { INTRANSITIV } \\
- \text { PASSIV } \\
+ \text { TÄTIGKEIT } \\
\left.V l_{R}-\operatorname{prac}+\right]+ \text { ova }+
\end{array}\right] \rightarrow
$$

Für die Einsetzung der $z u$ bildenden $W$-Basis in den entsprechenden terminalen Knoten der Satz-Basis gilt die zuordnung der in- 
hărenten und der kategorialen Merkmale zu der äuBersten K-Klammer, d.h., daB für die Einsetzung der W-Basis von pracownik die durch $\mathrm{N}$ gekennzeichnete äuBerste Klammer gilt. Für die w-Basis von pracownik gelten sowohl die Merkmale von $v$ als auch von $\mathrm{N}$; beide bilden einen Merkmalkomplex, der der morphonemischen Repräsentation von pracownik zugeordnet wird. Für die Ableitung der morphonemischen Endkette gelten die PL-Regeln und die DL-Regeln in $\S 7.1 .2$ und $\S 7.1 .3$.

Die Wortbildungsregel (100) lieBe sich - im Sinne von Lubaszewski (1982) - dadurch erweitern, daB vor der Bildung des V-Stammes eine Nominalisierung von $R$ vorgenommen würde. ${ }^{63}$ Dies bedeutete, daB mit dem Merkmal $N$ die $K$-Repräsentation $\{+\varnothing+\}_{N}$ nebst folgendem Merkmalkomplex der Wurzel zugeordnet werden müBten:

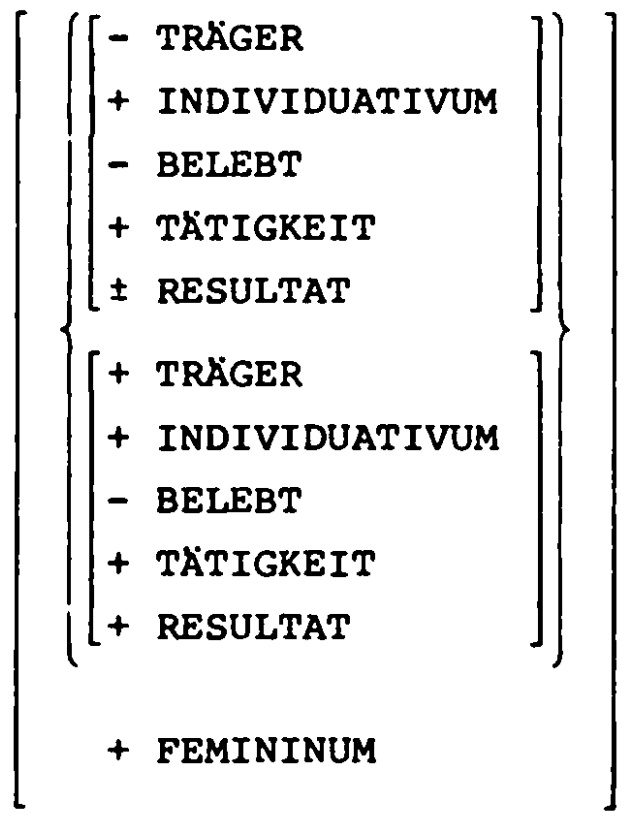

Der Merkmalkomplex (101) bezieht sich auf das Nomen praca, das zum einen die Bedeutung einer 'verrichteten Arbeit' im Sinne eines nicht gegenständichen Resultats oder aber im Sinne der 'Arbeit', an deren Verlauf - in Gegensatz zum Resultat - jemand teilnirumt oder teilhat, zum anderen die Bedeutung einer 'getanen Arbeit' im Sinne eines gegenständlichen Resultats.

In (100) gruppieren sich die Merkmale $z u$ einem in sich abgeschlossenen Merkmalkomplex, der der morphonemischen W-Basis von pracownik zugeordnet ist. Würde (101) mit aufgenommen, so mübte ein zusătzlicher Regelapparat aufgestellt werden, der die Unstimmigkeiten unter den Merkmalen beheben wirde. Abgesehen da- 
von ist das Merkmal [+ TÄTIGKEIT] in erster Linie mit dem verb verbunden, von dem auch sinnvollerweise ein mit 'Tätigkeit' assozilertes Nomen gebildet werden sollte; zumal dieses Nomen nicht die 'Tätigkeit' schlechthin, sondern deren Resultat bzw. deren Verlauf bezeichnet. Demnach muB zwischen der Nominalisierung in (100) und einer Nominalisierung (101) getrennt werden; es handelt sich zwar in beiden Fällen um eine Nominalisierung des Verbs, doch verlaufen beide Nominalisierungen anhand jeweils unterschiedlicher inhärenter Merkmale und führen zu entsprechend unterschiedlichen Ergebnissen. Daher gilt auch für die Bildung des Nomen praca eine getrennte Wortbildungsregel, die dieses $N$ nicht von $R$, sondern sinnvollerweise von $V$ bildet. Unter Nominalisierung wird hier allgemein die Bildung solcher Wörter verstanden, die der lexikalischen Kategorie $N$ angehören. Da die Nomina im Polnischen bei gleicher morphosyntaktischer Struktur unterschiedliche morphonemische Repräsentationen der Morphem-Marker aufweisen, wird im Hinblick auf die zu bildende W-Basis unterschieden zwischen:

(a) Derivationsregeln (DER-Regeln),

(b) Affigierungsregeln (AF-Regeln).

Die DER-Regel zeigt die morphemmarkierte und mit der jeweiligen lexikalischen Kategorie indizierte morphosyntaktische struktur der zu bildenden $W$-Basis einer Reihe von infragekommenden Nomina.

Die AF-Regel dagegen ordnet anhand des Merkmalkomplexes die entsprechende morphonemische Repräsentation der von der DER-Regel angezeigten morphosyntaktischen struktur $z u$ und bestimnt somit die w-Basis, d.1. die morphonemische struktur der betreffenden Nomina.

Voraussetzung für die AF-Regel ist also die DER-Regel ebenso, wie die Voraussetzung für die Einsetzung der morphonemischen Repräsentation die morphosyntaktische struktur ist.

Die Nomina im Polnischen werden von der Wurzel $R$, vom Nomen $N$, vom Adjektiv $A$ und vom Verb $V$ gebildet. Dementsprechend gelten folgende DER-Regeln, die für sämtliche AF-Regeln als Voraussetzung gelten: 
(102)

(DER 1)

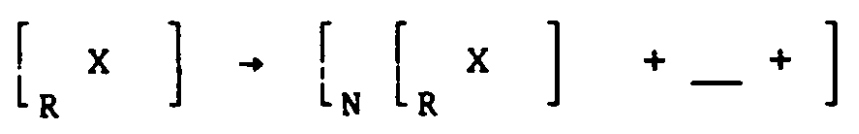

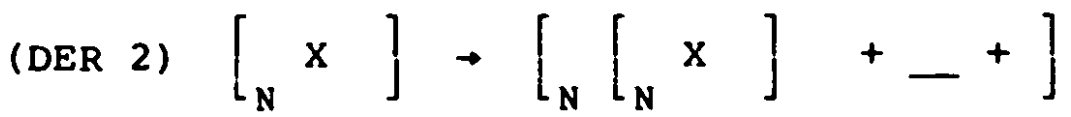

$(\operatorname{DER} 3)[\mathrm{X}] \rightarrow\left[{ }_{\mathrm{N}}[\mathrm{X}]+L^{+}\right]$

$(\operatorname{DER} 4)[v]+\left[_{N}[v x]+\ldots+\right]$

Die allgemeine Form der AF-Regel dagegen ist:

$$
\mathrm{K} \rightarrow\{+\ldots+\}_{\mathrm{N}} /\{+\ldots+\}_{\mathrm{TM}}
$$

Für die AF-Regel (103) gilt: K - in (96) - wird einer bestimmten morphonemischen Repräsentation mit dem Merkmal $N$ zugeordnet, wenn bestimmte Bedingungen erfüllt sind. $\mathrm{Zu}$ diesen Bedingungen zählen u.a. die morphonemische Repräsentation des TM sowie der Merkmalkomplex, durch die (103) ergänzt wird.

In (100) liegt eine DER-Regel vor, die zugleich die Funktion einer AF-Regel erfüllt. Da eine DER-Regel in (102) die Voraussetzung für eine groBe Anzahl von $A F-R e g e l n$ sein kann, wäre es nicht ökonomisch, die Nominalisierung im Polnischen mittels Regeln vom TYP (100) $z u$ beschreiben. Daher wird in der Numerierung der AF-Regeln auf die jeweilige vorausgesetzte DER-Regel hingewiesen, d.h., daB die AF-Regeln nach den DER-Regeln geordnet werden, indem z.B. alle AF-Regeln, die deverbale Nomina betreffen, durch (AF 4) - entsprechend (DER 4)-gekennzeichnet werden. 


\subsection{NOMINALISIERUNG IM POLNISCHEN}

Die Nominalisierung im Polnischen betrifft - den DER-Regeln in (102) entsprechend - die Bildung von "Wurzelnomina" und die Bildung von Stammomina. Die Bildung der Stammomina verlauft entsprechend (DER 2), (DER 3) und (DER 4). Die Bildung der "murzelnomina" besteht - (DER 1) entsprechend - darin, daB der morphonemischen Repräsentation von $R$ die morphonemische Repräsentation von $K$ zugeordnet wird, $d . h .$, das $K$ keinem bereits lexikalisch kategorisierten Wortstamn, sondern einer lexikalisch unmarkierten Wurzel zugeordnet wird. Die Wurzel stellt das einer Wortfamilie - vgl. $§ 6.2 .1 .1$ - Gemeinsame dar; sie ist als solches wiederkennbar und kann daher durch das Hinzufügen einer entsprechenden $\mathrm{K}$-Repräsentation mit einer entsprechenden Bedeutung assozilert werden.

Wollte man auf die Bildung der "Wurzelnomina" aus welchen auch immer Gründen in der Beschreibung der Nominalislerung des Polnischen verzichten, so mübte man eine ganze Reihe von Nomina ausschlieBen; so z.B. Kucharz 'Roch', ein Nomen, das aller Wahrscheinlichkeit nach - vgl. dazu SIA und SPV - aus dem Deutschen über das Tschechische ins Polnische aufgenommen worden ist und das der symchronischen Wortbildung schwierigkeiten bereitet. Dieses Nomen hat mit kuchnia 'Küche' die Wurzel $\{-$ kux +$\}$ gemeinsam, wobei die Bedeutung von kuch - nicht einmal die veraltete in DOR, nämlich 'Teig' - weder mit der von kucharz noch der von kuchnia etwas gemeinsam hat. Deshalb kann bel der Bildung des Nomen kucharz entweder davon ausgegangen werden, das zwischen kuch- und -arz keine Grenze anzunehmen ist, da der morphonemischen Repräsentation von kuch- keine lexikalische Kategorle zuzuordnen ist, oder es wird davon ausgegangen, dab der Sprecher des Polnischen [kux] als Wortsegment wiedererkennt, an das er entweder -arz oder $-n i-a$ hăngen kann, um das jeweilige Nomen zu bilden; dabei bleibt jedoch $\{-$ kux +$\}$ in der morphologischen Klassifikation die Wurzel $R$. Wollte man die erstgenannte Möglichkeit vorziehen, so dürfte auch zwischen kuch- und nia keine Grenze angenommen werden; diese Lösung widersprăche jedoch der Feststellung, daB gerade die Affigierung mit der $\mathrm{K}-\mathrm{Re}-$ präsentation $\{+\hat{K}+\}_{N} z u$ der Bildung einer von wenigen im Polnischen abgeschlossen nominalen Gruppen gehort. 
Das gleiche gilt für Nomina, wie z.B. syn 'Sohn' oder nos 'Nase', die durch die K-Repräsentation $\{+\emptyset+\}_{N}$ als Nomen klassifiziert werden; dies ebenfalls als Folge dessen, daB der Sprecher des Polnischen die Lautfolgen [sin] bzw. [nos] als R-Repräsentationen wiedererkennt und als Nomina klassifiziert, um sie u.a. in die Flexion einbeziehen zu können. Man könnte zwar Wörter wie kucharz, kuchnia, syn, nos als "Grund-" bzw. "Ausgangswörter" sehen, deren Bildung für die Beschreibung überflüssig ist, da sie Grundelemente des Lexikons für die Bildung von abgeleiteten Wörtern bzw. Derivaten darstellen; aber dann müBten einerseits die meisten Lehnwörter ausgeschlossen, anderseits müBte auf die nominale $K$-Repräsentation $\{+\emptyset+\}$ verzichtet werden, was $z u$ erheblichen Schwierigkeiten in der Beschreibung der Wortbildung führen würde; die wortbildung bestünde dann aus derivierten, also $\mathrm{zu}$ beschreibenden, und aus nichtderivierten, also nicht zu beschreibenden wörtern.

In der Beschreibung der Nominalisierung im Polnischen wird also davon ausgegangen, daß nicht nur stammomina, sondern auch die "Wurzelnomina" Gegegenstand sind, da die morphonemische wurzelrepräsentation als das einer wortfamilie Gemeinsame, wenngleich lexikalisch erst zu klassifizierende wiedererkennbar ist. Bei der Bildung der stammomina ist dagegen der Wortstamm bereits mit einer $K$-Repräsentation versehen, die durch das Hinzufügen einer weiteren K-Repräsentation - entsprechend (DL3O) - die Funktion eines Stamm-Markers TM erhält.

\subsection{WURZELNOMINA}

Die Wurzelnomina haben im Polnischen in der Regel die K-Repräsentation $\{+\emptyset+\}_{N}$. Dazu gehören die Bezeichnungen für LEBEWESEN, wie z.B. chzop, pan, wojt, brat, pies, kot, ptak 'Bauer/ /Mann, Herr, Vogt, Bruder, Hund, Katze, Vogel', kobieta, baba, kura, krowa, mysz 'Frau, Weib, Huhn, Kuh, Maus', zied, kon, ges 'Schwiegersohn, Pferd, Gans', die Bezeichnungen für KORPERTEILE, wie z.B. reka, noga, grabiet, pifsc, sierse, oko, ucho 'Hand, Bein/FuB, Rücken, Faust, Fell, Auge, Ohr', die Bezeichnungen für STOFFE, wie z.B. sok, miod, woda, piwo, wino 'saft, Honig, Wasser, Bier, Wein', die Bezeichnungen für WERKzEUGE, wie z.B. pzug, mzot, kosa, sito 'Pflug, Hammer, Sense, Sieb', 
die Bezeichnungen für BAUMTEILE, wie z.B. pien, korzen, gazqź, lisc, owoc, kora 'Stamm, Wurzel, Ast, Blatt, Frucht, Rinde' u. v.a.m.

Regel (AF 1.1) zeigt die Bildung dieser Nomina; auf die Notation der Genera wird verzichtet, weil die K-Repräsentation alle drei Genera impliziert.

$$
\begin{aligned}
& (A F 1.1) \quad K \quad \rightarrow \quad\{+\varnothing+\}_{N} /\left\{-{ }^{+}\right\}_{R} \\
& \left.\left[\begin{array}{c}
+ \text { TRAGER } \\
+ \text { BESTIMMTSEIN } \\
\left\{\left[\begin{array}{l}
+ \text { INDIVIDUATIVUM } \\
+ \text { BELEBT } \\
+ \text { INDIVIDUAT IVUM }
\end{array}\right]\right. \\
{\left[\begin{array}{l}
- \text { INELEBT } \\
- \text { BELEBT }
\end{array}\right]}
\end{array}\right]\right\}
\end{aligned}
$$

Der erste Merkmalkomplex in (AF 1.1) - innerhalb der Alternativklammer - betrifft die LEBEWESEN, der zweite betrifft die zählbaren, nicht belebten objekte, die dritte betrifft die STOFFE. ${ }^{64}$ Die Merkmale [+ TRÄGER; + BESTIMMTSEIN] - vgl. (90) und (95) - betreffen alle drei Merkmalkomplexe innerhalb der Alternativklammern. (AF 1.1) schlieBt das Merkmal [+ BESTIMMUNG] aus, d.h., daß mittels dieser Affigierungsregel nur Nomina gebildet werden können, die - entsprechend (95) - nur durch ein Festgelegtsein - mit abgeschlossenem Charakter - gekennzeichnet sind. während die durch Festlegung - mit offenem Charakter - gekennzeichneten Nomina andere Regeln und somit andere K-Repräsentationen voraussetzen.

Zu den Wurzelnomina müssen auch einige Nomina auf -arz und auf -erz gezählt werden, die zu den Nomina agentis gehören, also durch die Merkmale [+ TRAGER; + BESTIMMUNG] gekennzeichnet sind. Die unterschiedliche $K$-Repräsentation könnte entsprechend (PL $18(1))$ einheitlich durch $\left\{+\&_{2} \dot{r}+\right\}_{N}$ repräsentiert werden, wenn diese Lösung nicht allzu viel zusatzregeln erforderlich machen würde: deshalb wird die Affigierung durch zwei K-Repräsentationen, nämlich durch $\{+a \dot{r}+\}_{N}$ und durch $\{+e \dot{r}+\}_{N}$ bestimmt. zu 
diesen Nomina gehören:

\begin{tabular}{|c|c|c|}
\hline$(104)(1)$ & $\begin{array}{l}\text { kucharz } \\
\text { marynarz } \\
\text { grabarz } \\
\text { tragarz } \\
\text { walcarz }\end{array}$ & $\begin{array}{l}\text { 'Koch' } \\
\text { 'Matrose' } \\
\text { 'Totengräber' } \\
\text { 'Gepäckträger' } \\
\text { 'Walzwerkarbeiter' }\end{array}$ \\
\hline (ii) & $\begin{array}{l}\text { kusnierz } \\
\text { snycerz } \\
\text { salifierz } \\
\text { formierz } \\
\text { fazszerz } \\
\text { tancerz } \\
\text { solnierz }\end{array}$ & $\begin{array}{l}\text { 'Kürschner' } \\
\text { 'schnitzer' } \\
\text { 'Schleifer' } \\
\text { 'Eormer, GieBer' } \\
\text { 'Fälscher' } \\
\text { 'Tänzer', 6s } \\
\text { 'Soldat' }\end{array}$ \\
\hline
\end{tabular}

In (104) handelt es sich um eine Auswahl von Lehnwörtern aus dem Deutschen, die durch die morphonemischen Gegebenheiten des Polnischen zwei unterschiedliche $\mathrm{K}$-Repräsentationen haben. Die genuine $\mathrm{K}$-Repräsentation liegt-arz zugrunde, da diese bereits vor den Entlehnungen auf -arz im Polnischen vorhanden ist - so z.B. In gospodarz 'Herr, Hausherr'; vgl. dazu SiA - und da die genuinen Bildungen der Nomina agentis nicht auf -erz, sondern auf -arz auslauten; diachronisch könnte also behauptet werden, daB die worter wie grabarz - mhd. grabaere, greber morphonemisch stärker ins Polnische integriert worden sind als z.B. zoznierz - mhd. soldenaere, soldenier.

Bei einigen wörtern in (104) könnte der Eindruck entstehen, daß es sich um denominale Nomina handelt, so z.B. bei tancerz, da es im Polnischen auch das Nomen taniec 'Tanz' gibt. Die Kasus obliqui zeigen jedoch unverkennbar ein palatales $n$ in tanca / / taricu / taricem (GEN/DAT/INS). Also kann man hier nicht von einem nominalen Stamm ausgehen, sondern von der R-Repräsentation $\{- \text { tanc }+\}_{R}$; auch das Verb tarczyć 'tanzen' enthält ein palatales $x$. Bei kucharz könnte man diachronisch vom Nomen kuch 'Kuchen, Teig' - vgl. SkA - ausgehen, synchronisch jedoch nicht, da kuch im Gegenwartspolnisch - vgl DOR - ein technischer Ausdruck aus der Lebensmittelindustrie ist. Die Möglichkeit, nicht von $R$, sondern von $T M$ auszugehen, bieten formierz, fazszerz und zoznierz, nämlich von forma, fazsz, zozd 'Form, Falschung, sold'; doch zählen diese wörter insofern zur Minderheit, als deren nominaler Wortstamm ebenfalls aus dem Deutschen als eigenständiges Nomen entlehnt worden ist. 
Wie komnt es also dazu, daB im Polnischen Nomina wie die in (104) neben denominalen Zek-arz 'Arzt', garnc-arz 'Tobfer' gebildet werden können? Da die lexikalische Rategorie für die Kennzeichnung des Wortstames nicht in Frage-kount, kann die Bedingung für diese Affiglerungen auf der morphonemischen Ebene bestimmt werden, numlich anhand des R-Auslauts. Fur den Sprecher des Polnischen wird hier vorausgesetzt, daB er den konsonantischen R-Auslaut als Stammauslaut perzipiert, die entsprechende $\mathrm{K}$-Repräsentation hinzufügt und aufgrund dieser $\mathrm{K}$-Repräsentation das Nomen mit den Merkmalen eines Nomen agentis assozilert; denn man kann ja schlieblich nicht davon ausgehen, das der Sprecher des Polnischen die Wortbildung des Mittelhochdeutschen beherrscht.

Die zuordnung der -ers zugrunde liegenden $\mathrm{R}$-Repräsentation wird zum einen in Opposition zum haufigeren und uberwiegend in genuinen Nominalisierungen verwendeten -ars, zum anderen aber in Opposition zu den K-Repräsentationen in (105) und (106) gesehen. (105)

\begin{tabular}{|c|c|}
\hline $\begin{array}{l}\text { pionier } \\
\text { tapicer } \\
\text { secer } \\
\text { s sofer } \\
\text { akusser }\end{array}$ & $\begin{array}{l}\text { 'Pionier' } \\
\text { 'Tapezierer' } \\
\text { 'schriftsetzer' } \\
\text { 'Chauffeur' } \\
\text { 'Geburtshelfer' }\end{array}$ \\
\hline $\begin{array}{l}\text { 8stygar } \\
\text { husar }\end{array}$ & $\begin{array}{l}\text { 'steiger (in Bergewerk) } \\
\text { 'Husar' }\end{array}$ \\
\hline
\end{tabular}

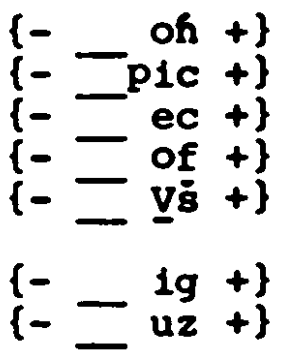

Die Frage, bei welchen wortern in (105) und (106) es sich un deutsche oder un franzosische Lehnworter handelt, ist fur die wortbildung nicht entscheidend. Entscheidend ist die Feststellung, das die jeweilige Reprasentation des $R$-Auslauts sowohl in (105) als auch in (106) die Repräsentationen des R-Auslauts in (104) ausschlieBen; aber (105) schliebt ebenfalls (106) aus. Das bedeutet, daB die zuordnung der entsprechenden $K$-Reprăsentationen von $-a r z,-e r z,-\beta r$ und $-a r$ bel gleichbleibender Bedeutung eines Nomen agentis bzw. elnes 'Trăgers' bezüglich 'Bestimmung' vom Auslaut der jewelligen R-Repräsentation, die in den melsten Făllen keinem genulnen Wortstamm entspricht, abhängig 1st: denn es gibt im Polnischen keine Nomina agentis, die z.B. auf *-oniers, *-picers,*-ecers, *-ofers, *-ussers oder auf *-ygars, *-uzarz auslauten, ebensowenig solche, die z.B. auf *-uchar, *-ynar, *-abar oder auf *-snier, *yoer auslauten. 
Also lassen sich folgende AF-Regeln aufstellen, die im Hinblick auf die -arz zugrunde liegende K-Repräsentation, die nicht nur in Wurzelnomina, sondern auch in den regulären Stammnomina - und dort uberwiegend - verwendet wird, in der hier angegebenen Reihenfolge angewandt werden sollten; zunächst für (105): $(A F 1.2 .1)$

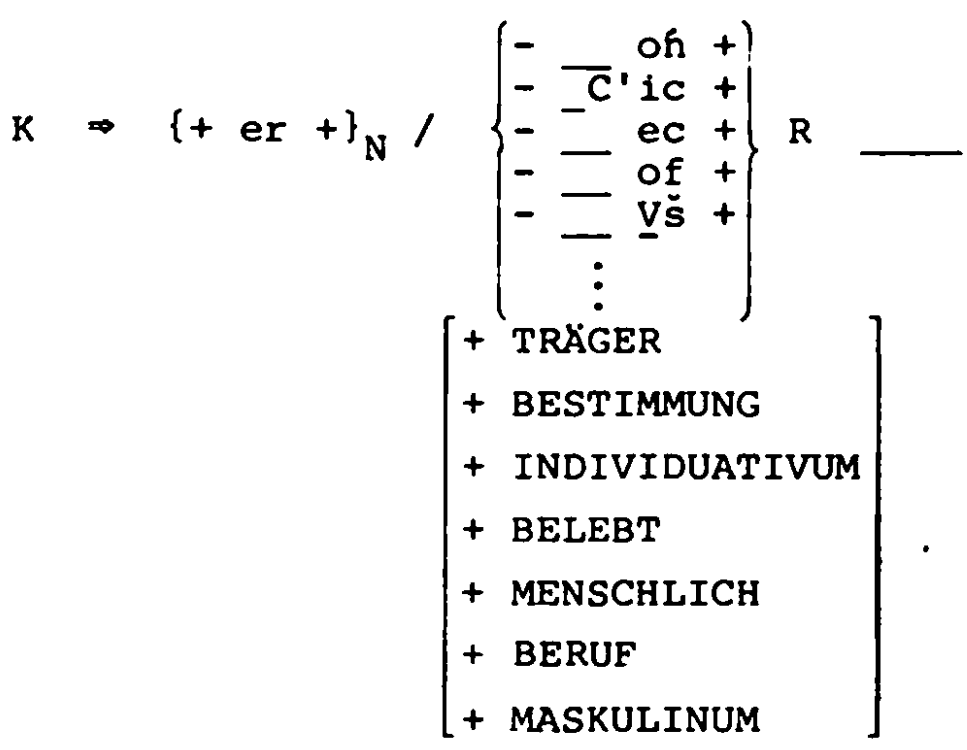

Für (106) gilt folgende AF-Regel:

$$
\begin{aligned}
& \text { (AF 1.2.2) } \\
& K \rightarrow\{+\operatorname{ar}+\}_{N} /\left\{\begin{array}{c}
--i g+ \\
- \\
\vdots
\end{array}\right\} R \\
& \text { [WIE IN (AF 1.2.1)] }
\end{aligned}
$$

Für (104(ii)) gilt folgende AF-Regel:

(AF 1.2.3)

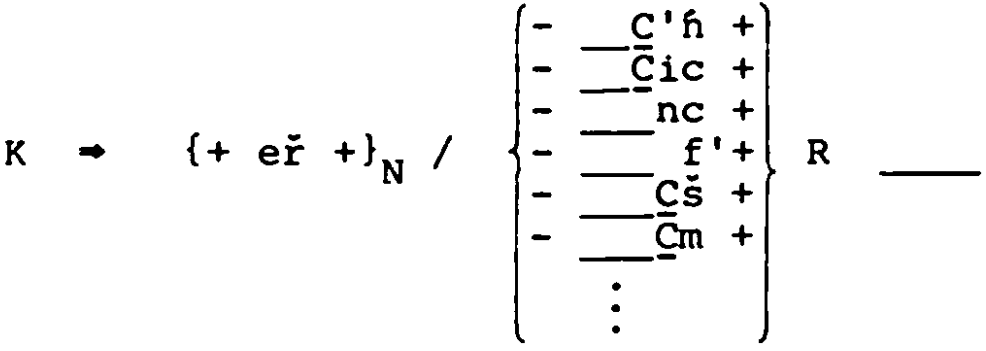

$$
\begin{aligned}
& \text { [WIE IN (AF 1.2.1)] }
\end{aligned}
$$

Und für (104(i)) gilt schlieBlich folgende AF-Regel:

$$
\begin{aligned}
(A F 1.2 .4) \mathrm{K} \rightarrow\{+a \check{r}+\}_{N} /\left\{-\frac{\text { SONST }}{R}^{+\}} \mathrm{R}-\right. \\
\\
{[\text { WIE IN }(A F 1.2 .1)] }
\end{aligned}
$$




\subsection{DENOMINALE NOMINA}

Die denominalen Nomina werden gemäß Regel (DER 2) gebildet. Die von ihnen bezeichneten objekte reichen $u . a$. von Personen, die nach Beruf, Beschäftigung, Hobby, Amt u.ä. bezeichnet werden, über Gegenstände, die für bestimmte Dinge bestimmt und nach denen benannt sind, bis zu Räumlichkeiten, die für Träger bestimmter Funktionen bestimnt sind. Den meisten dieser denominalen Nomina sind die Merkmale (107) gemeinsam:

(107)

$$
\left[\begin{array}{l}
+ \text { FUNKTION } \\
+ \text { TRAGER } \\
+ \text { INDIVIDUATIVUM }
\end{array}\right]
$$

Diese Merkmale sind in (90), (92), (94) und (95) definiert und werden in den aufzustellenden AF-Regeln, in denen sie nicht notiert sind, als Bedingungen impliziert.

Die Regeln (AF 1.2.1 - AF 1.2.4) sind u.a. aufgrund der Feststellung aufgestellt worden, daß es im Polnischen eine Reihevon Berufsbezeichnungen gibt, die nicht vom stamm, sondern von der Wurzel gebildet werden, $d . h$., daB die w-Basis dieser wörter aus der jeweiligen morphonemischen Repräsentation der wurzel $R$ und des nominalen $\mathrm{K}$ besteht. Im Gegensatz dazu gibt es eine ganze Reihe von Bezeichnungen für Personen bezüglich ihres Berufs, ihrer Beschäftigung, ihres Amts, die von einem nominalen Stamm gebildet werden, indem die nominale $K$-Repräsentation $\{+(*,) a \check{r}+\}_{N}$ hinzugefügt wird. Dabel enthalten die einen unter ihnen den Konsonantenwechsel des nominalen TM-Auslauts, symbolisiert durch $(*$,$) im Sinne der Regeln (PL8 - PL15), die anderen - wie be-$ reits in (10) angedeutet - enthalten keinen Konsonantenwechsel im Stammauslaut. Dazu einige Beispiele in (108):

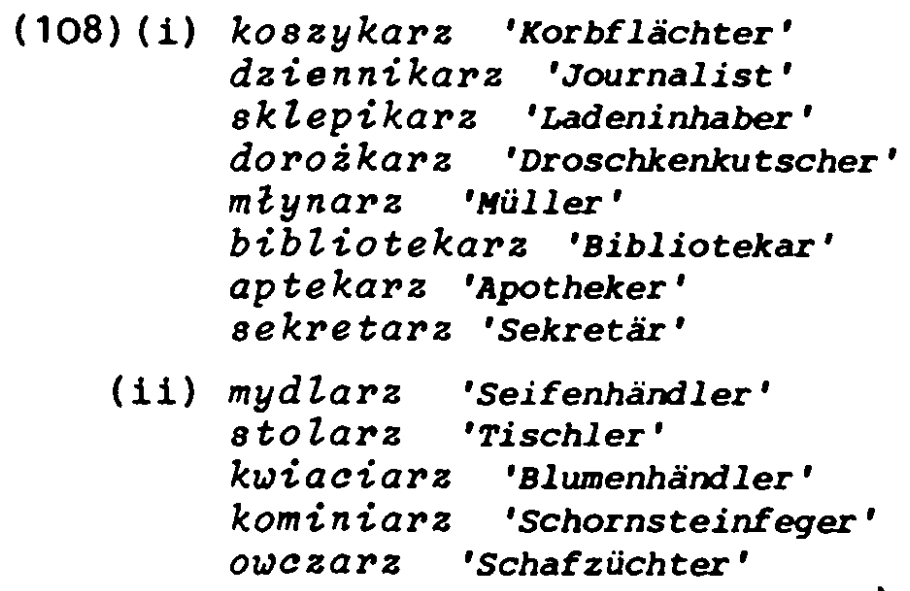

- koszyk 'Korb'

- dziennik 'Journal'

- sklepik 'Laden'

- doroika 'Droschke'

- mzyn 'mühle'

- biblioteka'Bibliothek'

- apteka 'Apotheke'

- sekret 'Geheimnis'

- mydzo 'Seife'

- stoz 'Tisch'

- kwiat 'Blume'

- komin 'schornstein'

- ouca 'schaf' 


\begin{tabular}{|c|c|c|c|c|}
\hline & $\begin{array}{l}\text { mlecsars } \\
\text { knajpiars }\end{array}$ & $\begin{array}{l}\text { 'Milchiann' } \\
\text { 'Tneipemirt' }\end{array}$ & $\begin{array}{l}\text { - mleko } \\
\text { - knajpa }\end{array}$ & $\begin{array}{l}\text { 'Milch' } \\
\text { 'Kneipe' }\end{array}$ \\
\hline (11) & $\begin{array}{l}\text { knajpiarz } \\
\text { kobieciarz } \\
\text { gawpdziarz } \\
\text { karciars }\end{array}$ & $\begin{array}{l}\text { 'Zechbruder' } \\
\text { 'Schürzenjäger' } \\
\text { 'Plauderer' } \\
\text { 'Kartenspieler' }\end{array}$ & $\begin{array}{l}\text { - knajpa } \\
\text { - kobieta } \\
\text { - gaweda } \\
\text { - karta }\end{array}$ & $\begin{array}{l}\text { 'Kneipe' } \\
\text { 'Prau' } \\
\text { 'Plauderei' } \\
\text { 'Karte' }\end{array}$ \\
\hline
\end{tabular}

Der durch $(*$,$) symbolisierte Konsonantenwechsel betrifft die$ worter $(108(1 i))$ und $(108(11 i))$. Die Nomina dieser beiden Reihen werden durchweg von Nomina gebildet, die die K-Repräsentation $\{+\emptyset+\}_{N}$ haben; dies im Gegensatz - mit Ausnahne von bibliotekarz, aptekarz, sekretarz - $2 \mathrm{u}(108(1))$. Die nominalen TM-Repräsentationen in $(108(1))$ sind:

(109)

$$
\begin{array}{ll}
\{+/, i k+\}_{\mathrm{EV} / \mathrm{N}} & k 08 z-y k, 8 k l e p-i k \text { (Diminutiva) } \\
\{+8 \mathrm{k}+\}_{\mathrm{N}} / \mathrm{EV} & \text { doroz-ek (GBN PL) } \\
\{+ \text { in }+\}_{\mathrm{N}} & m l-e c-m z-y n \text { 'mahlen - mühle }
\end{array}
$$

Als zusatzliches Beispiel für die Notwendigkeit der Unterscheidung zwischen (108(1))-aufgrund (109) sowle (108(11)) und (108 (111)) - sei genannt:

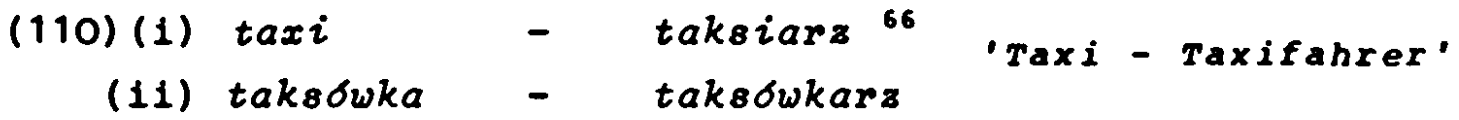

Da beide von $N$ gebildeten Nomina in (110) ein und dieselbe Bedeutung haben, kann man feststellen, das der Konsonantenwechsel in (110(1)) nicht durch die dem Nomen inhärenten Merkmale, sondern morphonemisch bedingt ist; dies gilt auch für die Beispiele in $(108(11))$ und $(108(111))$. Um der Unterscheidung $2 w i s c h e n$ $(110(1))$ und $(110(1 i))$ Rechnung $z u$ tragen, werden $z w e 1$ AF-Regeln aufgestellt, nămlich: 1) gelten die TM-Repräsentationen (109), so wird die K-Repräsentation $\{+a \dot{r}+\}_{N}$ zugeordnet; 2) sonst wird die $K$-Reprasentation $\{+* \text {, ar }+\}_{N} z$ ugeordnet. Die erste AF-Regel betrifft also (108(i)), die zweite dagegen (108 (11)) und (108(111)): siehe: (AF 2.1.1) und (AF 2.1.2).

Die Feststellung, daB die unterschiedliche K-Repräsentation morphonemisch bedingt ist, schliest nicht aus, das für diese Nomina auch die inhärenten Merkmale bestimnt werden müssen, da diese ja mit der R-Repräsentation assozilert werden. Im Falle von knajpiara gilt einmal in (108(1i)) die Bedeutung 'Beruf' und einmal in (108(111)) die Bedeutuna 'Beschäftiqung, Liebhaberei'. Morphosyntaktisch liegt also zwischen diesen beiden Bedeutungen 
kein Unterschied vor; der Bedeutungsunterschied ist allein im semantischen Bereich zu finden, nämlich darin, das hier im normalen Sprachgebrauch zwischen Beruf und Beschätigung unterschieden wird. Die unterschiedliche Bedeutung kann durch folgende Paraphrasen verdeutlicht werden:

$$
\begin{gathered}
\text { (111)(1) ten, co ma knajpe } \\
\text { posiada } \\
\text { prowadzi } \\
\text { 'einer, der eine Kneipe hat/ besitzt/ führt } \\
\text { (11) ten, co vysiaduje w knajpie } \\
\text { 'einer, der in der Kneipe herumzusitzen pflegt. }
\end{gathered}
$$

Der Bedeutungsunterschied kann auf der Ausdrucksebene durch die deverbalen Nomina posiadacz 'Besitzer' und wysiadywacz 'Herumsitzer' wiedergegeben werden; doch weist keines der beiden worter auf das objekt des 'Besitzens' bzw. auf den Ort des 'Herumsitzens' hin, daher mus knajpiarz beide Bedeutungen in sich vereinen. Den Uberlegungen in $\S 5.4 .1$ zur Folge muB also die $k-R e-$ präsentation $\{+(*,) \text { ar }+\}_{N}$ mit einer 'Tätigkeit' assoziiert werden, deren unmarkiertes objekt den nominalen Wortstamm repräsentiert; also handelt es sich sowohl im Falle des 'Kneipenwirts' als auch im Falle des 'Zechbruders' um 'Trăger' einer bestimmten 'Tatigkeit', die auf ein bestimmtes objekt gerichtet ist, welches den Wortstamm repräsentiert: das gleiche gilt für die anderen Beispiele in (108).

Bei allen diesen Beispielen handelt es sich also un denominale Nomina, die sowohl 'Beruf' als auch 'Liebhaberei' bezeichnen können, dies bei gleicher morphonemischer Repräsentation. Vergleicht man diese worter 2.B. mit dem deverbalen Nomen pijak 'Säufer', für den folgende Paraphrase gilt:

$$
\begin{aligned}
& \text { ten, co pije /nazogowo wbdke,... / } \\
& \text { 'einer, der (gewohnheitsmäBig Schnaps) trinkt }
\end{aligned}
$$

so kann man feststellen, daB es der Verbalstamn ist, der eine andere K-Repräsentation zur Folge hat. Aber auch für dieses Nomen gelten die Merkmale u.a. der 'Tätigkeit', die allerdings durch den Verbalstamm ausgedrückt wird. Infolge dessen kann die k-Repräsentation auch mit dem Adverb nalogowo assozilert werden, das die Tätigkeit näher bestimnt. Allerdings kann bei diesen Beispiel weder von 'Beruf' noch von 'Liebhaberei' die Rede sein, allerdings von einer Art 'Beschaftigung'. 
Für die AF-Regeln können demnach die implizierten inhărenten Merkmale (107) durch [+ BESTIMMUNG] und [+ TATIGKEIT] im sinne der Definitionen (95) ergänzt werden, wobei das Merkmal [+ BERUF] für die 'berufliche Ausübung' dieser 'Tatigkeit' und das Merknal [- BERUF] für alle anderen Arten der 'Ausübung' dieser 'Tatigkeit' stehen; denn neben stolarz 'Tischler', wo eine ausgesprochen 'berufliche Tätigkeit' bezelchnet wird, ${ }^{67}$ werden entsprechend (108) auch solche 'Tätigkeitsträger' bezeichnet, wie 2.B. pizkarz 'FuBballspleler', bramkarz 'Tormann', narciarz 'Skilăufer', Zyziwiarz 'Schlittschuhlăufer', worter also, in deren Bedeutung die Grenzen zwischen 'Beruf' und 'Nicht-Beruf ' fliebend sind; im Falle des Nomen rybiarz '(begeisterter) Fischesser' steht sogar die K-Repräsentation in Opposition $2 u$ der in rybak 'Fischer'; doch handelt es sich hier um eine von wenigen Ausnahmen.

Die AF-Regel für die worter (108) wird dem durchzuführenden bzw. nicht durchzufuhrenden Konsonantenwechsel entsprechend getrennt aufgestellt. Als Bedingungen für den nicht durchzuführenden Konsonantenwechsel gelten die TM-Reprăsentationen (109) - diese auch für die Nomina piz-k-arz und bram-k-arz - sowie die stamm- Repräsentationen der Nomina bibliotekarz, aptekarz, sekretarz samt TM-Repräsentation $\{+\emptyset+\}_{N} \cdot 68$

Für $(108(1))$ gilt folgende AF-Regel:

(AF 2.1 .1$)$

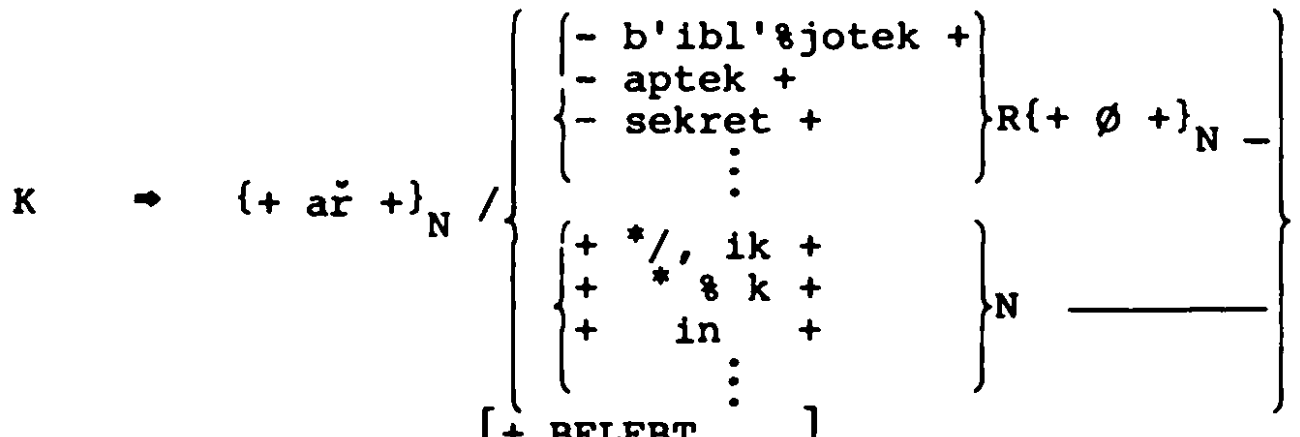

$$
\begin{aligned}
& {\left[\begin{array}{l}
+ \text { BELEBT } \\
+ \text { MENSCHLICH } \\
+ \text { BESTIMMUNG } \\
+ \text { TATIGKEIT } \\
\pm \text { BERUF } \\
+ \text { MASKULINUM }
\end{array}\right]}
\end{aligned}
$$

Fur all die Nomina in (108), in denen der Konsonantenwechsel bel der Hinzufügung der $K$-Repräsentation durchgeführt wird, gilt 
folgende AF-Regel:

$(A F 2.1 .2) \quad K \quad \rightarrow \quad\{+*, a \ddot{x}+\}_{N} /\{+\emptyset+\}_{N}$

$$
\text { [ WIE (AF 2.1.1)] }
$$

Aufgrund des Festgestellten kann uber die Bildung der Nomina in (108) folgendes zusammengefaßt werden:

(108.1)(a) Die Nomina (108) sind ausschlieblich denominal;

(b) Der Konsonantenwechsel in Stammauslaut gehört dann zur allgemeinen Tendenz, wenn der stamm nicht durch die TM-Repräsentationen (109) sowie durch bestimmte morphonemische Auslautreprăsentationen bestimmt ist;

(c) Die Nomina (108) sind Maskulina;

(d) Die Nomina (108) bezeichnen 'Tätigkeitsträger' im Sinne von 'Beruf' und 'Nicht-Beruf'.

Die Maskulina (108) sind gegenüber den Feminina (113) sowohl bezüglich des Genus als auch bezüglich der K-Repräsentation, die bei den Feminina hinzugefügt wird, unmarkiert; vgl. $\S 2.1$. Das gleiche gilt für die Maskulina (104), (105) und (106). Die Feminina (113) unterscheiden sich von den Maskulina einzig und allein durch das Genus und die K-Repräsentation.

$\begin{array}{ll}\text { (113) (i) dziennikarka 'Journalistin' } & \text { - dziennikarz } \\ \text { sklepikarka 'Ladeninhaberin' } & \text { - sklepikarz } \\ \text { mZynarka 'Müllerin' } & \text { - mynarz } \\ \text { bibliotekarka 'Bibliothekarin' } & \text { - bibliotekarz } \\ \text { aptekarka 'Apothekerin' } & \text { - aptekarz } \\ \text { sekretarka 'Sekretärin' } & \text { - sekretarz } \\ \text { (ii) mydlarka 'Seifenherstellerin' } & \text { - mydlarz } \\ \text { kwiaciarka 'Blumenhändlerin' } & \text { - kwiaciarz } \\ \text { mleczarka 'Milchhändlerin' } & \text { - mleczarz } \\ \text { (iii) karciarka 'Kartenspielerin' } & \text { - karciarz } \\ \text { Zyzwiarka 'Schlittschuhläuferin' - Zyziarz } \\ \text { koszykarka 'Basketballspielerin' - koszykarz }\end{array}$

Hat ein maskulines Nomen, das entsprechend ( $A F$ 2.1.1) und ( $A F$ 2.1.2) gebildet wird, kein 'belebtes' feminines Pendant, d.h. sind Frauen in diesen Berufen bzw. Nicht-Berufen nicht vertreten, so dient das feminine 'nicht belebte' Pendant des maskulinen Nomen entweder zur Bezeichnung all dessen, was zum Aufgabenbzw. Interessenbereich des MASKULINUM gehört, so z.B. zu der 'Gesamtheit der Tischlerarbeiten in einem Haus': stolarka, oder zur Bezeichnung des 'Materials', aus dem das Produkt herge- 
stellt wird, z.B. koszykarka 'Weidenrute', oder aber zur Bezeichnung von 'Kleidungsstücken', z.B. marynarka 'Jacke', kominiarka '(eine bestimnte) Mutze'. In Falle von koszykarka liegen - vgl. (113(1ii))-wiederum zwei Bedeutungen vor, die sich u.a. durch [ \pm BELEBT] von einander unterscheiden. Daher wird davon ausgegangen, daB ein und derselbe Ausdruck von zwei getrennt aufzustellenden AF-Regeln gebildet wird, dies jeweils entsprechend seiner Bedeutung.

Voraussetzung für die Bildung der Feminina (113) sowie der hier genannten Feminina mit dem Merkmal [- BELEBT] ist die Bildung der Maskulina entsprechend (AF 1.2.1 - AF 1.2.4), (AF 2.1.1) sowie (AF 2.1.2). In den aufzustellenden AF-Regeln werden statt der vorausgesetzten AF-Regeln die maskuline TM-Repräsentation sowie die Merkmale [+ MENSCHLICH; + MASKULINUM] notiert. Fur (113) gilt also folgende AF-Regel:

(AF 2.2.1)

$$
\left[\begin{array}{l}
+ \text { MENSCHLICH } \\
+ \text { MASKULINUM }
\end{array}\right]
$$

$$
\begin{aligned}
K \rightarrow\{+* k+\}_{N} /\{+(*,) \text { ar }+\}_{N} \\
\\
{\left[\begin{array}{l}
+ \text { BELEBT } \\
+ \text { MENSCHLICH } \\
+ \text { BESTIMAUNG } \\
+ \text { TATIGKEIT } \\
\pm \text { BERUF } \\
+ \text { FEMININUM }
\end{array}\right] }
\end{aligned}
$$

Die femininen Pendants mit dem Merkmal [- BELEBT], wie die genannten koszykarka, marynarka, kominiarka, stolarka bezeichnen keine 'Tătigkeit', sondern entweder 'zugehörigkeit', wie z.B.. stolarka, als 'Gesamtheit der Arbeiten', die zum Tischler gehören, oder 'Instrumentalitat', wie z.B. koszykarka, als 'Weidenrute' zur Herstellung von Korben. Entsprechend (95) gilt deshalb auch nicht [+ BESTIMMUNG], sondern [+ BESTIMMTSEIN]. Das 'Bestimmtsein' im sinne von 'Zugehorrigkeit' oder 'Instrumentalität' wird in den Paraphrasen deutlich, die diesen Feminina zugrundegelegt werden können, wie z.B. wiklina dia koszykarza 'Rute für den Korbflächter' oder praca dla stolarsa 'Arbeit für den Tischler' oder aber maszyna / obrabiarka dla tokarza - tokarka "Maschine" für den Dreher - Drehbank'. Für diese Nomina 
gilt folgende AF-Regel:

(AF 2.2.2)

$$
\begin{aligned}
& {\left[\begin{array}{l}
+ \text { MENSCHLICH } \\
+ \text { MASKULINUM }
\end{array}\right]} \\
& \mathrm{K} \quad \rightarrow \quad\{+* \mathrm{k}+\}_{\mathrm{N}} /\{+(*,) a \dot{\mathrm{r}}+\}_{N} \\
& \text { - BELEBT } \\
& \text { - RAUMLICH } \\
& + \text { BESTIMMTSEIN } \\
& \left\{\begin{array}{l}
{[+ \text { ZUGEHORIGKEIT }]} \\
{[+ \text { INSTRUMENTALITAT }]} \\
+ \text { FEMININUM }
\end{array}\right\}
\end{aligned}
$$

Das Merkmal [- RÄUMLICH] wird in (AF 2.2.2) als Abgrenzung gegenüber solchen demaskulinen Nomina, wie z.B. stolarnia 'Tischlerei' - vgl. (114) - gesetzt. Das Merkmal [+ INSTRUMENTALITAT] wird nicht weiter ersetzt, weil hier sowohl [+ MITTEL] als auch [ + MASCHINE] zutreffen.

Zusammenfassend läBt sich zu der Bildung der Feminina (113) und der 'Nicht-Belebten' unter ihnen folgendes feststellen:

(113.1)(a) Die Feminina (113) sind von des Maskulina (108) gebildet und tragen - außer dem Genus - die gleichen Merkmale wie die Maskulina sofern sie 'belebt' sind:

(b) Voraussetzung für die Bildung der Feminina (113) ist die Bildung der Maskulina (108);

(c) Mit dem Merkmal [- BELEBT] sind die Merkmale des 'Bestimmtseins' im Sinne von 'Zugehörigkeit' oder 'Instrumentalität' verbunden;

(d) Sowohl die 'belebten' als auch die 'nicht belebten' Feminina, die entsprechend ( $A F$ 2.2.1) sowie (AF2.2.2) gebildeł werden, sind gegenüber den Maskulina, von denen sie gebildet werden, markiert; dies bezüglich Genus und morphonemischer $K$-Repräsentation;

(e) Für die phonologische Endableitung gilt u.a. (PL26).

Wie bereits mit dem Beispiel stolarnia angedeutet, dienen die Maskulina (108) ebenfalls zur Bildung einer stattlichen Anzahl von Feminina, die bezüglich [+ RAUMLICH] gegenüber den 'nicht belebten Feminina vom Typ stolarka und den Maskulina (108) be- 
züglich Genus markiert sind. Dazu gehören u.v.a.:

$(114)(1)$

kwiaciarnia 'Blumenladen'
mleczarnia 'Milchladen'
owczarnia 'Schafstall'
stolarnia 'Tischlerei'

(11) karciarnia 'Kartenspielort' narciarnia 'Skiablageraum' kawiarnia 'Café'
- kwiaciara

- mieczarz

- owczarz

- stolarz

- karciarz

- narciarz

- kawiarz

Die Nomina in (114) haben u.a. die Merkmale [- BELEBT; + RAUMLICH] gemeinsam. Die Reihen (114(i)) und (114(11)) entsprechen den Reihen $(108(11))$ und $(108(1 i 1))$; das bedeutet jedoch nicht, daB (108(1)) kelne răumlichen Entsprechungen haben kann; so wird z.B. koszykarnia 'Korbflechtere1' von koszykarz 'Korbflechter' gebildet. Doch im Falle solcher worter wie z.B. aptekarz, bibliotekarz, die bereits nach dem 'Raum' Ihrer 'Tatigkeit' benannt sind, ist die Bildung eines Femininum vom TYP (114) nicht mehr möglich. ${ }^{69}$ Ausgeschlossen werden hier auch die Maskulina (104), (105) und (106), die offensichtlich als Lehnwörter in die Wortbildung nicht ausreichend genug integriert worden sind, um sie hier aufnehmen zu können. Für (114) gllt folgende AF-Regel:

(AF 2.3.1)

$$
\left[\begin{array}{l}
+ \text { MENSCHLICH } \\
+ \text { MASKULINUM }
\end{array}\right]
$$

$$
\begin{aligned}
& K \quad \Rightarrow\{+*, 8 \hat{\mathrm{N}}+\}_{\mathrm{N}} /\{+(*,) a \dot{\mathrm{r}}+\}_{\mathrm{N}} \\
& {\left[\begin{array}{l}
- \text { BELEBT } \\
+ \text { RAUMLICH } \\
+ \text { BESTIMMTSEIN } \\
+ \text { ZUGEHORIGKEIT } \\
+ \text { FEMININUM }
\end{array}\right]}
\end{aligned}
$$

Das Merknal [+ RAUUMICH] kann durch Paraphrasen vom Typ: pracownia dia stolarza 'Werkstatt für den Tischler' oder pracownia dla koszykarza 'Werkstatt für den Korbflechter' verdeutlicht werden: gleichzeitig verdeutlicht die $P$ d a 'für' die Merkmale [+ BESTIMMTSEIN; + ZUGEHORIGKEIT].

Die Annahme der Vokal-Null-Alternation in der K-Repräsentation geschieht im Hinblick auf die mögliche Diminutivierung, so z.B. In kawiar-en-k-a '(kleines) Cafe', wobel die Entpalatalisierung (PL26) entspricht. 
Zur Bildung der Feminina (114) läBt sich zusammenfassend feststellen:

(114.1)(a) Die Feminina (114) werden von den Maskulina (108) gebildet; daher ist die Bildung der Maskulina (108) Voraussetzung für die Bildung der Feminina (114);

(b) Gegenüber den Feminina (113) sind die Feminina (114) bezüglich [+ RAUMLICH] markiert;

(c) Gegenüber den Maskulina (108) sind die Feminina (114) bezüglich Genus und K-Repräsentation $\{+*, 8 \mathrm{~h}+\}_{\mathrm{N}}$ markiert:

(d) Die Feminina (114) sind durch die Merkmale des 'Bestimmtseins' und der 'Zugehorrigkeit' gekennzeichnet;

(e) Für die phonologische Endableitung gilt u.a. (PL26).

Die K-Repräsentation in (AF 2.3.1) gilt auch dann für die Bildung von Raumbezeichnungen, wenn diese nicht von (108) gebildet werden, sondern vom 'Material', das darin verarbeitet, oder vom 'Produkt', das darin hergestellt wird. zu diesen Nomina gehören $\mathbf{u} \cdot \mathbf{v} \cdot \mathbf{a} \cdot \mathbf{:}$

$\begin{array}{lll}\text { papiernia } & \text { 'Papierfabrik' } & \text { - papier 'Papier' } \\ \text { cegielnia 'Ziegelei' } & \text { - cegza 'Ziegel' } \\ \text { lakiernia } & \text { 'Lackiererei/Lackfabrik'- Lakier 'Lack' } \\ \text { cukiernia 'Zuckerbäckerei' } & \text { - cukier 'Zucker' }\end{array}$

Anhand der Paraphrasen, wie z.B. fabryka, co produkuje cegzy 'Fabrik, die ziegeln herstellt' - vgl. auch (47.4.3) - oder fabryka, co produkuje lakier 'Fabrik, die Lack herstellt' läBt sich feststellen, daB es sich um das inhärente Merkmal des 'Bestimmtseins' im Hinblick auf 'Instrumentalität' handelt; denn entsprechend (95) sind die 'Räume' bezüglich ihrer 'Funktion', die in der Herstellung des vom unmarkierten Wortstamm ausgedrückten 'produkts' festgelegt. Demnach sind die Nomina (115) gegenüber den Nomina (114) bezüglich [+ INSTRUMENTALITÁT] markiert, während (114) gegenüber (115) bezüglich [+ zUGEHORIGKEIT] markiert sind. Gemeinsam haben sie u.a. das Merkmal [+ BESTIMMTSEIN] SOWIe [+ RÄUMLICH] .

Die Nomina (115) stehen in Opposition zu den Nomina (116) sowohl bezüglich der $\mathrm{K}$-Repräsentation als auch bezüglich des den Wortstamm kennzeichnenden Merkmals [ \pm BELEBT]. In (115) ist nämlich die 'Nicht-Belebtheit' des Wortstammes Voraussetzung 
für die zuordnung der $K$-Repräsentation $\{+*, 8\}+\}_{N}$, wăhrend in (116) das Merkmal [+ BELEBT] die zuordnung einer anderen K-Repräsentation zur Folge hat.

Fủr (115) gilt folgende AF-Regel:

(AF 2.3.2)

[ - BELEBT ]

$\begin{aligned} & K=\{+*, 8 \hat{f}+\}_{N} /\{+\emptyset+\}_{N} \\ & {\left[\begin{array}{l}- \text { BELEBT } \\ + \text { RAUMLICH } \\ + \text { BESTIMMTSEIN } \\ + \text { INSTRUMENTALITAT } \\ + \text { FEMININUM }\end{array}\right] }\end{aligned}$

Im Gegensatz zu den Raumbezeichnungen (115) werden die Raumbezeichnungen (116) von Nomina gebildet, die durch die Merkmale [ + BELEBT; - MENSCHLICH] gekennzeichnet sind. Dazu geh3̈ren:

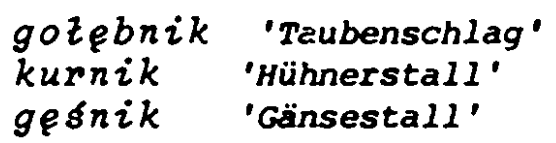

- gozqb 'Taube'
- kura 'Huhn'
- ge\& 'Gans'

Die Paraphrasen lauten z.B. budynek dla gozebi 'Gebăude für Tauben' oder budynek dla gesi 'Gebäude für Gänse'. Wle für (114) gelten also auch für (116) die Merkmale des 'Bestimmtseins' im Sinne eines 'Festgelegtseins' bezüglich einer 'Zugehörigkeit' gegenüber dem vom unmarkierten Nominalstamm bezeichneten objekt, das in (116) ein 'Tier' ist.

Für (116) gilt folgende AF-Regel:

(AF 2.4.1)

$$
\left[\begin{array}{l}
+ \text { BELEBT } \\
- \text { MENSCHLICH }
\end{array}\right]
$$

$$
\begin{aligned}
& K \rightarrow\{+*, \text { hik }+\}_{N} /\{+\varnothing+\}_{N} \\
& {\left[\begin{array}{l}
- \text { BELEBT } \\
+ \text { RAUMLICH } \\
+ \text { BESTIMMTSEIN } \\
+ \text { ZUGEHORIGKEIT } \\
+ \text { MASKULINUM }
\end{array}\right] }
\end{aligned}
$$

Zusammenfassend läBt sich zur Bildung der Nomina (115) und der Nomina (116) folgendes feststellen:

(115.1)(a) Die Feminina (115) werden von Nomina gebildet, die im Gegensatz zu (114) durch [- BELEBT] gekennzeich- 
net sind und die das 'Produkt' bzw. das 'Material' bezeichnen, die in dem 'Raum', den das $z$ u bildende Nomen bezeichnet, hergestellt bzw. verwendet werden:

(b) Gegenüber (114) sind die Nomina (115) bezüglich des Merkmals [+ INSTRUMENTALITÁT] markiert;

(c) Für die phonologische Endableitung gilt u.a. (PL26).

(116.1) (a) Die Maskulina (116) werden wie die Feminina (114) von 'belebten' Nomina gebildet, doch im Gegensatz zu (114) handelt es sich in (116) um 'Tiere';

(b) Die Nomina (116) haben mit (114) und (115) das Merkmal [+ RÄUMLICH] gemeinsam;

(c) Gegenüber (115) sind die Nomina (116) - ebenso wie (114) - bezüglich [+ zUGEHORIGKEIT] markiert;

(d) Für die phonologische Endableitung gilt u.a. (PL26).

Neben den Maskulina (116) gibt es eine ganze Reihe von Maskulina, die die gleiche K-Repräsentation haben, doch von 'nicht belebten' Nomina - darunter auch von deverbalen - gebildet werden. Dazu gehören u.v.a.:

$\begin{array}{ll}\text { (117) (i) pibrnik 'Federkasten' } & \text { - pioro '(Stahl-)Feder' } \\ \text { bagażik 'Gapäckraum' } & \text { - bagaż 'Gepäck' } \\ \text { swiecznik 'Kerzenleuchter' } & \text { - swieca 'Kerze' } \\ \text { czajnik 'Teekessel' } & \text { - czaj '(slav.) Tee' } \\ \text { (1i) pilnik 'Feile' } & \text { - piza 'Säge' } \\ \text { odbiornik 'Empfänger(=Radio)' } & \text { - odbior 'Empfang' } \\ \text { chwytnik 'Pinzette' } & \text { - chwyt 'Griff' }\end{array}$

Im Falle von czajnik handelt es sich aller Wahrscheinlichkeit nach um ein tschechisches oder russisches Lehnwort; diese Bezeichnung für 'Teekessel' haben fast alle slavischen Sprachen gemeinsam. Da im Polnischen - im Unterschied 24 allen anderen slavischen Sprachen - für 'Tee' nicht czaj, sondern herbata (lat. herba) gilt, wäre die polnische Ableitung herbatnik; diese gibt es auch, doch mit der Bedeutung 'Teegebăck, Keks'. Die wörter pilnik und piza haben die wurzel mit dem verb pizowac 'sägen' gemeinsam, odbiornik und odbior mit odbierac 'abnehmen, wegnehmen, in Empfang nehmen', chwytnik und chwyt mit chwycic 'fassen, greifen'.

Die wörter $(117(1))$ unterscheiden sich von denen in (117(11)) dadurch, daB für die erstgenannten das objekt ihrer 'Funktion', 
für die anderen dagegen die nähere Bezeichnung der 'Funktion' selbst als unmarkierte Stamm-Repräsentation gelten. Die Paraphrasen können folgendermaBen lauten: pudezko od pior, ozowkow, rqczek etc. 'Kästchen für Federn, Bleistifte, Federhalter etc.' für $(117(i))$, für (117(ii)) dagegen: aparat do odbioru /audycji radiowych/ 'Gerät für den Empfang (von Rundfunksendungen)' oder narzedzie do pizowania 'Werkzeug zum Feilen'. Daß es sich beim Wortstamm in $(117(1 i))$ um ein deverbales Nomen handelt, geht $u$. a. daraus hervor, daß ein verbaler Wortstamm *pizownik ergäbe. Die 'Funktion' der Nomina $(117(1))$ entspricht also einem 'Festgelegtsein' im Sinne von 'Zugehörigkeit', die 'Funktion' der Nomina $(117(1 i))$ dagegen einem Festgelegtsein' im Sinne von 'Instrumentalität' . 70

Für (117) gilt folgende AF-Regel:

$(A F 2.4 .2) K \Rightarrow\{+*, f i k+\}_{N} /\{+\emptyset+\}_{N}$

$$
\left[\begin{array}{c}
- \text { BELEBT } \\
+ \text { BESTIMMTSEIN } \\
{\left[\begin{array}{l}
+ \text { ZUGEHORIGKEIT }] \\
{[+ \text { INSTRUMENTALITÄ }]}
\end{array}\right.} \\
+ \text { MASKULINUM }
\end{array}\right\}
$$

Für die Bildung der Maskulina (117) läßt sich zusammenfassend folgendes feststellen:

(117.1)(a) Die Maskulina (117) werden von Nominalstämmen gebildet; in (117(i)) wird der Wortstamm durch ein Wurzelnomen, in $(117(i i))$ dagegen wird der Wortstamm durch ein deverbales Nomen repräsentiert;

(b) Bezüglich ihrer 'Funktion' entsprechen die Maskulina (117(i)) der 'Zugehörigkeit', die Maskulina (117(ii)) dagegen der 'Instrumentalităt' ;

(c) Im Gegensatz zu den Maskulina (116) sind sie bezüglich [ \pm RÄUMLICH] unmarkiert, desgleichen bezüglich [ \pm BELEBT] des Wortstammes;

(d) Für die phonologische Endableitung gilt u.a. (PL26).

Die K-Repräsentation $\left\{t^{*}, \hat{h} i k+\right\}_{N}$ wird auch bei der Bildung einer groBen Anzahl von denominalen Berufs- bzw. Amtsbezeichnungen zugeordnet, ${ }^{71}$ so z.B.: 
(118) (1) raemieslnik 'Handwerker rolnik 'Landwirt' prawnik 'Jurist' hutnik 'Hüttenarbeiter' gornik 'Bergmann' urzednik 'Beamter' czesnik 'Mundschenk' stolnik 'TruchseB'
- raemiosto 'Handwerk'

- rola 'Acker'

- prawo 'Recht'

- huta 'Hütte'

- gora 'Berg'

- urzqd 'Amt'

- czasza 'Weinpokal'

- stbz 'Tisch'

Die Kennzeichnung der Maskulina (118) als denominal ist insofern nicht ganz eindeutig, da die meisten von ihnen, wenn nicht gar alle, ebenso Adjektiva als Ausgangswörter haben könnten. Wenn hier dennoch von denominalen Nomina gesprochen wird, so geschieht dies aus mehreren Gründen.

Die Adjektiva urzedny oder gorny z.B. Werden im Gegenwartspolnisch entweder kaum oder - und dies im Fall von gorny - mit einer dem 'Bergmann' sehr entfernten Bedeutung gebraucht, nämlich zur Kennzeichnung eines Objekts, das sich auf einer bestimmten 'Höhe' befindet, das 'höher' steht, das 'erhaben' ist. Während unter urzedny einzig SW auf Maczyfiskis Lexicon Latino-Polonicum (Königsberg 1564) verweist. Wollte man hier vom Adjektiv ausgehen, so galte das heutige urzedowy 'Amts-', und dies ergabe das heute nicht mehr gebräuchliche *urzedownik.

Der denominale Ursprung der Maskulina (118) läßt sich synchronisch gesehen auch anders rechtfertigen, nämlich dadurch, daß diese Maskulina in Opposition zu den mit Sicherheit deadjektivischen Maskulina obzudnik 'Lügner' von obzudny 'verlogen' oder beswstydnik 'Schamloser' von bezwstydny 'schamlos' gesehen werden. Sie stehen in Opposition zu den ebenfalls deadjektivischen Maskulina vom Typ zakonnik 'Ordensmann', und dies im Hinblick auf die Bildung der femininen Pendants. Wahrend die femininen Pendants zu (118) z.B. urzedniczka 'Beamtin' oder rolniczka lauten, wird die 'Ordensfrau' bzw. 'Nonne' durch zakonnica ausgedrückt: das Femininum *akonniczka ist nicht möglich.

Da in (118) 'Berufe' bzw. 'Nicht-Berufe' bzw. 'Xmter' ebenso wie in (108) gebildet werden, stellt sich die Frage, wo der Unterschied zwischen (118) und (108) begründet liegt, der die unterschiedlichen $K$-Repräsentationen rechtfertigen würde, zumal in (118) wie in (108) denominale Nomina vorliegen. Im Gegensatz zu (108) liegen in (118) Maskulina vor, die nicht den einzelnen 
'Beruf', sondern eine bestimmte 'Berufsgruppe' bezeichnen; denn als 'Handwerker' - rzemieslnik kann sowohl ein 'Tischler' -stoLarz als auch ein 'Korbflechter' - koszykarz, als 'Arbeiter' - robotnik von robota 'Arbeit' kann sowohl ein 'Dreher' - tokarz als auch ein 'Wollbereiter' - vezniarz, als 'Beamter' bzw. 'Angestellter' - urzednik kann sowohl ein 'Sekretär' - sekretarz als auch ein 'Bibliothekar' - bibliotekarz usw. gelten. Deshalb wird für die Maskulina (118) das Merkmal [ \pm BERUFSGRUPPE] angenommen, wobei [- BERUFSGRUPPE] für 'Amt' u.ä. steht. Als Paraphrasen gelten u.a.: Ten, co sig trudni rzemioszem 'Einer, der sich im Handwerk betätigt'; $T e n$, co pracuje na roli 'Einer, der auf dem Acker arbeitet'; Ten, co pracuje w urzedzie 'Einer, der im Amt arbeitet'....; dies für $(118(i))$, für (118 (ii)) dagegen gelten: Ten od czaszy 'Einer, der für den Weinpokal zuständig ist'; Ten od stozu 'Einer, der für die Tafel zuständig ist'. Die Paraphrasen veranschaulichen die unterschiedlichen 'Funktionen' beider Worttypen, nämlich indem (118(1)) das Merkmal der 'Bestimmung' im Sinne von 'Tätigkeit' - vgl. (108) - zugeordnet werden kann, (118(ii)) dagegen das Merkmal des 'Bestimmtseins' im Sinne von 'Zugehörigkeit'; denn urzednik ist nach dem 'Ort' seiner 'Tätigkeit' benannt, stolnik dagegen nach dem 'Festegelegtsein' gegenüber dem 'Objekt' seiner 'zugehörigkeit'

Für (118) gilt folgende AF-Regel:

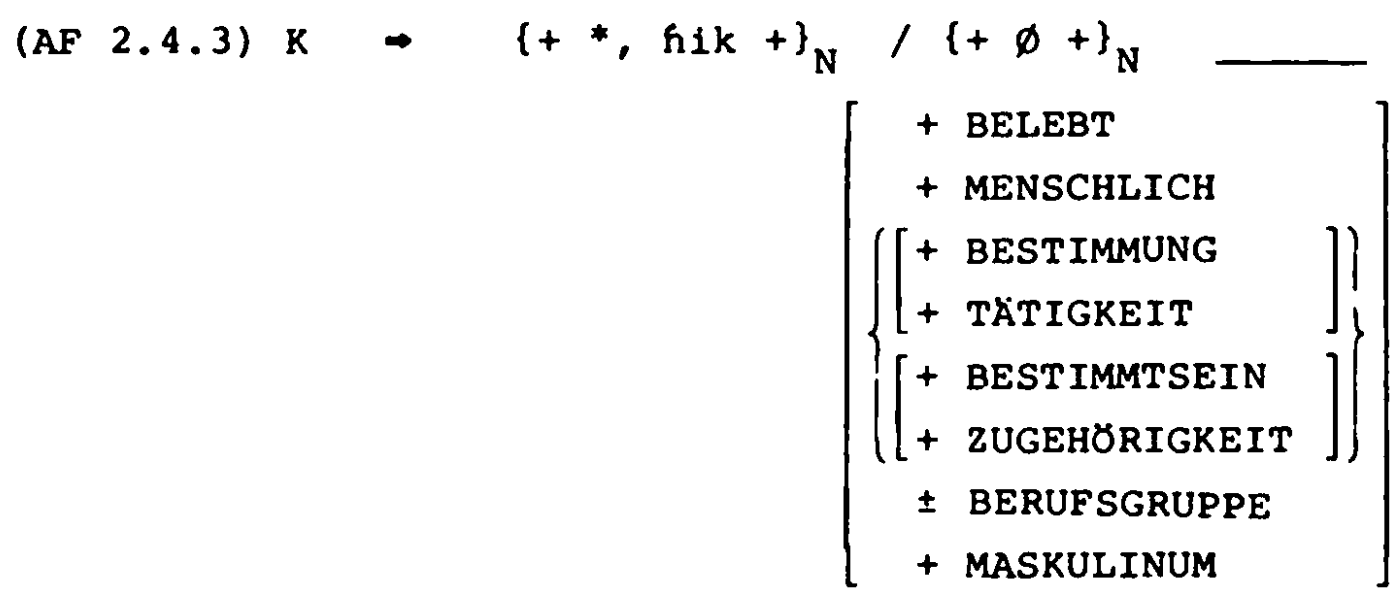

Zusammenfassend läBt sich zur Bildung der Maskulina (118) folgendes feststellen:

(118.1)(a) Die Maskulina (118) sind ausschlieblich denominal;

(b) Die Maskulina (118(i)) sind bezüglich 'Tätigkeit' 
gegenüner den Maskulina (118(1i)) markiert; die Maskulina (118(ii)) dagegen sind gegenüber den Maskulina (118(i)) bezüglich 'Zugehörigkeit' markiert;

(c) Gegenüber den Maskulina (108) sind die Maskulina (118(1)) bezüglich 'Berufsgruppe' markiert, derzufolge auch eine andere $\mathrm{K}$-Repräsentation zugeordnet wird;

(d) Gegenüber den Maskulina (117) sind die Maskulina (118(1)) bezüglich der 'Bestimmung' und der 'Belebtheit', die Maskulina $(118(11))$ bezüglich der 'Belebtheit' markiert;

(e) Für die phonologische Endableitung gelten entweder (PL8)-(PL15) oder (PL26), je nach stammauslaut.

Die bezüglich Genus unmarkierten Maskulina (118(1)) haben ihre markierten femininen Pendants. Die femininen Pendants (119) unterscheiden sich von den Maskulina (118(1)) allein durch das Genus und die mit dem Genus assozilerte $K$-Repräsentation $\{+* k+\}_{N}$.

$\begin{array}{ll}\text { urzedniczka 'Beamtin' } & \text { - uragdnik } \\ \text { rolnicaka 'Landwirtin' } & \text { - rolnik } \\ \text { prawnicaka 'Juristin' } & \text { - prawnik } \\ \text { gorniczka 'Grubenarbeiterin' } & \text { - gornik } \\ \text { hutnicaka 'Hüttenarbeiterin' } & \text { - hutnik } \\ \text { raemieslniczka 'Handwerkerin' } & \text { - ramiemlnik }\end{array}$

Die Bildung der Feminina (119) bleibt - in der Regel - den 'belebten' femininen Pendants zu (118(i)) vorbehalten; dies im Gegensatz zu (113), wo sowohl 'belebte' als auch 'nicht belebte' Feminina gebildet werden konnen. Im Falle von cukiernik - cukierniczka - cukiernica 'Zuckerbăcker - zuckerbăckerin/(DIM)Zukkerdose - zuckerdose' 1st cukiernicaka mit der Bedeutung 'zukkerbăckerin' von cukiernik 'Zuckerbăcker', cukierniczka mit der Bedeutung einer diminutivierten 'Zuckerdose' dagegen von cukiernica 'Zuckerdose' gebildet; im ersten Fall liegt nămlich Nominalisierung - wie in (119) - vor, im zweiten Fall dagegen Diminutivierung, wobei die morphonemische $\mathrm{K}$-Repräsentation der morphonemischen EV-Repräsentation gleicht.

Als Voraussetzung für die Bildung der Feminina (119) muB also die Bildung der Maskulina (108(1)) berücksichtigt werden, d.h. die AF-Regel (AF 2.4.3). Da die Maskulina (118(ii)) für die aufzustellende AF-Regel nicht in Frage kommen - denn die femininen Pendants haben die Bedeutung von 'Ehefrau', vgl. cze\&nikowa 
'Frau des Mundschenks' - , gelten als Voraussetzung die Merkmale [+ MENSCHLICH; - BESTIMMTSEIN; + MASKULINUM ].

Für (119) gilt folgende AF-Regel:

(AF 2.4.4)

$$
\begin{aligned}
& {\left[\begin{array}{l}
+ \text { MENSCHLICH } \\
- \text { BESTIMMTSEIN } \\
+ \text { MASKULINUM }
\end{array}\right]} \\
& K \rightarrow\{+* 8 k+\}_{N} /\{+*, \text { fik }+\}_{N} \\
& {\left[\begin{array}{l}
+ \text { BELEBT } \\
+ \text { MENSCHLICH } \\
+ \text { BESTIMMUNG } \\
+ \text { TATIGKEIT } \\
+ \text { BERUFSGRUPPE } \\
+ \text { FEMININUM }
\end{array}\right]}
\end{aligned}
$$

Zusammenfassend läßt sich zur Bildung der Feminina (119) folgendes feststellen:

(119.1)(a) Die Feminina (119) sind von den Maskulina (118(i)) gebildet und tragen - außer dem Genus - die gleichen Merkmale wie (118(i)):

(b) Gegenüber den Maskulina $(108(i))$ sind die Feminina (119) bezüglich Genus sowie bezüglich der K-Repräsentation markiert;

(c) Die in (AF 2.4.4) zugeordnete feminine K-Repräsentation muß von der morphonemisch gleichen EV-Repräsentation unterschieden werden, da in (119) eine Nominalisierung und keine Diminutivierung stattfindet; (d) Für die phonologische Endableitung gilt u.a. (PL8).

Denominale Nomina liegen auch in (120) vor; es gibt nämlich keine Adjektiva, wie z.B. *puderny, *cukierny, *papierosny, sondern nur pudrowy, cukrowy, papierosowy 'Puder-, Zucker-, zigaretten-'. Wollte man also die Feminina (120) vom Adjektiv bilden, so hiesse es *pudrownica, *cukrownica, papierosownica.
(120) (i) pudernica 'Puderdose' cukiernica 'Zuckerdose'
- puder 'Puder'
papieroßnica 'zigarettenetui'
- cukier 'Zucker'
obdasznica 'Dachrinne'
- papieros 'zigarette'
kostnica
'Leichenhaus'
- obdach 'Obdach'
- koßá 'Knochen'

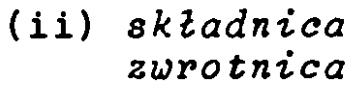
'Lagerraum'
'Weiche'
- skzad 'Lager'
- zwrot 'Unkehr, Wechsel' 
Die Trennung zwischen $(120(i))$ und $(120(i i))$ ist wiederum durch die inhärenten Merkmale bedingt, die durch folgende paraphrasen verdeutlicht werden können: Für (120(i)) gelten: naczynie od pudru, od cukru, od papierosow 'Gefäв für Puder, für zucker, für Zigaretten'; rynna obdachu 'Rinne des Daches'72; für (120 (ii)) gelten dagegen: pomieszczenie do skzadania 'Raum für das Sammeln, Zusammenlegen'; urzqdzenie do zwrbcenia - vgl. SW 'Einrichtung für das Umkehren, Wechseln (von Waggons)'. Im Falle von kostnica gelten sowohl naczynie od kosci 'Gefäß für (verbrannte) Knochen = Urne' als auch izba od kosci zmarzych

' (wörtl.) Leichenknochenstube'; vgl. SW. Während es sich also in (120(i)) um Wortstämme handelt, die durch Wurzelnomina repräsentiert sind, werden die Feminina in (120(ii)) von deverbalen Nominalstämmen gebildet; demnach gilt für die erstgenannten 'Zugehörigkeit', für die anderen dagegen 'Instrumentalität' . Bezüglich u.a. dieser Merkmale entsprechen diese Feminina den Maskulina (117); im SW wird auch piornica neben piornik mit der gleichen Bedeutung 'Federkasten' gebucht, wenngleich piornica als veraltet gekennzeichnet ist. Es gibt jedoch - in der Regel- keine Möglichkeit, (120) von (117) zu bilden; die Nominalisierungen beider Wortgruppen verlaufen getrennt von einander. Allerdings kann bezüglich der Merkmale festgestellt werden, daB (120) gegenüber (117) bezüglich [ \pm RAuMLICH] markiert ist, denn die meisten Feminina (120) bezeichnen 'Räume'.

Für (120(i)) und (120(ii)) gilt folgende AF-Regel:

$(A F$ 2.5.1)

[ - BELEBT ]

$$
\begin{aligned}
& \mathrm{K} \quad \rightarrow \quad\{+* \text {, fic }+\}_{\mathrm{N}} /\{+\emptyset+\}_{\mathrm{N}} \\
& {\left[\begin{array}{c}
\text { - BELEBT } \\
\quad \text { RAUMLICH } \\
+ \text { BESTIMMTSEIN } \\
\left\{\begin{array}{l}
{[+ \text { ZUGEHORIGKEIT }]} \\
{[+ \text { INSTRUMENTALITÄT }]}
\end{array}\right\} \\
+ \text { FEMININUM }
\end{array}\right\}}
\end{aligned}
$$

Das Merkmal [- BELEBT] als Voraussetzung für (AF 2.5.1) weist darauf hin, daß die Feminina (120) bezüglich dieses Merkmals gegenüber den ebenfalls 'räumlichen' Maskulina (116) markiert sind. 
Für die Bildung der Feminina (120) läBt sich zusammenfassend folgendes feststellen:

(120.1) (a) Die Feminina (120) werden von Nominalstämmen gebildet: in (120(i)) wird der Wortstamm durch ein Wurzelnomen, in (120(1i)) wird der Wortstamm durch ein Deverbativum repräsentiert:

(b) Bezüglich ihrer 'Funktion' entsprechen die Feminina (120(1)) der 'zugehörigkeit', die Feminina (120(11)) dagegen der 'Instrumentalităt' ;

(c) Im Gegensatz zu den Maskulina (117) sind sie bezüglich [ \pm RAUMLICH] markiert;

(d) Im Gegensatz zu den Makulina (116) wird ihr nominaler Wortstamm durch [ - BELEBT] gekennzeichnet;

(e) Für die phonologische Endableitung gelten u.a. entweder (PL8)-(PL15) oder (PL26), je nach stammauslaut.

Denominal ist auch eine Reihe von Feminina, die von maskulinen 'Tieren' gebildet werden, so z.B.:

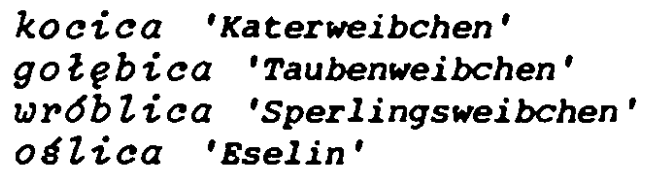

- kot 'Katze'

- gozab 'Taube'

- wrobel 'Sperling'

- osioz 'Esel'

Für den maskulinen Wortstamm in (121) müssen die Merkmale [- MENSCHLICH; + MASKULINUM] als Voraussetzung in die AF-Regel aufgenommen werden. Diese Einschränkung geschieht im Hinblick auf solche wörter, wie z.B. diablica 'Teufelsweib', karlica 'Zwergweib', die von diabez und karzez 'Teufel, Zwerg' gebildet werden und auf den pejorativen Bereich beschränkt zu sein scheinen.

Tierbezeichnungen, die den Auslaut -ik haben, wie z.B. krolik - krolica 'Karnickel-Karnickelweibchen', gehören nicht zu (121). Für diese Feminina mübte eine $K$-Repräsentation $\{+,+\}_{N}$ angenommen werden, um entsprechend (PL13) den Konsonantenwechsel durchfuhren zu können, nämlich:

$$
\begin{aligned}
& \text { (122) } \left.\left[\mathrm{N}_{\mathrm{N}}-\mathrm{krul} \cdot \mathrm{ik}+\emptyset+\right] \rightarrow \mathrm{C}_{\mathrm{N}}[\mathrm{N}-\mathrm{krul} \cdot \mathrm{ik} \emptyset+\mathrm{Q}+]+,+\right] \rightarrow \\
& \left.\rightarrow(\mathrm{DL30}) \rightarrow \mathrm{l}_{\mathrm{N}}-\mathrm{krul}^{\prime} \mathrm{ik},+\right] \rightarrow \\
& \rightarrow(P L 13) \rightarrow\left[_{N}-k r u l ' i c+\right] \quad(k r o l i c a)
\end{aligned}
$$

Ebensowenig gehört samica 'Weibchen' zur Wortgruppe (121), weil 
es - ebenso wie samiec 'Männchen' - zu den Ausnahmen gehört. ${ }^{33}$ Für die regulären Feminina (121) gilt folgende AF-Regel:

(AF 2.6)

$$
\left[\begin{array}{l}
- \text { MENSCHLICH } \\
+ \text { MASKULINUM }
\end{array}\right]
$$

$$
\begin{aligned}
\mathrm{K} \rightarrow\{+* /, \text { ic }+\}_{\mathrm{N}} \quad\left\{\begin{array}{l}
\{+\emptyset+\}_{\mathrm{N}} \\
+ \text { BELEBT } \\
- \text { MENSCHLICH } \\
+ \text { BESTIMMTSEIN } \\
+ \text { ZUGEHORIGKEIT } \\
+ \text { FEMININUM }
\end{array}\right]
\end{aligned}
$$

Das Merkmal des 'Bestimmtseins' im Sinne von 'Zugehörigkeit' ist in (121) anhand folgender Paraphrasierungen festzustellen: samica kota 'des Katers Weibchen': samica wrobla 'des Sperlings Weibchen' usw. In diesen Wortverbindungen liegt der GEN possessivus vor; vgl. dazu Pohl (1983c).

Zusammenfassend läßt sich zur Bildung der Nomina (121) folgendes feststellen:

(121.1)(a) Die Feminina (121) werden von maskulinen Nomina gebildet, die 'Tiere' bezeichnen;

(b) Gegenüber den maskulinen Nomina sind die Feminina (121) bezüglich Genus und K-Repräsentation markiert:

(c) Die femininen Nomina (121) werden durch das inhärente Merkmal der 'Zugehörigkeit' gekennzeicnnet;

(d) Für die phonologische Endableitung gelten u.a. (PL8)- (PL15).

Denominal sind auch die Nomina (123):

$$
\begin{aligned}
& \text { mieszkaniec 'Bewohner' } \\
& \text { powstaniec 'Aufständischer' } \\
& \text { potgpieniec 'Verdamter' } \\
& \text { wygnaniec 'Verbannter' } \\
& \text { zboczeniec 'sittenstrolch' } \\
& \text { odmieniec 'Andersartiger' }
\end{aligned}
$$

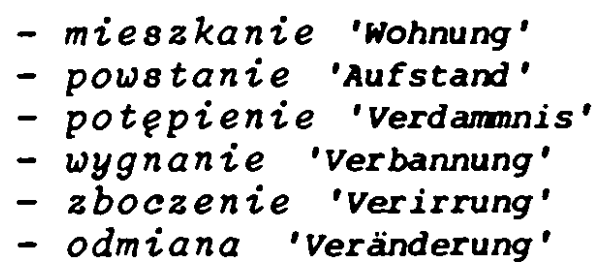

Es könnte der Eindruck entstehen, daB es sich in (123) um Nomina handelt, die vom Partizip gebildet sind. Dieser Eindruck wäre nicht nur deshalb falsch, weil das partizip nicht in der Satz-Basis, sondern im Movement-Teil - vgl. $\$ 5.4 .1$ - gebildet wird, sondern auch deshalb, weil es z.B. keine Partizipien, wie 
*mieszkany oder *powstany gibt. Wollte man vom Partizip ausgehen, so mübte man von mieszkajqcy bzw. powstajqcy, also vom adjektivischen partizip praesentis activi ausgehen, und dies bedeutete, daB die morphonemischen Entsprechungen $z u-j-q c-j e-$ weils getilgt und eine $-n$ - entsprechende morphonemische TF-Repräsentation hinzugenommen werden müBte, um daraus mieszka$-n-i e c$ bzw. powsta-n-iec zu bilden.

Die Paraphrasierungen der Maskulina (123) lauten z.B. ten, co wynajmuje, posiada mieszkanie 'Einer, der eine wohnung mietet, besitzt'; ten, co uczestnicay w powstaniu 'Einer, der am Aufstand teilnimmt'; ten, co nosi skutki potepienia, wygnania, zboczenia, odmiany 'Einer, der die Folgen der Verdammis, der Verbannung, der Verirrung, der Veränderung trägt'. In allen Fällen wird der Wortstamm durch das unmarkierte deverbale Nomen bestimnt, das in wörtern wie powstaniec dem 'Träger' im sinne von 'Tätigkeit' zugeordnet wird, in Wörtern wie wygnaniec dagegen Im Sinne von 'Zustand'; vgl. (95). Für beide gilt das Merkmal der 'Bestimmung' .

Fur die Maskulina (123) gilt folgende AF-Regel:

$$
\begin{aligned}
& \text { (AF 2.7.1) }\{+a \text { h }+\}_{N} \\
& \mathrm{~K} \quad \rightarrow \quad\{+/, 8 c+\}_{\mathrm{N}} /\{+* /, \mathrm{eh}+\}_{\mathrm{N}} \\
& \{+\varnothing+\}_{N} \\
& {\left[\begin{array}{c}
+ \text { BELEBT } \\
+ \text { MENSCHLICH } \\
+ \text { BESTIMMUNG } \\
{[+ \text { TATIGKEIT }]} \\
{[+ \text { ZUSTAND }]} \\
+ \text { MASKULINUM }
\end{array}\right\}}
\end{aligned}
$$

Einigen Maskulina in (123) entsprechen auch Feminina, die jedoch nicht von diesen Maskulina gebildet werden. Dazu gehören u.a. mieszkanka 'Einwohnerin'; powstanka 'Aufständische'; wygnanka 'Verbannte'. Für diese Feminina gilt der gleiche deverbale Nominalstamm wie für die Maskulina, nur werden die Feminina durch Hinzufügung einer anderen K-Repräsentation, nämlich durch $\{+* 8 \mathrm{~K}+\}_{\mathrm{N}}$ gebildet, das dem deverbalen Nominalstamm $z \mathrm{u}-$ gefugt wird. Die inhärenten Merkmale sind die gleichen wie in (AF 2.7.1) bis auf das Genus. 
Zur Bildung der Maskulina (123) läBt sich zusammenfassend folgendes feststellen:

(123.1)(a) Die Maskulina (123) werden von deverbalen Nomina gebildet;

(b) Die inhärenten Merkmale des deverbalen Nominalstammes kennzelchnen die 'Funktion' entweder im Sinne von 'Tätigkeit' oder im Sinne von 'Zustand';

(c) Die femininen Entsprechungen werden nicht vom jeweiligen Maskulinum gebildet; für diese gilt zwar der gleiche deverbale Nominalstamm, doch eine andere $\mathrm{K}-$ -Repräsentation;

(d) Die deverbalen Nominalstämme werden entsprechend (AF 4.3.1) sowie (AF 4.4.2 f.) gebildet;

(e) Für die phonologische Endableitung gelten u.a. (PL15) und (PL16).

Die K-Repräsentation $\{+* /, 8 \mathrm{C}+\}_{\mathrm{N}}$ gilt auch für die 'nicht belebten' Maskulina vom Typ:

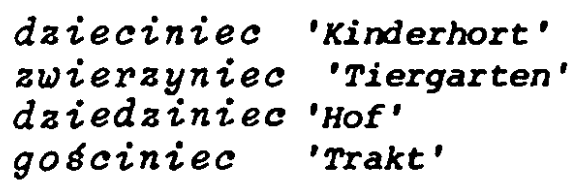

- gobcina'(u.a.)Bewirtung'

2u (124) gehort auch sierociniec 'Waisenhaus', ein Wort, das vom Diminutiv sierocina 'Waise' gebildet wird, obgleich dieses Diminutiv in den wörterbüchern nicht gebucht ist; mit daieciniec ist dieses Diminutiv ein Beispiel für die Einbeziehung des EV - vgl. $\S 6.2 .1 .6$ - in die Nominalisierung. ${ }^{74}$ In der aufzustellenden AF-Regel für (124) wird demnach $\{+* /$, in +$\}$ sowohl durch $\mathrm{N}$ als auch durch EV gekennzeichnet. Wollte man in (124) von den Ausgangswörtern goß́ 'Gast', dziecig 'Kind', zwierze 'Tier', sierota 'Waise' ausgehen, so müBte einerseits dziedziniec ausgeschlossen, anderseits ein TF angenommen werden, der die gleiche morphonemische Repräsentation hätte wie der nominale TM bzw. wie EV; dem widerspräche jedoch die Feststellung, daB TF - vgl. $\S 6.2 .1 .2$ - ausschlieblich phonologisch bedingt ist, denn die vordere Grenze solch eines TF enthielte einen Konsonantenwechsel, der stets mit der Bedeutung assozilert wird.

Dem Nominalstamm der Maskulina (124) liegen Feminina zugrunde, die dem $z u$ bildenden Nomen im sinne von 'Zugehörigkeit' zuge- 
ordnet werden, so z.B. in ogrod/dom dla daieciny 'Garten/Haus für das Kind'; ogrod/park dla zwierzyny 'Garten/Park für das Getier'.

Für (124) gilt folgende AF-Regel:

(AF 2.7.2) [+ FEMININUM]

$\mathrm{K} \quad-\{+* /, 8 \mathrm{C}+\}_{\mathrm{N}} /\{+* /, \text { in }+\}_{\mathrm{N} / \mathrm{EV}}$

$$
\left[\begin{array}{l}
- \text { BELEBT } \\
+ \text { RAUMLICH } \\
+ \text { BESTIMMTSEIN } \\
+ \text { ZUGEHORIGKEIT } \\
+ \text { MASKULINUM }
\end{array}\right]
$$

Zusammenfassend läBt sich für die Bildung der Maskulina (124) folgendes feststellen:

(124.1) (a) Die Maskulina (124) werden von Feminina gebildet, unter denen auch Diminutiva sein können;

(b) Die Maskulina (124) bezeichnen 'Răume', die bezüglich 'Bestimmtsein' im Sinne von 'Zugehörigkeit' gekennzeichnet sind;

(c) Für die phonologische Endableitung gelten u.a. (PL15) und (PL16).

Von den 'belebten' Maskulina (108) und (118) werden folgende Neutra gebildet:

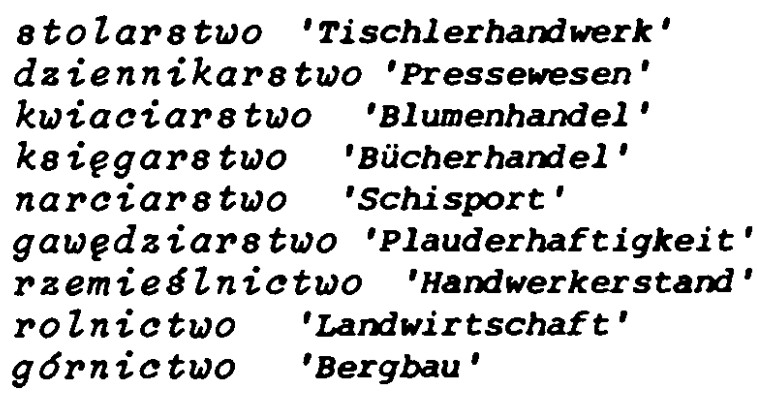

- stolarz

- dziennikarz

- kwiaciarz

- ksiegarz

- narciarz

- gawedziarz

- raemieblnik

- rolnik

- gornik

In (125) handelt es sich um Neutra mit mehreren Bedeutungen: gónictwo z.B. bezeichnet 1) den Industriezweig, der Kohle fördert und verarbeitet; 2) den wissenschaftszweig; 3) die Tătigkeit des Bergbaus; ksiggarstwo bezeichnet 1) den Buchhandel; 2) die Buchhändlerschaft; rzemie\&lnictwo bezeichnet 1) die Tätigkeit des Handwerks; 2) den Handwerkerstand; u.s.w. Um diesen unterschiedlichen Bedeutungen Rechnung zu tragen, wird zwischen 
[ \pm TRAGER] unterschieden; denn der 'Buchhandel' wird im Gegensatz zur 'Buchhändlerschaft' durch [- TRÄGER] - vgl. (90) - gekennzeichnet; es handelt sich nämlich im Falle von 'Buchhandel' allein um die 'Funktion' im Sinne der Definition (95), während die 'Buchhändlerschaft' den 'Träger' dieser 'Funktion' bezeichnet. Ein problem stellen die inhärenten Merkmale (92) insofern dar, als es sich hier um 'nicht zählbare' Appellativa handelt, denen im Falle der Bedeutung 'Buchhändlerschaft' das Merkmal [+ BELEBT] zugeordnet werden müßte, dieses aber nur mit 'zählbaren' Objekten verbunden ist. Um (92) nicht ergänzen zu müssen, wird bezüglich [+ TRÄGER] das Merkmal [ \pm KOLLEKTIVUM] stattdessen zugeordnet. Die 'Buchhändlerschaft' oder die 'Handwerkerschaft' wäre demnach durch die Merkmale [+ TRÄGER; + KOLLEKTIVUM] gekennzeichnet, der 'Industriezweig' oder der 'Handel' durch [+ TRAGER; - KOLLEKTIVUM], die 'Tätigkeit' selbst dagegen durch [- TRÄGER; - KOLLEKTIVUM], wobei in allen drei Fällen das Merkmal [+ FUNKTION] gilt; für alle Bedeutungen gilt auch [- INDIVIDUATIVUM].

Für die Neutra (125) gilt folgende AF-Regel:

(AF 2.8.1)

$$
\begin{aligned}
& {\left[\begin{array}{l} 
\pm \text { BERUF } \\
\pm \text { BERUFSGRUPPE } \\
+ \text { MASKULINUM }
\end{array}\right]}
\end{aligned}
$$

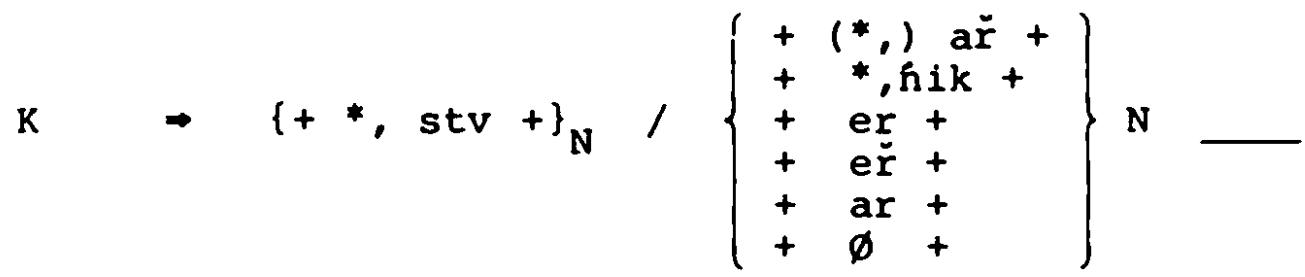

$$
\begin{aligned}
& {\left[\begin{array}{l}
+ \text { FUNKTION } \\
\pm \text { TRAGER } \\
- \text { INDIVIDUATIVUM } \\
\pm \text { KOLLEKTIVUM } \\
+ \text { NEUTRUM }
\end{array}\right]}
\end{aligned}
$$

In (AF 2.8.1) werden nicht nur die Maskulina (108) und (118), sondern auch die Maskulina (104), (105) und (106) einbezogen. Neben den Feminina (113) und (114) - wie z.B. stolarka und stoLarnia - stellen die Neutra (125) eine dritte Möglichkeit dar, von 'belebten' Maskulina, die eine 'Tätigkeit' bezeichnen, andere Nomina $z u$ bilden. Im Hinblick auf die Bedeutung sind jedoch 
die Neutra (125) vergleichsweise an stärksten differenziert. Zusamenfassend läBt sich zur Bildung der Neutra (125) folgendes feststellen:

(125) (a) Die Neutra (125) werden von den Maskulina (104), (105), (106). (108) und (118) gebildet; sie werden auch von Wurzelnamina gebildet, z.B. snob - snobstwo 'Snob-Snobismus, "Snobschaft"';

(b) Gegenüber den Maskulina sind die Neutra (125) sowohl bezüglich Genus als auch bezüglich der K-Repräsentation markiert;

(c) Als 'Trăger' einer 'Funktion' sind die Neutra (125) gegenüber den Maskulina bezüglich [+ KOLLEKTIVUM] markiert; sonst sind sie bezüglich [- TRAGER] markiert;

(d) Gegenuber den ebenfalls demaskulinen Nomina (113), die entsprechend (AF 2.2.2) affiglert werden, und (114) sind die Neutra u.a. bezüglich [- INDIVIDUATIVUM] markiert:

(e) Fur die phonologische Endableitung gilt u.a. (DL28);

(f) Für die K-Reprăsentation in (AF 2.8.1) könnte eine Vokal-Null-Alternation angenommen werden, die z.B. für die Diminutivierung - vgl. paritwo - panstewko 'staat- kleiner staat'75 - benötigt wille.

\subsection{DEADJEKTIVISCHE NOMINA}

Die deadjektivischen Nomina werden gemäB Regel (DER 3) gebildet. Sie bezeichnen u.a. 'Trăger' von 'Eigenschaft' und 'Zugehörigke1t'. 'Răume', 'Fortbewegungsmittel' sowle 'Instrumente'. Für die aufzustellenden AF-Regeln werden die Merkmale (107) impliziert.

In (118) wurden einige Nomina mit der Begründung - vgl. S. 131 ausgeschlossen, das es sich trotz Ubereinstimmung in der $\mathrm{K}-\mathrm{Re}-$ präsentation un deadjektivische Nomina handelt, was dadurch erkennbar 1st, das die Blldung der femininen Pendants eine andere K-Repräsentation erfordert. Bel diesen Nomina handelt es sich - ebenso wie in (118) - um die Bezeichnung keines einzelnen 'Berufes', sondern in den meisten Fallen um die Bezeichnung einer 'Berufsgruppe'. Diese Nomina sind $z$.B. 
(126) (i) robotnik '(phys.)Arbeiter'

chazupnik 'Häusler'

zakonnik 'Ordensmann'

koZchoznik 'Xolchosbauer'

ulicanik 'strabenjunge'

(11) robotnica 'Arbeiterin' chazupnica 'Häuslerin' zakonnica 'Nonne'

kozchoźnica 'Kolchosbäuerin'

ulicanica 'straBenmädchen'
- robotny 'eifrig....'

- chaZupny 'Häusler-'

- zakonny 'Ordens-'

- kozchozny 'Kolchos-'

- ulicany 'straBen-'

- robotnik

- chazupnik

- zakonnik

- kozchosnik

- ulicanik

AuBer robotnik ist all diesen Nomina gemeinsam, daB sie Menschen bezeichnen, die nach dem 'Ort ihres wirkens' benannt sind, robotnik dagegen nach seiner 'Eigenschaft', die auch mit 'arbeitsam' wiedergegeben werden kann. Auch in Falle von robotnik muB von einer diachronischen Erklärung abgesehen werden, zumal auch hier kein Anhaltspunkt für eine andere Ableitung als für die deadjektivische $z u$ finden ist; wăre robotnik deverbal, so muste es *obotownik, von robotowac - vgl. STP -, heiBen; möglich ware eine Ableitung von robota 'Arbeit', dies ebenso in synchronischer sicht, doch widerspricht dem, wie bereits festgestellt, die Bildung des femininen Pendants.

Wie in (118) gilt auch hier das Merkmal [+ BERUFSGRUPPE], denn 2.B. zakonnik kann sowohl ojciec zakonny 'Pater' als auch brat zakonny 'Frater' sein, robotnik vereint ebenfalls zahlreiche 'Berufe' - vgl. S. 132 -, desgleichen der 'Hăusler' oder der 'Kolchosbauer'. Doch wăhrend der 'Arbeiter' nach seiner 'Elgenschaft' benannt ist, gilt fur die anderen 'Zugehorigkeit'. ${ }^{76}$

Wenn für die adjektivische TM-Repräsentation $\{+*, 8 n+\}^{77}$ angenommen wird, so gilt für die Maskulina (126(1)) folgende AF-Regel:

$$
\begin{aligned}
& (\mathrm{AF} 3.1 .1) \mathrm{K} \quad \rightarrow \quad\{+, i k+\}_{\mathrm{N}} /\{+* .8 \mathrm{n}+\}_{\mathrm{A}} \\
& {\left[\begin{array}{c}
+ \text { BELEBT } \\
+ \text { MENSCHLICH } \\
+ \text { BESTIMMTSEIN } \\
\left\{\begin{array}{l}
{[+ \text { EIGENSCHAFT }]} \\
{[+ \text { ZUGEHORIGKEIT }]}
\end{array}\right\} \\
+ \text { BERUFSGRUPPE } \\
+ \text { MASKULINUM }
\end{array}\right\}}
\end{aligned}
$$

Für die Bildung der femininen Pendants (126(ii)) gibt es zwei 
Möglichkeiten, $K$ morphonemisch zu repräsentieren, entweder durch $\{+* /, \text { ic }+\}_{N}$ oder durch $\{+,+\}_{N}$. Im Falle der erstgenannten $\mathrm{K}-$ -Repräsentation wïrden die Feminina nicht von den Maskulina gebildet, sondern, wie die Maskulina, vom adjektivischen Wortstamm im Falle der anderen K-Repräsentation würden dagegen die Feminina (126(ii)) von den Maskulina (126(i)) so gebildet, das das auslautende $\{k\} \mathrm{zu}\{\mathrm{c}\}$ würde. Gegen die letztgenannte Möglichkeit spricht die Tatsache, daB mit Hilfe der für (126(ii)) aufzustellenden AF-Regel auch solche Feminina von Adjektiven gebildet werden, die kein maskulines pendant haben, nämlich (127). Für die inhärenten Merkmale der Feminina (126(i1)) gelten folgende Wortverbindungen: kobieta robotna 'arbeitsame Frau'; robotnica hazupna ' (etwa)Hausarbeiterin': siostra zakonna 'Ordensschwester'; robotnica kozchozina 'Kolchosarbeiterin'; kobieta ulicana 'Straßenfrau'; demnach gilt hier 'Bestimmtsein' im Sinne von 'zugehörigkeit' sowie [+ BERUFSGRUPPE].

Bezüglich 'Eigenschaft' sind folgende Feminina gekennzeichnet:

$$
\begin{array}{ll}
\text { obzudnica 'Lügnerin' } & \text { - obzudna 'verlogen' } \\
\text { zazdrołnica 'Eifersüchtige' } & \text { - zazdrosna 'eifersüchtig' } \\
\text { bezwstydnica 'Schamlose' } & \text { - bezwstydna'schanlos' } \\
\text { samotnica 'Einsame' } & \text { - samotna 'einsam' } \\
\text { Zadacznica 'liederliches Frauen- } & \text { - ladaczna 'liederlich' } \\
& \text { zimmer' }
\end{array}
$$

Für (127) wird das Merkmal [- BERUFSGRUPPE] angenommen, und für die Feminina (126(i1)) sowie (127) gilt folgende AF-Regel:

$$
\begin{aligned}
& (A F 3.2 .1) K \rightarrow\{+*, i c+\}_{N} /\{+* .8 n+\}_{N} \\
& {\left[\begin{array}{c}
+ \text { BELEBT } \\
+ \text { MENSCHLICH } \\
+ \text { BESTIMMTSEIN } \\
\left\{\begin{array}{l}
{[\text { EIGENSCHAFT }]} \\
{[+ \text { ZUGEHORIGKEIT }]}
\end{array}\right\} \\
\pm \text { BERUFSGRUPPE } \\
+ \text { FEMININUM }
\end{array}\right\}}
\end{aligned}
$$

Zusammenfassend läBt sich zur Bildung der Maskulina (126(i)) sowie der Feminina (126(ii)) und (127) folgendes feststellen:

(126.1)(a) Die Maskulina (126(i)) und die Feminina (126(ii)) so(127.1) wie (127) sind deadjektivisch;

(b) Sowohl die Maskulina als auch die Feminina werden 
durch 'Bestimmtsein' im Sinne von 'Zugehörigkeit' bzw. 'Eigenschaft' gekennzeichnet; für beide gilt auch das Merkmal [ \pm BERUFSGRUPPE];

(c) Die Feminina sind gegenüber den Maskulina bezüglich Genus und bezüglich der K-Repräsentation markiert;

(d) Gegenüber den Maskulina (118) sind die Maskulina (126(i)) bezüglich 'deadjektivisch' markiert;

(e) Gegenüber $(120(i))$ sind die Feminina $(126(i i))$ sowie (127) bezüglich [ - INSTRUMENTALITÄT; + BELEBT] markiert, gegenüber (119) bezüglich 'deadjektivisch'; (f) Für die phonologische Endableitung gilt u.a. (PL15).

Das Merkmal der 'Berufsgruppe' gilt auch für deadjektivische Maskulina, die 'Trăger' bestimmter 'Eigenschaften' oder 'Zugehörigkeiten' sind. Dazu gehören:

(128)(i) naZogowiec 'Süchtiger' uczuciowiec 'Gefühlsmensch' pechowiec 'Pechvogel'

(ii) stocaniowiec 'Werftarbeiter' alwodowiec 'Profi' filmowiec 'Filmer' akordowiec 'Akkordarbeiter' sportowiec 'sportler' fachowiec 'Fachmann'
- naZodowy 'süchtig'

- uczuciowy 'Gefühls-

- pechowy 'Pech-'

- stocaniowy 'Werft-'

- sawodowy 'Berufs-'

- filmowy 'Film-'

- akordowy 'Akkord-'

- sportowy 'sport-'

- fachowy 'Fach-'

Im Gegensatz zu den denominalen Maskulina (123) liegt in (128) keine 'Bestimmung', sondern 'Bestimmtsein' vor; dieses bezüglich der vom Adjektiv-Stamm bezeichneten 'Eigenschaft' bzw. 'zugehörigkeit'. Es könnte angenommen werden, daB diese Maskulina vom ADV gebildet werden, nămlich: ten, co pracuje zawodowo 'Einer, der beruflich arbeitet'; aber wie sollte dann die Paraphrase von filmowiec lauten; *ten, co pracuje filmowo 'Einer, der filmisch arbeitet' ist nicht möglich. Dafür aber: robotnik stoczniowy, zawodowy, akordowy, fachowy 'Werft-, Berufs-, Akkord-, Facharbelter' oder pracownik filmowy 'Filmschaffender' oder aber czZowiek nazogowy, uczuciowy, pechowy 'Sucht-, Gefuhls-, Pech-Mensch'. Es ist anzunehmen, daB diesen Nomina Univerbierungen zugrunde liegen, d.h., daB diese Nomina Ergebnisse von Univerblerungen sind.

Wenn für die TM-Repräsentation $\left\{+\&{ }_{3} v+\right\}_{N}$ angenommen wird, so gilt folgende AF-Regel für die Bildung der Maskulina (128): 
$(A F 3.3 .1) \mathrm{K} \rightarrow\{+* / .8 \mathrm{C}+\}_{\mathrm{N}} /\left\{+\&_{3} \mathrm{~V}+\right\}_{\mathrm{A}}$

$$
\left[\begin{array}{c}
+ \text { BELEBT } \\
+ \text { MENSCHLICH } \\
+ \text { BESTIMMTSEIN } \\
{\left[\begin{array}{l}
+ \text { EIGENSCHAFT }] \\
{[+ \text { ZUGEHORIGKEIT }]} \\
\pm
\end{array}\right\}} \\
+ \text { BERUFSGRUPPE }
\end{array}\right\}
$$

Die gleiche K-Repräsentation haben auch 'nicht belebte' Maskulina, die ebenfalls deadjektivisch sind und die 'Fortbewegungsmittel' bezeichnen; dazu gehören:
(129) iaglowiec 'Segelschiff'
parowiec 'Dampfschiff' torpedowiec 'Torpedoboot' smigzowiec 'Hubschrauber' odrzutowiec 'Düsenjäger'
- zaglowy 'Segel-'
- parowy 'Dampf-'
- torpedowy 'Torpedo-'
- smigzowy 'Propeller-'
- odrzutowy 'Abwurf-'

Die Nomina (129) können ebenfalls als Ergebnisse von Univerbierungen gesehen werden; die dazu gehörenden Wortverbindungen sind: statek iaglowy, statek parowy, statek torpedowy 'segel-, Dampf-Schiff, Torpedoboot'; samolot smigzowy, samolot odrzutowy '(wörtl.) Propeller-, Abwurf-Flugzeug'. Demnach ist das unmarkierte A gegenüber dem markierten $N$ bezüglich 'Bestimmtsein' im Sinne von 'Instrumentalităt' gekennzeichnet.

Für (129) gilt folgende AF-Regel:

$(A F 3.3 .2) K \Rightarrow\{+* / .8 C\}_{N} /\left\{+\&_{3} V+\right\}_{A}$

$$
\left[\begin{array}{l}
- \text { BELEBT } \\
- \text { ABSTRAKT } \\
+ \text { BESTIMMTSEIN } \\
+ \text { INSTRUMENTALITÄT } \\
+ \text { MASKULINUM }
\end{array}\right]
$$

Zusammenfassend läßt sich für die Bildung der Maskulina (128) und (129) folgendes feststellen:

(128.1) (a) Die Maskulina (128) und (129) werden von Adjektiven (129.1) gebildet; wobei für (128(1)) 'Bestimmtsein' im Sinne von 'Eigenschaft', für (128(ii)) 'Bestimmtsein' im Sinne von 'Zugehörigkeit' und für (129) 'Bestimmtsein' im sinne von 'Instrumentalität' gelten; 
(b) Für die Maskulina (128(ii)) gilt das Merkmal der 'Berufsgruppe' sowohl im Sinne von 'Eigenschaft' als auch in Sinne von 'Zugehörigkeit';

(c) Für die Maskulina (129) gilt das Merkmal [+ FORTBEWEGUNGSMITTEL] im Sinne der 'Instrumentalität' ;

(d) Gegenüber (129) ist (128) u.a. bezüglich [+ BELEBT] markiert, desgleichen gegenüber den Maskulina (124);

(e) Gegenüber den denominalen Maskulina (123) und (124) sind die dadjektivischen Maskulina (129) bezüglich 'Instrumentalităt' neben 'deadjektivisch' markiert; (128) U.a. bezüglich 'deadjektivisch';

(f) Für die phonologische Endableitung der adjektivischen TM-Reprasentation in (AF 3.3.1) und (AF 3.3.2) gilt (PL19);

(g) Für die phonologische Endableitung gelten sonst u.a. (PL15) und (PL16).

Wăhrend die Maskulina (128) und (129) von Relativa unter den Adjektiven gebildet werden, liegen den Maskulina (130) Qualitativa als Wortstamm-Repräsentation zugrunde, nämlich:
(130) malec 'Knirps'
medrzec 'Weiser'
starzec 'Greis'
skqpiec 'Geizhals'
- mazy 'klein'
- mqdry 'klug'
- stary 'alt'
- skqpy 'geizig'

Entsprechend dem in (95) definierten Merkmal [+ EIGENSCHAFT], dies u.a. bezüglich 'Qualität' und/oder 'Quantität', kann in (130) von einer 'Verstärkung' ausgegangen werden, die sowohl die vom Qualitativum bezeichnete 'Eigenschaft' als auch den vom markierten $\mathrm{N}$ bezeichneten 'Trăger' betrifft, so z.B. starzec bardzo madry 'sehr kluger Greis' oder czlowiek bardzo skapy 'sehr geiziger Mensch' U.s.w. 78

Für die Maskulina (130) gilt folgende AF-Regel:

$(A F 3.3 .3) \mathrm{K} \quad \Rightarrow \quad\{+/ .8 \mathrm{C}+\}_{N} /\{+\varnothing+\}_{A}$

$$
\left[\begin{array}{l}
+ \text { BELEBT } \\
+ \text { MENSCHLICH } \\
+ \text { BESTIMMTSEIN } \\
+ \text { EIGENSCHAFT } \\
+ \text { MASKULINUM }
\end{array}\right]
$$


Die deadjektivischen Nomina (130) sind gegenüber (124) und (129) bezüglich [+ BELEBT; + EIGENSCHAFT], gegenüber (123) bezüglich [+ EIGENSCHAFT] und gegenüber (128) bezüglich des durch ein Qualitativum repräsentierten wortstammes markiert.

Reguläre feminine Pendants zu den deadjektivischen Nomina (128) und (129) sind selten. Für (128) kann festgestellt werden, daB die femininen Pendants gegenüber den Maskulina bezüglich [- BELEBT] markiert sind und infolge dessen auch eine andere Bedeutung haben, wie z.B. zawodowka - szkoza zawodowa 'Berufsschule' oder fachowka - szkoza fachowa 'Fachschule'. Für (129) kann festgestellt werden, daß in einigen Fällen das feminine Pendant ebenfalls ein 'Fortbewegungsmittel' bezeichnen kann, so z.B. motorowiec - statek motorowy 'Motorschiff' - motorowka - zodz motorowa 'Motorboot'. Generell herrscht jedoch die Tendenz vor, Feminina auf - $\delta w k-a$ ohne einen Markiertheitsbezug zu den entsprechenden - falls vorhandenen - Maskulina zu bilden.

Zu den femininen 'Fortbewegungsmitteln' gehören u.v.a.:

\begin{tabular}{|c|c|c|}
\hline $\begin{array}{l}\text { zaglowka } \\
\text { motorowka } \\
\text { ciezarowka }\end{array}$ & $\begin{array}{l}\text { 'Segelboot' } \\
\text { 'Motorboot' } \\
\text { 'Lastkraftwagen' }\end{array}$ & $\begin{array}{l}\text { - zaglowy } \\
\text { - motorowy } \\
\text { - ciezarowy }\end{array}$ \\
\hline
\end{tabular}

In der Regel ist das Genus FEMININUM in (131) durch das Genus des markierten Nomen bedingt, das - vgl. $\$ 5.4 .2$ - getilgt und entsprechend durch die $\mathrm{K}$-Repräsentation ersetzt wird; denn die (131) zugrunde liegenden Wortverbindungen sind: $z \sigma d \dot{z}$ (FEM) $\dot{z} a g-$ lowa, Zodí motorowa, ? N (FEM) cifiarowa. Die letztgenannte Wortverbindung widerspricht bereits der Regel, weil hier kein feminines $\mathrm{N}$ vorhanden ist, sondern nur samochod (MAS) 'Auto': zur Lösung dieses Problems siehe $\S 9.3$.

Bezüglich des Genus FEMININUM sowie bezüglich des Merkmals [+ INSTRUMENTALITÁT] lassen sich die Nomina (131) um (132) erweitern, nämlich um Nomina, wie z.B.

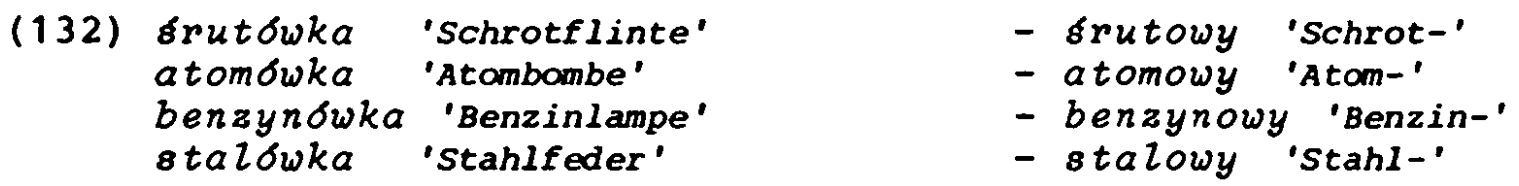

Als Wortverbindungen für (132) gelten: strzelba frutowa 'Schrotflinte': bomba atomowa 'Atombombe': Lampa benzynowa'Benzinlampe' und wiederum ohne entsprechende N-Repräsentation ?N (FEM) 
stalowa 'Stahlfeder'; 'Feder' hat im Polnischen das Genus NEUTRUM, nämlich pioro, wenngleich dieses Nomen für 'Federhalter' steht.

Für die deadjektivischen Nomina (131) und (132) gilt folgende AF-Regel:

$(A F 3.4 .1) \mathrm{K} \quad \rightarrow \quad\{+* 8 k+\}_{N} /\left\{+8_{3} V+\right\}_{A}$

$$
\left[\begin{array}{l}
- \text { BELEBT } \\
- \text { ABSTRAKT } \\
+ \text { BESTIMMTSEIN } \\
+ \text { INSTRUMENTALITATT } \\
\pm \text { FORTBEWEGUNGSMITTEL } \\
+ \text { FEMININUM }
\end{array}\right]
$$

Die 'Fortbewegungsmittel' in (131) sind also nur bezulglich des Genus und der K-Repräsentation gegenuber (129) markiert, die 'Nicht-Fortbewegungsmittel' zusätzlich durch [- FORTBEWEGUNGSMITTEL].

Zusammenfassend läBt sich zur Bildung der Feminina (131) und (132) folgendes feststellen:

(131.1)(a) Die Bildung der deadjektivischen Feminina (131) und (132.1) (132) impliziert nicht die Bildung der deadjektivischen Maskulina (129);

(b) Den meisten Feminina (131) und (132) liegen Wortverbindungen aus $\mathrm{N}$ und $\mathrm{A}$ zugrunde, die univerbiert werden können;

(c) Das Genus des markierten, zugrundeliegenden Nomen entspricht in der Regel dem Genus des univerbierten Nomen ;

(d) Gegenuber den Maskulina (129) sind die Feminina (131) und (132) bezulglich [- BELEBT] sowie [+ FEMININUM] markiert:

(e) Für die phonologische Endableltung der TM-Repräsentation gilt (PL19); fur die phonologische Endableitung sonst gelten u.a. (PL8) und (PL16).

Neben den denominalen 'Raumbezeichnungen', die mittels der $\mathrm{K}$ Repräsentation $\left.\left\{+^{*}, 8\right\}+\right\}_{N}$ gebildet werden - vgl. (114) und (115)- 
gibt es auch solche, die von Relativa gebildet werden. Dazu gehören u.v.a.:

(133) maszynownia 'Maschinenhalle' weglownia 'Kohlendeck/-halle' wagonownia 'Waggonreparaturraum' prochownia 'Pulverlager/-fabrik' koksownia 'Kokcrei'
- maszynowy 'Maschinen-'

- weglowy 'Kohle(n)-'

- wagonowy 'waggon-'

- prochowy 'Pulver-'

- koksowy 'Koks-'

Diesen Feminina liegen folgende wortverbindungen zugrunde, einerseits: hala maszynowa, weglowa, wagonowa, prochowa 'Maschinen-, Kohlen-, Waggon-, (SchieB-)Pulver-Halle', ebenso: hala od maszyn, od wegla, od wagonow, od prochu 'Halle für Maschinen, für Kohlen, für Waggons, für Pulver', anderseits: fabryka, co produkuje proch 'Fabrik, die SchieBpulver herstellt - vgl. SW'; fabryka, co produkuje koks 'Werk, das Koks herstellt'. Aus den erstgenannten Wortverbindungen geht hervor, daB das denominale Relativum im Wortstamm nicht das 'Produkt', sondern ein 'zu bearbeitendes', ein 'zu verarbeitendes', ein 'mitarbeitendes' oder 'zu lagerndes' Objekt bezeichnet, nach welchem der 'Raum' benannt ist. Demnach gilt hier 'Bestimmtsein' im Sinne von 'Zugehörigkeit'. In prochownia gilt obendrein, da es auch um das herzustellende 'Produkt' geht, und in koksownia ausschlieblich, 'Bestimmtsein' im Sinne von 'Instrumentalität', ähnlich übrigens wie in (115). Folglich müssen für (133) beide Merkmale als Bedingungen für die zuordnung der $\mathrm{k}$-Repräsentation eingesetzt werden.

Für die Abgrenzung gegenüber (115) sprechen zwei Feststellungen, nämlich 1) die Feminina (133) sind Produkte von Univerbierungen; sie sind gegen $\mathrm{N}-\mathrm{A}$-Verbindungen austauschbar; 2) Werden die $\mathrm{Fe}-$ minina (115) als Produkte von Univerbierungen gesehen, so sind sie gegen $\mathrm{N}-\mathrm{N}$-Verbindungen austauschbar, nämlich: fabryka papieru, cegiez, lakieru 'Papier-, Ziegel-, Lack-Fabrik' sowie przetwornia cukru 'Zuckerverarbeitungsfabrik'. Wären in (115) ebenfalls N-A-Verbindungen möglich, so hieße es: *papierownia, *cegZownia. Demnach sind die Feminina (133) gegenüber den Feminina (115) bezüglich 'deadjektivisch' markiert; der Grund dafür ist auch in der unterschiedlichen Bedeutung der potentiellen Adjektiva für die Nominalstämme in (115) zu sehen; papierowy z.B. bezeichnet die 'Beschaffenheit', also entspräche *fabryka papierowa einer 'Fabrik aus Papier'. 
Für die Bildung der deadjektivischen Feminina (133) gilt folgende AF-Regel:

$(A F 3.5 .1) K \rightarrow\{+* .8 \mathrm{~h}+\}_{N} /\left\{+88_{3} \mathrm{~V}+\right\}_{A}$

$$
\left[\begin{array}{c}
- \text { BELEBT } \\
- \text { ABSTRAKT } \\
+ \text { RAUMLICH } \\
+ \text { BESTIMMTSEIN } \\
\left\{\begin{array}{l}
{[+ \text { ZUGEHORIGKEIT }]} \\
{[+ \text { INSTRUMENTALITAT }]}
\end{array}\right\} \\
+ \text { FEMININUM }
\end{array}\right\}
$$

Zusammenfassend läBt sich zur Bildung der Feminina (133) folgendes feststellen:

(133.1)(a) Die Feminina (133) werden von Adjektiva gebildet, die einen 'Teil', der für den 'Raum' 'bestimmt ist', oder ein 'Produkt', das in diesem 'Raum' hergestellt wird, bezeichnen;

(b) Das 'Bestimmtsein' des vom Femininum bezeichneten 'Raumes' entspricht der 'Zugehörigkeit' und/oder der 'Instrumentalität' ;

(c) Gegenüber den denominalen Feminina sind die Feminina (133) bezüglich 'deadjektivisch' markiert:

(d) Den Feminina (133) liegen N-A-Verbindungen zugrunde;

(e) Für die phonologische Endableitung der TM-Repräsentation gilt (PL19); für die phonologische Endableitung sonst gelten u.a. (PL16) und (DL26).

\section{4. DEVERBALE NOMINA}

Zu don deverbalen Nomina gehören - in der Regel - sowohl solche, die den 'Träger' u.a. einer 'Tätigkeit' bezeichnen, wie z.B. nauczyciel 'Lehrer', als auch die sog. "Verbalwörter", wie z.B. nauka 'Lehre' sowie nauczanie 'Lehren'. Der Unterschied wird durch das Merkmal [ \pm TRAGER] gekennzeichnet, also durch die Unterscheidung zwischen dem 'Verursacher' bzw. 'Veranlasser' und der 'Verursachung' bzw. 'Veranlassung' im sinne (95); m.a.w.: Der Unterschied zwischen nauczyciel und nauka entspricht dem Unterschied zwischen dem 'Funktionstrăger' und der 'Funktion' selbst. 
Als Voraussetzung für die aufzustellenden AF-Regeln gelten u.a. die Merkmale (88) und (94) sowie die Unterscheidung der Verben hinsichtlich ihrer jeweiligen K-Repräsentation.

Es wird zwischen folgenden V-Klassifikatoren unterschieden: ${ }^{79}$

$$
\begin{aligned}
& \{+(* /,) \mathrm{a}+\}_{\mathrm{V}} \text { (szturchac 'schubsen'/ ruszac 'bewegen') } \\
& \{+*, i+\} \mathrm{V} \text { (dusic 'würgen') } \\
& \{+* /, i+\}_{v} \text { (suszyc 'trocknen') } \\
& \{+* /, e+\}_{V}(k r z y c z e c \text { (schreien') } \\
& \{+\quad \tilde{}+\}_{\mathrm{V}} \text { (krzyknqc 'aufschreien') } \\
& \{+\varnothing+\} \mathrm{v} \text { (kzuc 'stechen') } \\
& \{+ \text { ova }+\}_{\mathrm{V}} \text { (pracowac 'arbeiten') }
\end{aligned}
$$

Da es sich im folgenden ausschlieblich um deverbale Nomina handelt, ist allen 'belebten Funktionsträgern' das Merkmal [+ BESTIMMUNG] im sinne von [+ ZUSTAND] oder [+ TÄTIGKEIT] - je nach Verb - gemeinsam; wăhrend die 'nicht belebten Funktionsträger' durch [- zUGEHORIGKEIT] im Sinne von [+ INSTRUMENTALITAT] - vgl.

\begin{tabular}{|c|c|c|}
\hline$(135)(i)$ & $\begin{array}{l}\text { spawacz 'Schweißer' } \\
\text { badacz 'Porscher' } \\
\text { tkacz 'Weber' } \\
\text { odlewacz 'GieBer' } \\
\text { skZadacz 'Setzer' } \\
\text { walcowacz 'Walzwerker' } \\
\text { słuchacz 'Hörer' } \\
\text { biegacz 'Läufer' } \\
\text { działacz 'Aktivist' } \\
\text { tuZacz 'Vagabund' }\end{array}$ & $\begin{array}{l}\text { - spawad 'schweiBen' } \\
\text { - badac 'forschen' } \\
\text { - tkad 'weben' } \\
\text { - odlewad '(ab-)gießen' } \\
\text { - skzadac 'setzen' } \\
\text { - walcowad 'walzen' } \\
\text { - stuchac 'hören' } \\
\text { - biegac 'laufen' } \\
\text { - dziazac 'wirken' } \\
\text { - tuzas sig 'herumirren' }\end{array}$ \\
\hline (ii) & $\begin{array}{l}\text { Zadowacz 'Ladearbeiter/Lader' } \\
\text { Zamacz 'Metteur/Brechmaschine' }\end{array}$ & $\begin{array}{l}\text { - Zadowac 'laden' } \\
\text { - Zamac 'brechen' }\end{array}$ \\
\hline (iii) & $\begin{array}{l}\text { rozpylacz 'Zerstäuber' } \\
\text { rozilewacz 'Sämaschine' } \\
\text { odkurzacz 'Staubsauger' } \\
\text { pogrzebacz 'Schürhaken' }\end{array}$ & $\begin{array}{l}\text { - rozpylad 'zerstäuben' } \\
\text { - rozsiewad 'aussäen' } \\
\text { - odkurzad 'entstauben' } \\
\text { - pogrzebad 'stochern' }\end{array}$ \\
\hline
\end{tabular}
(94) - gekennzeichnet werden.

Fúr die deverbalen Maskulina auf -acz gelten sowohl [+ BELEBT] als auch [- BELEBT]; so bezeichnet 2.B. Zadowacz sowohl den 'Ladearbeiter' als auch die 'Lademaschine'; Zamacz sowohl den 'Metteur' als auch die 'Brechmaschine' bzw. den 'Elsbrecher'. zu den 'Funktionsträgern' dieser Gruppe zählen:

Die Maskulina (135) lassen sich folgendermaBen paraphrasieren: ten, co spawa, co bada, co tka,..., co Zaduje 'Einer, der schwelBt, der forscht, der webt,..., der lädt'. Demnach wird 
für [+ BELEBT] die 'Bestimmung' im Sinne von 'Tätigkeit' angenommen. Entsprechend przyrzqd, co Zaduje, odkurza, rozpyla 'Ein Gegenstand, der lädt, entstaubt, zerstäubt' wird für [- BELEBT] das Merkmal des 'Bestimmtseins' im Sinne von 'Instrumentalität' angenommen. Für die Verbalstämme gilt [+ IMPERFEKTIV].

Es gehört zu den Relikten aus der Wortbildung im Altpolnischen, daB eine Abgrenzung der Nomina (135) von den ebenfalls deverbalen auf $-n i k,-c-a$ und auch teilweise auf -iciel schwer möglich ist; denn im Gegenwartspolnisch gelten noch als bedeutungsgleich 2.B. walcarz, walcowacz, walcownik 'Walzwerker', desgleichen wedrowca, wedrownik 'Wanderer' oder zbawca, zbawiciel 'Erlöser'. Es gibt zwar im Gegenwartspolnisch Fälle, in denen zwischen z.B. kierownik '(Abteilungs)Leiter' und kierowea 'Chauffeur' unterschieden wird, wobei die Möglichkeit besteht, auch hier zwischen 'Berufsgruppe' und 'Beruf' $z$ unterscheiden, wobei allerdings 'Beruf' für kierowca nur im Falle des 'Berufskraftfahrers' zuträfe, doch beträfe diese Unterscheidung Einzelfälle. Daher wird auch in den aufzustellendèn AF-Regeln auf eine nähere Bezeichnung des 'Funktionsträgers' verzichtet.

Die Maskulina (135) werden hauptsächlich von Verbalstämmen mit der TM-Repräsentation $\{+(* /,) \text { a }+\}_{V}$ gebildet; das Maskulinum Zadowacz gehört hier also $z$ den Ausnahmen: desgleichen walcowacz, der allerdings mit walcownik bedeutungsgleich ist.

Für die Maskulina (135) gilt folgende AF-Regel:

$(A F$ 4.1.1)

[+ IMPERFEKTIV ]

$$
\begin{aligned}
\mathrm{K} \rightarrow\{+\mathrm{ac}+\}_{\mathrm{N}} & /\left\{\begin{array}{l}
\{+a+\} \\
(\{+ \text { ova }+\})
\end{array}\right] \\
& {\left.\left[\begin{array}{l}
+ \text { FUNKTION } \\
+ \text { TRAGER } \\
{\left[\begin{array}{l}
+ \text { BELEBT } \\
+ \text { MENSCHLICH } \\
+ \text { TATIGKEIT }
\end{array}\right]} \\
{\left[\begin{array}{l}
\text { BELEBT } \\
+ \text { INSTRUMENTALITAT } \\
+ \text { MASKULINUM }
\end{array}\right]}
\end{array}\right\}\right) }
\end{aligned}
$$


Die Verbalstämme auf -owa- $c$ bestimmen folgende Maskulina:

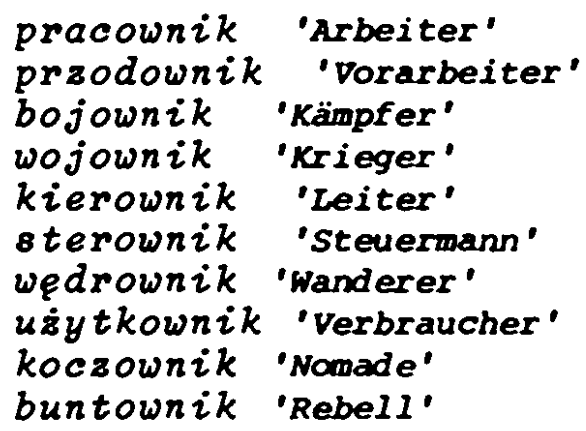

- pracowad 'arbeiten'

- przodowad 'hervorragen'

- bojowal 'kämpfen'

- wojowal 'Krieg führen'

- kierowad 'leiten/lenken'

- sterowac 'steuern'

- wedrowad 'wandern'

- u̇ंytkowad 'verwenden'

- koczowal 'umherschweifen'

- buntowad 'aufwiegeln'

Für die Paraphrasierung gelten u.a.: ten, co pracuje, co przoduje, co bojuje, co wojuje....'Einer, der arbeitet, der hervorragt, der kămpft, der krieg führt,...', wobei für das unmarkierte Verb - vgl. $\$ 5.4 .1$ - nicht die flektierte Fom, sondern der Infinitiv einzusetzen 1st. Da also der Verbalstamn eine 'Tătigkeit bezelchnet, die einem 'belebten Träger' zugeordnet wird, werden die Nomina (136) durch [+ BESTIMMUNG] und [+ TÁTIGKEIT] gekennzeichnet.

Bei der Affigierung der Maskulina (136) wird nur der letzte Vokal der V-Reprasentation - entsprechend (DL29(1)) - getilgt, die Morphonemverbindung fov\} dagegen bleibt erhalten; auf diese Weise kann der Verbalstamm z.B. vom Verbalstamm auf -aC, wie er in (135) verwendet wird, unterschieden werden, un die nominale

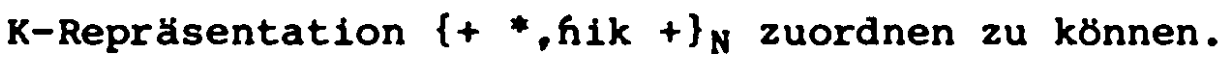

Für die Maskulina (136) gilt folgende AF-Regel:

$$
\begin{aligned}
\left(\text { AF 4.2.1) } \mathrm{K} \rightarrow\{+*, \text { hik }+\}_{\mathrm{N}} /\{+ \text { ova }+\}\right. & \mathrm{V} \\
& {\left[\begin{array}{l}
+ \text { FUNKTION } \\
+ \text { TRAGER } \\
+ \text { BELEBT } \\
+ \text { MENSCHLICH } \\
+ \text { TATIGKEIT } \\
+ \text { MASKULINUM }
\end{array}\right] }
\end{aligned}
$$

Zusammenfassend last sich zur Bildung der Maskulina (135) und (136) folgendes feststellen:

(135.1) (a) Die Maskulina (135) und (136) werden von imperfekti(136.1) ven Verben gebildet; die Maskulina (135) von den Verben auf -ac, die Maskulina (136) von den Verben auf -owac: 
(b) Die Maskulina (135) sind gegenüber den Maskulina (136) bezüglich [- BELEBT; + INSTRUMENTALITAT] markiert, desgleichen bezüglich der K-Repräsentation; die Maskulina (136) dagegen sind bezüglich der TM-Reprásentation und bezúglich der K-Repräsentation gegenüber den Maskulina (136) markiert;

(c) Gemeinsam haben (135) und (136) die Merkmale [+ TRAger] und [+ TÄTIGREIT];

(d) Für die phonologische Endableitung gelten u.a. (DL $29(1)$ ) und (PL26).

Sowohl von imperfektiven, perfektiven als auch iterativen Verbalstämmen werden folgende Maskulina gebildet:

(137) (1)

wychowawca 'Brzieher'
wykonawca 'Interpret'
znawaa 'Kennex'

(ii) przesladowca 'Verfolger' hodowca 'züchter' kierowca 'Chauffeur'

(1ii) sprzedawca 'Verkäufer' dawca 'Geber' rozsiewca 'Säer' spożywca 'Verzehrer' odkrywca 'Entdecker'

(1v) tworca 'schöpfer' przywodzca 'Anführer' radca 'Ratsherr' raqdea 'Regent' zastepca 'Vertreter'
- wychowac 'erziehen'

- wykonac 'ausführen'

- znac 'kennen'

- praesladowad 'verfolgen'

- hodowad 'züchten'

- kierowad 'lenken'

- sprzedawac 'verkaufen'

- dawad 'geben'

- rozsiewac 'aussäen'

- rozlewal 'ausgieben'

- spoziywad 'verzehren'

- odkrywad 'entdecken'

- tworaye 'schaffen'

- praywodzic 'anführen'

- radzic 'raten'

- raqdaic 'regieren'

- zastqpic 'vertreten'

Die vier Gruppen in (137) unterscheiden sich darin von einander, $\mathrm{daB}(137(i))$ von imperfektiven und perfektiven verben auf -ac, (137(1i)) von imperfektiven Verben auf -owac, (137(1i1)) von iterativen Verben auf -awac, -ewac, -ywac und $(137$ (iv)) von imperfektiven Verben auf $-i c$ bzw. $-y c$ gebildet werden. In $(137(1))$ wird dem perfektiven Verbalstamm die TF-Repräsentation $\{+a v+\}_{T F}$ hinzugefügt. Die Maskulina (137) werden wie Feminina - vgl. $\S$ 7.2 .2 - flektiert. ${ }^{80}$

Wie für (135) und (136) gelten auch hier die Paraphrasen: ten, co wychowuje, co wykonuje, co zna, co przerladuje, co sprzedaje, co rozsiewa, co tworzy,... 'Elner, der erzieht, der ausführt, der kennt, der verfolgt, der verkauft, der aussät, der 
schöpft'. Demnach gelten auch für (137) die Merkmale der 'Bestimmung' im Sinne von 'Tätigkeit', die einem 'belebten Träger' zugeordnet sind.

Die Maskulina (137) unterscheiden sich von den Maskulina (135) bezüglich der verbalen TM-Repräsentationen, auBer in (137(i)), wo allerdings beim imperfektiven verbalstamm ein TF hinzukomm: also sind die Maskulina (135) gegenüber (137) bezüglich der verbalen TM-Repräsentationen und im Falle (137(i)) bezüglich der 'Imperfektivität' des Verbalstammes auf -ac - ohne Hinzufügung einer TF-Repräsentation - markiert. Die Maskulina (136) dagegen sind gegenüber den Maskulina (137) - außer (137(ii)) - bezüglich des Verbalstammes -owat markiert.

Für die Maskulina (137) läBt sich folgende AF-Regel aufstellen: (AF 4.3 .1$)$

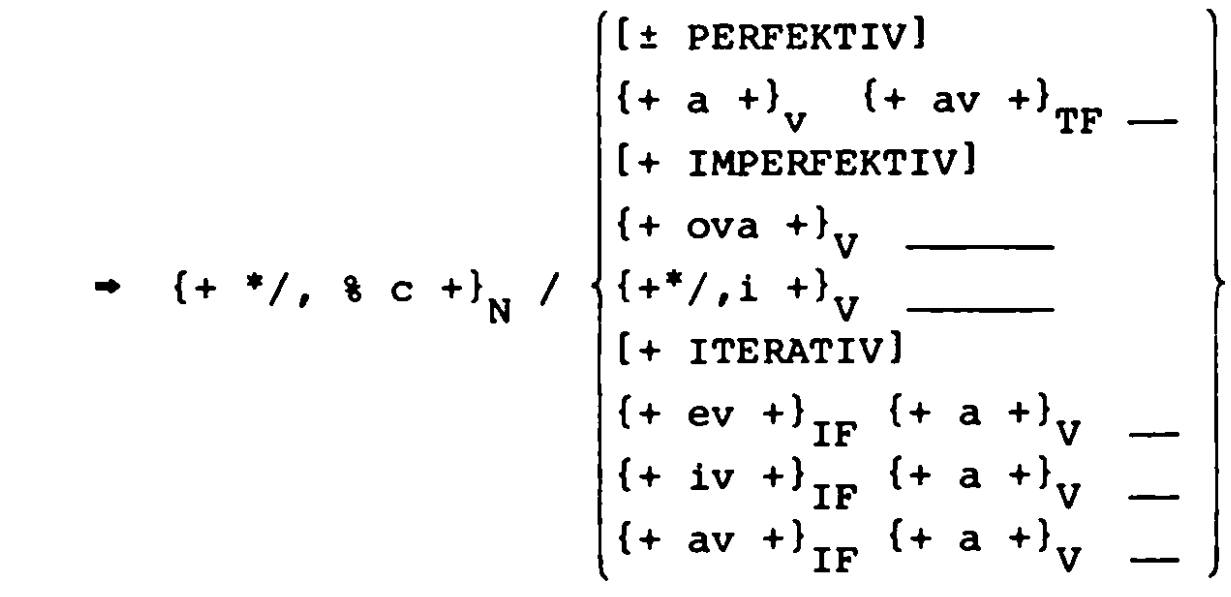

$$
\begin{aligned}
& {\left[\begin{array}{l}
+ \text { FUNKTION } \\
+ \text { TRAGER } \\
+ \text { BELEBT } \\
+ \text { MENSCHLICH } \\
+ \text { TÄTIGKEIT } \\
+ \text { MASKULINUM }
\end{array}\right]}
\end{aligned}
$$

Für die Bildung der Maskulina (137) läBt sich zusammenfassend folgendes feststellen:

(137.1) (a) Die Maskulina (137) werden von Perfektiva und Imperfektiva auf $-a c$, von Imperfektiva -ic und $-y c$, von Iterativa auf -ewac, -ywac, -awac, sowie - selten von Imperfektiva auf -owac gebildet;

(b) Gegenüber den Maskulina (135) und (136) sind die Maskulina (137) bezüglich der TM-Repräsentation 
$\{+* /, i+\}_{v}$ sowie bezüglich [+ ITERATIV] markiert;

(c) Für die phonologische Endableitung gelten u.a. (PL 16), (PL26) und (DL29(1)).

Aus der Gegenüberstellung von (135.1), (136.1) und (137.1) geht u.a. hervor, daß die unterschiedliche nominale K-Repräsentation nicht in erster Linie durch die inhärenten Merkmale bedingt ist, sondern durch die morphonemische Repräsentation des jeweiligen Verbalstammes, von dem das jeweilige Maskulinum gebildet wird.

Die Maskulina (135(i)), (136) und (137) haben folgende feminine Pendants :

$\begin{array}{ll}\text { pracowniczka 'Arbeiterin' } & \text { - pracownik } \\ \text { bojowniczka 'Kämpferin' } & \text { - bojownik } \\ \text { kierowniczka 'Leiterin' } & \text { - kierownik } \\ \text { wedrowniczka 'Wanderin' } & \text { - wedrownik } \\ \text { buntowniczka 'Rebellin' } & \text { - buntownik } \\ \text { spawaczka 'SchweiBerin' } & \text { - spawacz } \\ \text { badaczka 'Forscherin' } & \text { - badacz } \\ \text { tkaczka 'Weberin' } & \text { - tkacz } \\ \text { biegaczka 'Läuferin' } & \text { - biegacz } \\ \text { dziazaczka 'Aktivistin' } & \text { - dziatacz } \\ \text { wychowawczyni 'Erzieherin' } & \text { - wychowawca } \\ \text { wykonawczyni 'Interpretin' } & \text { - wykonawca } \\ \text { dawczyni 'Geberin' } & \text { - dawca } \\ \text { sprzedawczyni 'Verkäuferin' } & \text { - sprzedawca } \\ \text { zastepczyni 'Vertreterin' } & \text { - zastepca }\end{array}$

Ebenso wie bei den denominalen Feminina (113) und (119) sind die Maskulina (136), (135(i)) sowie (137) Voraussetzing für die Bildung der Feminina (138). Für $(138(i))$ und $(138(i i))$ gilt auch die gleiche $K$-Repräsentation, nämlich $\{+* 8 K+\}_{N}$, für $(138(i i i))$ dagegen gilt die $K$-Repräsentation $\left\{t^{*} i \hat{h}+\right\}_{N}$. Da es sich bei den Feminina (138) um keine deverbalen Nomina, sondern um denominale Nomina handelt, wird auf das Aufstellen der AF-Regeln verzichtet. Dabei wäre noch hinzuzufügen, daß die femininen Pendants auf die 'belebten' deverbalen Maskulina beschränkt sind.

$\mathrm{Zu}$ den deverbalen 'belebten' Maskulina $z$ ählen auch folgende:

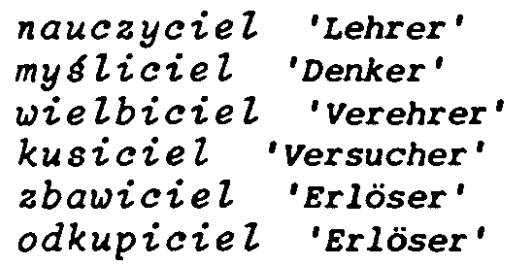

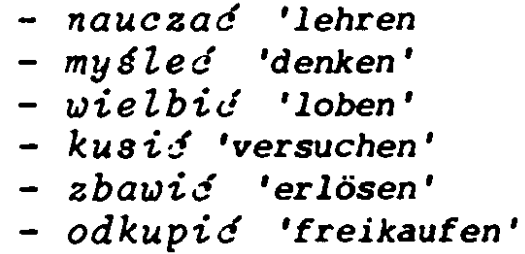

- nauczac 'lehren

- my\&lec 'denken'

- wielbici 'Ioben'

- zbawis 'erlösen'

- odkupice 'freikaufen' 
Für die Maskulina (139) gelten die gleichen inhärenten Merkmale wie für die Maskulina (135(i)), (136) und (137). Ihre K-Repräsentation ist jedoch $\left\{+* 1{ }^{*}{ }^{\prime} e{ }^{\prime}+\right\}_{N}$; diese $\mathrm{K}$-Repräsentation ist nicht mit dem Auslaut der wörter, wie z.B. przyjacię 'Freund' zu verwechseln, wo eine Vokalalternation - vgl. GEN PL przyjacióz - enthalten 1st. Die meisten dieser Maskulina werden nicht mehr gebraucht. Die noch aktuellen werden - in der Regel - von Imperfektiva mit unterschiedlicher verbaler TM-Repräsentation, auBer $\{+ \text { ova }+\}_{v}$ gebildet: die aus der sprache der kirche bzw. der Bibel stammenden unter ihnen, wie z.B. zbawiciel, odkupiciel, werden von Perfektiva gebildet.

Die Abgrenzung der Maskulina (139) von den Maskulina (136) ist einfach, da in (139) Verbalstämme auf -owat ausgeschlossen werden. Um jedoch (139) von (135(1)) und (137) abzugrenzen und damit die K-Repräsentation zu erklären, bedürfte es im Hinblick auf die Feststellung, daB sich (139) weder durch die inhărenten Merkmale noch durch die verbalen TM-Repräsentationen von den anderen unterschelden, eines zusätzlichen Merkmals, das den 'geistigen' Charakter des bezeichneten 'Tätigkeitstrăgers' kennzeichnen wïrde.

Da jedoch in Falle der Maskulina (139) die Tendenz zur Unproauktivitä - vgl. IAT - vorherrschend ist, wird auch hier auf das Aufstellen einer $A F-R e g e l$ verzichtet.

Es wăre noch hinzuzufügen, das die meisten, noch aktuellen Maskulina (139) ebenfalls feminine Pendants haben. Die Bildung der femininen Pendants setzt die Bildung der Maskulina (139) voraus, so z.B. In nauczyciel - nauczycielka 'Lehrer-Lehrerin' oder wielbiciel - wielbicielka 'Verehrer-Verehrerin'. Die femininen Pendants sind also gegenüber den Maskulina (139) bezüglich Genus und bezulglich der femininen $k$-Repräsentation $\{+* k+\}_{N}$ markiert. Demnach setzen sie die Reihe der 'belebten, menschlichen' Feminina (138) sowie (113) und (119) fort, die mit Hilfe der gleichen K-Reprăsentation von den jeweiligen Maskulina gebildet werden.

Die Reihe der denominalen Bezelchnungen für 'Räume' in (114) und in (115) wird durch weitere Feminina, die 'Räume' bezeichnen, doch deverbal sind, ergänzt: in diese Reihe gehören auch die deadjektivischen Feminina (133), da all diesen Femininadie 
$\mathrm{K}$-Repräsentation $\{+*, 8 \mathrm{~h}+\}_{\mathrm{N}}$ gemeinsam ist. $\mathrm{zu}$ den deverbalen Feminina, die 'Răume' bezeichnen, gehören u.v.a.:
(140) (1) jadalnia 'BBzimmer' pijalnia 'Trinkhalle'
- jadad 'essen' (ITBR)
sypialnia 'Schlafzimer'
poczekalnia 'warteraum'
- pijac 'trinken'(ITER)
- oypiad 'schlafen'(ITER)
(11) uczelnia 'Hochschule'
warzelnia 'Sudhaus'
pocrekac 'warten'(PERP)
warzelnia 'Sudhaus'
(1ii) jadzodajnia 'Speisehaus'
myjnia 'Autowaschstelle'
- ucayc 'lehren' (IPF)
- warayc 'brauen'(IPF)
myjnia 'Autowaschstelle'
- jadzo + dac'speisetgeben'
rysownia 'Zeichensaal' montownia 'Montagehalle'
(iv) pracownia 'Werkstatt'
(v) poradnia 'Beratungsstelle' praychodnia 'Poliklinik' chZodnia 'Kühlraum'
- myc 'waschen' (IPF)
(PERF)
- pracowal 'arbeiten'(IPF)
- ryeowad 'zeichnen'(IPF)
- montowad 'montieren'(IPF)
- poradzid 'beraten'(PBRF)
- praychodaiC 'komen'(IPF)
- chzodzic 'kühlen'(IPF)

In $(140(i))$ liegen den Feminina hauptsăchlich iterative verben zugrunde; sowohl bei den iterativen als auch bei den perfektiven und imperfektiven handelt es sich um verben auf -ac. Die zuordnung der nominalen $K-$ Repräsentation setzt die Annahme eines TF, nämlich $\{+a l+\}_{T F}$ voraus. In $(140(1 i))$ liegen den Feminina die Verben auf -yc zugrunde; die zuordnung der nominalen K-Reprăsentation setzt die Einbeziehung ebenfalls eines TF, nämlich $\{+ \text { el }+\}_{\text {TF }}$ voraus. $^{81}$ In $(140(111))$ ist es einmal das verb myc. das (134) entsprechend die $K$-Reprasentation $\{+\emptyset+\}$ vat, zum anderen ist es das Verb dac, ein Perfektivum, das zu den unregelmăbig flektierten Verben gehort. Wollte man im Falle des Femininum jadzodajnia vom Imperfektivum bzw. Iterativum dawac ausgehen, so mủbte das Nomen *jadzodawalnia lauten; dieses Nomen gibt es jedoch nicht im Lexikon, also muB die Bildung des Nomen von dac ausgehen, wobei ebenfalls ein TF, nămlich $\{+j+\}_{T F}$ einzusetzen ist.

Der Wortstamm in jadzodajnia entspricht der regulären Bildung der polnischen Komposita. Die polnischen Komposita können zusammengesetzt sein aus den stämmen: $N+N ; N+V ; N+A ; V+N i V+$ $+A ; A+N$ sowie $A+A ;$ nicht möglich sind im Polnischen die Zusammensetzungen $V+V$ sowie $A+V$. $Z$ wischen den so zusammengesetzten wortstämmen steht in der Regel ein INTERFIX $\{+0+\}$, das völlig unabhängig von der lexikalischen Kategorie, durch welche der jeweilige Wortstamn im Kompositum gekennzelchnet ist, die 
beiden stämme mit einander verbindet, so z.B. auch in drog-o-wskaz 'Wegweiser' oder in drog-o-cenny 'wertvoll'. ${ }^{82}$

In $(140(\mathrm{iv}))$ und in $(140(\mathrm{v}))$ wird kein TF eingesetzt. In beiden Gruppen wird der vokalische verbauslaut - entsprechend (DL29) getilgt.

Die Feminina (140) können folgendermaßen paraphrasiert werden: sala, w ktorej sie jada, w której siq pija, w ktorej sie sypia, $\omega$ ktorej sie uczy, w ktorej sif jadto daje, w ktorej sif pracu$j e$, do ktorej sie praychodzi 'saal, in dem man iBt, in dem man trinkt, in dem man schläf, in dem man lehrt, in dem man Essen ausgibt, in dem man arbeitet, in den man kommt'. Der 'Raum' ist also bezüglich des vom Verbalstamm Bezeichneten 'bestimmt'; da es sich um das 'Bestimmtsein' eines 'nicht belebten' 'Raumes' bezüglich einer 'Tätigkeit' handelt, gilt für alle Feminina in (140) das Merkmal [+ INSTRUMENTALITÄT]; es handelt sich also um einen 'nicht belebten' 'Funktionsträger'.

Für die Feminina (140) lăBt sich folgende AF-Regel aufstellen:

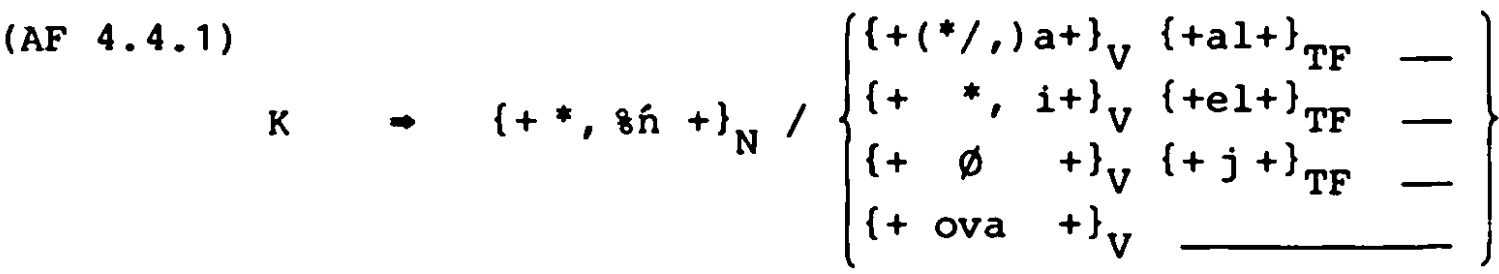

$$
\begin{aligned}
& {\left[\begin{array}{l}
+ \text { FUNKTION } \\
+ \text { TRÄGER } \\
- \text { BELEBT } \\
+ \text { RAUMLICH } \\
+ \text { BESTIMMTSEIN } \\
+ \text { INSTRUMENTALITAT } \\
+ \text { FEMININUM }
\end{array}\right]}
\end{aligned}
$$

Zusammenfassend läBt sich zur Bildung der Feminina (140) folgendes feststellen:

(140.1) (a) Die Feminina (140) werden von Verben gebildet; dabei scheint nicht so sehr der Aspekt bzw. die Aktionsart wie vielmehr die verbale TM-Reprăsentation die entscheidende Rolle zu spielen;

(b) Die Feminina (140) tragen - wie übrigens die Feminina 
(114), (115) und (133)-das Merkmal [+ RAUMIICH];

(c) Da es sich bei den Feminina (140) un 'nicht belebte' 'Tatigkeitstrăger' handelt, werden sie durch I+ INSTRUMENTALITAT] gekennzeichnet;

(d) Gegenüber den Feminina (114), (115) und (133) sind die Feminina (140) bezüglich 'deverbal' markiert;

(e) Für die phonologische Endableitung gelten u.a. (PL 11), (PL16), (PL26) und (DL29).

Ebenso durch das Merkmal [+ INSTRUMENTALITÁT] sind folgende deverbale Feminina gekennzeichnet:

$$
\begin{aligned}
& \text { rysownica 'Reibbrett' - rysowad 'zeichnen' } \\
& \text { spawalnica 'SchweiBgerat' - spawal 'schweiBen' } \\
& \text { walkownica 'Mudelwalker/-brett'- waZkowal 'walken' } \\
& \text { strielnica 'SchieBstand' - strzelad 'schieBen' }
\end{aligned}
$$

Doch im Gegensatz zu den Feminina (140) bezeichnen die Feminina (141) keine 'Răume' in 'dreidimensionalem' Sinne. Mit den Merkmalen [+ BESTIMMTSEIN; + INSTRUMENTALITAT] sOwie [- RAUMLICH] entsprechen diese Feminina den Feminina (120(1i)), wenngleich für (141) andere Paraphrasierungen gelten, nămlich: deska, na ktorej sif rysuje, na ktorej oif wazkuje 'Brett, auf dem man zeichnet, auf dem man walkt' bzw. prsyrsqd sa pomocq ktorego sig spawa 'Gerăt, mit dessen Hilfe man schwelBt' bzw. ursqdrenie, gdzie sif strsela 'Einrichtung, in der man schiest'.

Bezüglich [+ INSTRUMENTALITAKT] sowie [- RAUMIICH] stehen die Feminina (141) auch in Beziehung zu den deverbalen Maskulina (135 (1i1)). Um (141) von (135) im Hinblick auf die unterschiedliche Affiglerung abgrenzen zu können, mübten eventuell die beiden Gruppen zugrundeliegenden Wortverbindungen berucksichtigt werden, was jedoch nicht einfach sein sollte, da es sich hier un Wortverbindungen handelt, die in der Gegenwartssprache nicht mehr aktuell bzw. schwer rekonstrulerbar sind. Diese Wortverbindungen mübtennämlich ein Nomen enthalten, das AufschluB uber das Genus Femininum bzw. Maskulinum gibt; demnach mubte hier die diachronische sicht miteinbezogen werden. Wollte man die Abgrenzung ausschlieblich synchronisch bestlmmen, so wăren weitere Merkmale notwendig, die das Merkmal der 'Instrumentalitat' differenzieren wïden; hier ware aber elne gesonderte Untersuchung - desgleichen bei der diachronischen sicht - angebracht. AuBer 
den denominalen und den deverbalen Feminina, die durch die Merkmale [+ INSTRUMENTALITÄT; - RAUMLICH ] gekennzeichnet sind, gibt es nämlich noch die deadjektivischen Feminina mit dengleichen Merkmalen, die in $\S 8.3$ unberücksichtigt bleiben, nämlich: szachownica - deska szachowa 'Schachbrett' oder piaskownica - skrzynia piaskowa 'Sandkasten'. Im Hinblick auf den großen Umfang, den die Abgrenzungen erforderlich machen würden, beschränke ich mich hier auf die Feststellung, daß die Feminina (141) gegenüber den Maskulina (135(iii)) bezüglich des Genus markiert sind; auf das Aufstellen einer AF-Regel wird ebenfalls verzichtet.

Zusammenfassend läßt sich zur Bildung der Feminina (141) folgendes feststellen:

(141.1)(a) Die Feminina (141) werden von Verben gebildet, wobei die Perfektiva ausgeschlossen sind;

(b) Die Feminina (141) bezeichnen 'Instrumentalitat' neben dem Merkmal [- RAUMLICH]; damit stehen sie in Opposition $z u$ den Feminina (140), die das Merkmal [+ RÄUMLICH] haben;

(c) Die Feminina (141) haben die gleichen Merkmale wie die denominalen Feminina (120) sowie die deadjektivischen Feminina vom Typ szachownica;

(d) Die Feminina haben die K-Repräsentation $\left\{+*, f_{i c}\right\}_{N}$;

(e) Für die phonologische Endableitung gelten u.a. (PL11) bzw. (PL26).

\subsubsection{DIE SOGENANNTEN VERBALWORTER}

Die sog. Verbalwörter werden hier zu den deverbalen Nomina gezählt, weil sie u.a. den Kategorien des Genus, des Numerus, des Kasus sowie den inhärenten Merkmalen (92) des Nomen unterliegen. Im Gegensatz zu den deverbalen Nomina in $\S 8.4$ sind die sog. Verbalwörter durch das Merkmal [- TRAGER] gekennzeichnet, d.h. sie bezeichnen die 'Funktion' selbst, ohnen deren 'Träger'; so bezeichnet z.B. das Verbalwort bieg 'Lauf' die 'Funktion' für die 'Bestimmung' im sinne der 'Tătigkeit' biegae 'laufen', während der 'Träger' dieser 'Tätigkeit' - vgl. (135(i)) - biegacz 'Läufer' ist. Da diese Eigenschaft allen deverbalen Nomina, deren Bildung in diesem Kapitel erörtert werden soll, gemeinsam ist, 
werden für alle aufzustellenden AF-Regeln die Merkmale (142) impliziert.

$$
\left[\begin{array}{l}
+ \text { FUNKTION } \\
- \text { TRÄGER } \\
+ \text { BESTIMMUNG } \\
+ \text { INDIVIDUATIVUM }
\end{array}\right]
$$

Das Merkmal [+ INDIVIDUATIVUM] in (142) entspricht der 'zählbarkeit der hier in Frage kommenden Nomina.

Von einer regelmäßigen zuordnung der nominalen K-Repräsentation kann hier ebensowenig die Rede sein, wie bei den deverbalen Agentiva in $\S 8.4$. Im allgemeinen herrscht im Polnischen die Tendenz vor, die verbale Wurzel-Repräsentation mit der nominalen $K-R e-$ präsentation $\{+\varnothing+\}_{N}$ zu versehen, um das 'Resultat' der vom Verb bezeichneten 'Tätigkeit' zu bezeichnen, so z.B. krzyk - krzyczed 'Schrei - schreien' oder praca - pracowad 'Arbeit - arbeiten'. Demnach wird diese nominale K-Repräsentation sowohl dem Maskulinum als auch dem Femininum zugeordnet. Es kommt aber auch vor, daß diese Möglichkeit der Nominalisierung nicht gegeben ist, z.B. dann, wenn dieses Nomen im Lexikon nicht vorhanden ist; dies in Falle von *osiqg - osiqgae 'erreichen'. Dafür ist aber osiqgnifcie - osiqgnqf 'Erreichtes, Errungenschaft - (momentan, einmalig) erreichen' möglich, d.i. ein Nomen mit dem Genus Neutrum vom semelfaktiven Verb gebildet.

Bei der Bildung der deverbalen Nomina mit der Bedeutung 'Resultat' kommen unterschiedliche Auslautrepräsentationen hinzu, so z.B. pzacz - pzaḱac 'Weinen - weinen' aber skok - skoczyc 'Sprung - springen', pomysz - pomyśzed 'Einfall, Idee - nachdenken' aber my $\underline{l} \underline{l}$ - my $\not \underline{l} e c$ 'Gedanke - denken'. Die Beispiele skok und pomys $z$ lassen annehmen, daß mit der zuordnung der nominalen $K$-Repraisentation $\{+\varnothing+\}_{N}$ nicht nur der Vokal der verbalen TM-Repräsentation getilgt wird, sondern auch der Konsonantenwechsel $\{*\}$, und daß diese Tilgung mit der zurodnung des Genus Maskulinum verbunden ist; während der konsonantenwechsel $\{,\} \mathrm{mit}$ der zuordnung des Genus Femininum verbunden $2 u$ sein scheint, so im Beispiel my\&l. Doch sowohl das Femininum my $\xi l$ als auch das Maskulinum płacz scheinen zu den Ausnahmen zu gehören; denn im allgemeinen herrscht im Polnischen die Tendenz vor, den Auslautwechsel der verbalen wurzel nicht mit dem Nomen, sondern mit 
dem IMPERATIV zu assozileren, so z.B. - vgl. dazu Baudouin de Courtenay $(1908,170)$ - in stan / stan, twor / twora, chod / / chodz' 'Stand/bleib stehen, Gebilde/schaffe, Gang/gehe, komme'.

Im allgemeinen kann davon ausgegangen werden, daB die Maskulina wie krayk, ryk, kroj, przekroj 'Schrei, Gebrüll, Schnitt, Durchschnitt' sowie die Feminina wie wiara, praca, para 'Glaube, Arbeit, Dampf' das 'Resultat', die Neutra wie krzyczenie, ryczenie, krojenie, przekrojenie, wierzenie, pracowanie, parowanie 'schreien, Brüllen, Schneiden, Durchschneiden, Glauben, Arbeiten, Danpfen' dagegen den 'Verlauf' der jeweiligen 'Tatigkeit' bezeichnen. Wenn jemand im Polnischen sagt:

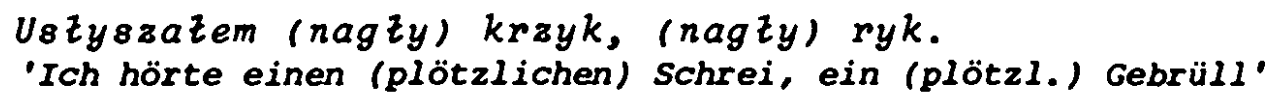

so heibt das, daB ein unbestimmtes wesen geschrien bzw. gebrüllt hat, daB krzyk bzw. ryk das 'Resultat' der 'Tätigkeit' dieses unbestimmten Wesens, das selbstverstăndlich auch năher bezeichnet werden kann, 1st, die durch krayczed bzw. ryczed bezeichnet wird. ${ }^{83}$ Doch wenn jemand sagt:

(144) Ryczenie trwazo caza noc.

'Das Brüllen dauerte die ganze Nacht'

so heibt das, daB statt des 'Resultats' des 'Brüllens' sein'Verlauf' zeitlich năher bestimmt wird, nämlich durch cazq noc 'die ganze Nacht hindurch', da das Nomen ryczenie - vom Durativ gebildet - selbst keinen năheren Hinweis auf die 'Dauer' des 'Verlaufs' enthält, sondern allein auf den nicht năher bestimmten 'Verlauf' der 'Tatigkeit' ryczec hinweist; das gleiche gilt für krzyczenie vs. krzyczec.

Aber wie ist dann das Nomen ryknifcie '(etwa) Aufbrüllen' einzustufen? Dieses Nomen wird nicht vom imperfektiven ryczed, sondern vom perfektiv-momentanen ryknqc ' (etwa) einmal aufbrüllen' gebildet, das entsprechend (88) das Merkmal [+ SEMELFAKTIV] trăgt. Es handelt sich hier also - vgl. Isačenko $(1975, \S 217 . B . I$.$) - um$ ein Momentanverb. Dieses Momentanverb hat zur Folge, daB das Nomen rykniecie ebenfalls als ein 'Resultat' einzustufen ist; dafür spricht u.a. auch die Feststellung, daB *krayknigcie im DOR weder gebucht noch belegt ist und daB die 'momentan-einmalige' Kennzeichnung der 'Tätigkeit' krayknqc 'aufschreien' ebenfalls von krayk getragen wird. Geht man jedoch davon aus, daB ryk und 
krzyk nicht vom perfektiv-momentanen, sondern vom impferfektiv-durativen verb gebildet - und dies ist in anderen Beispielen überwlegend der Fall - werden, so ist ryknifcie, da von ryknqc gebildet, zwar als ein 'Resultat' einzustufen, jedoch als das 'Resultat' einer 'Tátigke1t', deren 'Verlauf' - im Gegensatz zum durativen ryczec - durch das 'Momentane' bzw. 'Einmalige' näher bezeichnet wird. Also gilt für ryknifeie nicht nur das 'Resultat' einer 'Tätigke1t', sondern auch deren 'momentaner' bzw. 'nur einmal ausgeführter Verlauf'. Wird das Merkmal [ \pm EINMALIG] hinzugezogen, so gilt [+ EINMALIG] für ryknifcie, [-EINMALIG] dagegen für ryczenie. Für ryk braucht keine năhere kennzeichnung der 'Tätigkeit' angenommen $z u$ werden, da deren 'Verlauf' negativ markiert ist; dafür gilt [+ RESULTAT] aber auch nur für ryk, nicht für die anderen Nomina, die durch [+ VERIAUf] gekennzeichnet sind. Im Falle von ryknigcie impliziert also das Merkmal [+ EINMALIG] das Merkmal [+ RESULTAT], das gegen [+ VERLAUF] abgegrenzt ist. Demnach wird die dem Nomen zugrundeliegende 'Tatigkeit' entweder durch [+ VERLAUF] oder durch [ - VERLAUF; + RESULTAT] gekennzeichnet, wăhrend [ \pm EINMALIG] allein das Merkmal [+ VERLAUF] betrifft.

Das Merkmal [- EINMALIG] gilt folglich auch für das Nomen porykiwanie ' (etwa) Ab-Und-Zu-Brullen'; dieses Nomen wird vom iterativen porykiwad gebildet und weist nicht nur auf den 'Verlauf' der 'Tätigkeit', sondern zusätzlich auf deren 'Wiederholung' hin. Dafür gilt neben [+ VERLAUF] das Merkmal [+ WIEDERHOLT]. Fủr die jeweilige Nominalisierung des Verbs gelten die Merkmale (145), die der weiteren Beschreibung der sog. Verbalworter zugrunde gelegt werden.

In (145) schlieben sich nicht nur [+ VERLAUF] und [+ RESULTAT], sondern auch [+ WIEDERHOLT] und [+ MOMENTAN] gegenseitig aus. Die Merkmale (145) gelten als Ergănzungen der Merkmale [+ $\mathrm{ZU}-$ STAND] sowie [+ TÄTIGKEIT] in (94).

AuBer den momentanen wurden bisher noch keine perfektiven Verben berücksichtigt, die nominalisiert werden. Im Falle von z.B. zabicie 'Totung, Totschlag'; pobicie 'MiBhandlung, sieg'; wybicie 'Herausschlagen' handelt es sich um Nominalisierungen der Perfektiva zabic, pobic, wybic 'totschlagen, schlagen/besiegen, ausschlagen'. In allen drel fällen kann davon ausgegangen werden, 
daB es sich um das 'Resultat' der durch [- DELIMITATIV] gekennzeichneten zeitlich abgeschlossenen 'Tätigkeit' handelt; dennes heibt z.B. zabicie kota 'Tötung der Katze'; pobicie drużyny pizkarskiej 'Schlagen der FuBballmannschaft'; wybicie szyby okiennej 'Herausschlagen einer Fensterscheibe'; wörtlich müBte es im Deutschen heiBen: 'das Getötet-Haben; das Geschlagen-Haben; das Herausgeschlagen-Haben' . Der 'Verlauf' kann entweder vom nichtprăfigierten, imperfektiv-durativen bić, nämlich bicie 'Schlagen', oder vom imperfektiv-iterativen zabijac' (etwa) zu töten pflegen, wiederholt töten' gebildet werden, desgleichen von pobijac, wybijac ' (etwa) ab und $z u$, wiederholt miBhandeln, schlagen, besiegen/ ausschlagen, herausschlagen', nämlich: zabijanie, pobijanie, wybijanie.

(145) (i)

$$
\left[\begin{array}{l}
- \text { VERLAUF } \\
+ \text { RESULTAT }
\end{array}\right] \quad(r y k)
$$

$$
\left[\begin{array}{l}
+ \text { VERLAUF } \\
\text { - WIEDERHOLT } \\
\text { - EINMALIG }
\end{array}\right] \text { (ryczenie) }
$$

$$
\left[\begin{array}{l}
+ \text { VERLAUF } \\
- \text { WIEDERHOLT } \\
+ \text { EINMALIG }
\end{array}\right] \quad \text { (rykniecie) }
$$

(iv)

$$
\left[\begin{array}{l}
+ \text { VERLAUF } \\
+ \text { WIEDERHOLT }
\end{array}\right]
$$

(porykiwanie)

Für das 'Resultat' der perfektiven Verben zabic, pobic, wybic gelten die Merkmale (145(i)); für den 'Verlauf' der imperfektiv-iterativen Verben zabijac, pobijac, wybijac gelten die Merkmale (145(iv)), während $(145(i i))$ und (145(1ii)) für diese verben "unbesetzt" bleiben; dies schliebt jedoch nicht aus, daB kontextuell zabicie statt (145(iii)) als 'einmaliger Verlauf' und zabijanie anstelle $(145$ (ii)) als weder 'wiederholter' noch 'einmaliger Verlauf' eingesetzt werden können. Diese Feststellungen beschränken sich auf die Perfektiva mit der verbalen $\mathrm{K}-$ -Repräsentation - vgl. (134) - $\{+\emptyset+\}$.

Die anderen Perfektiva "verhalten" sich unterschiedlich. So wird z. B bei den Verben auf -af das 'Resultat' sowohl vom Perfektivum, wie z.B. odczyt - odczytac 'Vortrag-ablesen', als auch - und dies scheint überwiegend der Fall zu sein - vom iterati- 
wen Imperfektivum, wie z.B. odlew - odlewac 'AbguB-abgieBen', gebildet, der 'Verlauf' wird vom Perfektivum, nämlich odczytanie - odczytad 'Ablesen-ablesen', odlanie - odlac 'Abgieben-abgieben', der 'wiederholte Verlauf' dagegen vom Iterativum, nämlich odczytywanie - odczytywad 'Abzulesen-Pflegen', odlewanie - odlewad 'Ab-Und-Zu-AbgieBen', gebildet. Doch diese Nominalisierungen sollen eingehender anhand mehrerer Beispiele gezeigt werden.

(146) (i)

$$
\begin{aligned}
& \text { bzysk 'Aufblitzen' } \\
& \text { zysk 'Gewinn' } \\
& \text { spiew 'Gesang' } \\
& \text { wpzyw 'EinfluB' } \\
& \text { dopzyw 'ZufluB' } \\
& \text { odlew 'AbguB' }
\end{aligned}
$$

(ii) dar 'Gabe'

skrot 'Abkürzung'

powr $6 t$ 'Rückkehr'

zakret 'Kurve'

chwyt 'Griff'

donos "Denunziat"

(iii) pomysz 'Gedanke'

kaszel 'Husten'

jek 'Gestöhne'

opor 'Widerstand'

(iv) ksztazt 'Gestalt' zart 'scherz'
- bzyskad 'blitzen'(IPF)

- zyskas 'gewinnen'(IPF)

- Spiewae 'singen'(IPF)

- wpZywad 'einflieBen'(IPF-ITER)

- dopzywae 'zuflieBen'(IPF-ITER)

- odlewae 'abgieBen' (IPF-ITER)

- darzyd 'beschenken'(IPF)

- skrocic 'abkürzen'(PERF)

- powrocic 'zurückkehren'(PERF)

- zakrecic 'zudrehen' (PERF)

- chwycid 'fassen'(PERF)

- donosid 'denunzieren'(IPF)

- pomyslec 'nachdenken' (PERF)

- kaszled 'husten'(IPF)

- jeczyd 'stöhnen'(IPF)

- opieral sif 's. widersetzen'(IPF)

- ksataztowad 'gestalten'(IPP)

- zartowal 'scherzen'(IPF)

In (146) liegen ausschlieBlich Maskulina vor, die bei der Bezeichnung des 'Resultats' einer'Tätigkeit' den überwiegenden Anteil haben. Es kommen die Feminina (147) sowie die Neutra (148) hinzu.

7) (i) uchwaza 'BeschluB'
zabawa 'Amüsement'
(ii) cena 'Preis'
mowa 'Rede'
zdrada 'Verrat'
posada 'Stellung'
(iii) proßba 'Bitte'
wroiba 'Wahrsagung'
grozba 'Drohung'
ko\&ba 'Mahd'
szuzba 'Dienst'
(iv) mysl 'Gedanke'
korzy\&C 'Nutzen'
(v) para 'Dampf'
praca 'Arbeit'
(vi) choroba 'Krankheit'
zazoba 'Trauer'

- uchwalas 'beschließen'(IPF-ITER)

- zabawiad 'unterhalten' (IPF-ITER)

- cenić 'schätzen'(IPF)

- mowic 'reden,sprechen'(IPF)

- zdradzic 'verraten'(PERF)

- posadzid 'setzen'(PERF)

- prosid 'bitten'(IPF)

- wrozyd 'wahrsagen'(IPF)

- grozic 'drohen'(IPF)

- koside 'mähen'(IPF)

- szuzyc 'dienen'(IPF)

- my\&led 'denken'(IPF)

- korzystae 'Mutzen ziehen'(IPF)

- parowad 'dampfen'(IPF)

- pracowal arbeiten'(IPF)

- chorowad 'krank sein'(IPF)

- zalowad 'u.a. bereuen'(IPF) 
Zu den Neutra gehören u.v.a:

$$
\begin{array}{ll}
\text { zabicie 'Totschlag' } & \text { zabic 'totschlagen'(PERF) } \\
\text { ukzucie 'Stich' } & \text { - ukzuc 'stechen'(PERF) } \\
\text { przezycie 'Erlebnis' } & \text { - przezyd 'erleben'(PERF) } \\
\text { przeklecie 'Fluch' } & \text { - praeklqc 'verfluchen'(PERF) } \\
\text { przecigcie 'Schnitt' } & \text { - przeciqd 'durchschneiden'(PERF) }
\end{array}
$$

Die Nomina (146), (147) und (148) sind nach den verbalen $K-R e-$ prăsentationen (134) geordnet. Bel den Maskulina (146) und bei den Feminina (147) handelt es sich um Nomina, die von den verben auf $-a c,-i c /-y c,-e c$ und -owac gebildet werden. Beide Gruppen haben die nominale $K$-Repräsentation $\{+\varnothing+\}_{N}$, auBer den Feminina $(147(i 11))$ mit der $K$-Repräsentation $\{+*, 8 b+\}_{N}, v g l$. szuz-yc - szuz-b-a, den Feminina $(147$ (iv)) mit der K-Repräsentation $\{+,+\}_{N}, v g l$. korzyst-ac - korzysd, und den Feminina (147(vi)) mit der K-Repräsentation $\{+o b+\}_{N}$, vgl. $\dot{z} a z-o w a-c-\dot{z} a z-o b-a$. Die Merkmale des Aspekts bzw. der Aktionsart scheinen bei der Bildung der Maskulina (146) und bei der Bildung der Feminina (147) keine entscheidende Rolle zu spielen, da die gleiche K-Repräsentation sowohl den perfektiven als auch den imperfektiven bzw. iterativen Verbalstämmen zugeordnet wird. Die Berücksichtigung des Aspekts ist also hauptsächlich wegen der Neutra (148) notwendig, da diese auf den perfektiven Aspekt beschränkt sind. Demnach sind die Neutra (148) unter den deverbalen Nomina, die das 'Resultat' bezeichnen, gegenüber den Maskulina und den Feminina bezüglich [+ PERFEKTIV] markiert.

Die Feminina vom Typ szużba gehören zu den Relikten früherer Stadien der Geschichte der polnischen Sprache ${ }^{84}$ und gehören nicht mehr zu den produktiven Wortbildungstypen. Die Anzahl der wörter vom Typ zazoba ist auf 4 bis 5 begrenzt; die Anzahl der Wörter vom Typ korzyßC ist nicht erheblich gröBer. Daher werden diese drei Worttypen aus der aufustellenden AF-Regel ausgeschlossen.

Als Abgrenzung der Feminina (147) von den Maskulina (146) kann also nur die Feststellung gelten, daB die Feminina bezüglich Genus, das jedoch innerhalb der wortbildung keine morphonemische Repräsentation aufzuweisen hat, die es vom Genus Maskulinum abgrenzen wïrde, gegenüber den Maskulina (146) markiert sind. Die einzige Möglichkeit, die Feminina aufgrund einer morphonemischen Reprăsentation von den Maskulina abzugrenzen, wäre die Einbezlehung des NOM-SG-Flektivs; diese widerspräche jedoch der Tren- 
nung zwischen Wortbildung und Wortbeugung. Und selbst dann, wenn das feminine NOM-SG-Flektiv mit einbezogen würde, wäre nicht die Frage gelöst, nämlich: Welcher Verbalstamm wird dem Maskulinum und welcher dem Fimininum zugeordnet, wenn es um die Bildung des Nomen mit dem Merkmal des 'Resultats' geht.

In der aufzustellenden AF-Regel wird daher zwischen dem Genus Maskulinum und dem Genus Femininum nicht unterschieden. Diese Regel gilt ausschließlich für die produktiven Wortbildungstypen und betrifft demnach die Maskulina (146) sowie die Feminina $(147(i))$ und $(147(i i)) .85$

(AF 4.5.1)

[ \pm IMPERFEKTIV]

$$
\begin{aligned}
& K \quad \rightarrow \quad\{+\varnothing+\}_{N} /\left\{\begin{array}{l}
\{+(*,) a+\} v \\
\{+*, i+\} v \\
\{+*, e+\} v- \\
\{+o v a+\} v-
\end{array}\right\} \\
& {\left[\begin{array}{c}
- \text { BELEBT } \\
\pm \text { ZUSTAND } \\
- \text { VERLAUF } \\
+ \text { RESULTAT } \\
\left\{\begin{array}{l}
{[+ \text { MASKULINUM }]} \\
{[+ \text { FEMININUM }]}
\end{array}\right\}
\end{array}\right]}
\end{aligned}
$$

Das Merkmal [ \pm ZUSTAND] in (AF 4.5.1) entspricht entweder [+ ZUSTAND] oder [+ TÁTIGKEIT]. Als Beispiel für [+ ZUSTAND] gilt 2.B. odmiana 'Veränderung'. Das Merkmal [ \pm IMPERFEKTIV] betrifft alle in Frage kommenden Verbalstämme. (AF 4.5.1) impliziert die Merkmale (142).

Für die Bestimmung der nominalen $\mathrm{K}$-Repräsentation der Neutra (148) gibt es zwei Möglichkeiten: zum einen könnte man davon ausgehen, daß z.B. przeklqc bereits die vollständige K-Repräsentation von przekleci-e zugrunde liegt, wenngleich man dann keinen verbalen Klassifikator $\{+\tilde{o}+\} v$, sondern einen Verbalklassifikator mit der Alternation der Nasalvokale annehmen müBte, zum anderen bietet sich die Möglichkeit, eine nominale K-Repräsentation $\{+\tilde{e} c \hat{c}+\}_{N}$ anzunehmen und die Affigierung dieser Nomina von der Affigierung der anderen zu trennen. Der Einfachheit wegen wird für die aufzustellenden AF-Regeln die letztgenannte Möglichkeit angewandt, $d . h .$, daB die Affigierung der Nomina vom Typ 
przecifcie die Affigierung der anderen Neutra ausschliebt.

Fur die Neutra, die von den Verben auf -qC gebildet werden, gilt also folgende AF-Regel:

(AF 4.5.2)

$$
\begin{aligned}
& {\left[\begin{array}{l}
\text { - IMPERFEKTIV } \\
- \text { DELIMITATIV }
\end{array}\right]} \\
& \mathrm{K} \rightarrow\{+\tilde{e} \varepsilon+\}_{\mathrm{N}} /\{+\tilde{o}+\}_{\mathrm{V}} \\
& {\left[\begin{array}{l}
- \text { BELEBT } \\
\pm \text { ZUSTAND } \\
- \text { VERLAUF } \\
+ \text { RESULTAT } \\
+ \text { NEUTRUM }
\end{array}\right]}
\end{aligned}
$$

Für alle anderen Neutra (148) dagegen gilt folgende AF-Regel:

(AF 4.5 .3 )

$$
\mathrm{K} \rightarrow\{+\varepsilon+\}_{\mathrm{N}} /\{+\emptyset+\}_{\mathrm{V}}
$$

[WIE (AF 4.5.2)]

Zusammenfassend läst sich zur Bildung der deverbalen Nomina (146), (147) und (148) folgendes feststellen:

(146.1)(a)Die Maskulina (146), die Feminina (147) sowie die Neu(147.1) tra (148) werden von Verbalstämen gebildet und haben (148.1) das Merkmal [+ RESULTAT] gemeinsam;

(b) Gemeinsam haben auch diese Nomina die Merkmale [- TRXGER]; damit sind sie gegenüber den deverbalen Nomina in $\S 8.4$ bezüglich dieses Merkmals markiert;

(c) Die Nomina mit dem Merkmal [+ REsulTAT] werden in der Regel durch Hinzufügung der nominalen $K$-Repräsentation $\{+\emptyset+\}_{N}$ bei gleichzeitiger Tilgung der verbalen TM-Reprasentation gebildet;

(d)Die Berücksichtigung des Aspekts ist für die Bildung der Neutra (148) notwendig; daher sind die Neutra (148) gegenüber den Maskulina (146) und den Feminina (147) bezüglich [ - IMPERFEKTIV; - DELIMITATIV] markiert;

(e)Die Feminina (147) sind gegenüber den Maskulina (146) allein bezulglich des Genus FEMININUM markiert;

(f)Flir die phonologische Endableitung gilt u.a. (PL29(1)). 
Die Neutra (148) haben auch imperfektive Gegenstücke, die sich darin unterscheiden, daß sie von nicht präfigierten verbalstämmen mit der TM-Repräsentation $\{+\emptyset+\} v$ gebildet werden. $\mathrm{zu}$ diesen Neutra gehören u.v.a.:

bicie 'Schlagen'
ciecie 'Schneiden'
kzucie 'Stechen'
zycie 'Leben'
mycie 'Waschen'
picie 'Trinken'
wycie 'Heulen'
szycie 'Nähen'

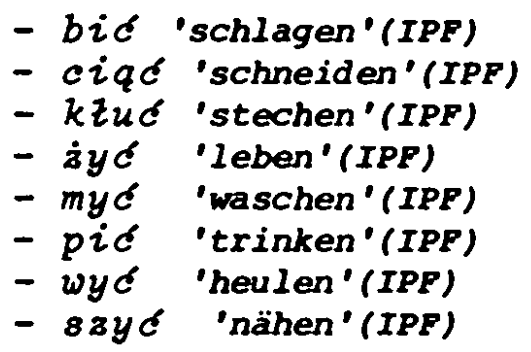

Sieht man davon ab, dab die Neutra (149) vereinzelt auch mit der Bedeutung 'Resultat' - z.B. ciecie '(Film-)Schnitt' - verwendet werden konnen, so kann diese Gruppe entsprechend (145(11)) gekennzeichnet werden.

Für die Neutra (149) läBt sich folgende AF-Regel aufstellen:

$(A F$ 4.6.1)

$$
\begin{aligned}
& {\left[\begin{array}{l}
+ \text { IMPERFERTIV } \\
- \text { ITERATIV }
\end{array}\right]} \\
& \mathrm{K} \quad \rightarrow \quad\{+\varepsilon+\}_{\mathrm{N}} /\{+\varnothing+\}_{\mathrm{V}} \\
& {\left[\begin{array}{l}
- \text { BELEBT } \\
\pm \text { ZUSTAND } \\
+ \text { VERLAUF } \\
- \text { WIEDERHOLT } \\
- \text { EINMALIG } \\
+ \text { NEUTRUM }
\end{array}\right]}
\end{aligned}
$$

Die Neutra (149) sind gegenüber den Neutra (148) bezüglich des Aspektmerkmals [+ IMPERFEKTIV] sowie bezüglich [+ VERLAUF] markiert.

Bezüglich [+ VERLAUF] sind auch die deverbalen Neutra (150) gegenüber den Neutra (148) markiert, wenngleich hier noch andere TM-Repräsentationen sowie andere K-Repräsentationen hinzukommen. Die deverbalen Neutra (150) sind einerseits nach ihren verbalen TM-Repräsentationen, anderseits nach dem imperfektiven und perfektiven Aspekt des Verbalstammes geordnet. Die TM-Repräsentationen entsprechen den Verben auf -ac, -owac, -ic/-yc und -ec, deren morphonemische Notation (134) entspricht. Die nominalen K-Repräsentationen sind einerselts $\{+a \hat{f}+\}_{N}$ für die Verbalstămme auf $-a c$ und -owac, anderseits $\{+ \text {,eh }+\}_{N}$ für die verbalstäm- 
me auf $-i c /-y c$ und $-e c$.

\begin{tabular}{|c|c|}
\hline $\begin{array}{l}\text { 150)(i)pisanie 'Schreiben' } \\
\text { czytanie 'Lesen' } \\
\text { spiewanie 'Singen' }\end{array}$ & $\begin{array}{l}\text { - pisad 'schreiben'(IPF) } \\
\text { - czytad 'lesen'(IPF) } \\
\text { - spiewad 'singen'(IPF) }\end{array}$ \\
\hline $\begin{array}{l}\text { (ii)odpisanie 'Abschreiben' } \\
\text { przeczytanie 'Durchlesen' } \\
\text { odspiewanie 'Absingen' }\end{array}$ & $\begin{array}{l}\text { - odpisad 'abschreiben'(PERF) } \\
\text { - przeczytac' 'durchlesen'(PERF) } \\
\text { - odspiewad 'absingen'(PERF) }\end{array}$ \\
\hline $\begin{array}{c}\text { (iii)chorowanie 'Kranksein' } \\
\text { czarowanie 'Zaubern' }\end{array}$ & $\begin{array}{l}\text { - chorowad 'krank sein'(IPF) } \\
\text { - czarowad 'zaubern'(IPF) }\end{array}$ \\
\hline $\begin{array}{c}\text { (iv)zachorowanie 'Erkrankung' } \\
\text { oczarowanie 'Entzücken' }\end{array}$ & $\begin{array}{l}\text { - zachorowad 'erkranken'(PERF) } \\
\text { - oczarowad 'bezaubern'(PERF) }\end{array}$ \\
\hline $\begin{array}{l}\text { (v)wqtpienie 'Zweifeln' } \\
\text { szuzienie 'Dienen' } \\
\text { koszenie 'Mähen' }\end{array}$ & $\begin{array}{l}\text { - wqtpid 'zweifeln'(IPF) } \\
\text { - szuzyc 'dienen'(IPF) } \\
\text { - kosic 'mähen'(IPF) }\end{array}$ \\
\hline $\begin{array}{l}\text { (vi)zwqtpienie 'Zweifel' } \\
\text { obszuzenie 'Bedienen' } \\
\text { dokoszenie 'Ausmähen' }\end{array}$ & $\begin{array}{l}\text { - zwqtpic 'bezweifeln'(PERF) } \\
\text { - obszusye 'bedienen'(PERF) } \\
\text { - dokosic'zu Ende mähen'(PERF) }\end{array}$ \\
\hline $\begin{array}{l}\text { (vii)myslenie 'Denken' } \\
\text { kleczenie 'Knien' } \\
\text { beczenie 'Heulen' }\end{array}$ & $\begin{array}{l}\text { - mysled 'denken'(IPF) } \\
\text { - kleczed 'knien'(IPF) } \\
\text { - beczed 'heulen'(IPF) }\end{array}$ \\
\hline $\begin{array}{l}\text { (viii) przemyslenie 'Durchdenken' } \\
\text { odkleczenie '(Strafe)Abknien' } \\
\text { rozbeczenie 'Losheulen' }\end{array}$ & $\begin{array}{l}\text { - przemysled 'durchdenken'(PERF) } \\
\text { - odkleczed 'abknien'(PERF) } \\
\text { - rozbeczed 'Losheulen'(PERF) }\end{array}$ \\
\hline
\end{tabular}

Generell kann davon ausgegangen werden, daB die Neutra (150) gegenüber den Neutra, die das 'Resultat' bezeichnen, bezüglich der Merkmale (145(ii)), also bezüglich 'Verlauf' markiert sind. Bei den von perfektiven Verbalstämmen gebildeten Neutra kann es jedoch der Fall sein, daB sle sowohl den 'Verlauf' als auch das 'Resultat' der 'Tätigkeit' bzw. des 'Zustands' bezeichnen. Dieser Fall tritt dann ein, wenn

(a) den Merkmalen (145(i)) keine lexikalische Repräsentation vom Typ (146) und (147) entspricht und/oder

(b) dem perfektiven Verbalstamm die resultative Aktionsart zugrunde liegt.

Fall (a) liegt z.B. in zwatpienie 'Zweifel' vor, Fall (b) z.B. in dokoszenie 'Ausmähen, Ausmahd'. Doch ebenso wie diese Neutra, die neben den Merkmalen (145(ii)) auch die Merkmale (145(i)) haben können, tragen auch einige Feminina in (147) neben den Merkmalen (145(i)) auch die Merkmale (145(ii)); so 2.B. praca 'Arbeit', in erster Linie das 'Resultat' von pracowal 'arbeiten' mit den Merkmalen [ \pm ABSTRAKT], anderseits aber auch den 'Verlauf', z.B. in der Redewendung: jak tam u ciebie leci praca? 'wie läuft bei dir die Arbeit?'. Die Einsetzung des Femininum 
praca für die Bezeichnung des 'Verlaufs' scheint die gleiche Begründung $z u$ haben wie die Einsetzung der 'Verlaufs'-Nomina für die Bezeichnung des 'Resultats' im Falle (a), nämlich das Fehlen einer lexikalischen Repräsentation vom TYP (150); in DOR wird zwar das Nomen pracowanie als nominalisiertes Verb gebucht, jedoch in keinster weise belegt. Einer Gegenprobe zu zwqtpienie entspricht das deperfektive obszuzenie 'Bedienen', das sich in seiner Bezeichnung des 'Verlaufs' deutlich von obszuga 'Bedienung', also vom 'Resultat', abhebt, wobei allerdings auch keine resultative Aktionsart vorliegt. ${ }^{86}$

Aus den in (a) und (b) genannten Gründen muB hier also die Möglichkeit eingeräumt werden, daB einige Neutra in (150), die von perfektiven Verbalstämmen gebildet werden, auch das 'Resultat' bezeichnen können; doch gemessen an der Tendenz, die sich in der Affigierung der Verben zeigt, ist diese Bezeichnungsfunktion der Neutra (150) eine sekundäre; denn an erster stelle steht die Bezeichnung des 'Verlaufs', wobei die dem jeweiligen Verbalstamm zugrundeliegende Aktionsart, sofern sie nicht die Merkmale [- EINMALIG] sowie [- WIEDERHOLT] betrifft, in die Bezeichnung mit einbezogen werden kann.

In die aufzustellenden AF-Regeln für die Bildung der Neutra (150) werden also die Aspektmerkmale der Verbalstämme sowie die Merkmale (145(ii)) aufgenommen.

Für die Bildung der Neutra $(150(i)-(i v))$ gilt folgende AF-Regel: (AF 4.6.2)

$$
\begin{aligned}
& {\left[\begin{array}{l} 
\pm \text { IMPERFEKTIV } \\
- \text { ITERATIV }
\end{array}\right]}
\end{aligned}
$$

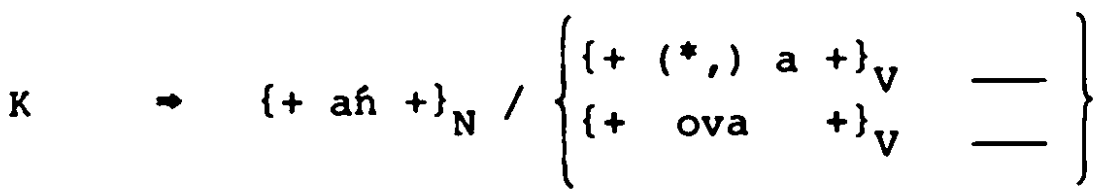

$$
\begin{aligned}
& {\left[\begin{array}{l}
- \text { BELEBT } \\
\pm \text { ZUSTAND } \\
+ \text { VERLAUF } \\
- \text { WIEDERHOLT } \\
- \text { EINMALIG } \\
+ \text { NEUTRUM }
\end{array}\right]}
\end{aligned}
$$

Für die Bildung der Neutra $(150(v)-(v i i i))$ dagegen gilt folgende AF-Regel: 
(AF 4.6.3)

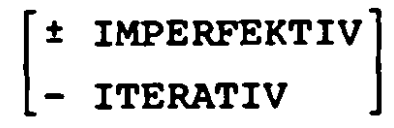

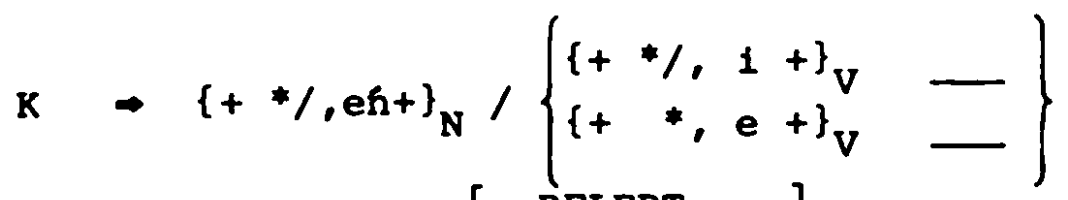

$$
\begin{aligned}
& {\left[\begin{array}{l}
- \text { BELEBT } \\
\pm \text { ZUSTAND } \\
+ \text { VERLAUF } \\
\text { - WIEDERHOLT } \\
\text { - EINMALIG } \\
+ \text { NEUTRUM }
\end{array}\right]}
\end{aligned}
$$

Der Unterschied 2 wischen den Regeln (AF 4.6.2) und (AF 4.6.3) besteht ausschlieblich in der morphonemischen Repräsentation des jeweiligen nominalen Klassifikators, die durch die morphonemischen Repräsentationen des verbalen $T M$ bedingt ist.

Zusammenfassend läBt sich zur Bildung der Neutra (149) und der Neutra (150) folgendes feststellen:

(149.1) (a)Die Neutra (149) sind die imperfektiven Gegenstücke zu (150.1) den Neutra (148), d.h. sie werden von imperfektiven Verbalstămnen gebildet, während die Neutra (150) sowohl von perfektiven als auch von imperfektiven verbalstämmen gebildet werden;

(b) Die Neutra (149) werden von Verbalstämmen mit der TM-Repräsentation $\{+\varnothing+\}$ gebildet, wăhrend die Neutra (150) von den Verbalstămnen der verben auf -as, -owac sowie auf $-i c /-y c,-e c$ gebildet werden;

(c) In der Regel bezeichnen die Neutra (149) und (150) den 'nicht wiederholten' und 'nicht einmaligen verlauf' entsprechend (145(ii)); wird das 'Resultat' durch kein Wort vom Typ (146) oder (147) repräsentiert, so können die Neutra (149) und (150) vereinzelt auBer den Merkmalen (145(1i)) auch den Merkmalen (145(1)) zugeordnet werden; für die Neutra (150) gilt auch dann 'Resultat', wenn dem Verbalstamm die resultative Aktionsart zugrunde liegt;

(d) Die unterschiedliche nominale $k$-Repräsentation beider Gruppen ist durch die unterschiedliche TM-Repräsentation der Verbalstäme bedingt;

(e)Für die phonologische Endableitung gilt u.a. (DL29(1)). 
Das Merkmal [- WIEDERHOLT] gilt auch für die Neutra, die ausschlieblich von den Verben auf -qc gebildet werden. Auch diese Neutra können vereinzelt neben den Merkmalen (145(iii)) den Merkmalen (145(i)) zugeordnet werden. In (148) sind bereits zwei solcher Beispiele genannt, die ebenfalls zu den Neutra (151) gezählt werden können.

(151) rqbniecie 'Schlag' stanifcie 'Stehenbleiben' rykniqcie 'Aufbrüllen' agiecie 'Biegung' wstraq\&niecie 'Brschütterung' padniecie 'Hinfallen' wykrzykniqcie 'Asrufen' bzysiniecie 'Aufblitzen' zabzyoniecie 'Brblitzen' odbzyóniecie 'Blinken' cofnięcie 'Rückzug' zamknięcie 'Schließung'

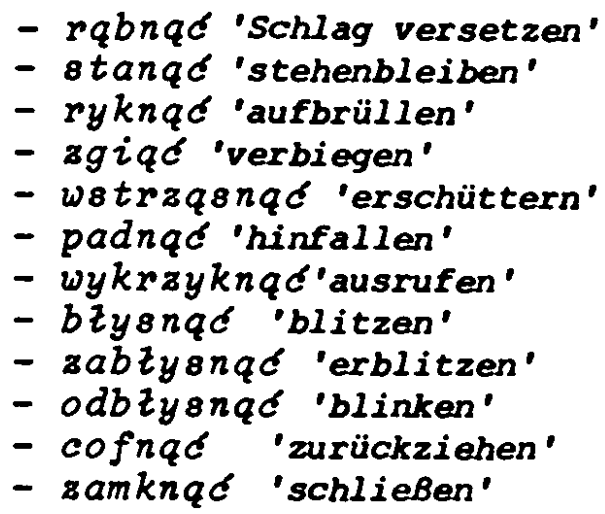

Für all die Fälle, in denen die Neutra (151) ebenfalls das 'Resultat' im Sinne der Merkmale (145(i)) bezeichnen, gilt die Regel (AF 4.5.2): sonst gilt folgende AF-Regel:

(AF 4.7)

$$
\begin{aligned}
K \rightarrow\{t, \tilde{e} c+\}_{N} /\left\{\begin{array}{l}
\{+\mathrm{n}+\}_{\text {IF }} \\
\{+j+\} \mathrm{TF}
\end{array}\right\}\{+\tilde{o}+\} \mathrm{V}- \\
\\
{\left[\begin{array}{l}
- \text { IMELERTERTIV } \\
\pm \text { ZUSTAND } \\
+ \text { VERLAUF } \\
- \text { WIEDERHOLT } \\
+ \text { EINMALIG } \\
+ \text { NEUTRUM }
\end{array}\right] }
\end{aligned}
$$

Die Annahme der TF-Repräsentation $\{+j+\}$ TF in (AF 4.7) ist $z . B$. durch agiecie bedingt, dem die morphonemische stamm-Repräsentation $l_{V}=z-g i+j+\tilde{o}+$ ] zugrunde liegt; wollte man auf dieses TF verzichten, so ergäbe die Nominalisierung - infolge der Einsetzung des Operators $Q$ entsprechend (DL29(1)) - ein nicht existierendes *zgecie. Die Annahme des IF in (AF 4.7), das auch in der graphemischen Repräsentation vorhanden ist, entspricht der Assoziation des Verbalstammes mit dem Merkmal [+ EINMALIG]. Zusammenfassend läßt sich zur Bildung der Neutra (151) folgendes 
feststellen:

(151.1) (a)Die Neutra (151) werden ausschlieblich von semelfaktiven Verben auf $-(n)-q$ f gebildet;

(b) In der Regel werden die Neutra (151) durch die Merkmale (145(1ii)) gekennzeichnet; haben die Merkmale (145 (i)) keine lexikalische Reprăsentation vom Typ (146) bzw. (147), so können die Neutra (151) zusätzlich das 'Resultat' bezeichnen;

(c) Gegenüber den Neutra (150) sind die Neutra (151) bezüglich [+ EINMALIG] markiert;

(d) Für die morphonemische Repräsentation des Verbalstammes wird entweder das TF $\{+j+\}$ oder das IF $\{+n+\}$ angenommen;

(e)Für die phonologische Endableitung gilt u.a. (DL29(1)).

Schlieblich sollten hier noch die Neutra genannt werden, die ausschlieblich von iterativen verbalstämmen gebildet werden und die Merkmale (145(iv)) tragen. Zu diesen Neutra gehorren u.v.a.:

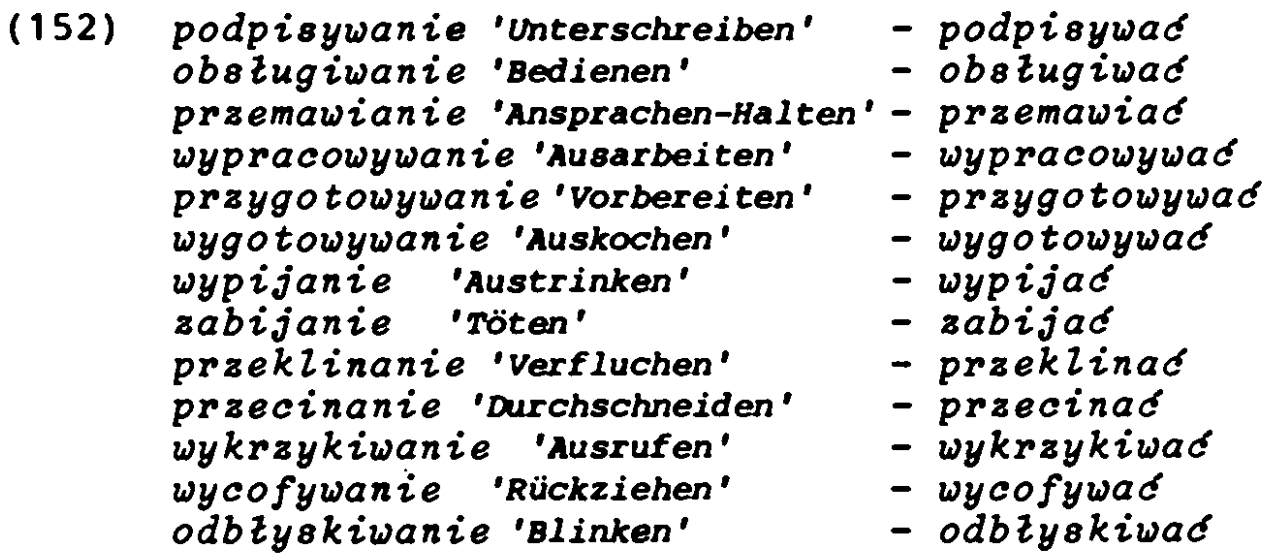

Die deutschen Entsprechungen in (152) sind nicht sehr genau: so kann z.B. das Nomen podpisywanie durch 'Unterschriften-Leisten, Zu-Unterschreiben-Pflegen' wiedergegeben werden, oder przygotowywanie durch 'Vorbereitungen-Treffen'. Es handelt sich in allen Fällen um Iterativa bzw. Imperfektiva, die auch als Iterativa gebraucht werden. Die Kennzeichnung des Iterativs wird in den meisten Fällen durch ein IF bestimmt, das den graphemischen Repräsentationen - $i w-/-y w$ - oder -aw-zugrunde liegt; in przeklinanie und przecinanie liegt jewells eine $\emptyset / i-A l t e r n a t i o n$ vor, nämlich im Verbalstamm, vgl. przekinnqd, przethnq̨, deren Realisation als $\{i\}$ mit dem Iterativ assozilert wird. 
Für die Neutra (152) kann folgende AF-Regel aufgestellt werden:

(AF 4.8)

$$
\begin{aligned}
& {\left[\begin{array}{l}
+ \text { IMPERFEKTIV } \\
+ \text { ITERATIV }
\end{array}\right]} \\
& K \quad \Rightarrow \quad\{+a \hat{f}+\}_{N} /\left\{\begin{array}{l}
\{+i v+\} \text { IF } \\
\{+a v+\} I F \\
\{+1 j+\} \text { IF }
\end{array}\right\}\{+a+\} v- \\
& {\left[\begin{array}{l}
- \text { BELEBT } \\
\pm \text { ZUSTAND } \\
+ \text { VERLAUF } \\
+ \text { WIEDERHOLT } \\
+ \text { NEUTRUM }
\end{array}\right]}
\end{aligned}
$$

Zusammenfassend läBt sich zur Bildung der Neutra (152) folgendes feststellen:

(152.1) (a)Die Neutra (152) werden ausschlieblich von iterativen Verbalstämmen gebildet;

(b) Gegenüber allen anderen deverbalen Neutra, Feminina und Maskulina, die durch die Merkmale (145) gekennzeichnet sind, sind die Neutra (152) bezüglich [+ WIEDERHOLT] markiert;

(c) Die Iterativität der Verbalstämme wird durch eine entsprechende IF-Repräsentation bestimmt; im Falle der Verbalstămme, wie z.B. wybijanie wird die IF-Reprăsentation $\{+1 j+\}$ IF angenommen:

(d) Fur die phonologische Endableitung gilt u.a. (DL29(1)). 
Die Nominalisierung im Polnischen wird hier als ein Proze $B$ dargestellt, der auf die Voraussetzungen der generativen Grammatik gestützt ist. Demnach wird das $z u$ bildende Nomen in die S-Basis eingesetzt, wo auch die DER-Regeln und die AF-Regeln angewandt werden. Die Anwendung dieser Regeln schlieBt die Anwendung der phonologischen Alternationsregeln ein, da die Nominalisierung im Polnischen in der Regel mit phonologischen Alternationen verbunden ist. Den DER-Regeln, den AF-Regeln und den PL-Regeln liegt die morphosyntaktische Struktur (61) zugrunde. Mit Hilfe der morphosyntaktischen struktur und der drei Regeltypen wird die W-Basis des $z u$ bildenden Nomen erzeugt. Die W-Basis enthält phonologische, morphologische und inhärente Merkmale, darunter auch die Genus- und die lexikalischen Kategorienmerkmale. W-Basis und S-Basis stellen zwei von einander getrennte strukturen dar: denn während die S-Basis von $\bar{S}$ dominiert wird, wird die $W$-Basis des Nomen von $\mathrm{N}$ dominiert. Innerhalb der $\mathrm{W}$-Basis wird der Wortstamm des zu bildenden Nomen von $C$ regiert, das Suffix bzw. der nominale Klassifikator wird von $K$ regiert und von $C$ präzediert. Der Klassifikator ist gegenüber dem stamm-Marker immer markiert. $C$ und $k$ werden vom lexikalischen Formationsteil (LFT) bzw. vom Wortbildungsteil (WT) der W-Basis - entsprechend (47.0) - dominiert. C dominiert auch die potentielle oder aktuelle wortverbindung, die mit dem $z u$ bildenden Nomen das von $C$ regierte Nort gemeinsam haben kann. Sowohl der potentiellen oder aktuellen wortverbindung als auch dem nominalen Klassifikator werden die inhärenten Merkmale zugeordnet.

\subsection{ZUR EINBEZIEHUNG DER WORTVERBINDUNGEN}

Die Einsetzung des LFT in $\mathrm{N}$ - vgl. (47.0) - sowie die Ersetzung des zu bildenden Wortstammes durch $C$ in (15) geben die Möglichkeit, die wortverbindung in die Wortbildung einzubeziehen; vgl. $\S 5.4 .1$ und $\S 5.4 .2$. Dabei erweist sich die Kategorie $t_{i}$ insofern nützlich, als mit ihrer Hilfe die Merkmale, mit denen im affixorientierten Polnisch der Klassifikator assoziiert wird, auf dessen morphonemische Repräsentation übertragen werden können. Dabei ist es gleich, welche syntaktische struktur die Wortverbindung hat; die Relation zwischen Regieren und Präzedenz ennöglicht auch 
in der C-Struktur die Einsetzung der Konstituenten im Hinblick auf die $W$-Basis sowohl des zu bildenden Nomen als auch der $z u$ bildenden Wortverbindung, wobei der letzte terminale knoten, der als einziger von $C$ regiert wird, dem unmarkierten wortstamm des zu bildenden Nomen - vgl. (47.1), (47.4.1)-(47.4.3) - entspricht. Bei der Einsetzung der Wortverbindung in $C$ handelt es sich jedoch um keine Transformation im satzsyntaktischen Sinne; es handelt sich hier allein um die zuordnung der sowohl anhand der Wortverbindung als auch anhand des zu bildenden Nomen assoziierbaren Merkmale, die beiden, d.h. der Wortverbindung und dem $z u$ bildenden Nomen gemeinsam sein können.

Die Einbeziehung der Wortverbindung trägt der Forderung Laskowskis - vgl. S. 54 - nach einer transformativ-syntaktischen wortbildung im Polnischen Rechnung. Man kann nämlich nicht umhin, feststellen zu müssen, daß z.B. Lakiernia und fabryka lakierbw 'Lackfabrik' als gleichbedeutende lexikalische Einheiten gebildet und - ähnlich wie diedeutschen Nomina Dampfschiff und Dampfer - in denselben Endknoten der S-Basis eingesetzt werden können. Wollte man die Wortverbindung aus der Wortbildung ausschließen, so müBten auch solche lexikalische Einheiten unberücksichtigt bleiben, wie z.B. pasta do zebbw'(wörtl.) Pasta für die Zähne = Zahnpasta' oder szczotka do butbw '(wörtl.) Bürste für die Schuhe = Schuhbürste', denen keine einstämmigen Nomina entsprechen.

Vergleicht man in $\S 8$ die Merkmale in den AF-Regeln mit den potentiellen oder aktuellen Wortverbindungen bzw. Paraphrasen, so läßt sich feststellen, daß zwischen dem zu affigierenden Nomen und scinen inhärenten Merkmalen das Verhältnis der Zuordnung herrscht; dieses Verhältnis der Zuordnung muß aber auch für die Beziehung zwischen der Wortverbindung und den inhärenten Merkmalen angenommen werden, wenn Nomen und Wortverbindung die gleiche Bedeutung bei unterschiedlicher morphosyntaktischer struktur haben. So werden z.B. die Merkmale [+ BELEBT; + MENSCHLICH] entweder einem 'belebten, menschlichen' Nomen im markierten Teil der wortverbindung oder einer entsprechenden $K$-Repräsentation zugeordnet, das Merkmal [+ RÄUMLICH] wird entweder einem 'räumlichen' Nomen oder dem Suffix - $n i-$, das Merkmal [+ ZUGEHठRIGKEIT] wird entweder den Präpositionen $d l a$, od 'für' oder einem ent- 
sprechenden Suffix zugeordnet. Im Falle der Wortverbindung fabryka lakierow mübte man sogar von einem inhärenten GENITIVUS ausgehen, der im Gegensatz zum POSSESSIVUS, der nur 'belebte' Nomina betrifft, und im Gegensatz zum PARTITIVUS, der nur 'nicht zählbare' Nomina betrifft, einen 'Teil' des davorstehenden Nomen bezeichnet ${ }^{87}$; die Tatsache, daß jedoch dieser GENITIVUS mit dem GEN der S-Basis nichts zu tun haben kann, zeigen die möglichen Einsetzungen dieser Wortverbindung, die der Einsetzung $z$. B. von zodí zaglowa 'Segelboot' - vgl. $§ 5.4 .2$ - entsprechen, d.h.. daB diese Wortverbindung wie ein Nomen in die S-Basis eingesetzt wird.

Auf Grund dieser Feststellungen kann man behaupten, daB Nomen und Wortverbindung eine gemeinsame Bedeutung haben können, während ihre morphosyntaktischen strukturen unterschiedlich realisiert sind. Ungeachtet der Tatsache, daB es Nomina ohne eine entsprechende, aktuelle Wortverbindung und daB es Wortverbindungen ohne ein entsprechendes, aktuelles Nomen geben kann, muB für all die Fälle, in denen beide Repräsentationen vorhanden sind, eine gemeinsame C-Struktur angenommen werden, die beide Repräsentationen für die zuordnung der inhärenten Merkmale in sich vereint: vgl. auch (49). Da jedoch Wortverbindung und Nomen unterschiedliche morphosyntaktische strukturen haben, werden sie auch anhand unterschiedlicher DER-Regeln - vgl. (50.1) und (50.2) - sowie unterschiedlicher AF-Regeln gebildet. Es ist nämlich ein Unterschied, ob der Sprecher zठdz zaglowa aus zwei Wortstämmen bildet, indem er das Nomen vor das Adjektiv stellt und durch entsprechende suffixe u.a. lexikalisch klassifiziert, oder ob er zaglowka aus einem Wortstamm bildet, indem er dem adjektivischen stamm-Marker das nominale suffix hinzufügt.

Im Zusammenhang mit der sog. Univerbierung wurden bereits Beispiele genannt, die keiner aktuellen Wortverbindung - vgl. S. 68 - entsprechen; so 2.B. ciqzarobka (FEM) 'Lastkraftwagen'. Die Wortverbindung mit derselben Bedeutung wäre samochod ciqzarowy (MAS), demnach müBte - der Annahme Laskowskis (siehe: S. 56) zur Folge - das univerbierte Nomen *cifiarowiec (MAS) heiBen. Doch ciezarowiec hat die Bedeutung von 'Schwerathlet'. Für die Bildung des Femininum ciezarówka, dem keine aktuelle, im Genus übereinstimmende Wortverbindung entspricht, können zwei Möglich- 
keiten angenommen werden, nämlich:

(a) ciezarowka wird in Analogie zu den 'Fortbewegungsmitteln' (131) gebildet;

(b) ciezarowka wird eine c-struktur zugrunde gelegt, deren markiertes $\mathrm{N}$ nicht lexikalisch, sondern ausschlieblich durch Merkmale repräsentiert ist.

Im Falle der Analogie, d.i. (a), würde das heiBen, daß die Assoziation der Affixe mit den Merkmalen der 'Instrumentalität' und des 'Fortbewegungsmittels' nebst Genus bereits keines entsprechenden Nomen einer Wortverbindung bedarf, um das entsprechende Nomen bilden zu können. Im Falle (b) könnte man von folgender Voraussetzung ausgehen, nämlich:

$$
\begin{aligned}
& \mathrm{N}_{i} \text { cieziarow }-t_{i} \\
& \text { WENN : } i \quad\left[\begin{array}{l}
- \text { BELEBT } \\
+ \text { INSTRUMENTALITAT } \\
+ \text { FEMININUM }
\end{array}\right]
\end{aligned}
$$

dies unter Einbeziehung der Merkmale (107). Doch stellt sich bei dieser Lösung die Frage nach der Begründung der Annahme solch einer C-struktur, wenn es keine lexikalische Repräsentation dafür gibt.

Das Femininum cipzarókka ist also ein Beispiel dafür, das die Wortverbindung nicht als Voraussetzung für die Bildung eines Nomen gelten kann; der Wortverbindung kann, sofern sie vorhanden ist, dieselbe Bedeutung, d.i. dieselben inhärenten Merkmale wie dem Nomen zugeordnet werden, doch gelten für ihre Bildung - wie bereits festgestellt - andere Regeln als für die Bildung des Nomen. Daher muB im Falle des Femininum ciezarowka ebenso wie im Falle aller anderen Nomina davon ausgegangen werden, daB deren W-Basis völlig unabhängig davon, ob es eine aktuelle oder potentielle Wortverbindung gibt oder nicht, mit den entsprechenden inhärenten Merkmalen, die für das jeweilige Nomen gelten, assozilert wird. Anderseits gibt es nämlich Wortverbindungen, die völlig unabhängig davon, ob sie univerbiert werden können oder nicht, mit den entsprechenden inhärenten Merkmalen assoziiert werden, wie z.B. die bereits genannten Beispiele vom Typ szczotka do butow oder dom towarowy 'Warenhaus'; in diesen wortverbindungen gibt es keine Möglichkeit, den unmarkierten, von $C$ 
regierten Teil der C-struktur bei gleichbleibender Bedeutung $z u$ affigieren.

Die Unabhängigkeit der Affixe von potentiellen oder aktuellen Wortverbindungen bzw. Paraphrasen wird u.a. in der Bildung der demaskulinen Feminina, wie z.B. in (119), deutlich. Wollte man nach dem Muster der Maskulina (118) für die Feminina eine Paraphrase vom Typ kobieta/ta, co pracuje w urzedzie 'Frau/eine, die im Amt arbeitet' annehmen, so müßte man *urzedka statt urzednicz$k a$ bilden. Als mögliche Wortverbindung gälte kobieta urzednik 'Frau als Beamter', die zwar grammatikalisch richtig, jedoch nicht gebräuchlich ist. Statt der Einsetzung solch einer wortverbindung, die keiner Aktualität entspricht, kann man sich auf die Feststellung stützen, die der morphonemischen Repräsentation der Affixe entspricht, daB die Feminina (119) von den Maskulina (118) mit Hilfe des femininen Klassifikators $\left\{{ }^{*} \& \mathrm{k}+\right\}_{\mathrm{N}} \mathrm{ge}-$ bildet werden und daß diese Feminina bezüglich dieses Klassifikators gegenüber den Maskulina (118) markiert sind, d.h., daB sie sich sonst in keinem anderen inhärenten Merkmal von einander unterscheiden.

Die Ablehnung des Algorithmus von Laskowski - vgl. S. 56 - und damit einer "transformativ-syntaktischen' Wortbildung im Polnischen wird also auf folgende Feststellungen gestützt:

(a) Wortverbindung und Nomen können ein und dieselben inhärenten Merkmale haben;

(b) Wortverbindung und Nomen können, wenn ihnen ein und dieselben inhärenten Merkmale zugeordnet werden, eine gemeinsame C-struktur haben, weil sie u.a. auch beide in denselben Knoten der $\mathrm{S}$-Basis eingesetzt werden;

(c) es gibt jedoch Nomina, die im Genus mit der potentiellen oder aktuellen wortverbindung nicht übereinstimmen;

(d) es gibt Nomina, denen keine potentielle oder aktuelle Wortverbindung entspricht;

(e) es gibt Wortverbindungen, denen kein Nomen entspricht;

(f) Wortverbindung und Nomen werden anhand unterschiedlicher DER-Regeln und AF-Regeln gebildet.

Wollte man für die Nominalisierung Univerbierungen annehmen, so müBte man auch Transformationen im LFT annehmen; solche Transformationen wären theoretisch möglich, doch widersprächen sie 
den genannten Gegebenheiten des Lexikons im Polnischen.

Die in $\S 5.4 .1$ und $\S 5.4 .2$ eingesetzten wortverbindungen bzw. Paraphrasen haben im Hinblick auf die Nominalisierung im Polnischen den Status von lexikalischen Belegen für die inhärenten Merkmale, die der Sprecher des Polnischen neben den zu bildenden Nomina realisieren kann. Doch da diese Wortverbindungen bzw. Paraphrasen nicht immer $z u$ bilden sind oder da diese Paraphrasen bzw. Wortverbindungen auch ohne entsprechendes Nomen gebildet werden, sind Nominalisierung und Bildung von Wortverbindungen trotz möglicher gemeinsamer C-Struktur als zwei getrennte prozesse innerhalb der Wortbildung des Polnischen zu sehen; daher werden auch die inhärenten Merkmale in $\S 7.2 .4$ unabhängig von potentiellen oder aktuellen Wortverbindungen bzw. Paraphrasen, also unabhängig vom markierten Teil der C-Struktur, die den zu affigierenden Wortstamm präzediert, definiert.

\subsection{ZUR MORPHOSYNTAKTISCHEN STURKTUR}

Aus den in $\S 8$ dargestellten Nominalisierungen geht u.a. die Affixorientiertheit des Polnischen hervor. Während man im Deutschen häufiger das Kompositum vorfindet, so z.B. in Stahlarbeiter, Glühbirne, Zuckerdose, findet man im Polnischen die einstämmigen affigierten Entsprechungen stal-owi-ec, $\dot{z} a r-\sigma w-k-a$, cukier-nic-a. Im Polnischen werden also dort Affixe gesetzt, wo im Deutschen ein zweiter Wortstamm steht.

Die Tatsache, daß im Polnischen auf den zweiten Wortstamm - Komposita ${ }^{8 \theta}$ sind hier ausgenommen - verzichtet werden kann, ist sowohl durch die Position als auch durch die Funktion der Affixe bedingt. In stal-owi-ec steht das adjektivische Affix zwischen dem nominalen stal- und dem nominalen klassifikator -ec, um zwischen beiden eine morphonemische verbindung zu schaffen, diemit der 'zugehörigkeit' des 'belebten, maskulinen Trägers' zum 'Stahl' assozilert wird. Die Assoziation mit dem Merkmal der 'zugehörigkeit' ist aber Folge der Funktion des adjektivischen Affixes als Stamm-Marker, der vom Klassifikator durch die Alternation $\{v\}$ vs. $\left\{v^{\prime}\right\}$ getrennt ist, während seine vordere Grenze durch die vokalische Alternation $\left\{\&_{3}\right\}$ bestimmt wird. Bei der Segmentierung von /stal' $\& v^{\prime} 8 \mathrm{c} /$ in Wurzel und Affixe sind 
also einerseits die phonologischen bzw. morphonologischen Alternationen zu berücksichtigen, anderseits die Stellung der Affixe bezüglich der wurzel und bezüglich ihrer selbst; denn sobald die Positionen der Affixe vertauscht würden, entstünden Nonsensbildungen. Deshalb werden die Affixe entsprechend ihrer morphosyntaktischen Position innerhalb der äuBeren und der inneren Grenzen - wie sie in (13) definiert sind - um die Wurzel herum gruppiert, damit sie dem jeweiligen Merkmalkomplex sinnvoll zugeordnet werden können; und dies geschieht mit Hilfe der jeweils zuständigen AF-Regel, die auch die Affixgrenzen berücksichtigt.

Bevor jedoch die jeweilige AF-Regel aufgestellt werden kann, werden die in (13) definierten wortgrenzen in den LFT und GFT in (15) eingesetzt. Auf Grund der unterschiedlichen Position und der unterschiedlichen Funktion der Affixe werden in $\S 6$ Morphem-Marker bestimmt, um mit deren Hilfe die aus (13) und (15) resultierende morphosyntaktische struktur (61) jedem $z u$ bildenden - und zu beugenden - Nomen zugrunde zu legen. Für Nomina, die von einem lexikalisch bereits kategorisierten wortstamm gebildet werden - und dazu gehören die meisten - gilt zusätzlich (DL 30) nebst anderen Tilgungsregeln. Die morphosyntaktische Struktur (61) wird den DER-Regeln und den AF-Regeln zugrunde gelegt. Die morphemmarkierte morphosyntaktische struktur trägt also der Affixorientiertheit des Polnischen insofern Rechnung, als sie die Möglichkeit schafft, die Affixe entsprechend ihrer Funktion der Assoziation mit bestimmten inhärenten Merkmalen in die richtige Position der W-Basis einzusetzen.

Die Bestätigung für die Richtigkeit der Annahme einer morphemmarkierten morphosyntaktischen Struktur geben u.a. Fälle, in denen ein und dieselbe morphonemische Repräsentation zwei unterschiedliche Funktionen hat, wie z.B. $\{+* 8 \mathrm{k}+\}$. Dieses Morphem repräsentiert einerseits den nominalen klassifikator der entsprechenden Feminina, vgl. urzednicz-k-a, anderseits aber den diminutiven Evaluator, z.B. im Femininum dojarecz-k-a von dojar$k a$ 'Melkerin' oder im Maskulinum domek von dom 'Haus'. Uber die Funktion als Klassifikator oder als Evaluator kann hier nur die Position bezüglich der anderen Morphem-Marker innerhalb der morphosyntaktischen Struktur (61) entscheiden: denn der Evaluator setzt im Polnischen stets die Realisierung eines Klassifikators 
voraus. Im Diminutiv dojareczka wird diese morphonemische Repräsentation zweimal eingesetzt, einmal als femininer nominaler Klassifikator und anschlieBend als diminutiver Evaluator, der an den inhärenten Merkmalen des mit dem Klassifikator abgeschlossenen Nomen nichts verändert.

\subsection{ZUR AFFIGIERUNG}

An der Affigierung der Nomina im Polnischen sind die phonologischen Alternationen, die Genusmerkmale, die lexikalischen Kategorienmerkmale sowie die inhärenten Merkmale beteiligt.

Die phonologischen Alternationen werden - in Anlehnung an Baudouin de Courtenay (1895) - hier zu den korrelierenden, im Gegensatz $z \mathrm{u}$ den phonetisch bedingten divergierenden Alternationen gezählt. Den korrelierenden, d.i. an der Wortbildung beteiligten Alternationen entsprechen die $\mathrm{PL}-$ Regeln in $\S 7.1 .2$.

Anhand der PL-Regeln sowie anhand des merkmalphonologischen Inventars (68) läßt sich feststellen, daß die velaren Konsonanten mit den alveolaren bezüglich [ \pm VORN], die dentalen mit den alveolaren Konsonanten sowie die beiden nichtlateralen Liquida bezüglich [ \pm ANTERIOR] und die übrigen Konsonanten bezüglich [ \pm HOCH] korrelieren. Demnach gelten für die grenzmarkierenden Alternationen in der Nominalisierung des Polnischen:

(154)(i) $\underline{C}(*)$ impliziert [ \pm ANTERIOR] /

[- DAUERND]

(ii) $\quad \underline{C}\left(^{*}\right)$ impliziert $[ \pm$ VORN]/ SONST

(iii) $\quad \underline{c}($,$) impliziert [ \pm$ ANTERIOR] /

$\left[\begin{array}{l}- \text { OBSTRUENT } \\ - \text { LATERAL }\end{array}\right]$

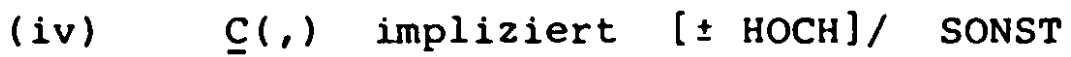

Die konsonantischen Alternationen (154) bestimmen in der Regel jeweils die hintere Grenze des affigierten sowie die vordere Grenze des affigierenden Morphem-Markers, bzw. dessen morphonemischer Repräsentation, so z.B. in nart-y - narci-arz-narci-ar$-k-a$ - narci-ar-ecz-k-a Schier-Schifahrer-Schifahrerin-(DIMIN) Schifahrerin'; der nominale Stammauslaut wird durch die Alternation [ $\pm \mathrm{HOCH}]$, der Affixauslaut des maskulinen Nomen agentis durch [ \pm ANTERIOR], der Affixauslaut des femininen Pendants durch [ \pm VORN] bestimmt. Die Alternation [ \pm HOCH] kennzeichnet 
also in nart- / narci- den Auslaut des nominalen Wortstammes, der entweder als Nomen oder als affigierter Nominalstamm eingesetzt wird, die Alternation [ \pm ANTERIOR] in narciarz / narciarkennzeichnet den Unterschied zwischen maskulinem Nomen agentis und dessen Funktion als nominaler wortstamm, der entweder feminin nominal, sächlich nominal oder adjektivisch affigiert wird, die Alternation [ \pm VORN] in narciark-a / narciareczk-a kennzeichnet schlieblich das demaskuline feminine Nomen agentis und dessen Dininutiv.

Die korrelierenden Vokale sind in der nominalen Affigierung auf die Alternationen $\{\&\}$ und $\left\{\&_{3}\right\}$ beschränkt, d.h. innerhalb der Affixe treten nur diese beiden Alternationen auf, wăhrend die anderen Vokalalternationen in der wortwurzel realisiert werden. Für die korrelierenden Vokale in den Affixen gelten also:

$$
\begin{array}{ll}
\text { impliziert } & {[ \pm \text { SILBISCH }]} \\
\text { impliziert } & {[ \pm \text { HOCH }]}
\end{array}
$$

d.h., das der sog. flüchtige Vokal entweder als Vokal oder als Null, die u/o-Alternation dagegen entweder durch das hohe $\{u\}$ oder durch das nichthohe $\{0\}$ realisiert werden.

Zwischen den im Affix korrelierenden Vokalen und Konsonanten gibt es nur bei der $\left\{\varepsilon_{3}\right\}$-Alternation eine Beziehung; vergleicht man z.B. motor-ow-y - motor-owi-ec - motor- $b w-k-a$ 'Motor-, Motorschiff, Motorboot' oder wagon-ow-y - wagon-ow-ni-a 'Waggon-, Waggonhalle' so kann man feststellen, daß der vokalische Alternant $\{u\}$ nur vor dem nichtpalatalen $\{k\}$ realisiert wird, vor den anderen palatalen Konsonanten bzw. vor Vokalen, die keine Alternanten der Alternation $\{\xi\}$ sind, dagegen nicht: vgl. dazu (PL19).

Die vokalische Alternation (155(i)) steht in keiner Beziehung zu den alternierenden Konsonanten; sie steht in Beziehung zum nachfolgenden Silbennukleus: vgl. dazu (PL16). Also gilt:

$$
\begin{aligned}
& \text { (156)(i) }\{8\} \rightarrow[+ \text { SILBISCH }] / \longrightarrow[- \text { SILBISCH }] \\
& \text { (ii) }\{\&\} \rightarrow[- \text { SILBISCH }] / \longrightarrow \text { [+ SILBISCH }]
\end{aligned}
$$

Demnach wird der vokalische Alternant $\{8\}$ entweder durch einen nachfolgenden Alternanten von $\{\xi\}$ oder durch einen nachfolgenden Vokal oder aber durch einen "Null"-Alternanten bedingt. 
Die Affigierung zeigt eine starke orientierung am Genus, d.h., daB die Zuordnung des jeweiligen Genus der morphonemischen Repräsentation der nominalen Affixe entspricht. Man vergleiche:

MAS

FEM

NEU

$$
\left\{\begin{array}{l}
+a k+ \\
+a \dot{c}+ \\
+(n), i k+ \\
+(*,) a \dot{r}+
\end{array}\right\}
$$

$$
\begin{aligned}
& \{+* 8 k+\} \\
& \{+, 8 \mathrm{~h}+\} \\
& \{+* \text { if }+\} \\
& \{+(n) * \text { ict }\}
\end{aligned}
$$

$$
\{+*, 8 c+\}
$$$$
\text { (iii) }\{+*, 8 \mathrm{~h}+\}
$$

$$
\begin{aligned}
& \{+\underline{G}+\} \\
& \{+, \tilde{e} \varepsilon+\} \\
& \{+(*,) \emptyset+\}
\end{aligned}
$$$$
\text { (v) }\{+\varnothing+\}
$$$$
\{+\varnothing+\}
$$

Die nominalen Affixe (157) - vgl. dazu die AF-Regeln in $\S 8$ schlieben sich sowohl gegenseitig als auch bezüglich der drel Genera aus, d.h. jedes Affix hat seine eigene morphonemische Repräsentation, auf Grund welcher es einem der drei Genera unverwechselbar zugeordnet werden kann. Eine Ausnahme bildet der "Null"-Klassifikator, der für alle drei Genera gilt und u.a. damit zusammenhängt, daB auch die polnischen Nominalstämme ausschlieblich konsonantisch auslauten. Die Genuszuordnung übernimmt hier das NOM-SG-Flektiv, das in der Regel einem "Null"-Flektiv bei den Maskulina, einem - $a$ bei den Feminina und einem -o bei den Neutra entspricht; im Falle des NOM-SG-Flektivs - $e$ bei den Neutra gilt für den Stammauslaut die konsonantische Alternation bezüglich [ $\pm \mathrm{HOCH}], \mathrm{vgl}$. tysiqclecie 'Millennium' oder

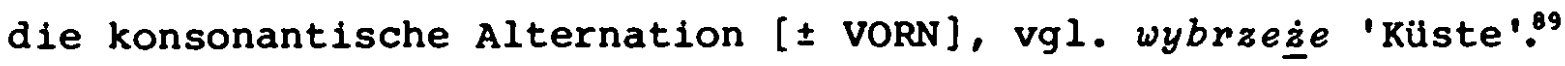
Die maskulinen Affixe (157(i)) übernehmen die Funktion von Stamm-Markern, wenn sie mit den dahinterstehenden femininen oder den dahinterstehenden sächlichen Affixen verbunden werden. Dabei bleiben bei Hinzufügung der femininen Affixe die inhärenten Merkmale des maskulinen Wortstammes entweder erhalten, wenn ein feminines Nomen agentis gebildet wird, oder das Merkmal der 'Bestimmung' wird durch das Merkmal des 'Bestimntseins' ersetzt, um entweder 'Zugehörigkeit' oder 'Instrumentalität' zu bezeichnen. In den meisten Fällen ist diese Ersetzung mit dem Genuswechsel verbunden. Die Affixe (157(ii)) und (157(iii)) betreffen hauptsächlich demaskuline, feminine Nomina agentis, wie $z . B$. 
sprzedawca - sprzedawczyni 'Verkäufer-Verkäuferin' sowie uczeñ - uczennica 'Schüler-Schülerin'. Obgleich die maskulinen Affixe (157(i)) die Funktion als Stamm-Marker erhalten, kann bei Hinzufügung des dahinterstehenden sächlichen Affixes das Merkmal des 'Trägers' - vgl. (AF 2.8.1) - zugunsten der 'Funktion' selbst negativ markiert sein; ist es positiv markiert, so bezeichnet das Neutrum ein 'Kollektivum', wie z.B. ksiggarstwo als 'Buchhänd lerschaft' .

Die Markiertheit des Genus FEMININUM - vgl. $\S 7.2 .2$ - gegenüber dem Genus MASKULINUM ist also u.a. durch die Bildung der femininen Nomina agentis von den maskulinen Pendants bedingt; und dies aufgrund der morphonemischen Repräsentation des femininen Affixes. Die Neutra mit dem Affix (157(i)) sind sowohl gegenüber den Maskulina als auch gegenüber den Feminina markiert, weil z.B. kwiaciarstwo 'Blumenhandel' sowohl vom maskulinen kwiaciarz 'Blumenhändler' als auch vom femininen kwiaciarka 'Blumenhändlerin' gebildet werden kann; auch hier entscheidet die morphonemische Repräsentation des sächlichen Affixes über die Markiertheit. Die zuordnung des Genus wäre ohne die lexikalische Kategorie nicht möglich, da nun mal die Kategorie des Genus mit der lexikalischen Kategorie des Nomen zusammenhängt, um in die stark flektierende polnische Sprache einbezogen zu werden. Aber in der Bildung der demaskulinen Feminina bzw. der demaskulinen und defemininen Neutra bleibt die zuordnung des nominal markierten Wortstammes ebenfalls erhalten, um u.a. auf die inhärenten Merkmale des maskulinen oder femininen Nomen, dessen Bildung die Bildung des Neutrum voraussetzt, hinzuweisen.

Die Kennzeichnung der lexikalischen Kategorie mittels des Affixes hängt mit der zuordnung der inhärenten Merkmale überhaupt zusammen; so wird ein verbales Affix in der Regel mit 'Tätigkeit' oder 'Zustand', ein adjektivisches Affix mit 'Eigenschaft' oder 'Zugehörigkeit', ein nominales mit 'Träger' u.s.w. assoziiert. Daher werden auch in den aufgestellten AF-Regeln stets die TM-Repräsentationen samt lexikalischer Kategorie als eine der Bedingungen für die nominale Affigierung notiert.

Die nominale Affigierung schließt also die phonologischen Alternationen als Grenzmarkierungen ein, um die morphonemisch reprä- 
sentierten Affixe um die Wurzel bzw. um den Stamm gruppieren zu können und damit die Assoziation mit den Genusmerkmalen, den lexikalischen Kategorienmerkmalen sowie den anderen inhärenten Merkmalen zu ermöglichen. Die Bedingungen für die Affigierung resultieren aus der Kombination aller hier aufgezählten und diskutierten Merkmale.

\subsection{SCHLUSS}

Die hier dargestellte Nominalisierung im Polnischen ist Teil einer Merkmal-Wortbildung, die hauptsächlich darin besteht, daß bestimmte inhärente Merkmalkombinationen bestimmten morphonemischen Repräsentationen zugeordnet werden.

Innerhalb dieser zuordnung hat die Markiertheitsrelation eine der wichtigsten Funktionen. Im Mittelpunkt scheint die Markiertheitsrelation der Genusmerkmale zu stehen; dies besonders in der Bildung der demaskulinen Feminina sowie der demaskulinen und defemininen Neutra, wie sie exemplarisch in $\S 8$ dargestellt wird. Während z.B. das feminine rzemieslniczka 'Handwerkerin' gegenüber dem maskulinen rzemieslnik 'Handwerker' allein bezüglich FEMININUM markiert ist, kommt im sächlichen rzemieslnictwo - wenn 'belebt' - neben dem Genus NEUTRUM das Merkmal des 'Kollektivum' in Sinne von 'Handwerkerschaft' - wenngleich in DOR als alte Bedeutung gekennzeichnet - hinzu, das die Merkiertheit des NEUTRUM gegenüber dem MASKULINUM bestimmt und die Bedeutung des FEMININUM mit einbezieht. Das feminine ksiggarnia 'Buchhandlung' ist gegenüber dem maskulinen ksiggarz 'Buchhändler' ebenfalls bezüglich FEMININUM markiert, wobei jedoch die zuordnung des Merkmals des 'Räumlichen' hinzukommt. Das feminine gospodarka 'wirtschaft' und das sächliche gospodarstwo 'Bauerngut' sind ebenfalls gegenüber dem maskulinen gospodarz '(u.a.)Landwirt, Hausherr' bezüglich des Genus markiert, doch dem Genusmerkmal gesellen sich andere inhärente Merkmale, die die Markiertheit gegenüber dem MASKULINUM bestimmen, hinzu.

Die Markiertheitsrelation bestimmt auch die lexikalischen Kategorienmerkmale, indem der lexikalisch kategorisierte, unmarkierte Wortstamm durch die lexikalische Kategorie $\mathrm{N}$ markiert wird. Dieser lexikalisch kategorisierte, unmarkierte Wortstamm ist auch die gemeinsame Komponente der potentiellen Wortverbindung 
und des zu bildenenden Nomen; daher sind sowohl die vor diesem unmarkierten Wortstamm stehenden Komponenten der Wortverbindung als auch das hinter diesem unmarkierten Wortstamm stehende Affix markiert. Demnach ist in kolejka wqskotorowa 'Schmalspurbahn' das Nomen kolejka, dem die Merkmale eines 'nicht belebten Funktionsträgers' in Sinne von 'Instrumentalität', d.i. 'Fortbewegungsmittel' zugeordnet werden, gegenüber dem adjektivischen Wortstamm, der einen Teil der 'Funktion' bezeichnet, markiert; wird statt dieser Wortverbindung das Nomen wqskotorowka gebildet, so ist der nominale Klassifikator $\{+* 8 \mathrm{~K}+\}_{N}$, dem dieselben inhärenten Merkmale wie der Wortverbindung zugeordnet werden, gegenüber dem adjektivischen Wortstamm markiert. Die lexikalische Kategorie $\mathbf{N}$ ist also im Hinblick auf die ihrer K-Repräsentation zugeordneten inhärenten Merkmale gegenüber der lexikalischen Kategorie des unmarkierten Wortstamms in der Nominalisierung durchweg markiert.

Bei der Einbeziehung der Wortverbindung in die Wortbildung wird die Feststellung - vgl. $\S 9.1$ - gemacht, daß Wortverbindung und Nomen ein und dieselbe Bedeutung haben können, jedoch getrennt, durch unterschiedliche Wortbildungsregeln gebildet werden. Da $\beta$ diese Feststellung keine bloBe Behauptung ist, kann auch durch solche Beipiele belegt werden, die eine sog. Univerbierung völlig ausschließen; so z.B. das Nomen miednica 'Schüssel'. Der Wortstamm entspricht dem Nomen miedi' 'Kupfer' oder dem Adjektiv miedny 'Kupfer-' - vgl. Brückner; wollte man hier von einer Wortverbindung ausgehen, so müBte diese ebenfalls ein morphonemisch nichtrepräsentiertes N - vgl. (153) - enthalten, denn im Gegenwartspolnisch gibt es keine entsprechende $\mathrm{N}$-Repräsentation. Möglich, daB es in der Etymologie dieses Nomen eine ursprüngliche $\mathrm{N}$-A-Verbindung gegeben hat, doch wie sollte das der Sprecher des heutigen Polnisch wissen. Das Nomen miednica wird allein auf Grund der inhärenten Merkmale, die dem markierten nominalen Affix zugeordnet werden mit einer 'Schüssel' assozilert, wobei es auch nähere Bezeichnungen vom Typ miednica plastykowa 'Plastikschüssel' gibt; es wäre eine grundlose spekulation, wenn man annähme, daß der Sprecher des Polnischen miednica plastykowa mit einer 'Plastik-Kupfer-Schüssel' assozilert.

Gegen die Konzeption einer "transformativ-syntaktischen' Wortbil- 
dung - vgl. $§ 5.3$ - sprechen aber auch Neubildungen aus der polnischen studentensprache, in denen es ganz offensichtlich keine Ubereinstimmung zwischen Wortverbindung und Nomen bezüglich Genus gibt; so z.B. ekonomik (MAS) für Wyisza Szkoza Ekonomiczna (FEM) 'Wirtschaftshochschule' oder waryniec (MAS) für Biblioteka im. Waryniskiego (FEM) 'Waryfski-Bibliothek'.90

Auf Grund des Dargestellten kann also festgestellt werden, daB das Affix im Polnischen weder einer Wortverbindung noch einer Paraphrase bedarf; die Kombination zwischen morphonemischer Repräsentation der Wurzel mit den lexikalischen Kategorienmerkmalen und den Genusmerkmalen der Affixe gewähren die Assoziation mit den anderen inhärenten Merkmalen, die der zu bildenden nominalen W-Basis zugeordnet werden. 
ANMERKUNGEN

1 - Entsprechend der Behauptung, die Sprache sei ein System von Zeichen.

2 - Der individuelle Charakter der Sprachverwendung wird von de Saussure parole, der soziale langue zugeschrieben.

3 - In den theoretischen Voraussetzungen in Trubetzkoy, N.S. 1939, Grundzüge der Phonologie, Göttingen ${ }^{2} 1958$.

4 - In Hjelmslev 1953.

5 - Hjelmslev (1953, § $10 \mathrm{f.})$ unterscheidet zwischen Funktiv und GröBe. Ein Funktiv entspricht dem Glied einer Kette, das zu anderen Gliedern dieser Kette in Funktion steht. Ein Funktiv, das in keiner Funktion steht, entspricht einer GröBe.

6 - Zur strukturalistischen Methode der Wortbildung siehe bes. Dokulil 1962.

7 - Zum Null-Morphem siehe: Jakobson 1939; zur Diskussion über Ye signe zéro von de Saussure siehe: Isačenko $(1961,30)$.

8 - Dazu sind besonders die Arbeiten von Ch. F. Hockett und A. Martinet zu nennen. Coseriu $(1970,112)$ stetzt das Wort lexikalischen Inhaltseinheiten gleich; diese sind im sprachlichen system enthalten.

9 - Baudouin de Courtenay wird hier referiert, weil er der modernen Sprachwissenschaft mehr zu sagen hat, als allgemein angenommen worden ist. Inzwischen ist es auch die sog. Natürliche Phonologie, die sich zu ihm bekennt. Zur Diskussion seiner Sprachtheorie siehe: Mugdan 1984; vgl. auch Pohl 1979.

10 - Die Trennung $\mathbf{z w i s c h e n ~ M a r k i e r t h e i t ~ v s . ~ U n m a r k i e r t h e i t ~ s o w i e ~}$ dem Vorhandensein vs. Nichtvorhandensein einer bestimmten Eigenschaft ist im Sinne Jakobsons (1932,II) insofern möglich, als die unmarkierten grammatischen Eigenschaften wiederum in merkmalhafte und merkmallose unterteilt werden; so wird z.B. der Aspekt des Verbs in ein merkmalhaftes Perfektiv und in ein merkmalloses Imperfektiv im Hinblick auf die Grenzen der Handlung unterteilt; das merkmallose Imperfektiv wiederum in das merkmalhafte Iterativum und in das merkmallose Nicht-Iterativum; dies im Hinblick auf die Merkmalhaftigkeit der Handlung. 
11 - Die hier verwendeten Merkmale sind ausschlieblich auf das Polnische bezogen.

12 - Zur Diskussion der Markiertheit bei Chomsky \& Halle 1968 siehe: Gardner (1975,106ff.); vgl. dazu Chomsky \& Halle (1968, 402ff.. FuBn.3f. Sowie Anwendung S. 435).

13 - Vgl. dazu bes. Koschmieder 1977, Paulsson 1979 und Pohl $1980 a$.

14 - Baudouin de Courtenay führt auch Beispiele an, in denen Alternationen dieser Art vorkommen, die nicht historisch begrüdbar sind; es handelt sich z.B. un Expressiva vom Typ nog-a $n \delta \underline{\underline{z}}-k a$ 'Bein - Beinchen'.

15 - Der Output der flektierten Seicht-Struktur entspricht dem Input der oberflächenstruktur.

16 - Wenn die lexikalische Kategorie durch Merkmal ersetzt wird, so kann das besagte Formativ eben durch das Merkmal des Knotens gekennzeichnet werden.

17 - Dieser Wort-Marker wird nicht nur für die lexikalischen Hauptkategorien angenommen, sondern auch $2 . B$. für das Pronomen; vgl. dazu $\$ 6.0$.

18 - Zu den Flexionsregeln im Polnischen vgl. Pohl 1983a und $1983 b$.

19 - In Chomsky $(1965,85)$ werden diese Merkmale im Hinblick auf die selektionsregeln aufgestellt; hier stehen: APPELLATIVUM für COMMON: INDIVIDUATIVUM für COUNT; BELEBT für ANIMATE u.s.w. entsprechend Chomsky $(1965,82)$. Auf diese Merkmale kann hier nicht verzichtet werden, da nun mal die slavischen Sprachen und bes. das Polnische - bereits unabhängig von Chomsky und Katz - diese Merkmale erfordern.

20 - Vgl. die eingehende Beschreibung der Morphonologie des NOM und GEN im Polnischen und im Russischen in Pohl 1983a und 1983b. 21 - Zur Anwendung der X-Bar-Strukturen von Jackendoff 1977 siehe: Pohl 1983b. Die Schwierigkeiten mit der Einsetzung der Kasus entstehen nicht so sehr in der subjekt-als in der objekt-Phrase, wo es $\mathrm{zu}$ Uberschneidungen der Verzweigungen kommen kann, sobald GEN, DAT, AKK und INS zugleich eingesetzt werden müssen. Eine bessere Lösung bietet Chomsky 1981; vgl. Pohl 1983c. 
22 - Lyons $(1977,411)$ weist darauf hin, daB sich Katz \& Fodor auf die Theorie Hjelmslevs stützen.

23 - Als Beispiel führt Weinreich die Subkategorisierung des transitiven und des intransitiven verbs an, indem er bereits die Subkategorisierung als lexikalische Einsetzungsregeln notiert. zur Subkategorisierung des polnischen Verbs, bzw. zu seiner Valenz siehe: Polanski, K. /red./ 1980 / 1984, Słownik syntaktyczno-generatywny czasowników polskich, $t$. I-II, Wrocław et al.

24 - So unterscheidet er u.v.a. z.B. zwischen einer Modalisienung, als Anweisung, die semantische Einheit nicht wörtlich zu fassen, oder einer Begrenzung, als Einschränkung der Klasse von Referenten eines zeichens, oder aber einer Einnistung, z.B. als Verbindung des Verbs mit dem $z u$ ihm passenden Nomen in objektstellung. 25 - Proposition im Sinne des semantischen Kerns eines Satzes neben den modalen Relationen; Argument im Sinne einer Subjekt-Variablen bzw. im Sinne eines Subjekt-Von; Prädikat im Sinne Prädikat-Von; Präsupposition im Sinne von Satzvorstellungen; Topic als Element der Tiefenstruktur, die das Thema des zu erzeugenden Satzes bestimmt.

26 - Zum Vergleich beider Semantik-Auffassungen siehe: Lyons $(1977, \S 10.5)$.

27 - Im Rahmen der hier $z u$ beschreibenden Nominalisierung im Polnischen wird jedoch die Feststellung gemacht, daB Distinktoren, sofern man sie im Katzschen Sinne als Kennzeichnung des Einmaligen an der Bedeutung eines Worts aufaBt, in den Wortbildungsregeln keine entscheidende Rolle spielen, weil die Bedingungen für die Affigierung ohne eine Bestimmung der Einmaligkeit an der Bedeutung eines Worts auskommen. Allerdings gibt es Merkmale, wie z.B. FORTBEWEGUNGSMITTEL, die vielleicht in einem semantischen Merkmalsystem des Polnischen, über das wir nicht verfügen, in Opposition zu einem anderen Merkmal gebracht werden könnte; vorerst hat dieses Merkmal die Funktion eines "Distinktors", wenngleich nicht für ein Wort allein, sondern für eine ganze Wortgruppe, die ein bestimmtes Affix gemeinsam hat. Die negative Markierung dieses Merkmals impliziert alles, was nicht zu den 'Fortbewegungsmitteln' gehört, jedoch ebenfalls durch 'Instrumentalität' gekennzeichnet ist. 
28 - Die Quantoren haben Elementaraussagen zur Folge, die auf Grund der Gegenstandsvariablen getroffen werden. Dabei sind die Gegenstandsvariablen durch Junktoren, aber auch durch Quantoren mit einander verbunden. Folglich bindet ein Quantor Q sowohl Junktoren als auch Quantoren.

29 - Vgl. dazu bes. die Arbeiten von Anna Wierzbicka und Andrzej Bogusławski.

30 - Ich gehe hier von der Feststellung aus, daß die $\bar{x}-$ Syntax - wenngleich nicht in der Version von Jackendoff 1977 - eine beschreibungsadăquate Methode auch für die syntax des polnischen darstellt, wenn sie als Merkmal-syntax verstanden wird.

31 - Die Unterscheidung der "Lexikalisten" zwischen Regieren und Präzedenz wird von den "Transformationalisten" durch die Unterscheidung zwischen relationalen und nicht relationalen Elementen - vgl. u.a. Kilby 1977 -, die kategorial nicht differenziert sind und Bachs $1968 \mathrm{klassen}$ von Contentives entsprechen, ersetzt. 32 - Hier ist auch der Grund dafür zu sehen, daß Wortbildungstheorien, wie z.B. Wurzel 1970 oder Laskowski 1981 - Transformationen in die Wortbildung einbeziehen.

33 - Zur Einbeziehung dieser Beispiele in die $\bar{x}$-Syntax sowie zu ihrer Bildung siehe: Jackendoff 1977.

34 - Zum Possessivus im Polnischen siehe: Pohl 1983c und 1983d. 35 - Im Polnischen gibt es bekanntlich keinen Artikel, aber wohlweislich eine Unterscheidung zwischen [ \pm BESTIMMT], die u.a. sowohl durch ein Demonstrativpronomen als auch durch die Anwendung eines entsprechenden Kasus ausgedrückt werden kann. Was die Kennzeichnung durch [ \pm BESTIMMT] durch die kasus betrifft, so gilt z.B. der GEN PARTITIVUS für [- BESTIMMT] z.B. in kupizem mqki 'ich kaufte Mehl' vs. AKK kupizem mqke 'ich kaufte das Mehl' oder on jest aktor (NOM) 'er ist ein Schauspieler' vs. on jest aktorem (INS) 'er ist Schauspieler'; siehe dazu: Sadzifiski, R. 1981, Der unbestimmte Artikel in Deutschen und dessen Äquivalenzstruktur im Polnischen. In: A. Szulc (Hrsg.) 1981, studien zum polnisch-deutschen Sprachvergleich. Warszawa-Kraków. S. $23-29$. 36 - Die jeweilige Repräsentation von SPEC ist auch für das Polnische gültig; vgl. Pohl 1983b. 
37 - zitat (S. 334): "... the number of symbols in a rule is inversely related to the degree of linguistically significant generalization achieved in the rule."

38 - Laskowski belegt diese Behauptung durch wörter, die in das Polnische ohne die dazugehörenden Wortbildungsregeln aus anderen Sprachen entlehnt werden; so z.B. Marks - marksista 'Marx-Marxist', wobei dem entlehnten Wort das Suffix-ist-hinzugefügt wird; dieses mit der Bedeutung 'Anhänger einer Theorie (Ideologie)', die durch den Träger des Namens vertreten wird (S.111). 39 - Die Notation der morphonemischen Repräsentation der Suffixe entspricht der von Laskowski 1975 und von Gussman 1978.

40 - Das Merkmal COMP wird hier - wie bemerkt - nach Chomsky 1970 rechts von $\mathrm{N}$ gesetzt. Innerhalb der S-Basis entspricht diese Position dem WH-MOVEmENT; vgl. dazu Radford $(1981,242 \mathrm{ff}$.).

41 - Radford $(1981,323)$ schreibt: "NP and S-bar are absolute barriers to government (i.e. one category cannot govern another across an intervening NP or $S$-bar boundary).

42 - Entsprechend der Definition von Radford (1981,319):

" $X$ governs $Y$ just in case

(i) $X$ is a governing node c-commanding $Y$

(ii) there is on other governing node $z$ such that:

(a) X c-commands $\mathrm{Z}$

\& (b) Z c-comnands $Y$

\& (c) 2 does not c-commands $X$ "

kann festgestellt werden, daB NP sowohl in $(49(i))$ als auch in (49(ii)) $\bar{N}$ c-kommandiert; $\bar{N}$ dagegen c-kommandiert in (49(i)) $\bar{N}$, in $(49(11))$ hingegen $\overline{\bar{A}}$.

43 - Dieses Problem erfordert eine gesonderte Untersuchung.

44 - Das es sich bei den sog. Pronomina um Adjektiva handelt, dies bezulglich ihrer stellung in der S-Basis, wird eingehend in Pohl 1983d gezeigt.

45 - Zur Bildung der Possessiv-Adjektiva im Russischen vgl. Pohl 1984.

46 - Geht man von einer Wortbildung aus, die auf Merkmalen beruht, so ist die Derivation von Präpositionen - vgl. die Merkmale für die Prăpositionen bei Weinreich 1966 - denkbar, wobei die 
morphosyntaktische struktur auch hier ihre Anwendung finden könnte.

47 - Zum NOM PL im Polnischen und im Russischen siehe: Pohl 1983a. 48 - Zu den Kasus in der generativen Grammatik des Polnischen und des Russischen siehe: Pohl 1983c.

49 - Zur generativen Beschreibung der Kasus im Deutschen siehe: Bierwisch 1967

50 - Siehe dazu u.a.: Brekle $(1972,27)$.

51 - Zum TF im Russsischen siehe: Isačenko 1969.

52 - Zur semnatischen Funktion des Evaluators siehe: Stankiewicz, E. 1961, Grammatical Neutralization in Slavic Expressive Forms. In: Word 17/2, S. 128 - 145; auBerdem: Pohl 1980b.

53 - Zur ausführlichen Diskussion der Morphonologie des Polnischen siehe: Laskowski 1975, Paulsson 1975 und Gussman 1978. 54 - Mit der Annahme eines Idealfalls tun sich die Autoren des KAR schwer. Sie lehnen die bisherigen Arbeiten - KAR ist 1977 erschienen - zur polnischen Phonetik ab (!) mit der Begründung, daB all diese Arbeiten den heutigen Erfordernissen weder in ihrer Reichweite noch in ihrer Methode und Darstellung genügetun (S. VII). Anschließend nehmen sie einen durchschnittlichen, gebildeten Benutzer des Polnischen - im Sinne von literaturnyj jazyk - an, ohne diesen näher zu erläutern, und gleichzeitig den gebildeten Polen (S. XVII). Schlieblich wird eine soziale Dreiteilung der polnischen Sprache vorgenommen, namlich in einen Kulturdialekt, gesprochen von der sozialen oberschicht, einen Stadtdialekt, gesprochen von ungebildeten Städtern, und einen volksdialekt, gesprochen von der sozialen Unterschicht. Der "Kulturdialekt" wird unterteilt in: Literaturprache = Schriftsprache; Feierlichen Kulturdialekt; Gepflegter umgangskulturdialekt sowie legerer umgangskulturdialekt (S. XXII). Bei diesen Unterteilungen bleibt jedoch die Heterogenität der MaBstäbe unbeachtet; soziale stellung und Bildung im heutigen Polen als direkt proportionale GröBen aufzufassen hat weder etwas mit der polnischen Sprache noch etwas mit der polnischen wirklichkeit zu tun.

55 - Es wäre auch nicht im Sinne meiner Ausführungen, die Spra- 
che der polnischen Medien als Stütze zu zitieren, denn diese wird häufig als Verstümmelung der polszczyzna kulturalna kritisiert.

56 - Szober $(1962,18)$ fügt dem $Z$ die Eigenschaften: apikal-dental und oral als einzige Artikulationsmöglichkeit zu. KAR geht dagegen vom Verschwinden dieses $Z$ aus und postuliert allein [w], also bilabial-rund. Wierzchowska $(1980,118)$ charakterisiert $Z$ als einen lateralen, koronal-alveolaren Laut.

57 - Zur Assimilation im Polnischen siehe bes.: Paulsson 1979.

58 - Die Annahme des flüchtigen Vokals in diesem $K$ ist hypothetisch; eine gesonderte Untersuchung der Adjektivierung im Polnischen müBte zeigen, wann und ob überhaupt die Annahme eines flüchtigen Vokals in dieser Position sinnvoll wäre.

59 - Welches dieser beiden systeme brauchbarer ist, entscheidet keineswegs die Tatsache, daß die Wortbildung in der Eweiterten Standard-Theorie angesiedelt ist. Beide systeme definieren die lexikalischen Kategorien auf ihre Weise mittels zuordnung von Kategorien, die universal sind. Während schmid auf symmetrie wert legt, betonen die Generativisten die Okonomie, indem sie auf die Redundanzen verzichten, diese aber in zusatzregeln ausfüllen, wie z.B. bei der Definition von PRO; vgl. dazu Radford $(1981,111)$.

60 - Zum NOM PL siehe: Pohl 1983a.

61 - Bei der Berücksichtigung der 'Dimensionalität' stütze ich mich auf Greimas 1966, der spatialité in dimensionalité und non-dimensionalité unterteilt. Inwiefern die 'Dimensionalität' neben der 'Räumlichkeit' im Polnischen eine innersprachliche, d.h. eine mit der Affigierung zusammenhängende Funktion hat, könnte in einer gesonderten Untersuchung der 'räumlichen' Nomina des Polnischen geklärt werden.

62 - Diese Merkmale sind in erster Linie als Ergebnis meiner Beschäftigung mit dem Wortkorpus hauptsächlich in DOR zu sehen, angeregt jedoch durch die in $\S 4.1$ referierte semantische Komponente: diese Merkmale haben provisorischen Charakter. Solch eine Lösung scheint mir insofern sinnvoll zu sein, als es hier nicht um die Bestimmung von Universalien, sondern um die Bestimmung von Bedingungen für die Nominalisierung im Polnischen geht. 
63 - Lubaszewski $(1982,16)$ ordnet nämlich jeder Wurzel des zu bildenden Verbs ein Kategorien- oder ein Interjektionsmerkmal zu. Die R-Repräsentation [- prac +] erhielte demnach das Kategorienmerkmal $\mathbf{N}$.

64 - Fine umfassende Untersuchung der Wurzelnomina könnte die morphonemischen Repräsentationen in Verbindung mit entsprechenden Merkmalen bringen und diese als Bedingungen für die Affigierung einsetzen lassen; darunter wären auch die Genusmerkmale. Ob es sich dabei um Merkmale auBersprachlicher sinnbezirke handeln müBte, bleibt dahingestellt; möglich wäre, daß hier bestimmte morphonemische Repräsentationen allein und ausschlieblich über die Affigierung mit Hilfe des "Null"-Klassifikators entscheiden. 65 - Es gehört zu den Aufgaben der Lehnwortforschung, genauer, zu den Aufgaben der Lehnwortphonologie, im Falle dieser wörter zu bestimmen, unter welchen Bedingungen bestimnte deutsche Phonemverbindungen ins Polnische integriert werden, so auch kürsenaere zu kub́nierz oder soldenaere zu ioznierz, aber sold zu iozd. 66 - Bei der Bildung von taksiarz wird 8 palatalisiert; denn es wird artikuliert [taksi], nicht *[tak'si], aber [takśaś].

67 - Dabei handelt es sich bei stolarz mit sicherheit um eine Lehnbildung aus dem Deutschen, nämlich: Tisch-l-er $\rightarrow$ stol-arz: dies in dem Sinne, daB - im Gegensatz zum Lehnwort - nicht das ganze Wort übernommen wird, sondern das der polnischen Phonologie entsprechend angepaßte suffix, während der wortstamm ins Polnische übersetzt wird.

68 - Es wäre möglich, für die Nomina bibliotekarz, aptekarz als Bedingung das Merkmal der 'Räumlichkeit' anzunehmen, dem der Stammauslaut zugeordnet werden kann; doch müBte dann sekretarz ausgeschlossen werden. Also wird der Stammauslaut als Bedingung für die Affigierung vorgezogen.

69 - Für sekretarz gălte demnach sekretariat: doch dies bedeutete, für dieses wort eine gesonderte Regel aufzustellen, un gleich zwei Affixe zu tilgen, nämlich -ari- und -at. Solch eine Lösung wäre wenig sinnvoll, wenn man solche wörter, wie $2 . B$. komisariat 'Kommissariat' mitberücksichtigt und feststellen mu, daB es die 'Räumlichkeit' ist, die nach dem 'Berufsträger' - hier komisarz 'Kommissar' - benannt ist und nicht umgekehrt. Also liegt auch 
in sekretarz ein Lehnwort vor, dessen Morphologie mitübernommen worden ist; dies gilt selbstverständlich auch für das Nomen $8 e-$ kret 'Geheimnis': vgl. lat. sekretarius, mhd. Sekretar, aber frz. secrétaire, ital. segretario.

70 - Das Merkmal [+ INSTRUMENTALITÁT] muB für die Einbeziehung der Kasus entsprechend differenziert werden. Im GEN SG der Maskulina z.B. wird zwischen 'Werkzeugen' und anderen 'Instrumen-

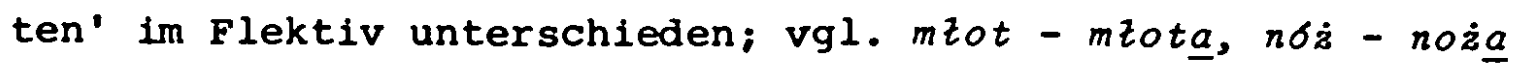
'Hammer, Messer' aber fagot - fagotú 'Fagott': siehe dazu: Pohl $1983 b$.

71 - Es muB zwischen dem Klassifikator - $i k$ und dem Evaluator - $i k$ bzw. -yk unterschieden werden; vgl. dziennik 'Journal' aber koszyk 'Körbchen'.

72 - Zu obdach vgl. SW. Es gibt zwar das polnische obdaszenie 'Uberdachung', auch das Diminutiv obdaszek 'Dächlein' neben dach 'Dach', aber kein Verb *obdaszyc oder * obdachowac, die ob- als polnisches Präfix einstufen ließen; daher wird obdasznica als denominales Nomen verstanden. (Auch in diesem Fall wird deutlich, das auf die Einbeziehung der Wurzelnomina in die Wortbildung nicht verzichtet werden kann; es sei man eliminiert auch Lehnwortstäme, in diesem Fall das deutsche Obdach, das allerdings nach Grimm hauptsächlich 'Heim, Geborgenheit' bedeutet.)

73 - Zu den Wurzelnomina mit dem Suffix -ec gehören neben samiec auch ojciec 'Vater', chzopiec 'Junge'; denn wie soll man vom Sprecher des Gegenwartspolnisch erwarten, daB er chzopiec von chtop 'Kerl, Mann, Bauer' bildet.

74 - Es wăre eine Aufgabe für die diachronische Wortbildung, diese wörter im Hinblick auf eine deadjektivische Bildung zu untersuchen; es gibt nämlich im Altpolnischen noch goscin-i neben goscin-y 'den Gast betreffend', desgleichen noch im SW sierocin 'Waisen-'. Nach SkA ist dziedziniec von *dèti 'Kinder' gebildet und entspricht der historischen Bedeutung 'Platz für Kinder'. 75 - Fegert \& Rodekuhr 1981 nehmen einen sog. Unverträglichkeitsvokal zwischen "Stern, Komma" und dem Suffix-STV-. Da es sich bei dieser Arbeit um den Versuch einer konfrontativen Beschreibung des russischen und des polnischen suffixes -STV- handelt, ist die Annahme solch eines Vokalsegments, das im Russischen un- 
ter bestimmten Bedingungen als Vokal realisiert wird, sinnvoll. Im Polnischen scheint diese stelle in nur wenigen wörtern als Vokal realisiert $z u$ werden, so z.B. in jestestwo, nicestwo 'Sein, Nichts'.

76 - In DOR wird robotnik mit 'Vertragsarbeiter' notiert, der 'körperliche Arbeiten auf Kosten und Risiko des Arbeitgebers durchfuhrt'. Als Konnotat gelten: 'engagieren, mieten, beschäftigen'; hinzu kommt robotnik pansaczyzniany 'Leibeigener'.

77 - Die Vokal-Null-Alternation wird z.B. wegen der Kurzform winien statt winny von wina 'schuldig - Schuld' angenommen. 78 - Eine genaue Kennzeichnung des 'Grades' an 'Weisheit', 'Geiz' u.s.w. für die Ausdrücke medrzec, skqpiec mübte eine semantische Untersuchung des Polnischen zeigen.

79 - Die hier notierten K-Repräsentationen stellen eine Auswahl dar, erheben daher keinen Anspruch auf eine vollständige Verbklassifikation des Polnischen. Zur generativen Beschreibung des polnischen Verbs siehe: Paulsson 1974 und Lubaszewski 1982. 80 - Mit Ausnahme des GEN und des AKK PL. Die Nomina bZuźnierca, szyderca, morderca 'Lästerer, Spötter, Mörder' können nicht als deverbal klassifiziert werden. Mit sicherheit gehen sie auf die älteren bluźnierz (STP, Erstbeleg ca. 1500), szyderz (LIN, Erstbeleg 1595), morderz (Cnapius) zurück. Wollte man sie dennoch als deverbal im Gegenwartspolnisch sehen, so mübte ein TF -erangenommen werden, und im Falle von morderca mübte der verbale klassifikator -owa- getilgt werden, denn das verb heibt mordowac 'morden'. Da es sich jedoch um nur drei wörter handelt, wird auf das Aufstellen einer Regel verzichtet.

81 - Das Nomen spozdzielnia 'Kooperativ' past hier nicht hinein; entsprechend DOR wird es vom Verb spozdziazac 'mitwirken' gebildet. Der stammauslaut enthält eine a/e-Alternation, die mit dem TF - aZ- offensichtlich eine Uberlappung zur Folge hat. Um diese zu vermeiden, wird kein TF hinzugefügt. (Erstbelege in DOR alle nach 1945; wăhrend Wielka Ilustrowana Encyklopedia Powszechna, Bd. XVI /1931-32/, Gutenberg Kraków, bereits auf den Beleg in Ustawa Społdzielcza vom 29.10.1920 hinweist.)

82 - Um das Interfix, das zwei wortstäme in Kompositum mit einander verbindet, in die morphosyntaktische struktur (61) einzu- 
beziehen, bedarf es allein der Erweiterung des Wortbildungsteils um die bereits gekennzeichneten Grenzen sowie des Einschubs des Vokals -o-, um zwei durch WT gekennzeichnete Klammern dem Fle$x$ ionsteil voranzustellen.

83 - Das Nomen ryk kann im Deutschen nur durch 'Gebrüll' wiedergegeben werden; es hat aber auch die zusätzliche Bedeutung des einmaligen 'Schreis'. Der Schrei ist auch im Deutschen als 'Resultat' des Verbs schreien $z u$ werten.

84 - Die Nomina prośba, siejba gehören zu den noch vor dem 16. Jahrhundert - so in STP - belegten wörtern.

85-Eine Entscheidung darüber, welche Verben maskuline und welche feminine 'Resultate' ergeben, müßte eine gesonderte Untersuchung des polnischen Verbs fällen.

86 - Zur resultativen Aktionsart im Russischen vgl. Isačenko $(1975, \S 216$, D), im Polnischen Szober $(1962, \S 251)$.

87 - Vgl. dazu das Merkmal [PARS] in Pohl $1983 \mathrm{c}$.

88 - Diese Feststellung kann bei der Sichtung der Wortkorpora in Klemensiewiczówna 1951 und in kurzowa 1976 gemacht werden. 89 - Um die "Null"-Klassifikatoren in die genusorientierte Nominalisierung einbeziehen zu können, müßte vielleicht doch von einer strikten Trennung zwischen Wortbildung und Wortbeugung abgesehen und die NOM-SG-Form samt Flektiv in die wortbildung einbezogen werden; zumal man davon ausgehen kann, daß der sprecher des Polnischen ganz offensichtlich das Genus mit dem Flektiv assozilert.

90 - Mit Sicherheit ist es in der studentensprache auch der Mechanismus der Analogie, der die Bildung solcher Nomina zur Folge hat, so z.B. waryniec in Analogie zu zwierzyniec ' $z 00$ '; doch gerade diese Analogie zeigt, daß die inhärenten Merkmale den Affixen des "Vorbildworts" und nicht der potentiellen Wortverbindung ogrod zwierzyny 'Garten des Tiers' zugeordnet werden. Zur Studentensprache siehe: L. Kaczmarek \& T. Skubalanka \& S. Grabias, Słownik gwary studenckiej. Zakład Narodowy im. Ossolinskich - Wydawnictwo 1974. 


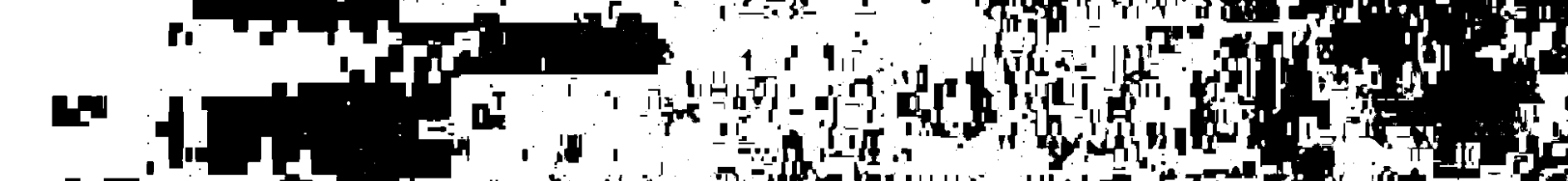

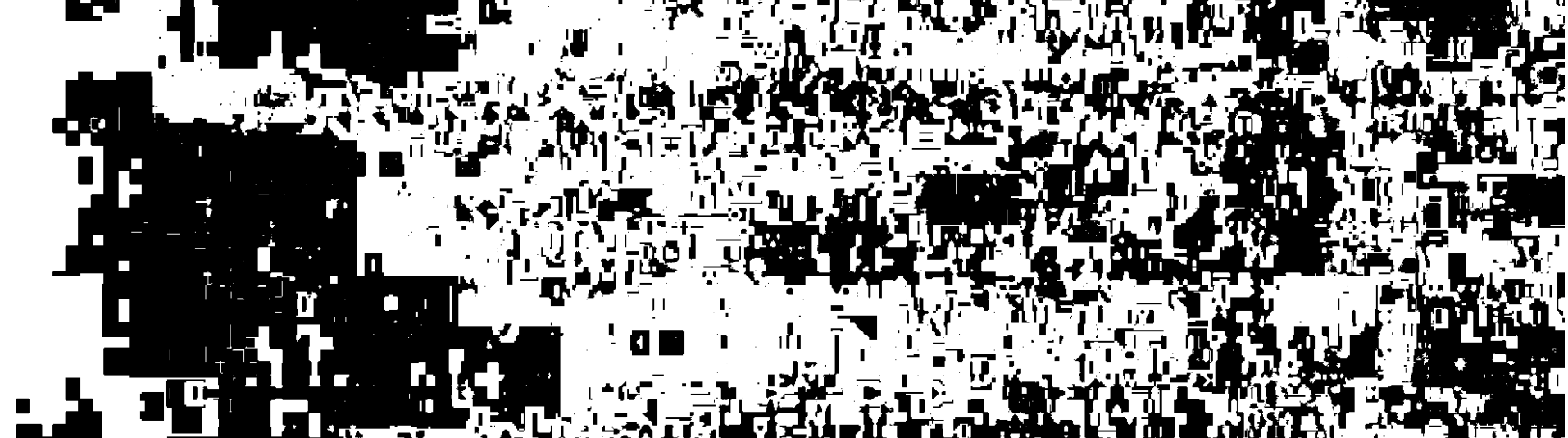

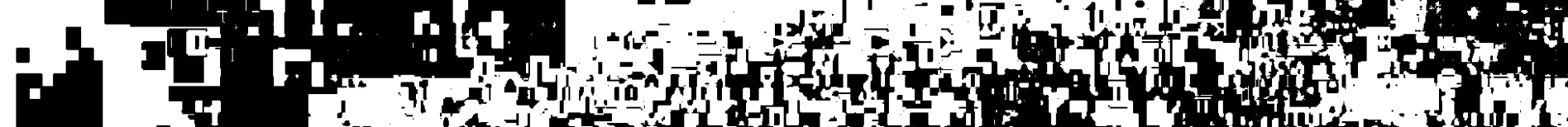

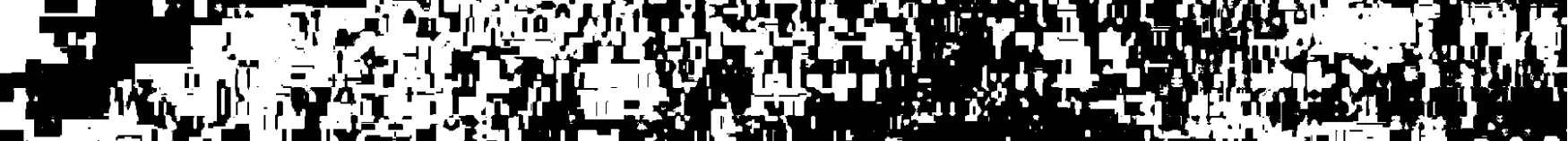

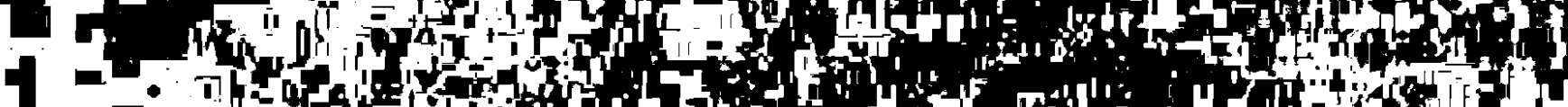

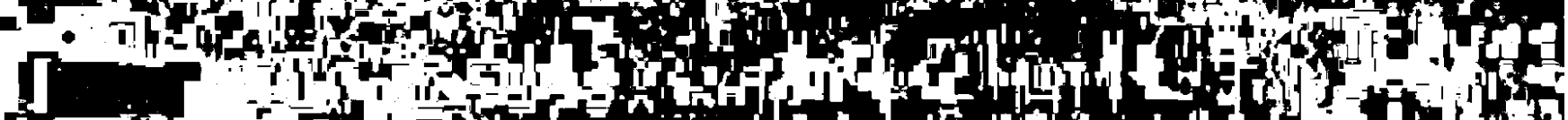

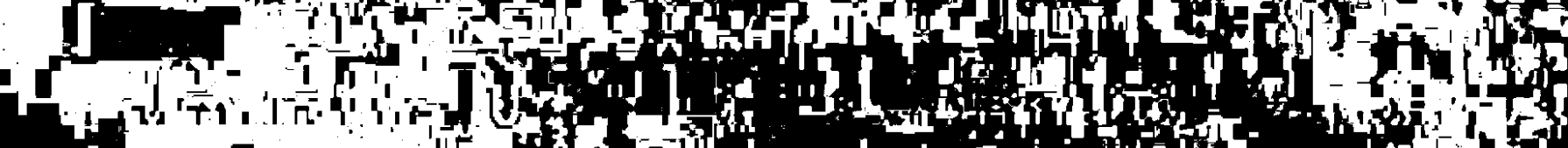
Dat

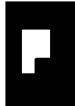
1 ti

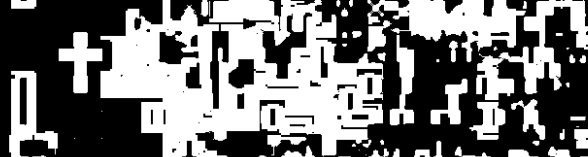

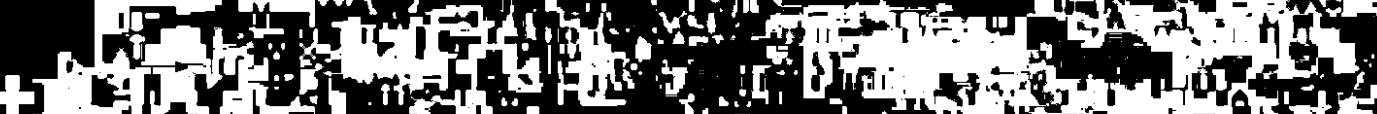

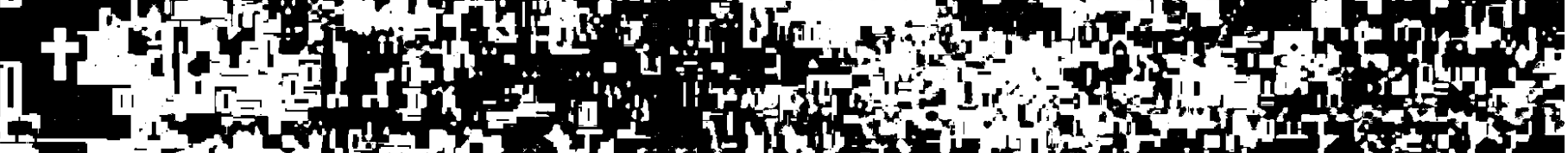
-

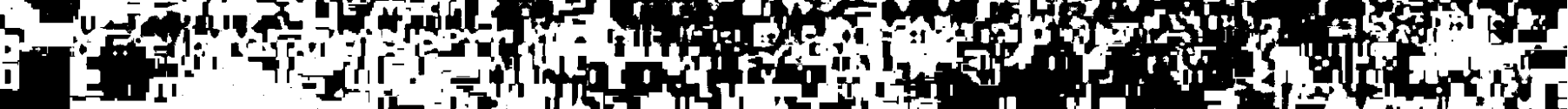

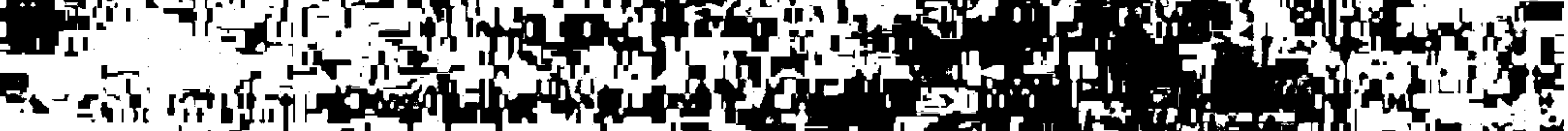
0 -

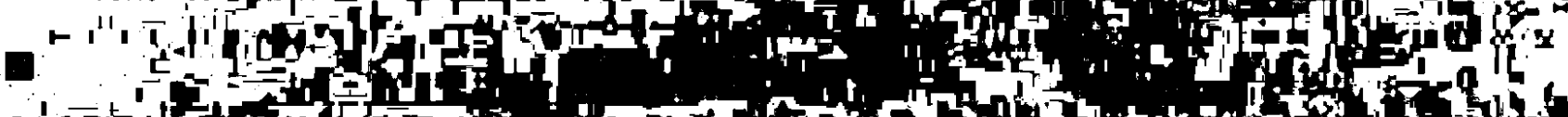

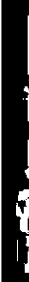

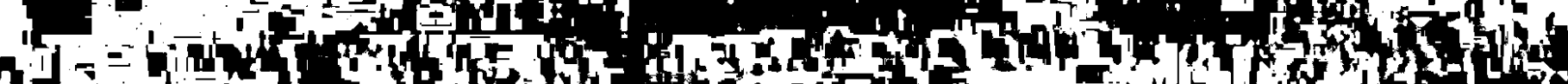

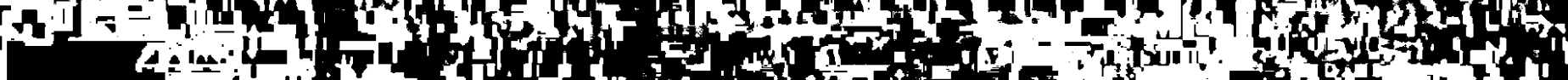

7

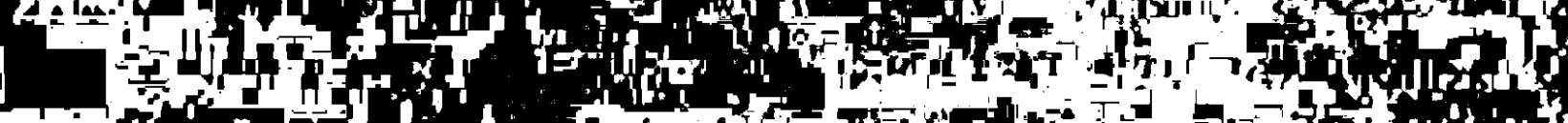

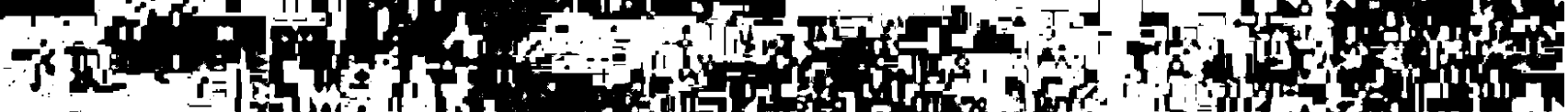

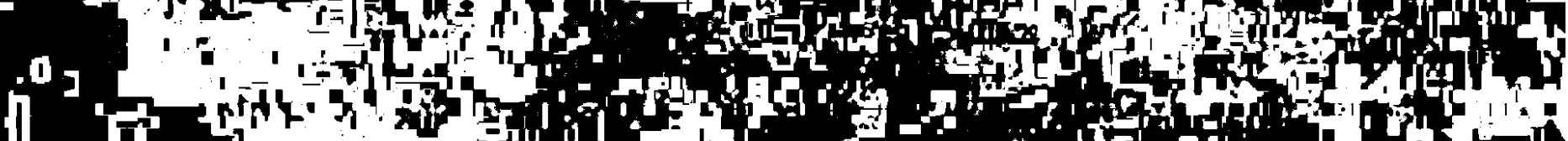
1 in

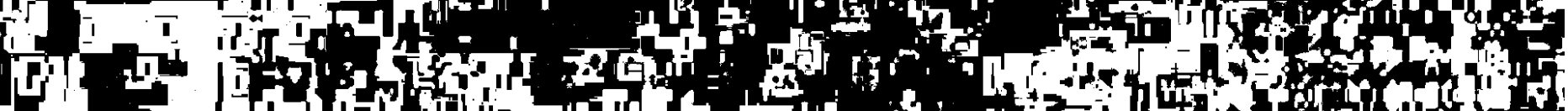

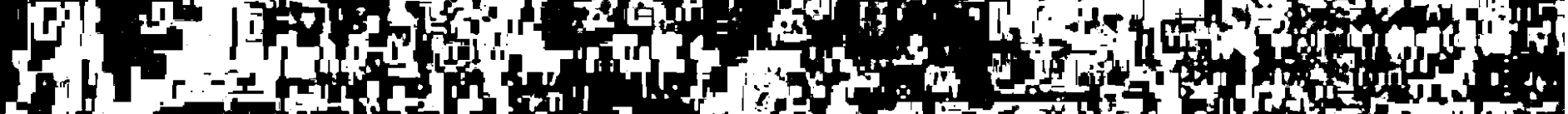
$A=1,4 w^{2}$

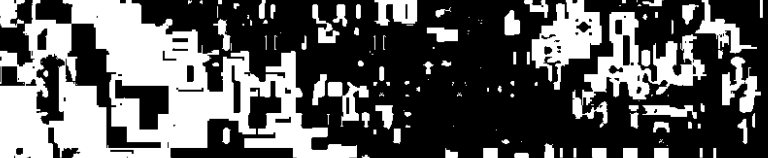

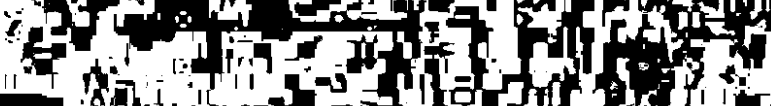
- mover 1)

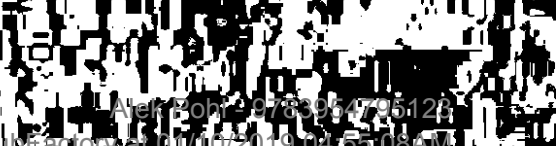




\section{LITERATUR}

Aronoff, M. 1976, Word-Formation in Generative Grammar. In: Linguistic Inquiry Monograph 1, Cambridge Mass.

Bach, E. 1968, Nouns and Noun Phrases. In: E. Bach \& R. T. Harms (Hrsg.) Universals in Linguistic Theory. New York 1968, S. $91-122$.

Baudouin de Courtenay, J. N. 1888, o zadaniach jezykoznawstwa. In: Doroszewski 1974, S. $24-49(176-201)$.

Baudouin de Courtenay, J. N. 1895, Opyt teorii fonetičeskich alternacij, Glava iz psichofonetiki. In: Grigor'ev \& Leont'ev 1963, Bd 1, S. 265 - 347.

Baudouin de Courtenay, J. N. 1908, o svjazi fonetičeskich predstavlenij s predstavlenijami morfologičeskimi i semasiologičeskimi. In: Grigor'ev \& Leont'ev 1963, Bd. 2, S. $163-174$. Benni, T. 1959, Fonetyka opisowa jezyka polskiego. Wrocław. Bierwisch, M. 1965, Eine Hierarchie syntaktisch-semantischer Merkmale. In: Syntaktische strukturen, studia Grammatica V, S. $29-86$.

Bierwisch, M. 1967, Syntactic Features in Morphology: General Problems of the so-called Pronominal Inflection in German. In: To Honor Roman Jakobson, Essays on the occasion of his seventieth birthday. The Hague. S. $239-270$.

Botha, R. P. 1968, The Function of the Lexicon in Transformational Generative Grammar. The Hague - Paris.

Botha, R. P. 1974, Problematic Aspects of the Exception Filter in Professor Halle's "Morphology". Indiana University Club (Typoskript).

Brekle, H. E. 1972, Semantik. Eine Einfuhrung in die sprachwissenschaftliche Bedeutungslehre. München.

Brodowska-Honowska, M. 1967, Zarys klasyfikacji polskich derywatow. Wrockaw et al.

Chomsky, N. 1965, Aspects of the Theory of Syntax. Cambridge Mass. ${ }^{8} 1972$.

Chomsky, N. \& Halle, M. 1968, The Sound Pattern of English. New York et al. 
Chomsky, N. 1969, Deep structure, Surface structure and semantic Interpretation. In: Chomsky 1972, S. $62-119$.

Chomsky, N. 1970, Remarks on Nominalization. In: Chomsky 1972, S. $11-61$.

Chomsky, N. 1972, Studies on Semantics in Generative Grammar. The Hague.

Chomsky, N. 1975 = Chomsky 1977 Reflexionen uber Sprache. Frankfurt a. M.

Chomsky, N. 1981 Lectures on Government and Binding. Dordrecht - Cinnaminson.

Coseriu, E. 1970, Einführung in die strukturelle Betrachtung des Wortschatzes. Tübingen.

Coseriu, E. 1973, Probleme der strukturellen Semantik. (Vorlesung gehalten im Wintersemester $1965 / 66$ an der Universität Tüingen. Autorisierte und bearbeitete Nachschrift von Dieter Kastovsky) Tübingen.

Dokulil, M. 1962, Tvor̆eni slov v češtinè. C. 1: Teorie odvozovánî slov. Praha.

Doroszewski, W. et al. (Hrsg.) 1974, J. N. Baudouin de Courtenay- Dzieła wybrane. T. 1, Warszawa.

Dunaj, B. 1979, Zarys morfonologii wspołczesnej polszczyzny. In: Skrypty uczelniane Uniwersytetu Jagiellonskiego Nr. 327, Krakow.

Fegert, H. \& Rodekuhr, G. 1981, Die Morphonologie der Neutra auf -STV- im Polnischen und Russischen. Ein Beitrag zur vergleichenden Morphonologie der slavischen Sprachen. In: A. de Vincenz (Hrsg.) Slavistische Linguistik 4, Frankfurt a. M. - Bern.

Fokker, A. A. 1966, Nouns from Verbs. A Contribution to the study of Present Day Polish wort Formation. Amsterdam.

Gardner, Th. 1975, Einführung in die moderne englische Phonologie. Heidelberg.

Greimas, A. J. 1966, Semantique structurale. Recherche de méthode. Paris. 
Grigor'ev, V. P. \& Leont'ev, A. A. (Hrsg.) 1963, Boduen de Kurtene. Izbrannyje trudy po obščemu jazykoznaniju. T. 1-2. Moskva.

Grzegorczykowa, R. ${ }^{3} 1979$, Zarys słowotworstwa polskiego. Słowotworstwo opisowe. Warszawa.

Grzegorczykowa, M. \& Puzynina, J. 1979, Słowotwórstwo współczesnego jezyka polskiego. Rzeczowniki sufiksalne rodzime. Warszawa.

Gussman, E. 1978, Contrastive Polish-English Consonantal Phonology. Warszawa.

Gussman, E. 1980, Introduction to phonological Analysis. Warszawa.

Halle, M. 1959, The Sound Pattern of Russian. The Hague - Paris. Halle, M. 1973, Prolegomena to a Theory of Word Formation. In: Linguistic Inquiry IV, S. 3 - 16 .

Heidolph, K. E. \& Fläming, W. \& Motsch, W. 1981, Grundzüge einer deutschen Grammatik. Berlin.

Hjelmslev, L. 1953, Prolegomena to a Theory of Language. Bloomington Ind. (Dänisch 1943).

Honowska, M. 1979, Ewolucja metod polskiego słowotwórstwa synchronicznego (w dziesiecioleciu 1967 - 1977). Wroclaw et al.

Ineichen, G. 1979, Allgemeine Sprachtypologie. Ansätze und Methoden. Darmstadt.

Isačenko, A. V. 1961, o grammatičeskom značenii. In: Voprosy jazykoznanija 10, s. $28-43$.

Isačenko, A. V. 1969, Morpheme Classes, Deep Structure, and the Russian Indeclinables. In: IJSLP 12 (1969), S. 48 - 72.

Isačenko, A. V. 1974, Morphonologische Motivierung phonologischer Merkmale. Zur Morphonologie der sog. femininen i-Stämme im Russischen. In: Phonologica II, S. 335 - 352 .

Isačenko, A. V. ${ }^{3} 1975$, Die russische sprache der Gegenwart. Formenlehre. München.

Jackendoff, R. S. 1975, Morphological and Semantic Regularities in the Lexicon. In: Language LI, S. $639-671$. 
Jackendoff, R. S. 1977, $\bar{x}$ - Syntax: A Study of Phrase Structure. Cambridge Mass. - London.

Jacobs, R. \& Rosenbaum, P. S. (Hrsg.) 1970, Readings in Englissh Transformational Grammar. Waltham Mass.

Jakobson, R. 1932, Zur Struktur des russischen Verbums. In: Selected Writings II. The Hague - Paris 1971. S. 3 - 15.

Jakobson, R. 1939, Das Nullzeichen. In: Selected Writings II. The Hague - Paris. S. $220-222$.

Jakobson, R. 1944, Russian Conjugation. In: Word 4, 1948. S. 155-167 .

Katz, J. J. \& Fodor, J. A. 1963, The Structure of a Semantic Theory. In: Language 39, S. $170-210$.

Katz, J. J. \& Postal, P. M. 1964, An Integrated Theory of Linguistic Descriptions. Cambridge Mass.

Katz, J. J. 1972, Semantic Theory. New York.

Kilby, D. A. 1977, Deep and Superficial Cases in Russian. In: o. Horbatsch \& G. Freidhof (Hrsg.) Specimina Philologiae sl.avicae, Bd. 14. Frankfurt a. M.

Klemensiewiczóna, I. 1951, Wyrazy złożone nowszej polszczyzny kulturalnej. Kraków.

Koschmieder, E. 1977, Phonationslehre des Polnischen. München.

Kowalik, K. 1977, Budowa morfologiczna przymiotnikow polskich. Wrocław.

Kurzowa, z. 1976, złożenia imienne we wspołczesnym języku polskim. Warszawa - kraków.

Lakoff, G. 1971, On Generative Semantics. In: D. D. Steinberg \& I. A. Jakobovits (Hrsg.) Semantics. London - New York 1971. S. $232-296$.

Laskowski, R. 1971, Uwagi o miejscu słowotworstwa w gramatyce generatywnej. In: Koferencja Naukowa "System morfologiczny i syntaktyczny wspołczesnego języka polskiego" zawoja 16. - 18. XII 1971. Kraków.

Laskowski, R. 1972, Polnische Grammatik. Warszawa - Leipzig. 
Laskowski, R. 1975, Studia nad morfonologią współczesnego języka polskiego. Wrocław et al.

Laskowski, R. 1977, Morfologia w gramatyce transformacyjno-generatywnej. W poszukiwaniu modelu opisu. In: S. Urbanczyk (Hrsg.) Studia gramatyczne I, S. 103 - 133.

Laskowski, R. 1981, Derywacja słowotworcza. In: J. Bartminski (Hrsg.), Pojęcie derywacji w lingwistyce. Lublin. S. 107 $-126$.

Lubaszewski, W. 1982, Struktura morfemowa polskiego czasownika. Próba opisu generatywnego. Wrocław et al.

Lyons, J. 1968, Introduction to Theoretical Linguistics. London- New York.

Lyons, J. 1977, Semantics I \& II. Cambridge et al.

McCawley, J. D. 1968, The Role of Semantics in a Grammar. In:

E. Bach \& R. T. Harms (Hrsg.) Universals in Linguistic Theory. New York 1968. S. $124-169$.

McCawley, J. D. 1969, where do Noun Phrases come from? In: Jacobs \& Rosenbaum 1970, S. 166 - 183.

McCawley, J. D. 1972, Syntactic and Logical Arguments for Semantic structures. Bloomington Ind.

Mugdan, J. 1984, Jan Baudouin de Courtenay (1845 - 1929)-Leben und Werk. München.

Paulsson, 0. 1974, Aspects of Polish Verb Morphology and Phonology. Göteborg.

Paulsson, 0. 1979, Asymilacja niektorych połaczen społgłoskowych w fonologii polskiej. In: Meddelanden frán institutionen för slaviska och baltiska sprâk - Stockholm 1979 Nr. 20 - Papers on Slavonic Linguistics 3, S. $39-51$.

Pohl, A. 1979, Teoria językowa Jana Baudouina de Courtenay z perspektywy generatywistyki. In: M. Szymczak (Hrsg.), Jan Baudouin de Courtenay a lingwistyka Swiatowa. Warszawa. (Angeblich im Druck)

Pohl, A. 1980a, Grundlegungen zur Morphonologie der polnischen Gegenwartssprache. In: Zeitschrift für Slavlsche Philologie XLI, Heft 2, S. $350-379$. 
Pohl, A. 1980b, Morphonologie des Diminutivs in der polnischen Gegenwartssprache. In: Scando-slavica 26, s. $161-173$.

Pohl, A. 1983a, Der Nominativus Pluralis der Nomina in der polnischen und in der russischen Gegenwartssprache. Ein Beitrag zur Morphonologie der Flexion in den slavischen Sprachen. In: R. Olesch (Hrsg.), Slavistische studien zum IX. Internationalen SlavistenkongreB in Kiev 1983, S. $361-386$.

Pohl, A. 1983b, Morphonologie des Genitivs im Russischen und im Polnischen der Gegenwart. In: G. Freidhof et al. (Hrsg.), Studia slavica in honorem viri doctissimi olexa Horbatsch. Teil II: Beiträge zur ostslavischen Philologie. München. S. $109-130$.

Pohl, A. 1983C, Der Genitivus in der $\overline{\mathrm{X}}$ - Syntax der polnischen und der russischen Gegenwartssprache. In: Scando-slavica 29. S. 177 - 190 .

Pohl, A. 1983d, Posesywnosé w gramatyce generatywnej współczesnego języka polskiego. In: Polonica IX, S. 29 - 36.

Pohl, A. 1984, Die Possessivität in der generativen Grammatik der russischen Gegenwartssprache. In: Zeitschrift für slavische Philologie 44, Heft 1, s. 193 - 207.

Radford, A. 1981, Transformational Syntax. A Student's Guide to Chomsky's Extended standard Theory. Cambridge et al.

Schmid, W. P. 1970, Skizze einer allgemeinen Theorie der Wortarten. In: Abhandlungen der Geistes- und Sozialwissenschaftlichen Klasse der Akademie der Wissenschaften und der Literatur. Jahrgang 1970. Nr. 5. Mainz - Wiesbaden. S. 257 - 280.

Szober, S. 11962, Gramatyka jezyka polskiego. Warszawa.

Weinreich, U. 1966, Explorations in Semantic Theory. In: T. A. Sebeok (Hrsg.) Current Trends in Linguistics 3. Theoretical Foundations. The Hague - Paris. S. $395-477$.

Wierzchowska, B. 1980, Fonetyka i fonologia języa polskiego. wrockaw et al.

Wurzel, W. U. 1970, Studien zur deutschen Lautstruktur. In: Studia Grammatica VIII. Berlin. 
INVESTIGATION IN WORD-FORMATION - THE PROBLEM OF NOMINALIZATION

IN MODERN STANDARD POLISH

\section{SUMMARY}

The description of word-formation in Polish demands:

- the incorporation of phonology, 1.e. morphonology

- the determination of inherent features that correlate with the rich affixation

- a morphosyntactic solution for the assignment of numerous affixes

- the formulation of affixation rules

- the investigation of representative data material.

Phonology must be taken into consideration for the description of word-formation because Polish has to be counted among the languages with a comparatively very high number of correlational alternations of both consonants and vowels, even among the Slavic language family. Consonantal alternations mark the boundaries of formatives and determine the realization of vocalic alternations in affixes and roots. In this respect, the rules of nominalization depend on a complex of phonological, 1.e. morphonological rules. These rules have to include elisions which among other things prevent $\underline{V} \underline{V}$-sequences. Furthermore, consonantal alternations not involved in word-formation, phonetically conditioned alternations, are formulated as rules as well.

On the level of morphonology, inner and outer word boundaries are set into a generalized word marker, thus defining the underlying morphosyntactic structure of Polish words. Morphosyntactic structure consists of two parts: the one of word-formation and an inflexional one. Both parts are determined by morpheme markers which are defined as to boundary, position, and function as prefix, root, infix, stem formative, stem marker, classifier, evaluator and flective. The classifier determines the lexical category - $N$ in our investigation - of the word to be formed, the stem marker on the other hand the lexical category of the word stem. The morphemically marked and morphonemically represented morphosyntactic structure makes up the $W$-base of the noun considered.

For the formation of nouns in Polish, besides the features of 
gender the following traditional criteria are necessary as well: COUNTABLE, ANIMATE, HUMAN, and AGENT. Additionally, some further features have to be included. Inherent features condition the assignment of affixes and the correspondence with certain nominal groups or paraphrases that underly all nominalized forms in Po11sh.

The category trace can be utilized in order to explain all potential or existing nominal groups and paraphrases in Polish differentiating between marked and unmarked components of the nominal group. Trace, following Chomsky 1981, is never lexicalized. Trace guarantees the assignment of the nominal classifier to the unmarked word stem. Nominal group and possibly corresponding noun, which both have to be formed, share the same inherent features. The unmarked component additionally takes over the inherent features of the marked component.

Underlying noun groups cannot be part of the S-base. The generalized w-marker takes over that place, thus determining the morphosyntactic structure and consequently the w-base. The generalized $W$-marker is inserted under the node marked by $N$.

Derivational and affixational rules are formulated for each nominal $W$-base. These derivational rules effect the nominalization of word stems, which are characterized by different lexical categories - not only main categories -, and they effect as well the nominalization of the root, being a necessary component of Polish word-formation. The affixational rules determine the morphophonemic representation of the nominal classifier. They are conditioned by the morphonemic representation of the stem marker and the inherent features.

The investigated data material was selected mainly with respect to productivity and markedness and can be regarded as representative as to these criteria. Markedness is of special importance for three genders, which are reflected in affixation.

For those instances of so-called "Univerbierung" that do not - from the synchronic point of view - reveal in the underlying nominal groups the conditions for this phenomenon, the marked part is replaced by features associated with the "univerbized" nouns. 
The intergration of the nominal group into word-formation does not mean that transformations are introduced into the structure of the W-base. Nominal group and noun to be formed can have the same inherent features, but will be derived by different wordformation rules. 
BADANIA NAD SEOWOTWORSTWEM - PROBLEM NOMINALIZACJI WE WSPOECZESNYM JEZYKU POLSKIM

\section{STRESZCZENIE}

Słowotwórstwo wspołłczesnego jezyka polskiego wymaga $\mathrm{m}$. in.:

- uwzględnienia fonologil, t.j. morfonologii,

- morfosyntaktycznego rozwiazania dla afiksacji,

- postulowania cech inherentnych zwiazzanych 2 bogata afiksacja,

- reguł derywacyjnych i afiksacyjnych.

- analizy reprezentatywnego zestawu słow.

Fonologia, t.j. morfonologia w słowotwórstwie języka polskiego jest m.in. dlatego nieodzowna, poniewai wśód języków słowianskich język polski posiada najwięssza ilose alternacji zarówno społłłoskowych jak 1 samogłoskowych, ktorych klasyfikacja oparta jest tu na klasyfikacji Baudouina de Courtenay. Alternacje spógłoskowe wyznaczaja granice formatywu 1 powoduja - 2 wyjatkiem tzw. e ruchomego - realizacje alternantów samogłoskowych zarowno w afiksach jak i w rdzeniu (Wurzel) słowa. Dlatego tei zestaw reguł nominalizacyjnych wklucza system reguł morfonologicznych, według ktorych przebiega realizacja alternantow korelacyjnych, t.j. alternantów 2 motywacja morfologiczna, oraz realizacja alternantow dywergencyjnych, $t . j$. alternant $\delta w z$ motywacja fonetyczna. W skład reguł morfonologicznych wchodza takie reguły zapobiegajace tworzeniu m.in. dyftongów.

W zasięgu morfonologii wyznaczone sa także zewnętrzne 1 wewnętrzne granice słowa, będace składnikami struktury morfosyntaktycznej. Struktura morfosyntaktyczna stanowi podłoże każdego słowa tworzonego w języku polskim. Składa się ona z dwóch częsci, z częsci słowotworczej 1 z cześci fleksyjnej. Obydwie te czesci składaja się z "markerów" morfemicznych (Morphem-Marker) zdefiniowanych względem granic, pozycji i funkcji jako prefiks, rdzen, infiks, formatyw tematowy (Stamm-Formativ), "marker" tematowy (Stamm-Marker), klasyfikator, ewaluator i flektyw. Klasyfikator okresla kategorie leksykalna, tutaj N, tworzonego słowa. "Marker" tematowy natomiast okresla kategorie leksykalna tematu (Wort-Stamm). Morfemicznie nacechowana $i$ morfonemicznie reprezentowana struktura morfosyntaktyczna tworzy baze słowa (W-Basis). 
Oprócz cech rodzaju gramatycznego w słowotworstwie rzeczowników polskich potrzebne sa kategorle tradycyjnej gramatyki jezyka polskiego, mianowicie: POLICZALNOSC, ZYWOTNOSC, OSOBOWOSC oraz AGENS. Kategorie te maja funkcje cech inherentnych przyporzadkowanych reprezentacj 1 morfonemicznej bazy słowa. Wobec bogactwa afiksów cechy te jednak sa niewystarczajace, dlatego tez musza byc uzupełnione przez dodatkowe. Cechy inherentne przyporzadkowane sa zarówno reprezentacji poszczegolnych "markerów" morfemicznych bazy rzeczownika tworzonego jak 1 grupom wielowyrazowym lub parafrazom majacym z rzeczownikiem tworzonym znaczenie wspolne.

w celu wcielenia moziliwych grup wielowyrazowych do słowotworstwa moina posłuzyć sie kategoria sladu (trace), aby odróninic składniki leksykalne nacechowane od nienacechowanych. Ponieważ kategoril Sladu według Chomsky'ego nie można zastapit słowem, odpowiada ona przyporzadkowaniu klasyfikatora nominalnego tematowi lub rdzenlowi nienacechowanemu tworzonego rzeczownika. Potencjalna grupa wielowyrazowa 1 odpowiadajacy jej znaczeniowo rzeczownik maja więc te same cechy inherentne. Cechy inherentne przyporzadkowane nacechowanym składnikom grupy wielowyrazowej sa identyczne $z$ cechami inherentnymi przyporzadkowanymi klasyfikatorowi tworzonego rzeczownika.

Ponlewaz grupy wielowyrazowej do struktury bazowej zdania wstawic nie mozina, przyjmuje sie ogolny "marker" słowa (generalisierter W-Marker) tworzacy strukture morfosyntaktyczna oraz baze słowa. Ogolny "marker" słowa, w skład którego wchodza leksykalna częsć formatywna oraz gramatyczna częsć formatywna, moze byc wstawiany pod węzłem nacechowanym przez $\mathrm{N}$.

Dla nominalnej bazy słowa postuluje sie reguły derywacyjne oraz reguły afiksacyjne. za pomoca reguł derywacyjnych przeprowadza sie nominalizacje tematów oznaczonych przez poszczególne - 1 to nie tylko głowne - kategorie leksykalne. 2 a pomoca regul derywacyjnych przeprowadza się takie nominalizacje rdzenia, z ktorej m.in. ze względu na słowa-zapożyczenta w jezyku polskim rezygnować nie można. Za pomoca reguł afiksacyjnych tworzy sie reprezentacje morfonemiczne klasyfikatora nominalnego. Reguły afiksacyjne uwzgledniaja takie reprezentacje morfonemiczne "markera" tematowego oraz cechy inherentne jako warunek afiksacji. 
Materiał słowny został wybrany zwłaszcza pod względem produktywności 1 relacji nacechowania vs. nienacechowania majacej szczegolnie w rozroinieniu trzech rodzajow gramatycznych wpływ na afiksacje.

Jesli na podstawie cech inherentnych wspolnych grupie wielowyrazowej 1 odpowiedniemu rzeczownikowi przyjmujemy tzw. "uniwerbizacje", wtedy możemy w wypadku braku odpowiedniej grupy wielowyrazowej zastapis jej składniki leksykalne odpowiednimi cechami inherentnymi. Lecz mimo wspolnych cech inherentnych, jakie moga miec zarówno rzeczownik jak i grupa wielowyrazowa, przedstawiona $w$ pracy niniejszej nominalizacja w jezyku polskim wyklucza transformacje. Potencjalna grupa wielowyrazowa 1 rzeczownik maja mimo wspolnego znaczenia odmienne reguły derywacyjne: swiadczy o tym nie tyle przedstawiona tumetoda opisu ile sam przedmiot, na ktorym metoda jest oparta.

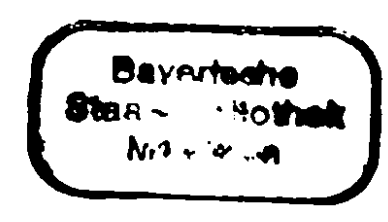

